\title{
Pre-clinical radiotherapy
}

Citation for published version (APA):

Vaniqui de Santana, A. C. (2019). Pre-clinical radiotherapy: from imaging to dose. [Doctoral Thesis, Maastricht University]. Gildeprint en Universitaire Pers Maastricht. https://doi.org/10.26481/dis.20191212av

Document status and date:

Published: 01/01/2019

DOI:

10.26481/dis.20191212av

Document Version:

Publisher's PDF, also known as Version of record

\section{Please check the document version of this publication:}

- A submitted manuscript is the version of the article upon submission and before peer-review. There can be important differences between the submitted version and the official published version of record.

People interested in the research are advised to contact the author for the final version of the publication, or visit the DOI to the publisher's website.

- The final author version and the galley proof are versions of the publication after peer review.

- The final published version features the final layout of the paper including the volume, issue and page numbers.

Link to publication

\footnotetext{
General rights rights.

- You may freely distribute the URL identifying the publication in the public portal. please follow below link for the End User Agreement:

www.umlib.nl/taverne-license

Take down policy

If you believe that this document breaches copyright please contact us at:

repository@maastrichtuniversity.nl

providing details and we will investigate your claim.
}

Copyright and moral rights for the publications made accessible in the public portal are retained by the authors and/or other copyright owners and it is a condition of accessing publications that users recognise and abide by the legal requirements associated with these

- Users may download and print one copy of any publication from the public portal for the purpose of private study or research.

- You may not further distribute the material or use it for any profit-making activity or commercial gain

If the publication is distributed under the terms of Article $25 \mathrm{fa}$ of the Dutch Copyright Act, indicated by the "Taverne" license above, 


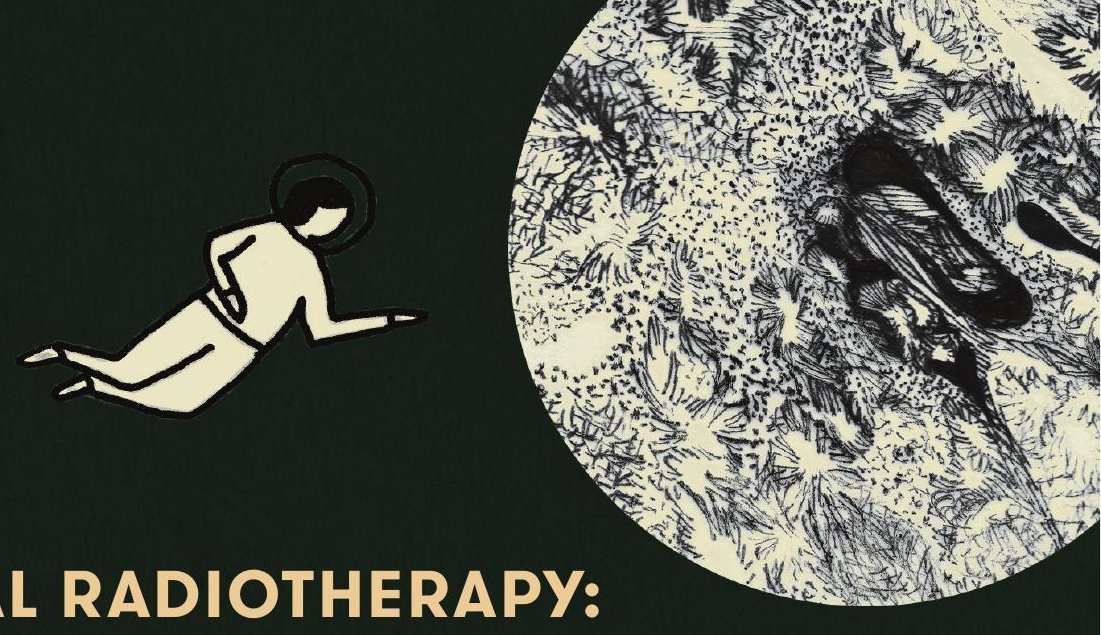

from imaging to dose

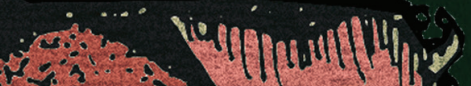



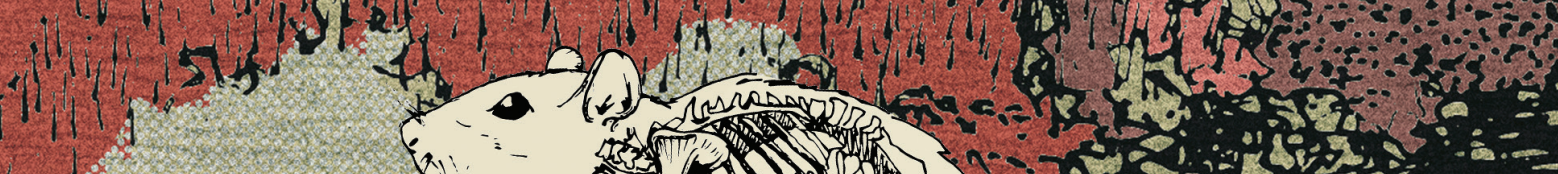

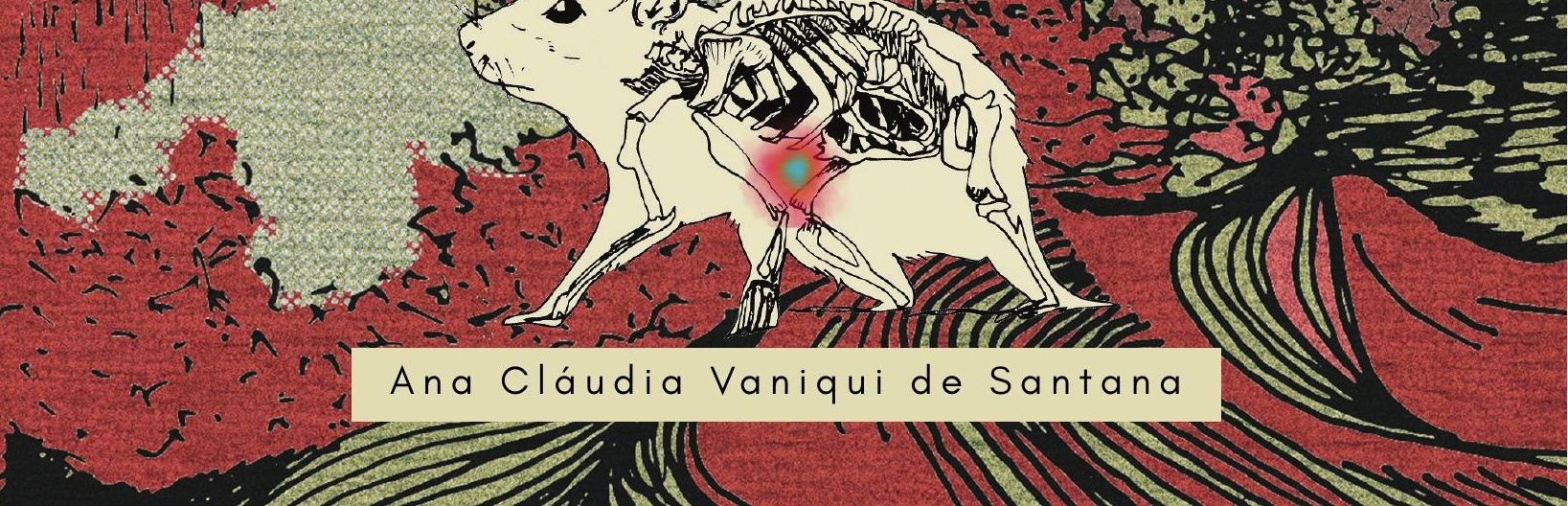





\section{PRE-CLINICAL RADIOTHERAPY: from imaging to dose}

Ana Cláudia Vaniqui de Santana 


\title{
Production
}

Cover

THE HUMAN EXPEDITION INTO PRE-CLINICAL SCIENCE by Laura Vaniqui

(C) Copyrights

copyright Ana Vaniqui, Maastricht 20 I 9

Printing: Gildeprint - Enschede

ISBN: 978-94-6323-964-6

\begin{abstract}
All rights reserved. No part of the material protected by this copyright notice may be reproduced or utilized in any form or by any means, electronic or mechanical, including photocopying, recording or by any information storage and retrieval system, without written permission from the author and the publisher(s) holding the copyrights of the reprinted article(s).
\end{abstract}




\title{
Pre-clinical radiotherapy: from imaging to dose
}

\author{
dissertation by:
}

Ana Cláudia Vaniqui de Santana

To obtain a doctoral degree at Maastricht University, on the authority of the Rector Magnificus Prof. dr. Rianne M. Letschert, in accordance with the decision of the Board of Deans, to be defended in public on Thursday, I2th of December 2019 at I6:00 

à minha querida mãe to my beloved mother 


\section{Supervisor}

Prof. Dr. Frank Verhaegen

\section{Co-Supervisor}

Dr. Gabriel Paiva Fonseca

Dr. Ludwig Dubois

\section{Assessment Committee}

Prof. Dr. Marc Vooijs (chairman)

Dr. Mark Hill, University of Oxford, United Kingdom

Dr. Marijke De Saint-Hubert, SCK•CEN, Belgium

Prof. Dr. Dirk K. M. De Ruysscher

The work presented in this thesis was made possible by the financial support of CAPES Scholarship and Science without Borders program from Brazil (BEX I2030/13-9). 

C
0

Chapter 2 The impact of dual energy CT imaging on dose calculations for pre-clinical studies

Chapter 3 The effect of different image reconstruction techniques on pre-clinical quantitative imaging and dual-energy CT

Chapter 4 On the determination of planning target margins due to motion for mice lung tumours using a four-dimensional MOBY phantom

Chapter $\mathbf{5}$ Cavity theory applications on pre-clinical irradiation

Chapter $\mathbf{6}$ Exploring the feasibility of a clinical proton beam with an adaptive aperture for pre-clinical research

Chapter 7 Summary, discussion, and prospective work

\section{Appendices}

List of abbreviations

Societal impact and valorisation

Acknowledgements

Curriculum vitae 



\section{INTRODUCTION}

introduction and outline 


\section{Introduction}

\section{I.I Cancer treatment: panorama}

The oldest acknowledged description of cancer, an Egyptian papyrus, believed to reference breast cancer and other tumours or ulcers, dates back to the zoth century BC. It describes suturing by cauterization with a "fire drill" and warns that there is no successful treatment for protruding tumours spread over the breast ${ }^{[\mathrm{I}]}$. The word cancer itself appears much later in history, introduced by Hippocrates $(460-370$ BC) as the Greek term karkinos (and karkinomas) and later translated to Latin into cancer. The term is an allusion to crab, as swollen blood vessels around breast cancer might look like crab legs ${ }^{[2]}$, thus carcinoids (or crab-like). Currently, cancer is the second leading cause of death worldwide, in 20I8, it was responsible for approximately 9.6 million deaths, its economic cost was estimated at US\$ I.I6 trillion and it is predicted that by 204029.5 million people will be living with it ${ }^{[3]}$. Cancer is also a generic term for a collection of diseases (there are over 200 cancer types) involving unregulated cell growth and characterized by underlying processes, which discriminate healthy from cancerous cells $s^{[4,5]}$. The processes or the hallmarks of cancer are summarized in Figure I.I; cells need to have acquired mutations in all hallmarks before they can be seen as cancerous, for which the order is not set: different mutations paths can lead to cancer.

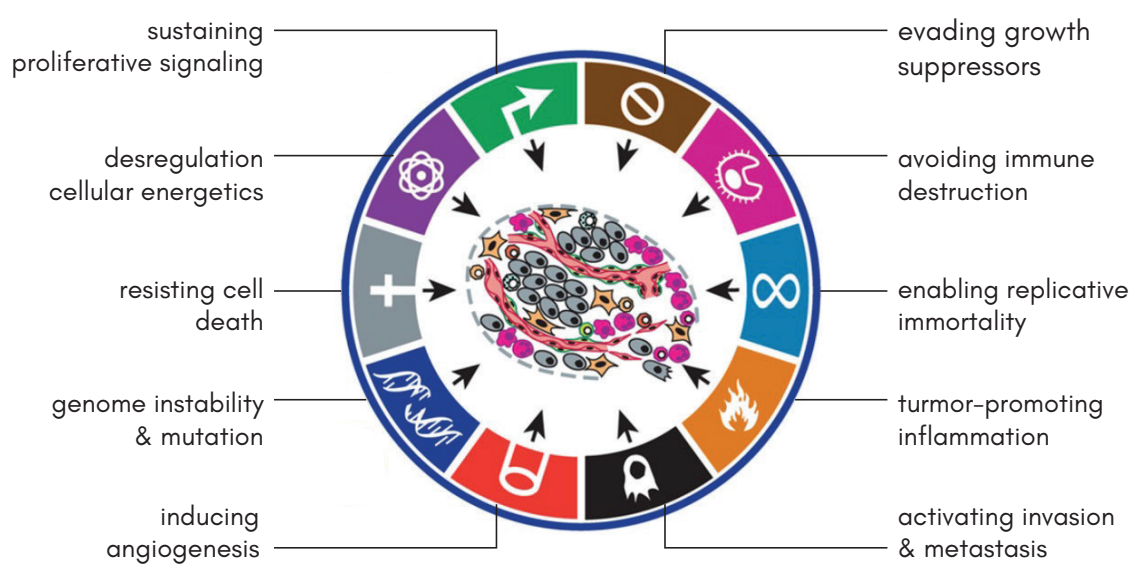

Figure I.I - The biological capabilities of human tumours, the hallmarks of cancer, comprehend: sustaining proliferative signalling, evading growth suppressors, enabling replicative immortality, activating invasion and metastasis, inducing angiogenesis, resisting cell death, reprogramming of energy metabolism and evading immune destruction. This is in addition to underlying genome instability and inflammation. Besides cancer cells, tumours contain normal cells that create the tumour microenvironment. Reproduced and modified with kind permission. Hanahan D, Weinberg RA. Cell 20I1 ${ }^{|5|}$. 
Cancer treatment has evolved with variable success over the years. Surgery, chemotherapy and radiotherapy are the most common treatment modalities, which also comprehend hormonal therapy, immunotherapy, gene therapy and targeted treatments. Surgery, the physical resection of neoplasia, was already practiced in the eighteenth century for early-stage tumours ${ }^{[6]}$. Techniques were developed under standard oncological criteria during the twentieth century ${ }^{[t]}$ and nowadays benefit from modern technology, e.g. non-invasive procedures. Chemotherapy, a sub-product of research on chemical weapons during the World War $\mathrm{II}^{[7]}$, targets rapidly dividing cells and blocks different functions in cell growth and replication using systemic drugs. Modern chemotherapy avails from novel drug combinations, delivery techniques and targeted approaches, such as monoclonal antibody therapy ${ }^{[8]}$.

Radiotherapy, the main focus of this thesis, uses ionizing radiation with a curative intent, targeting cancerous tissues and preserving healthy ones to the maximum possible extent. It has been heavily practiced over the last century. Only seven months after the discovery of $\mathrm{x}$-rays by Röntgen, in July 1896 a gastric carcinoma radiation therapy was performed ${ }^{[9, \text { rol] }}$. By I90I, also shortly after the discovery of spontaneous radioactivity by Becquerel and the Curie couple, patients in Paris and New York (1904) were undergoing implantation of radium tubes directly into tumours, some of the first interstitial radiotherapy, or brachytherapy, treatments ${ }^{[\mathrm{It}, \mathrm{rl}}$. The first light ion therapy treatment took place in 1954 for pituitary adenomas using protons and subsequently helium ion beams ${ }^{\left[{ }^{[r]}\right]}$. Throughout the twentieth century external beam radiation therapy (EBRT) evolved drastically: it passed from superficial and orthovoltage photons in the kilovolt $(\mathrm{kV})$ energy range produced by $\mathrm{x}$-ray tubes and sealed radioactive sources to megavoltage (MV) ${ }^{60} \mathrm{Co}$ machines, medical linear accelerators, electron and heavy particle beams ${ }^{[\mathrm{r} 2}$. Similarly, the use of large radiation fields, which deposited radiation dose beyond the target and restricted the tumour control probability (TCP) by limiting the normal tissue complication probability (NTCP) gradually transitioned to spatio-temporal modulated and highly conformal techniques in the last twenty years ${ }^{\left[{ }^{[14}\right]}$. Newer devices are able to deliver multi-field four-dimensional treatment plans, and arrest deeper and spread tumours while promoting higher sparing of healthy structures. 
On a parallel track, the understanding of oncogenesis and response of biological matter to ionizing radiation followed a similar revolution, albeit moderately delayed with respect to patient therapy. In I906, radiosensitivity was detected in cells with high mitotic activities and low levels of differentiation ${ }^{[5]}$. Two decades later, Muller showed that chromosomes are the main target in ionizing radiation cell killing ${ }^{[16,17]}$. The principle of fractionation was first demonstrated in I9II, as distributing radiation dose in multiple therapy sessions lead to skin burn (erythema) avoidance $^{[\mathrm{r} 8]}$. Fractionation regimens remained inconsistent until several decades later, when a better understanding of time-dose relationships and the differential recuperation characteristic of normal and neoplastic tissues led to the conclusion that independent processes occur between fractions and favour the survival of normal over cancer tissues ${ }^{[19]}$. Yet, they are still not standardized. Additionally, since the i920's, oxygen has been linked to cellular response to radiation: the currently accepted mechanism considers that it acts in free radical-induced DNA damage repair and recent research indicates that tumour hypoxia can generate a broad range of signalling effects $^{[12,20,21]}$.

In addition to radiotherapy and radiobiology advances, the development of diagnostic imaging has been a fundamental tool for the evolution of cancer care. The advent of the computed tomography (CT) and its integration to radiotherapy allowed anatomical visualization of targeted and normal structures. It became a platform upon which three-dimensional (3D) radiation dose calculations are performed and dose distribution throughout the entire irradiated volume is quantified. The use of CT in treatment planning resulted in greater precision in dose distribution, optimization and patient and dose positioning, decreasing the risk of accidental target misalignment and related complications. In addition to anatomical and structural imaging, the ability to spatially measure biological, metabolic, or physiological processes was achieved with functional imaging modalities such as magnetic resonance imaging (MRI), single photon emission tomography (SPECT) and positron emission tomography (PET). On a biological standpoint, enhanced microscopy and non-invasive functional imaging with metabolic tracers enabled insight in microenvironment repair/response pathways and the targeting of e.g. hypoxia.

Modern external beam photon radiotherapy is delivered with highly conformal beams in short treatment times, where small tumour subvolumes can accurately receive high doses. It benefits from adaptive methods 
that combine image-guided techniques capable of optimizing treatment during its course $e^{\left[{ }_{4}\right]}$. Moreover, external beam light ion therapy is globally widespread, with 91 centres in operation in $2019^{[22]}$, characterized by the major energy deposition at a specific depth within the patient and, unlike photon treatments, the preservation of normal tissues beyond that point. These therapies can be used in an adjuvant fashion, whereby a combination with surgery or chemotherapy may optimize treatment outcome. Biological variables based on differences between tumour metabolism, tumour antigens, and normal tissues have also been incorporated into the treatment process contributing to individualized precise medicine.

The brief contextualization above, from primitive to state-of-the-art treatments, the parallel development of physical and biological principia and the technological revolution, portrays the evolution of cancer care in time and the intrinsic multidisciplinary characteristics of this field. Figure I.2 gives an abridged timeline of main radiotherapy events. Although much has changed since the early $2 \mathrm{O}^{\text {th }}$ century, when an $\mathrm{x}$-ray image could take hours ${ }^{[23]}$ and only small localized tumours were curable, the urgent need to treat patients remained invariable: it is the force that drives the empirical aspect of this field. Historically, treatment regimens for site-specific cancers have not been based on randomized clinical trials, fundamental biological models or epidemiological studies. Even today, the shift from non-conformal large beams to sophisticated conformal irradiation beams has not been thoroughly validated on animal models or human trials. If conformal beams provide good target coverage, tumour cells might still exist outside the irradiated volume and physiological motion can affect the conformity: there is always a balance. However, the compromise between cure and toxicity has been settled in treatment protocols, based on consensus opinions on the best trade-off for a specific patient population ${ }^{[24]}$, often derived from large field, single-beam irradiations ${ }^{[25]}$, unspecific for current therapies.

Rigorous radiation research and multi-centre trials have brought benefits in terms of understanding outcome in modern care ${ }^{[27]}$. Pre-clinical research platforms can provide a cost-effective experimental pipeline for timely validation of new treatment modalities or interpretation of clinical experience on large cohorts ${ }^{[28]}$. In several fields, it is not an incipient practice; animal models and cell culture experiments are heavily used to evaluate new pharmaceuticals and systemic drugs due to legal requirements and controlled experimental conditions ${ }^{[29,30]}$. Pre-clinical nuclear medicine 


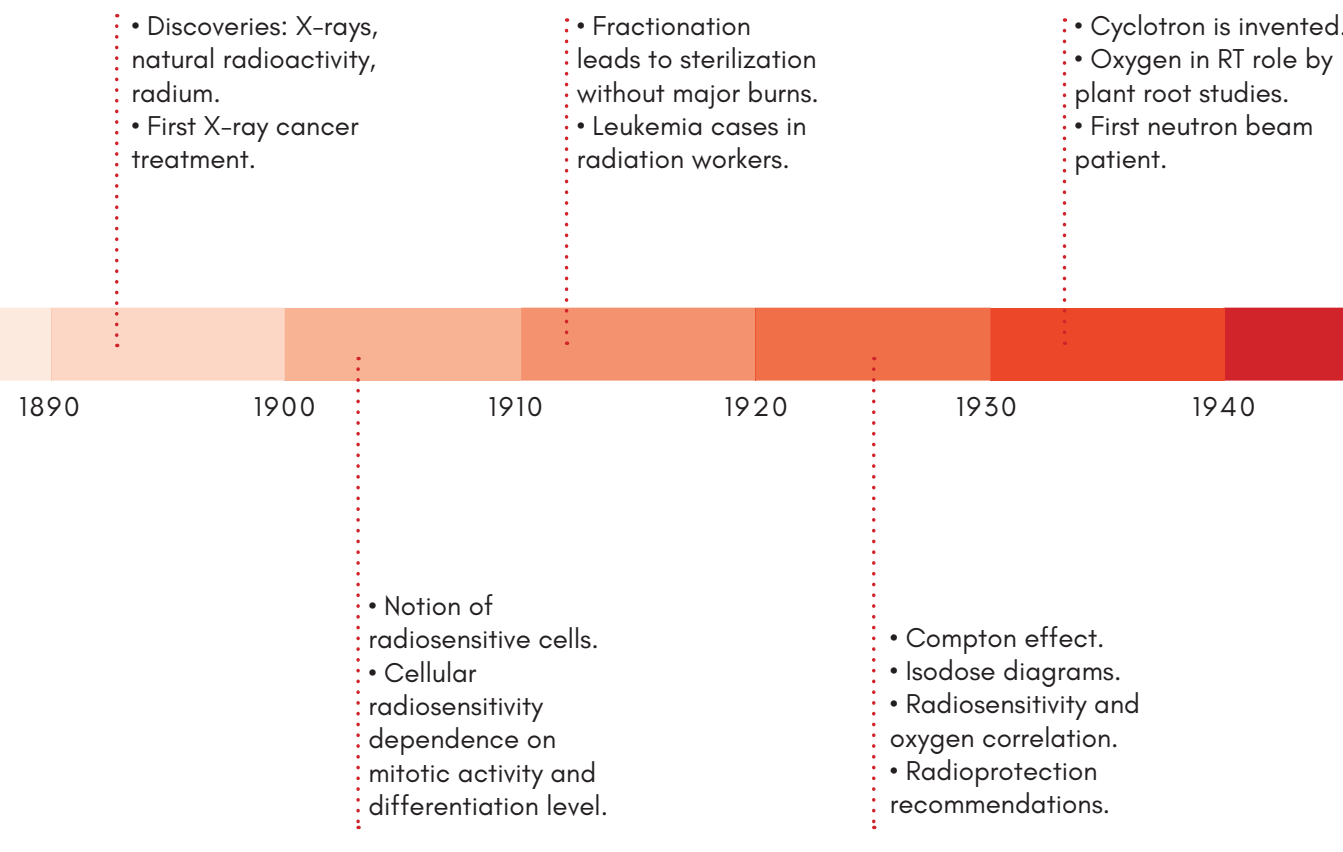

Figure I.2 - Abridged timeline: clinical, biological and physical advances of radiotherapy throughout the $20^{\text {th }}$ century. Adapted from Connel et $\mathrm{al}^{[\mathrm{I2}]}$ and Bernier et $\mathrm{al}^{[26]}$

research heavily relies on high-resolution functional small animal imaging to assess new radio-pharmaceutical tracers or therapies ${ }^{\left[3{ }^{3}\right]}$. EBRT on the other hand, with its vast number of daily treatments and different modalities, has had marginal contributions from small animal research ${ }^{\left[3_{2}\right]}$. To unravel fundamental questions of radiotherapy regarding ideal dose distributions, fractionation schemes, heterogeneous irradiations, metastatic pathways, combinations of radiation and other therapeutic or protective agents it is envisioned that pre-clinical precision radiotherapy, which realistically mimics clinical radiotherapy downscaled to small animal sizes, should play a larger role.

\section{I.2 Pre-clinical radiotherapy research: the paradigm shift}

Small animals are used to evaluate therapeutic profiling at low cost and maintenance in rapidly reproducing models $s^{[33]}$. They enable the investigation of treatment modalities that would be considered unethical in patients. Advances in clinical treatment capabilities and deeper understanding of 
- ${ }^{60}$ Co telegraphy.

- Oxygen effect quantification.

- First proton patient.

- Clonogenic survival curves for

irradiated cells.

- Cellular radiation damage

repair shown.
- First CT scans.

- PET developed.

- First hypoxic cell

sensitizer.

- Concept of IMRT.
- Cancer cell survival correlated with TCP.

- Bystander effect.

- ATM gene discovered.

- Functional imaging, dose painting, dose sculpting.
1950

1960
1970

- Differential

radiosensitivity.

- Hyperbaric oxygen in RT.

- First in vivo radiation

survival curve.

- Gamma knife for cranial radiosurgery.
1980
1990

2000

- Metastasis may occur before detection of primary tumours; differential radiosensitivity of early vs late responding tissues; multi-leaf collimator; MRI clinically available; first trials of chemo-radiation; clinical proton therapy; iso-effect formula based on quadratic and linear components of radiation-induced cell kill; tumour potential doubling time; development of IMRT.

radiation responses of normal and tumorous tissues can be achieved through pre-clinical trials. Similar to clinical care, pre-clinical radiotherapy has begun with primitive setups, large radiation fields and no imaging capabilities. Clinical devices have widely been used for small animal irradiation, although they often have higher energies and lower precision than ideal for the small specimens' dimensions. Dose has been rarely measured or estimated due to the lack of treatment planning systems ${ }^{\left[{ }^{22}\right]}$. Additionally, tumour models have typically been derived from xenograft transplantation of human (or cell culture) tumour tissues placed subcutaneously in the animal flank, which might fail to replicate the tumour micro-environment and to represent clinical response or tumour progression ${ }^{[34,35]}$. The poor conformity and resemblance to clinical treatments has possibly been an underlying cause for the limited pre-clinical radiotherapy data available and translation to clinical parameters. A number of research institutions and commercial companies have addressed the shortcomings of pre-clinical radiotherapy systems $s^{[32,36-40]}$ and proposed solutions: a few of them will be shortly mentioned on the following sections. In addition, Table i.I lists the 


\begin{tabular}{|c|c|c|c|c|}
\hline \multicolumn{4}{|c|}{ Ideal requirements for a small animal image-guided irradiator device } & \multirow{4}{*}{$\begin{array}{l}\text { Notes } \\
\text { X-ray tube potential } \\
\text { Based on Io min for } 2 \text { Gy } \\
\text { skin dose }\end{array}$} \\
\hline Beam energy & & IOO-300 kVp & & \\
\hline \multirow[t]{2}{*}{ Min dose rate } & \multicolumn{3}{|c|}{0.2 Gy $\min ^{-1}$} & \\
\hline & Rabbit & Rat & Mouse & \\
\hline $\begin{array}{l}\text { Image } \\
\text { Resolution }\end{array}$ & $0.33^{-0.66 \mathrm{~mm}}$ & $0.15-0.31 \mathrm{~mm}$ & $0.065-0.13 \mathrm{~mm}$ & $\begin{array}{l}\text { Desired isotropic voxel } \\
\text { size relative to voxel size } \\
\text { of } \mathrm{I}-2 \mathrm{~mm} \text { for patients }\end{array}$ \\
\hline $\begin{array}{l}\text { Image } \\
\text { temporal } \\
\text { resolution }\end{array}$ & I2O ms or $8 \mathrm{fps}$ & $75 \mathrm{~ms}$ or $\mathrm{I} 3 \mathrm{fps}$ & $40 \mathrm{~ms}$ or $25 \mathrm{fps}$ & $\begin{array}{l}\text { Based on one tenth of } \\
\text { respiratory period of free } \\
\text { breathing animals }\end{array}$ \\
\hline Beam diameter & I. $6-3.3 \mathrm{~mm}$ & $0.76-1.53 \mathrm{~mm}$ & $0.33-0.66 \mathrm{~mm}$ & $\begin{array}{l}\text { Desired beam size } \\
\text { diameter relative to } \\
\text { smallest pertinent clinical } \\
\text { beam diameter of } 5^{-10} \\
\text { mm for patients }\end{array}$ \\
\hline $\begin{array}{l}\text { Targeting } \\
\text { accuracy }\end{array}$ & $\pm 0.3 \mathrm{~mm}$ & $\pm 0.2 \mathrm{~mm}$ & \pm O.I mm & $\begin{array}{l}\text { The ideal targeting } \\
\text { accuracy will have } \\
\text { zero mechanical beam } \\
\text { positional error and be } \\
\text { limited only by image } \\
\text { resolution }\end{array}$ \\
\hline
\end{tabular}

Table I. I - Ideal requirements for an image-guided small animal irradiator device. Reproduced from Verhaegen et al, 20 I I ${ }^{[32]}$, with kind permission.

main requirements for small animal irradiation devices.

\section{I.2. I Irradiation energies}

Small animal external beam photon irradiations are currently performed in the $\mathrm{kV}$ (up to $300 \mathrm{kV}$ ) instead of the MV energy range to avoid large dose buildup (and re-buildup) regions near medium interfaces due to electronic disequilibrium and large beam penumbras ${ }^{[36]}$. Compton and photoelectric effects are the main physical interactions, which generate secondary particles responsible for dose deposition. The increased probability of photoelectric interactions distinguishes $\mathrm{kV}$ and MV therapy modalities. MV dose calculations may consider the irradiated volume, the patient's 
body, fully made of water for simpler absorbed dose calculations due to the predominance of Compton interactions ${ }^{[4]}$ which scale with the electron density of the medium. Incorrect media assignment for $\mathrm{kV}$ irradiations may lead to dose errors as a result of differences in energy absorption in different media and the increased probability of photoelectric effect: its cross section increases with increasing atomic number and decreasing photon energy ${ }^{[42-44]}$. Thus, energies above $100 \mathrm{keV}$ are preferred to avoid heterogeneous dose distributions which would not correspond to treatments with $\mathrm{MeV}$ beams, and could invalidate pre-clinical translation ${ }^{[45]}$. Exceptions to this practice can be differential tissue absorption or relative biological effectiveness (RBE) studies, as radiation effects can be boosted at lower energies ${ }^{[46]}$.

\section{I.2.2 Image guidance}

Similar to the clinical practice, CT imaging became the preferred modality for simultaneous anatomical information and treatment planning dose calculation for pre-clinical radiotherapy. Several other non-invasive imaging techniques exist for small animals, including MRI, SPECT, PET, optical imaging and ultrasound, which provide variable degrees of structural or functional information but cannot be used alone for dose calculations ${ }^{[47]}$. Nevertheless, they can be fused with CT scans for better contrast of soft tissue or enhanced tumour visualization accuracy. Commercial radiotherapy platforms use cone-beam CT (СВCT), which features an imaging panel and a dual-focus $x$-ray tube mounted in-line: the $\mathrm{x}$-ray tube is responsible for both irradiation and imaging in the same coordinate system, and the flat panel provides the sub-millimetric resolution (up to Iоo $\mu \mathrm{m}$ ) necessary for the tiny creatures ${ }^{[37,48]}$. Micro-CT devices with increased resolution $(<$ $20 \mu \mathrm{m})$ exist based on fan-beam technology. They are not integrated with current irradiation devices. The substantially increased spatial resolution necessary for pre-clinical imaging, one order of magnitude higher in comparison to the clinic, is fundamental for accurate and reproducible radiation guidance to match delivered with prescribed dose and to observe changes in tumorous and healthy tissues exposed to different radiotherapy fractionation schemes and techniques. In this scenario image quality is paramount.

In terms of hardware, $\mathrm{x}$-ray tubes with several possible potentials enable improved contrast-to-noise ratio and allow for dual-energy CT (DECT) capabilities; x-ray detectors with flat quantum efficiency, dose response and high temporal resolution facilitate dose-reconstruction and respiratory 
gating techniques ${ }^{[47,49,50]}$. Flat panel detectors require a number of calibrations prior to image acquisition and are supposed to be calibrated on a routine basis to ensure the accuracy, stability, and reliability of CT numbers ${ }^{[48,51]}$. The image projections have to be corrected for dark currents, differences in pixel gain, defective pixels and mechanical flex of the gantry. Figure I.3 shows a commercial small animal irradiation platform. Regarding software, analytical filtered back-projection algorithms are commonly used, while iterative reconstruction techniques are mostly used for fan-beam CT but can be implemented for the CBCT ${ }^{\left[5_{2}\right]}$. They are expected to yield improved image quality as they use multiple repetitions in which a current solution converges towards a better solution: image and projection data correction are repeated until a condition predefined by the algorithm is satisfied and the final image is generated $\left.{ }^{[53}, 54\right]$. Additionally, the usage of scatter and noise correction kernels is also desirable as CBCT is inherently a noisier technique and image artefacts may influence the accuracy of posterior dose calculations.

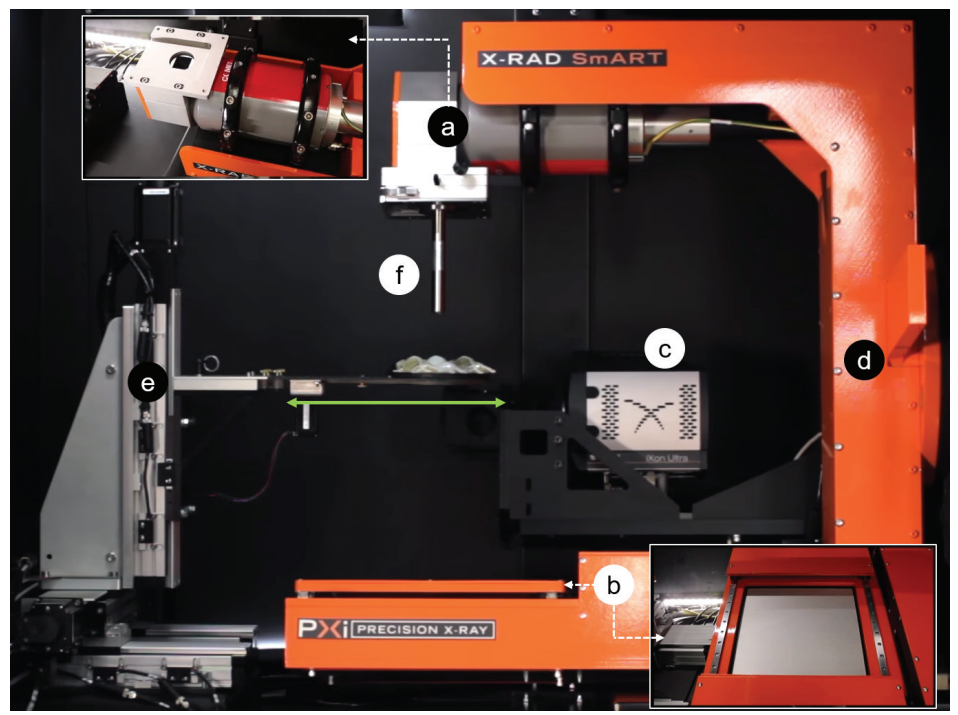

Figure I.3 - Commercial small animal irradiation platform: X-RAD SmART (Precision X-Ray, North Branford (CT), United States). (a) Dual focus x-ray tube used for imaging and irradiation. (b) Flat panel detector for the CT imaging system. (c) Bioluminescence camera. (d) Gantry of the machine which rotates around the subject. (e) Stage to position the specimens with six degrees of freedom: it moves forward or backward, left or right, and up or down, The green arrow indicates where the specimens are placed). (f) Collimator attached to the $x$-ray tube for irradiation purposes. Reproduced with permission from the website pxinc.com/resources/\#videos accessed on April 2019. 


\section{I.2.3 Treatment Planning}

In commercial pre-clinical systems, treatment planning follows similar steps to their clinical analogues. Subsequent to the CT scan acquisition, image voxels are assigned tissue density and type, taken at a specific radiation quality. The assignment is typically derived from a piecewise linear calibration curve, which correlates CT numbers to mass densities, using phantoms with reference materials. Subdivisions of the calibration curve into intervals that belong to a same medium assign the tissue type. In most cases only three materials are used: air, soft tissue and bone. This is an important step, as erroneous assignment is likely to be propagated into incorrect dose calculations, sub-optimal results and invalid conclusions ${ }^{\mathrm{I} 5}$. ${ }^{56}$. It has been hypothesized that DECT imaging may yield improved tissue segmentation as it has the advantage of extracting an additional material characteristic besides density ${ }^{[54,55,57-59]}$. This technique can be achieved with different $x$-ray spectra, different filtration to split a spectrum into two or by dividing the photons of a single spectrum into different energy bins at the detector level. Pre-clinically, it has been used with two consecutive scans and besides density, atomic number information was derived ${ }^{[58]}$. It has been shown that information on effective atomic number is needed to achieve accurate dose calculations and using a higher number of tissues, especially bones, might be advantageous ${ }^{[5,60]}$. Effects of single and DECT modalities on tissue segmentation and dose calculations, as well as image quality parameters, are studied in detail in Chapters 2 and 3.

\section{I.2.4 Dose calculations}

Monte Carlo (MC) techniques are considered the gold-standard to dose calculations due their detailed models of particle transport, interactions and production of secondary particles based on cross sections and transport theories. In principle, MC simulation is the only suitable technique for accurate $\mathrm{kV}$ photon dose calculations ${ }^{[6]}$. Small animal treatment dose calculations are currently performed with MC methods and other techniques based on ray-tracing or superposition convolution ${ }^{[25,62]}$. The latter methods are considered less accurate in non-water geometries and in cases where photon scatter is relevant. Commercial pre-clinical treatment planning systems exist based on MC calculations ${ }^{[25,63]}$. A complete example, Figure I.4, follows the workflow of a simplified clinical TPS: the animal image is uploaded, processed for density and material assignment, structures (e.g. tumours and organs at risk, OARs) are delineated, radiation beams 
defined, dose calculation is performed with a MC engine and dose analysis is displayed as dose volume histograms (DVHs) of the contoured volumes and their respective cumulative dose. If the treatment was successfully planned, a radiation device control file can be written for desired physical positioning and irradiation ${ }^{[63]}$. The radiation beams are generally shaped with fixed or variable collimators and generate small beams: between 0.5 $\mathrm{mm}$ and a few centimetres. Figure r. 5 shows different irradiation scenarios.

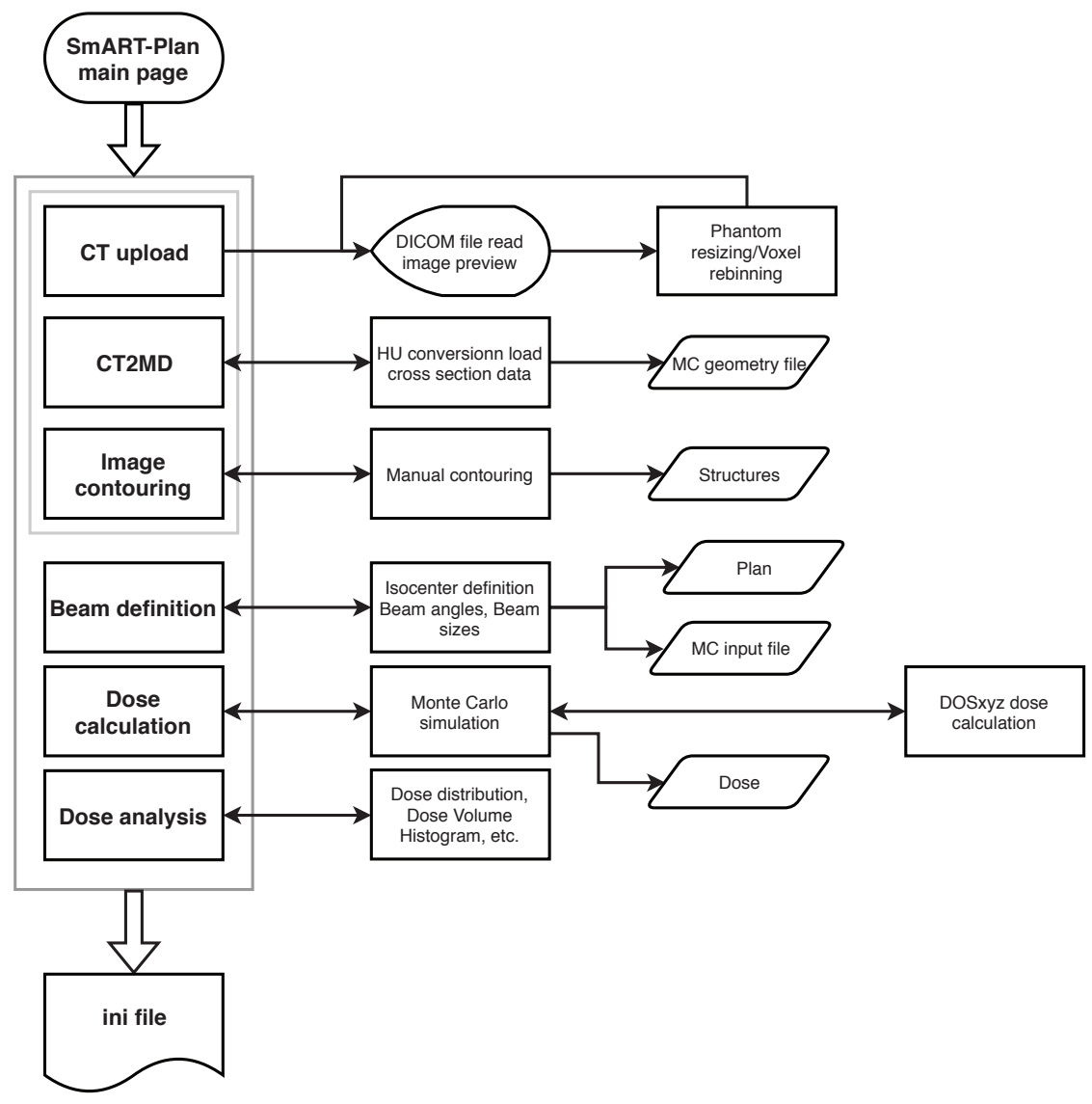

Figure I.4 - Example of commercial TPS workflow: the tasks on the left-hand side are performed sequentially top/down, from CT upload to Dose analysis. Reproduced from van Hoof et al, 2013 ${ }^{[63]}$, with kind permission. 

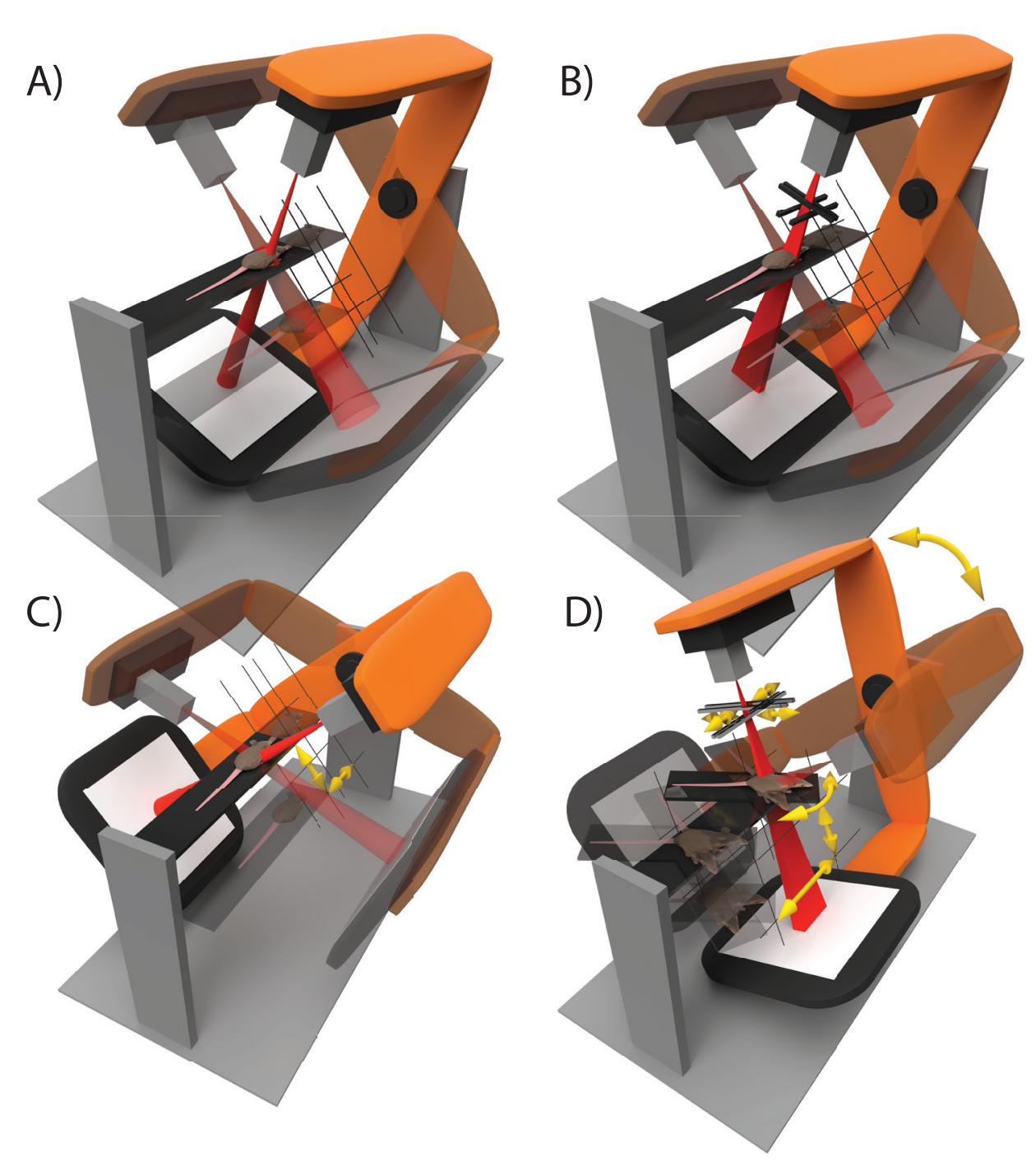

Figure I.5 - Irradiation scenarios: 3D translation of the animal and gantry rotation (a) with interchangeable collimators; (b) with variable aperture collimators. (c) Animal translation with a fixed beam size. (d) Dynamic collimator aperture, gantry angle, animal couch position and angle. Semitransparent elements indicate different states of static step and-shoot irradiations and yellow arrows indicate movement of the animal couch and beam collimators during radiation delivery. Reproduced from van Hoof et al, 2019 ${ }^{[64]}$, with kind permission. 


\section{I.2.5 Structural delineation}

The structural delineation, briefly mentioned above, is the process where an expert in the specimen anatomy uses a set of tools to precisely contour the tumour and organs of interest. It is a fundamental step in treatment planning as further dose analysis, and defines if the tumour dose coverage and simultaneous health tissue sparing are within the desired protocols. The image drawn upon is a static representation of the instants when the scan was taken. It should be considered that although the animal was physically restrained, under sedation, basal functions, such as breathing and heart motion, can promote a shift in the expected tumour positioning. In clinical radiotherapy, the use of target volumes and treatment margins have long been recommended and is a premise for unambiguous prescription, recording and reporting of dose ${ }^{[65-67]}$. Besides the delineation of the tumour itself, also called gross target volume (GTV), the expert contours a volume where malignancy spread is expected, clinical target volume (CTV). Both GTV and CTV are purely based on anatomical and biological parameters. An extra contour, the planning target volume (PTV), consists of a geometrical margin to account for variation in size, shape and position relative to the treatment beam. The PTV considers the net effect of all the possible geometrical variations, to ensure that the prescribed dose is absorbed in the CTV. A number of clinical studies have explored this concept and proposed recipes to quantify the weight of systematic and random errors, or the effect of treatment execution and preparation, on the size of the $\mathrm{PTV}^{[68]}$. The concept of margins is still incipient in small animal radiotherapy ${ }^{[69,70]}$. Although some studies use irradiation margins, there are no specific guidelines on how to define them ${ }^{[7]}$. Chapter 4 approaches this subject and proposes a framework for margin delineation. 


\section{I.2.6 Dose reporting}

Proper dose reporting is as important as correct tissue assignment and still being debated in the clinic $^{[72]}$. Monte Carlo dose engines require tissue identification and typically report the absorbed dose in terms of dose to medium-in-medium $\left(D_{\mathrm{mm}}\right)$, whereby particles are transported and dose is scored in the medium that was previously assigned to a specific voxel. Thus, material specific interaction coefficients are used. Some algorithms may use a different quantity: dose to water-in-medium $D_{\mathrm{wm}}$. In this case, particles are transported in the specific medium but dose is scored using water cross sections. The two absorbed doses are different quantities with large differences in the $\mathrm{kV}$ energy range and demand a conversion factor for proper comparison, which adds uncertainty. The factor derivation has a degree of complexity as it depends on the scoring volume and can be a combination of mass stopping power and mass-energy absorption coefficient, both ratios for water and medium ${ }^{[73,74]}$. The coexistence of both quantities is related to historically simpler dose calculation methods, the water-based aspect of radiation dosimetry and the ignorance regarding which quantity correlates best with biological effects of radiation. Chapter 5 treats this theme, with a thorough theoretical section and calculations in different scenarios that amplify the differences between the quantities.

\section{I.3 Light ion treatments}

Pre-clinical research is necessary to also advance the knowledge on ion beam therapies. This treatment modality is a reality in a rapidly growing number of centres worldwide ${ }^{[22]}$. Integrating the existing state-of-the-art pre-clinical photon expertise with ion beams is likely to initiate a novel field in small animal research. A few studies have been published on the feasibility of ion beam irradiations and the impact on tumour growth, survival and RBE in fractionated schemes ${ }^{[75-78]}$. The high cost associated with an ion beam facility hampers the possibility of dedicated small animal ion beam centres. However, clinical and non-clinical ion beam facilities have been investigating ways to adapt their setups for image guided pre-clinical research, using existing $x$-ray platforms for imaging and positioning. The use of a clinical proton beam, with the potential to be shaped into small fields with sharp beam penumbras and low proton energies might be attractive in this context. Chapter 6 explores dose calculations with a clinical proton beam. 


\section{I.4 Objectives}

Pre-clinical radiotherapy has evolved and matured into a platform for evaluation of new treatment modalities. A number of challenges to achieving downscaled treatments in small animals persist. In particular, the role of image quality and its translation into dose calculations, target delivery, dose reporting and ion beam feasibility form the basis of this thesis.

\section{I.5 Thesis outline}

Chapter I, gives an overview on the background and importance of radiation therapy, its historical evolution, empirical aspects and the rise and evolution of pre-clinical radiation research. For the latter, the need to replicate clinical scenarios is emphasized to achieve translational research.

Chapter 2 presents an investigation on the feasibility of using dual-energy CT (DECT) for tissue segmentation and $\mathrm{kV}$ dose calculations in pre-clinical studies and assesses potential dose calculation accuracy gain. Phantoms and an ex-vivo mouse, as well as, SECT and DECT segmentation techniques are compared and dose accuracy is evaluated.

Chapter 3 describes a software platform for pre-clinical CBCT $\mathrm{x}$-ray image reconstruction built using the open-source reconstruction toolkit (RTK). A number of algorithms are used from the analytical filtered back-projection to iterative algorithms. Imaging metrics are quantitatively assessed with a quality assurance phantom, and DECT analysis performed to determine the influence of each reconstruction technique on the relative electron density and effective atomic number values.

Chapter 4 analyses the effect of respiratory motion on optimal irradiation margins for murine lung tumour models. Four-dimensional mathematical phantoms with different lung tumour locations affected by respiratory motion and breathing curves are used for the ultimate time-resolved dose evaluated by radiotherapy metrics. An interpolation function is also investigated to reduce calculation cost.

Chapter 5 focuses on the difference in dose reporting quantities $D_{\mathrm{mm}}$ and $D_{\mathrm{wm}}$ in the $\mathrm{kV}$ photon energy range, and their conversion factors, using different approaches from cavity theory. Phantoms and an ex-vivo mouse are used for the dose calculations, where each CT voxel is considered a cavity. Calculations are performed for low- $\mathrm{kV}$ energies, where divergence among dose descriptors is augmented, and could be a limiting factor for the accurate translation of pre-clinical research into clinical trials. 
Chapter 6 proposes the use of a clinical proton platform for pre-clinical irradiations. Functionalities of the system are optimized and small fields are delivered in short irradiation times. Proton dose distributions are compared to photon dose distributions from the pre-clinical platforms discussed in this work.

Chapter 7 discusses the work described in this thesis, lists intrinsic challenges, shortcomings and the perspectives for pre-clinical external beam research in the future. 


\section{I.5 References}

[I] J.H. Breasted, The Edwin Smith Surgical Papyrus, The University of Chicago Press, Chicago, Illinois, I930.

[2] G.B. Faguet, A brief history of cancer: age-old milestones underlying our current knowledge database, Int J Cancer, I36 (2015) 2022-2036.

[3] F. Bray, J. Ferlay, I. Soerjomataram, R.L. Siegel, L.A. Torre, A. Jemal, Global cancer statistics 2018: GLOBOCAN estimates of incidence and mortality worldwide for 36 cancers in I85 countries, CA: A Cancer Journal for Clinicians, 68 (2018) 394-424.

[4] A.W. Lambert, D.R. Pattabiraman, R.A. Weinberg, Emerging Biological Principles of Metastasis, Cell, I68 (2017) 670-69I.

[5] D. Hanahan, R.A. Weinberg, Hallmarks of cancer: the next generation, Cell, I44 (201I) 646-674.

[6] J.A. Stephenson, J.C. Goddard, O. Al-Taan, A.R. Dennison, B. Morgan, Tumour Angiogenesis: A Growth Area-From John Hunter to Judah Folkman and Beyond, Journal of Cancer Research, 2013 (2013) I-6.

[7] M. Arruebo, N. Vilaboa, B. Saez-Gutierrez, J. Lambea, A. Tres, M. Valladares, A. Gonzalez-Fernandez, Assessment of the evolution of cancer treatment therapies, Cancers (Basel), 3 (2011) 3279-3330.

[8] G.P. Adams, L.M. Weiner, Monoclonal antibody therapy of cancer, Nat Biotechnol, 23 (2005) II47-II57.

[9] V. Despeignes, Observation concernant un cas de cancer de l'estomac traité par les rayons Röntgen, Journal de médecine de Lyon 82 (I896) 428-430.

[ı] K. Leszczynski, S. Boyko, On the controversies surrounding the origins of radiation therapy, Radiotherapy and Oncology, 42 (I997) 213-217.

[гі] M.J. Rivard, J.L. Venselaar, L. Beaulieu, The evolution of brachytherapy treatment planning, Med Phys, 36 (2009) 2136-2153.

[ı2] P.P. Connell, S. Hellman, Advances in radiotherapy and implications for the next century: a historical perspective, Cancer Res, 69 (2009) 383-392.

[13] F. Vernimmen, Intracranial Stereotactic Radiation Therapy With Charged Particle Beams: An Opportunity to Regain the Momentum, Int J Radiat Oncol Biol Phys, 95 (2016) $5^{2-55}$.

[I4] M.B. Sharpe, Modern Radiotherapy_Exploiting Technology Integration, Oncology \& Hematology Review (US), oo (2006).

[15] A.H. Haber, B.E. Rothstein, Radiosensitivity and Rate of Cell Division: "Law of Bergonie and Tribondeau", Science, I63 (1969) I338-I339.

[16] H.J. Muller, Artificial Transmutation of the Gene, Science, 66 (I927) 84-87. 
[r7] H.J. Muller, The effects of X Radiation on Genes and Chromossomes (Abstracts), The Anatomical Record, 37 (I927) I74.

[i8] C. Regaud, Sterilization rontgenienne totale et definitive, sans radiodermite, de testicules du belier adults; conditions de sa realisation., Compt Rend Soc de Biol., 70 (I9II) 2.

[I9] G.H. Fletcher, The evolution of the basic concepts underlying the practice of radiotherapy from I949 to I977, Radiology, I27 (I978) 3-19.

[2o] S. Rockwell, I. Dobrucki, E. Kim, S. Marrison, V. Vu, Hypoxia and Radiation Therapy: Past History, Ongoing Research, and Future Promise, Current Molecular Medicine, 9 (2009) $442-458$.

[2I] J.C. Mottram, A Factor of Importance in the Radio Sensitivity of Tumours, The British Journal of Radiology, 9 (1936) 6o6-6r4.

[22] PTCOG, Particle therapy facilities in clinical operation (last update: February 2019), 2018.

[23] M. Kemerink, T.J. Dierichs, J. Dierichs, H.J. Huynen, J.E. Wildberger, J.M. van Engelshoven, G.J. Kemerink, Characteristics of a first-generation x-ray system, Radiology, 259 (201I) 534-539.

[24] A.L. Hoffmann, H. Huizenga, J.H. Kaanders, Employing the therapeutic operating characteristic (TOC) graph for individualised dose prescription, Radiat Oncol, 8 (2013) 55 .

[25] F. Verhaegen, S. van Hoof, P.V. Granton, D. Trani, A review of treatment planning for precision image-guided photon beam pre-clinical animal radiation studies, Z Med Phys, 24 (2014) 323-334.

[26] J. Bernier, E.J. Hall, A. Giaccia, Radiation oncology: a century of achievements, Nat Rev Cancer, 4 (2004) 737-747.

[27] M. Saunders, S. Dische, A. Barrett, A. Harvey, G. Griffiths, M. Parmar, Continuous, hyperfractionated, accelerated radiotherapy (CHART) versus conventional radiotherapy in non-small cell lung cancer: mature data from the randomised multicentre trial, Radiotherapy and Oncology, 52 (1999) I37-I48.

[28] C.N. Coleman, G.S. Higgins, J.M. Brown, M. Baumann, D.G. Kirsch, H. Willers, P.G. Prasanna, M.W. Dewhirst, E.J. Bernhard, M.M. Ahmed, Improving the Predictive Value of Preclinical Studies in Support of Radiotherapy Clinical Trials, Clin Cancer Res, 22 (2016) 3138-3I47.

[29] N.L. Ford, G.K. Loudos, D.N. Karnabatidis, G.C. Kagadis, Chapter I: Defining Small Animal Imaging, Therapy, and Applications, in: G.C. Kagadis, N.L. Ford, D.N. Karnabatidis, G.K. Loudos (Eds.) Handbook of Small Animal Imaging, CRC Press, Boca Raton, FL, 2016, pp. 3-7.

[3o] R.A. Sharma, R. Plummer, J.K. Stock, T.A. Greenhalgh, O. Ataman, S. Kelly, R. 
Clay, R.A. Adams, R.D. Baird, L. Billingham, S.R. Brown, S. Buckland, H. Bulbeck, A.J. Chalmers, G. Clack, A.N. Cranston, L. Damstrup, R. Ferraldeschi, M.D. Forster, J. Golec, R.M. Hagan, E. Hall, A.R. Hanauske, K.J. Harrington, T. Haswell, M.A. Hawkins, T. Illidge, H. Jones, A.S. Kennedy, F. McDonald, T. Melcher, J.P. O'Connor, J.R. Pollard, M.P. Saunders, D. Sebag-Montefiore, M. Smitt, J. Staffurth, I.J. Stratford, S.R. Wedge, N.C.A.P.J.W. Group, Clinical development of new drug-radiotherapy combinations, Nat Rev Clin Oncol, I3 (2016) 627-642.

[3I] D.J. Rowland, S.R. Cherry, Small-animal preclinical nuclear medicine instrumentation and methodology, Semin Nucl Med, 38 (2008) 209-222.

[32] F. Verhaegen, P. Granton, E. Tryggestad, Small animal radiotherapy research platforms, Phys Med Biol, 56 (2011) R55-83.

[33] A. Zuberi, C. Lutz, Mouse Models for Drug Discovery. Can New Tools and Technology Improve Translational Power?, ILAR J, 57 (2016) I78-I85.

[34] N.E. Sharpless, R.A. Depinho, The mighty mouse: genetically engineered mouse models in cancer drug development, Nat Rev Drug Discov, 5 (2006) 74I-754.

[35] B.F. Koontz, F. Verhaegen, D. De Ruysscher, Tumour and normal tissue radiobiology in mouse models: how close are mice to mini-humans?, Br J Radiol, 90 (20I7) 2016044I.

[36] F. Verhaegen, J. Stewart, D.A. Jaffray, Chapter i8: Developing Technologies for Small Animal Radiotherapy, in: G.C. Kagadis, N.L. Ford, D.N. Karnabatidis, G.K. Loudos (Eds.) Handbook of Small Animal Imaging, CRC Press, Boca Raton, FL, 20r6, pp. 330-348.

[37] R. Clarkson, P.E. Lindsay, S. Ansell, G. Wilson, S. Jelveh, R.P. Hill, D.A. Jaffray, Characterization of image quality and image-guidance performance of a preclinical microirradiator, Med Phys, 38 (20II) 845-856.

[38] E. Tryggestad, M. Armour, I. Iordachita, F. Verhaegen, J.W. Wong, A comprehensive system for dosimetric commissioning and Monte Carlo validation for the small animal radiation research platform, Phys Med Biol, 54 (2009) 534I-5357.

[39] J. Wong, E. Armour, P. Kazanzides, I. Iordachita, E. Tryggestad, H. Deng, M. Matinfar, C. Kennedy, Z. Liu, T. Chan, O. Gray, F. Verhaegen, T. McNutt, E. Ford, T.L. DeWeese, High-resolution, small animal radiation research platform with $\mathrm{x}$-ray tomographic guidance capabilities, Int J Radiat Oncol Biol Phys, 7I (2008) I59I-I599.

[40] P.E. Lindsay, P.V. Granton, A. Gasparini, S. Jelveh, R. Clarkson, S. van Hoof, J. Hermans, J. Kaas, F. Wittkamper, J.J. Sonke, F. Verhaegen, D.A. Jaffray, Multi-institutional dosimetric and geometric commissioning of image-guided small animal irradiators, Med Phys, 4I (2014) 03I7I4. 
[4I] I.J. Chetty, B. Curran, J.E. Cygler, J.J. DeMarco, G. Ezzell, B.A. Faddegon, I. Kawrakow, P.J. Keall, H. Liu, C.M. Ma, D.W. Rogers, J. Seuntjens, D. Sheikh-Bagheri, J.V. Siebers, Report of the AAPM Task Group No. I05: Issues associated with clinical implementation of Monte Carlo-based photon and electron external beam treatment planning, Med Phys, $34(2007) 48$ I $8-4853$.

[42] L. Beaulieu, A. Carlsson Tedgren, J.F. Carrier, S.D. Davis, F. Mourtada, M.J. Rivard, R.M. Thomson, F. Verhaegen, T.A. Wareing, J.F. Williamson, Report of the Task Group I86 on model-based dose calculation methods in brachytherapy beyond the TG-43 formalism: current status and recommendations for clinical implementation, Med Phys, 39 (2012) 6208-6236.

[43] M.J. Rivard, B.M. Coursey, L.A. DeWerd, W.F. Hanson, M.S. Huq, G.S. Ibbott, M.G. Mitch, R. Nath, J.F. Williamson, Update of AAPM Task Group No. 43 Report: A revised AAPM protocol for brachytherapy dose calculations, Med Phys, 3I (2004) 633-674.

[44] M. Bazalova, J.F. Carrier, L. Beaulieu, F. Verhaegen, Tissue segmentation in Monte Carlo treatment planning: a simulation study using dual-energy CT images, Radiother Oncol, 86 (2008) 93-98.

[45] J.C. Chow, M.K. Leung, P.E. Lindsay, D.A. Jaffray, Dosimetric variation due to the photon beam energy in the small-animal irradiation: a Monte Carlo study, Med Phys, 37 (2010) $5322-5329$.

[46] K.T. Butterworth, K.M. Prise, F. Verhaegen, Small animal image-guided radiotherapy: status, considerations and potential for translational impact, Br J Radiol, 88 (2015) 20140634 .

[47] G.C. Kagadis, G. Loudos, K. Katsanos, S.G. Langer, G.C. Nikiforidis, In vivo small animal imaging: current status and future prospects, Med Phys, 37 (2010) 642I-6442.

[48] C.D. Johnstone, P. Lindsay, E.E. Graves, E. Wong, J.R. Perez, Y. Poirier, Y. BenBouchta, T. Kanesalingam, H. Chen, A.E. Rubinstein, K. Sheng, M. Bazalova-Carter, Multi-institutional MicroCT image comparison of image-guided small animal irradiators, Phys Med Biol, 62 (2017) 5760-5776.

[49] W. Huda, H.A. Kissi, K.M. Ogden, J.M. Boone, Optimizing the x-ray photon energy for digital radiographic imaging systems, SPIE2oo2.

[5o] W. van Elmpt, G. Landry, M. Das, F. Verhaegen, Dual energy CT in radiotherapy: Current applications and future outlook, Radiother Oncol, II9 (2016) I37-I44.

[5I] L.Y. Du, J. Umoh, H.N. Nikolov, S.I. Pollmann, T.Y. Lee, D.W. Holdsworth, A quality assurance phantom for the performance evaluation of volumetric micro-CT systems, Phys Med Biol, 52 (2007) 7087-7108.

[52] S. Rit, M. Vila Oliva, S. Brousmiche, R. Labarbe, D. Sarrut, G.C. Sharp, The Reconstruction Toolkit (RTK), an open-source cone-beam CT reconstruction toolkit based on the Insight Toolkit (ITK), Journal of Physics: Conference Series, 489 (2014). 
[53] M. Beister, D. Kolditz, W.A. Kalender, Iterative reconstruction methods in X-ray CT, Phys Med, 28 (2012) 94-I08.

[54] C. Bouckaert, B. Vandeghinste, C. Vanhove, S. Vandenberghe, Dual energy microCT for small animal bone-iodine decomposition, 20I2 IEEE Nuclear Science Symposium and Medical Imaging Conference Record (NSS/MIC), 20I2, pp. 3769-3774.

[55] M. Bazalova, J.F. Carrier, L. Beaulieu, F. Verhaegen, Dual-energy CT-based material extraction for tissue segmentation in Monte Carlo dose calculations, Phys Med Biol, 53 (2008) 2439-2456.

[56] M. Bazalova, E.E. Graves, The importance of tissue segmentation for dose calculations for kilovoltage radiation therapy, Med Phys, 38 (2011) 3039-3049.

[57] M. Bazalova, H. Zhou, P.J. Keall, E.E. Graves, Kilovoltage beam Monte Carlo dose calculations in submillimeter voxels for small animal radiotherapy, Med Phys, 36 (2009) 4991-4999.

[58] L.E. Schyns, I.P. Almeida, S.J. van Hoof, B. Descamps, C. Vanhove, G. Landry, P.V. Granton, F. Verhaegen, Optimizing dual energy cone beam CT protocols for preclinical imaging and radiation research, Br J Radiol, 9o (2017) 20160480.

[59] G. Landry, B. Reniers, P.V. Granton, B. van Rooijen, L. Beaulieu, J.E. Wildberger, F. Verhaegen, Extracting atomic numbers and electron densities from a dual source dual energy CT scanner: experiments and a simulation model, Radiother Oncol, Ioo (20II) $375-379$.

[6o] C.-C. Yang, J.-A. Yu, B.-H. Yang, T.-H. Wu, Optimization of the scan protocols for CT-based material extraction in small animal PET/CT studies, Nuclear Instruments and Methods in Physics Research Section A: Accelerators, Spectrometers, Detectors and Associated Equipment, 73I (2013) 299-304.

[6I] J. Seco, F. Verhaegen, (eds.), Monte Carlo Techniques in Radiation Therapy, CRC Press, Boca Raton, FL, 2013.

[62] F. Verhaegen, Chapter 20. Treatment Planning for Small Animals, in: G.C. Kagadis, N.L. Ford, D.N. Karnabatidis, G.K. Loudos (Eds.) Chapter 20. Treatment Planning for Small Animals; Handbook of Small Animal Imaging: Preclinical Imaging, Therapy, and Applications, CRC Press, Boca Raton, FL, 20I6, pp. 6ri.

[63] S.J. van Hoof, P.V. Granton, F. Verhaegen, Development and validation of a treatment planning system for small animal radiotherapy: SmART-Plan, Radiother Oncol, Iog (2013) 36I-366.

[64] S. van Hoof, J.B. Verde, F. Verhaegen, Dose painting by dynamic irradiation delivery on an image-guided small animal radiotherapy platform, The British Journal of Radiology, 92 (2019) 20180744 .

[65] T. Landberg, P. Almond, J.M.V. Burgers, M. Busch, C.A. Joslin, J.P. Paunier, Report 29, Journal of the International Commission on Radiation Units and Measurements, osi5 (1978) NP-NP. 
[66] T. Landberg, J. Chavaudra, J. Dobbs, J.P. Gerard, G. Hanks, J.C. Horiot, K.A. Johansson, T. Möller, J. Purdy, N. Suntharalingam, H. Svensson, Report 62, Journal of the International Commission on Radiation Units and Measurements, os32 (1999) NP-NP.

[67] T. Landberg, J. Chavaudra, J. Dobbs, G. Hanks, K.A. Johansson, T. Möller, J. Purdy, Report 50, Journal of the International Commission on Radiation Units and Measurements, os26 (1993) NP-NP.

[68] M. van Herk, Errors and margins in radiotherapy, Semin Radiat Oncol, I4 (2004) 5264.

[69] M.A. Hill, B. Vojnovic, Implications of respiratory motion for small animal imageguided radiotherapy, Br J Radiol, 9o (2017) 20160482.

[7o] B. van der Heyden, S.J. van Hoof, L.E. Schyns, F. Verhaegen, The influence of respiratory motion on dose delivery in a mouse lung tumour irradiation using the ${ }_{4} \mathrm{D}$ MOBY phantom, Br J Radiol, 90 (2017) 20160419.

[7I] F. Verhaegen, L. Dubois, S. Gianolini, M.A. Hill, C.P. Karger, K. Lauber, K.M. Prise, D. Sarrut, D. Thorwarth, C. Vanhove, B. Vojnovic, R. Weersink, J.J. Wilkens, D. Georg, ESTRO ACROP: Technology for precision small animal radiotherapy research: Optimal use and challenges, Radiother Oncol, I26 (2018) 47I-478.

[72] P. Andreo, Dose to 'water-like' media or dose to tissue in MV photons radiotherapy treatment planning: still a matter of debate, Phys Med Biol, 6o (2015) 309-337.

[73] A.C. Tedgren, G.A. Carlsson, Specification of absorbed dose to water using modelbased dose calculation algorithms for treatment planning in brachytherapy, Phys Med Biol, 58 (2013) 256I-2579.

[74] S.A. Enger, A. Ahnesjo, F. Verhaegen, L. Beaulieu, Dose to tissue medium or water cavities as surrogate for the dose to cell nuclei at brachytherapy photon energies, Phys Med Biol, 57 (2012) 4489-4500.

[75] E. Ford, R. Emery, D. Huff, M. Narayanan, J. Schwartz, N. Cao, J. Meyer, R. Rengan, J. Zeng, G. Sandison, G. Laramore, N. Mayr, An image-guided precision proton radiation platform for preclinical in vivo research, Phys Med Biol, 62 (2017) 43-58.

[76] C. Greubel, W. Assmann, C. Burgdorf, G. Dollinger, G. Du, V. Hable, A. Hapfelmeier, R. Hertenberger, P. Kneschaurek, D. Michalski, M. Molls, S. Reinhardt, B. Roper, S. Schell, T.E. Schmid, C. Siebenwirth, T. Wenzl, O. Zlobinskaya, J.J. Wilkens, Scanning irradiation device for mice in vivo with pulsed and continuous proton beams, Radiat Environ Biophys, 50 (2OII) 339-344.

[77] J. Constanzo, M. Fallavier, G. Alphonse, C. Bernard, P. Battiston-Montagne, C. Rodriguez-Lafrasse, D. Dauvergne, M. Beuve, Radiograaff, a proton irradiation facility for radiobiological studies at a $4 \mathrm{MV}$ Van de Graaff accelerator, Nuclear Instruments and Methods in Physics Research Section B: Beam Interactions with Materials and Atoms, 334 (2014) 52-58. 
chapter 1

[78] J. Meyer, R.D. Stewart, D. Smith, J. Eagle, E. Lee, N. Cao, E. Ford, R. Hashemian, J. Schuemann, J. Saini, S. Marsh, R. Emery, E. Dorman, J. Schwartz, G. Sandison, Biological and dosimetric characterisation of spatially fractionated proton minibeams, Phys Med Biol, 62 (2017) 9260-928I. 
Introduction and outline 



\section{CHAPTER 2}

The impact of dual energy CT imaging on dose calculations for pre-clinical studies

Ana Vaniqui, Lotte EJR Schyns, Isabel P Almeida, Brent van der Heyden, Stefan J van Hoof, Frank Verhaegen

Radiation Oncology

November $2017,12: 181$ 


\section{Abstract}

Background: To investigate the feasibility of using dual-energy CT (DECT) for tissue segmentation and kilovolt $(\mathrm{kV})$ dose calculations in pre-clinical studies and assess potential dose calculation accuracy gain.

Methods: Two phantoms and an ex-vivo mouse were scanned in a small animal irradiator with two distinct energies. Tissue segmentation was performed with the single-energy CT (SECT) and DECT methods. A number of different material maps was used. Dose calculations were performed to verify the impact of segmentations on the dose accuracy.

Results: DECT showed better overall results in comparison to SECT. Higher number of DECT segmentation media resulted in smaller dose differences in comparison to the reference. Increasing the number of materials in the SECT method yielded more instability. Both modalities showed a limit to which adding more materials with similar characteristics ceased providing better segmentation results, and resulted in more noise in the material maps and the dose distributions. The effect was aggravated with a decrease in beam energy. For the ex-vivo specimen, the choice of only one high dense bone for the SECT method resulted in large volumes of tissue receiving high doses. For the DECT method, the choice of more than one kind of bone resulted in lower dose values for the different tissues occupying the same volume. For the organs at risk surrounded by bone, the doses were lower when using the SECT method in comparison to DECT, due to the high absorption of the bone. SECT material segmentation may lead to an underestimation of the dose to OAR in the proximity of bone.

Conclusions: The DECT method enabled the selection of a higher number of materials thereby increasing the accuracy in dose calculations. In phantom studies, SECT performed best with three materials and DECT with seven for the phantom case. For irradiations in pre-clinical studies with $\mathrm{kV}$ photon energies, the use of DECT segmentation combined with the choice of a low-density bone is recommended. 


\section{I Background}

Pre-clinical radiation studies with small animal models play a significant role in the understanding of cancer radiobiology. Such studies also aim towards mimicking human treatment capabilities so that specific validated radiation therapies in animal models can be successfully translated into patient radiotherapy (RT) trials ${ }^{[\mathrm{I}]}$. Accurate pre-clinical radiation targeting requires accurate image guiding. For the various stages of target delineation, treatment planning, dose calculation, beam delivery, and subsequent outcome assessments, precise identification of different tissues and structures is of paramount importance.

Computed Tomography (CT) is the most frequently used imaging modality for $\mathrm{RT}^{[2]}$. Commercial pre-clinical irradiators are equipped with an $\mathrm{x}$-ray tube, which besides the irradiation, is used to acquire high-resolution cone beam CT (CBCT) images (about Ioo-200 $\mu \mathrm{m})^{[3]}$.

Small animal irradiation is preferably performed with kilovolt $(\mathrm{kV})$ photons ${ }^{[4]}$, in contrast to human radiotherapy which is mostly performed with megavolt (MV) photons. In the $\mathrm{kV}$ energy range, the photo-electric effect is increasingly important and its interaction probability is strongly dependent on the effective atomic number of the tissues $\left(Z_{\text {eff }}^{3^{2} 4}\right)^{[3]}$. In current practice, quantitative information on tissues is mostly obtained by single energy CT (SECT) in the form of attenuation coefficients (or CT numbers, expressed by Hounsfield Units, HU). In Monte Carlo (MC) dose calculations, every voxel of the CT scan has a mass density assigned based on the HU value through an empirical calibration.

Tissue identification based on SECT has been shown to lead to errors in dose calculations in the $\mathrm{kV}-\mathrm{MV}$ energy range ${ }^{[5]}$ and due to the strong dependence of the photoelectric cross sections on the atomic number of the tissues, such errors are amplified in the low-energy photon range ${ }^{[6]}$. In addition, dose calculation algorithms for $\mathrm{kV}$ irradiations of small animals need supplementary information to voxel densities, such as tissue type - as it cannot be assumed the medium is water in $\mathrm{kV}$ irradiations. This information can be provided from either SECT or dual energy CT (DECT) images.

The aim of this study is to investigate the feasibility of using dual-energy CBCT for tissue segmentation and $\mathrm{kV}$ dose calculations in pre-clinical studies. The main objectives are to assess potential dose calculation accuracy 
gain from DECT and to establish imaging protocols that allow accurate dose calculations.

While this work has no direct clinical implications, its underlying aim is to perform dose calculations as accurately as possible so as to enable rigorous subsequent clinical translation.

\subsection{Methods}

\subsection{Micro irradiator}

The X-RAD ${ }_{225}$ Cx (Precision X-Ray, North Branford (CT), United States) ${ }^{[4,7]}$ micro irradiator consists of a dual-focus $\mathrm{X}$-ray tube with a maximum tube potential of $225 \mathrm{kV}$ (225 Cx, Comet, Switzerland) and a $20^{\circ}$ angled tungsten stationary target. The $\mathrm{X}$-ray tube acts as photon source for imaging using the small focal spot, and treatment using the large focal spot. Photons are filtered through a $0.8 \mathrm{~mm}$ beryllium exit window and additional $2.0 \mathrm{~mm}$ filter cassette made of aluminium for imaging or $0.32 \mathrm{~mm}$ filter cassette made of copper ${ }^{[8]}$ for irradiation purposes. The source to isocentre distance was fixed at $303.6 \mathrm{~mm}$.

\subsubsection{Extracting information from SECT and DECT methods}

For this study, two geometrically identical cylindrical mini-phantoms (SmART Scientific Solutions BV, Maastricht, the Netherlands) of $3 \mathrm{~cm}$ diameter and I cm length were scanned (2.I(a)). They are composed of a Solid Water bulk and twelve cylindrical inserts of $3.5 \mathrm{~mm}$ diameter and I $\mathrm{cm}$ length. The composition of the inserts, the relative electron density $\left(\rho_{\mathrm{e}}\right)^{\mathrm{r}}$ and the effective atomic number $\left(Z_{\text {eff }}\right)^{2}$ provided by the manufacturer are listed in Table 2.I. The phantom cross-section is consistent with the overall size of the mouse, both head and pelvis, further used in this study.

The mini phantoms were imaged using the CBCT imager (resolution I024XIO24 pixels) integrated in the small animal irradiator. The images were acquired using a $2.0 \mathrm{~mm}$ filter of aluminium for the tube voltages of $50 \mathrm{kVp}$ (low energy) and $90 \mathrm{kVp}$ (high energy) with corresponding currents of 5.59 and $2.08 \mathrm{~mA}$ (Figure 2.I(e) shows both photon spectra).

${ }^{1} \rho_{\mathrm{e}}=(\mathrm{NA} \rho Z / \mathrm{A}) /\left(\mathrm{NA}_{\mathrm{w}} Z_{\mathrm{w}} / \mathrm{A}_{\mathrm{w}}\right)$, where NA is the Avogadro's number, $\rho, Z$ and $\mathrm{A}$ are the mass density, atomic number, and atomic mass of a material, while the subscript $\mathrm{w}$ indicates water

${ }^{2} Z_{\text {eff }}=\left(\Sigma_{i} w_{i} Z_{i}^{\beta}\right)^{1 / \beta}$, where wi is the weight fraction of element $i$ with atomic number $Z_{i}$ and $\beta=3.3{ }^{[6]}$ 
The exposures used were of $670.8 \mathrm{mAs}$ and $249.6 \mathrm{mAs}$ yielding the dose of $30 \mathrm{cGy}$ for each energy. The absorbed dose to water at the phantom surface was verified using a TN3oor2 Farmer ionization chamber (PTW, Freiburg, Germany) according to the AAPM TG-6I protocol for $40-300 \mathrm{~V}$ $\mathrm{x}$-ray beam dosimetry dosimetry (in-air calibration method) ${ }^{[9]}$. The images were reconstructed using a Feldkamp-Davis-Kress (FDK) backprojection algorithm ${ }^{[\mathrm{ro}]}$, in a matrix of $34 \mathrm{IX} 324 \times 96$ with $\mathrm{I0} 3.4 \times 103.4 \times 103.4 \mu \mathrm{m}^{3}$ voxel size. The acquisition time difference between the two images was of seven minutes.
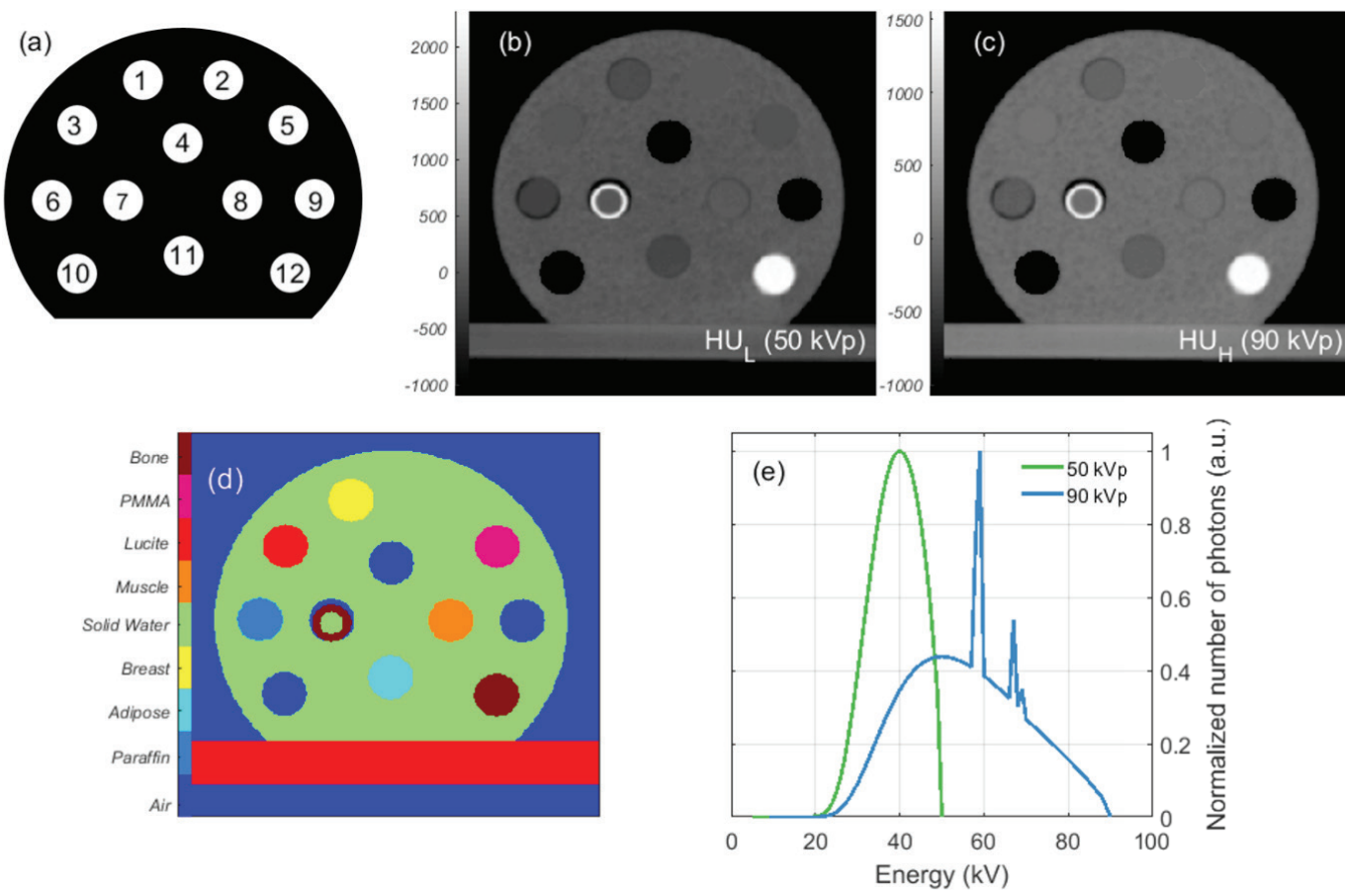

Figure 2.I - (a) Phantoms are made of Solid Water and contain twelve inserts of tissue-equivalent materials, one set of materials for the calibration phantom and one set of materials for the validation phantom. (b) Central slice of the CT scan at $50 \mathrm{kVp}$ and (c) $90 \mathrm{kVp}$. (d) Reference material map. (e) $50 \mathrm{kVp}$ and $90 \mathrm{kVp}$ photon spectra used for SECT and DECT. 


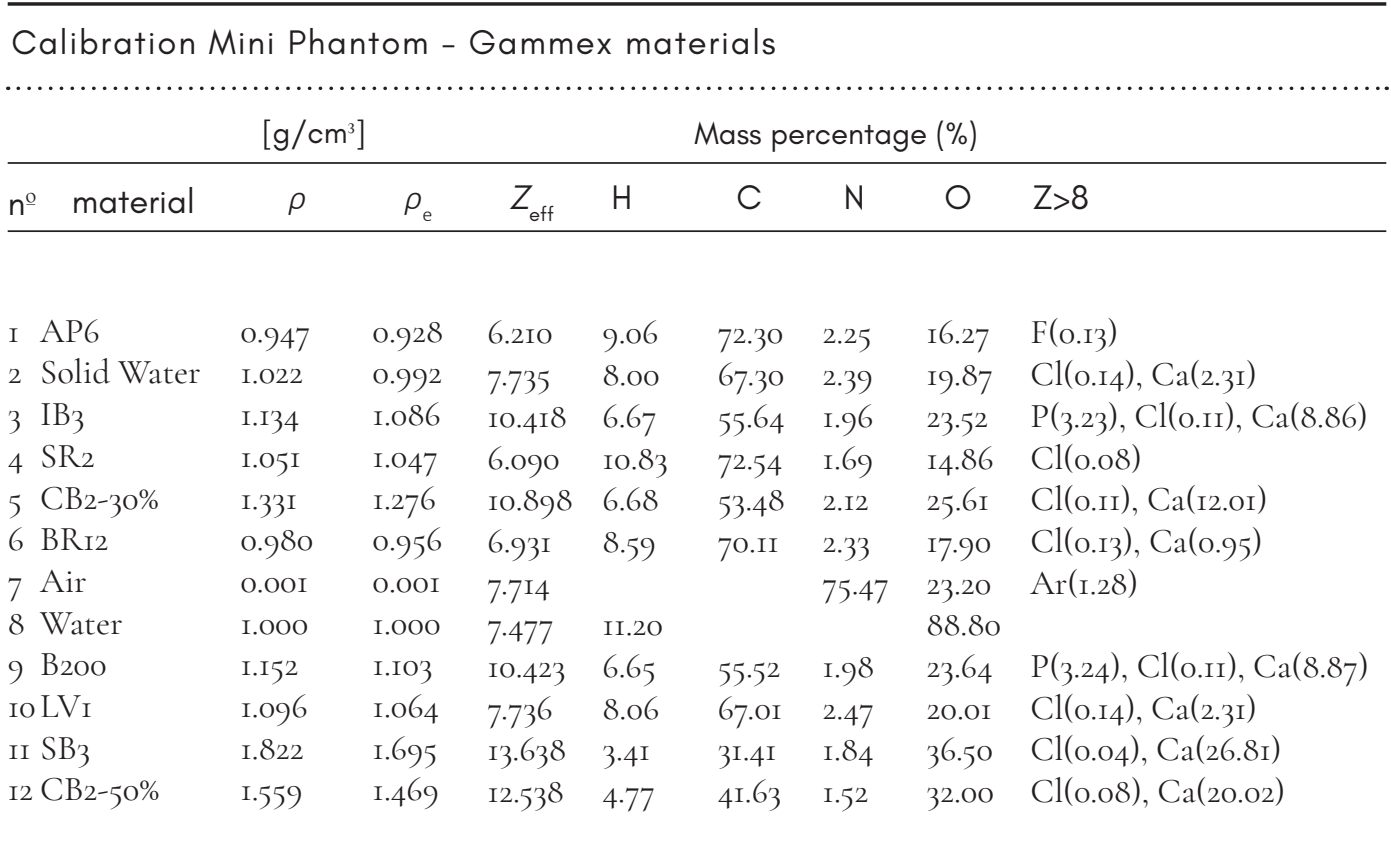

Validation Mini Phantom - CIRS, Gammex and other materials

\begin{tabular}{|c|c|c|c|c|c|c|c|c|}
\hline \multirow[b]{2}{*}{$\mathrm{n}^{\mathrm{o}}$ material } & \multicolumn{2}{|c|}{$\left[\mathrm{g} / \mathrm{cm}^{3}\right]$} & \multicolumn{5}{|c|}{ Mass percentage $(\%)$} & \multirow[b]{2}{*}{$Z>8$} \\
\hline & $\rho$ & $\rho_{\mathrm{e}}$ & $Z_{\text {eff }}$ & $\mathrm{H}$ & C & $\mathrm{N}$ & ○ & \\
\hline I BRi2 & 0.980 & 0.956 & $6.93 \mathrm{I}$ & 8.59 & 7O.II & 2.33 & 17.90 & $\mathrm{Cl}(0.13), \mathrm{Ca}(0.95)$ \\
\hline 2 Teflon & 2.153 & I. 860 & $8.46 \mathrm{I}$ & & 24.00 & & & $\mathrm{~F}(76.00)$ \\
\hline 3 Lucite & I.I 80 & I.I46 & 6.529 & 8.05 & $59 \cdot 98$ & & 31.96 & \\
\hline 4 Air & O.OOI & O.OOI & 7.714 & & & $75 \cdot 47$ & 23.20 & $\operatorname{Ar}(\mathrm{I} .28)$ \\
\hline 5 PMMA & I.I9O & 1.156 & 6.529 & 8.05 & $59 \cdot 98$ & & 31.96 & \\
\hline 6 Paraffin & 0.930 & 0.959 & 5.483 & $\mathrm{I} 4.90$ & 85.10 & & & \\
\hline 7 Water & I.OOO & I.0OO & $7 \cdot 477$ & II. 20 & & & 88.80 & \\
\hline 8 Muscle & I.062 & I.04I & 7.588 & 9.10 & 69.70 & 2.10 & I6.80 & $\mathrm{Cl}($ o.ro $), \mathrm{Ca}(2.20)$ \\
\hline 9 Air & O.OOI & O.OOI & $7 \cdot 714$ & & & 75.47 & 23.20 & $\operatorname{Ar}(\mathrm{I} .28)$ \\
\hline IO Air & O.OOI & O.OOI & $7 \cdot 7 \mathrm{I} 4$ & & & 75.47 & 23.20 & $\operatorname{Ar}(\mathrm{I} .28)$ \\
\hline II Adipose & 0.967 & 0.956 & 6.439 & IO.OO & 71.30 & I. $8 \mathrm{O}$ & I6.40 & $\mathrm{Cl}(0.20), \mathrm{Ca}(0.30)$ \\
\hline I2 Bone & I.600 & I. 507 & II. 895 & 4.83 & 37.03 & 0.97 & 35.66 & $\mathrm{Mg}(6.19), \mathrm{Cl}(0.05), \mathrm{Ca}\left(\mathrm{I}_{5} .24\right)$ \\
\hline
\end{tabular}

Table 2.I - Reference values of mass density $(\rho)$, relative electron density $\left(\rho_{\mathrm{e}}\right)$, effective atomic number $\left(Z_{\text {eff }}\right)$ and elemental composition of the tissue-substitute materials present in the calibration and validation mini-phantoms. 


\subsubsection{SECT method}

In the SECT approach, a relationship between HU and mass density $(\rho)$ was generated in the form of a $(H U-\rho)$ calibration curve. $H U$ are defined as $H U=\operatorname{Iooo}\left(\mu / \mu_{W}-\mathrm{I}\right)$, where $\mu$ and $\mu_{\mathrm{W}}$ are respectively the linear attenuation coefficients of the scanned medium and water. Relative electron density $\rho_{\mathrm{e}}$ can be converted into mass density $\rho$ through a linear relationship. A piecewise bi-linear $H U-\rho$ relationship was generated using the mean $H U$ values of the selected materials in the calibration phantom (Figure 2.2). The material segmentation is indicated with vertical lines according to the selected $H U$ ranges. Figure 2.3 shows the histogram of Hounsfield Units. From the $(H U-\rho)$ calibration curve, a density map of the phantom was created. A density to material curve was derived from the density map, which generated the material map. The curve material thresholds were set based on visual inspection of the CT scan as well as on the knowledge of the maximum and minimum $H U$ of each material. In this example, seven materials were chosen for the segmentation. Table 2.2 shows the mean $H U$ values for each material. A density map was then generated and, according to the chosen segmentation intervals, a material map was generated.

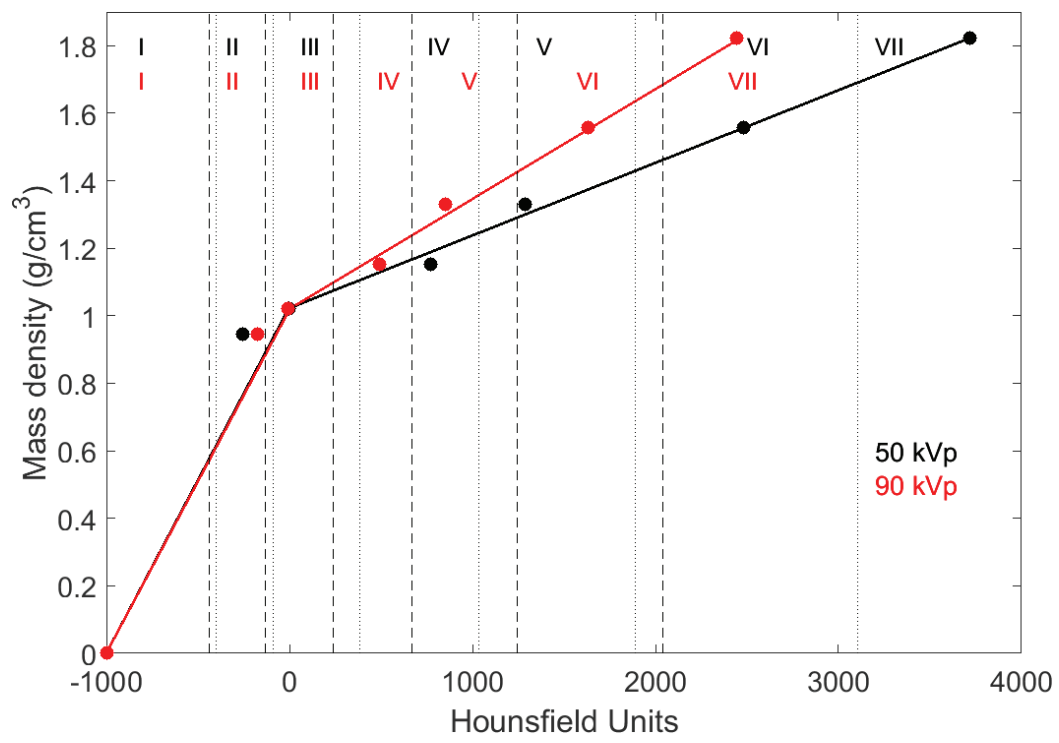

Figure 2.2 - SECT (HU- $\rho$ ) curve for the calibration phantom at $50 \mathrm{kVp}$ in black and at $90 \mathrm{kVp}$ in red. The vertical dotted $(50 \mathrm{kVp})$ and dashed lines $(90 \mathrm{kVp})$ represent the selected boundaries between media in a segmentation scheme with seven materials (I to VII). The roman numerals I-VII indicate the materials: air, AP6, Solid Water, B200, $\mathrm{CB}_{2}-30 \%, \mathrm{CB}_{2}-50 \%$ and $\mathrm{SB}_{3}$. Other segmentation schemes with a different number of intervals are possible. The dots represent the mean HU value of each material. 


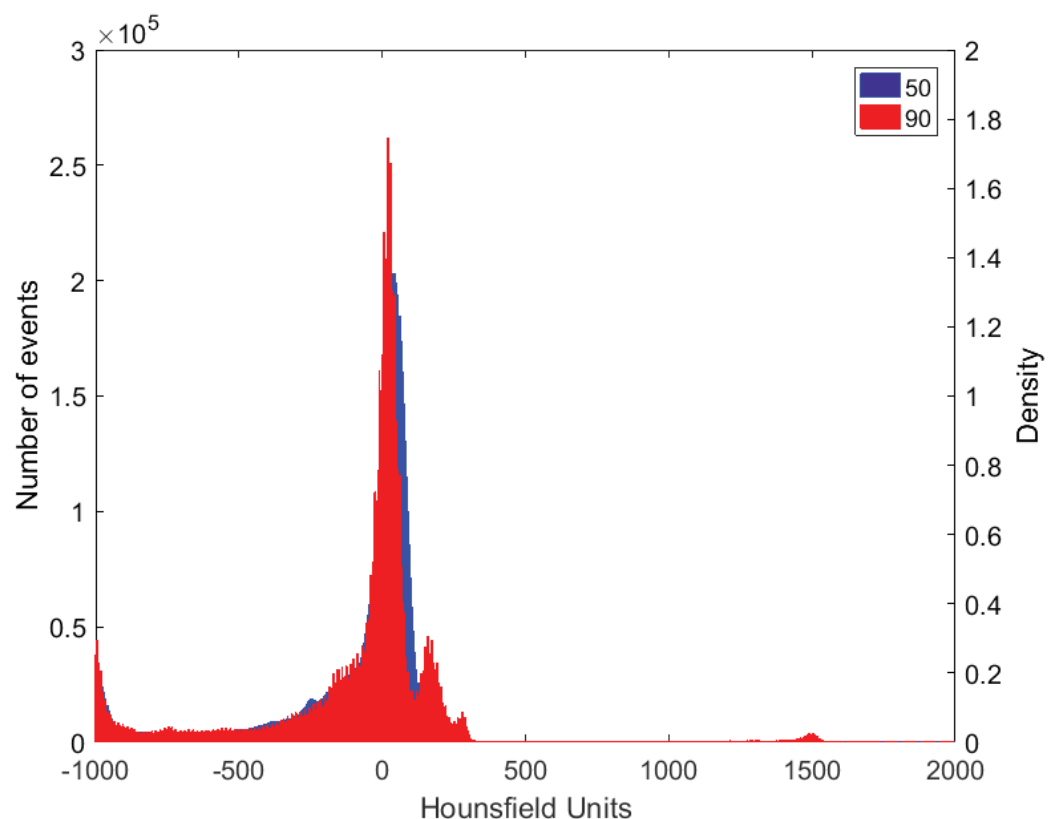

Figure 2.3 - Hounsfield Units histogram for 50 and $90 \mathrm{kVp}$

\begin{tabular}{|c|c|c|c|c|c|c|c|}
\hline \multicolumn{4}{|c|}{ Calibration phantom } & \multicolumn{4}{|c|}{ Validation phantom } \\
\hline \multirow{2}{*}{ Material } & \multicolumn{2}{|c|}{ Mean $\mathrm{HU} \pm \sigma$} & \multirow{2}{*}{$\begin{array}{l}\text { Insert } \\
\text { № }\end{array}$} & \multirow{2}{*}{ Material } & \multicolumn{2}{|c|}{ Mean $\mathrm{HU} \pm \sigma$} & \multirow{2}{*}{$\begin{array}{l}\text { Insert } \\
\text { № }\end{array}$} \\
\hline & $50 \mathrm{kVp}$ & $90 \mathrm{kVp}$ & & & $50 \mathrm{k} \vee p$ & $90 \mathrm{kVp}$ & \\
\hline Air & $-1000 \pm 19$ & $-\mathrm{IOOO} \pm \mathrm{I} 3$ & 7 & Air & $-\mathrm{IOOO} \pm \mathrm{I} 7$ & $-\mathrm{IOOO} \pm \mathrm{I} 3$ & $4,9, \mathrm{IO}$ \\
\hline $\mathrm{AP} 6$ & $-248 \pm 17$ & $-I 73 \pm I 6$ & I & Paraffin & $-28 \mathrm{I} \pm \mathrm{I} 3$ & $-\mathrm{I} 78 \pm \mathrm{II}$ & 6 \\
\hline $\mathrm{BR}_{\mathrm{I} 2}$ & $-17 \mathrm{I} \pm 19$ & $-\mathrm{II} 5 \pm \mathrm{I} 6$ & 6 & CIRS Adipose & $-226 \pm 16$ & $-I 47 \pm I 3$ & II \\
\hline $\mathrm{SR}_{2}$ & $-147 \pm 18$ & $-64 \pm 15$ & 4 & BRI2 & $-146 \pm 16$ & $-106 \pm I 3$ & I \\
\hline Solid Water & $-2 \pm 22$ & $-7 \pm 20$ & 2 & PMMA & $-73 \pm \mathrm{I} 2$ & $\mathrm{IO} \pm \mathrm{II}$ & 5 \\
\hline Water & $\mathrm{O} \pm 22$ & $\mathrm{O} \pm \mathrm{I} 7$ & 8 & Water & $-36 \pm I 5$ & $-\mathrm{I} 5 \pm \mathrm{I} 2$ & 7 \\
\hline LVI & $69 \pm 23$ & $68 \pm 20$ & IO & Lucite & $-3 \mathrm{I} \pm \mathrm{I} 3$ & $46 \pm \mathrm{I} 2$ & 3 \\
\hline $\mathrm{IB}_{3}$ & $729 \pm 54$ & $45^{6} \pm 38$ & 3 & Muscle & $2 \pm I 4$ & $25 \pm \mathrm{I} 2$ & 8 \\
\hline $\mathrm{B} 200$ & $768 \pm 38$ & $482 \pm 3 I$ & 9 & CIRS Bone & $2230 \pm 29$ & $1508 \pm 22$ & $\mathrm{I} 2$ \\
\hline $\mathrm{CB}_{2}-30 \%$ & $\mathrm{I} 297 \pm 22$ & $857 \pm 16$ & 5 & & & & \\
\hline $\mathrm{CB}_{2}-50 \%$ & $2483 \pm 28$ & $\mathrm{I} 632 \pm \mathrm{I} 6$ & I2 & & & & \\
\hline $\mathrm{SB}_{3}$ & $3723 \pm 25$ & $2448 \pm 17$ & II & & & & \\
\hline
\end{tabular}

Table 2.2 - Mean $H U \pm$ standard deviation $(\sigma)$ per insert for the calibration and validation phantoms for 50 and $90 \mathrm{kVp}$. 
The tissue segmentation, i.e. the process of assigning tissue type and mass density to each voxel, was performed with the SECT image (either the 50 or the $90 \mathrm{kVp}$ scan) and the calibration curve, a two-segment linear relationship (HU- $\rho$ ), shown on Figure 2.2. Different SECT segmentation schemes were derived based on three, four or seven materials to evaluate the effect of the number of media on the segmentation - see Table 2.3.

\subsubsection{DECT method}

For DECT, the CT numbers were extracted from circular regions of interest of the inserts in the four central slices of the high energy $\left(\mathrm{HU}_{\mathrm{H}}\right)$ and the low energy $\left(H U_{\mathrm{L}}\right)$ scans. The procedure described by Schyns et $a^{\left[{ }^{[\mathrm{II}]}\right.}$ to determine the $\rho_{\mathrm{e}}$ values, using Saito's ${ }^{[\mathrm{r}]}$ approach, and to extract $Z_{\text {eff }}$, following the method proposed by Landry et al ${ }^{\left[{ }^{[3]}\right]}$, was adopted. From the $\mathrm{HU}_{\mathrm{L}}$ and $\mathrm{HU}_{\mathrm{H}}$ images, $Z_{\mathrm{eff}}$ and $\rho_{\mathrm{e}}$ maps were derived and used for the tissue segmentation. Figure 2.4 shows the relationship between $Z_{\text {eff }}$ and $\rho_{\mathrm{e}}$ for the materials of the validation phantom. Mass densities were assigned based on the $\rho_{\mathrm{e}}$ images using the $\left(\rho, \rho_{\mathrm{e}}\right)$ relationship $\left(\rho=\mathrm{I} .073 \rho_{\mathrm{e}}-\mathrm{0.04}, \mathrm{R}^{2} \geq\right.$ 0.999), the linear relationship between $\rho$ and $\rho_{\mathrm{e}}$ was found by fitting the data (least squares method) for the insert materials listed on Table 2.I. All voxels to which no $Z_{\text {eff }}$ value could be assigned, predominantly located at sharp transitions between air and the solid water bulk, were excluded from the analysis (<0.0I\% in the regions of interest).
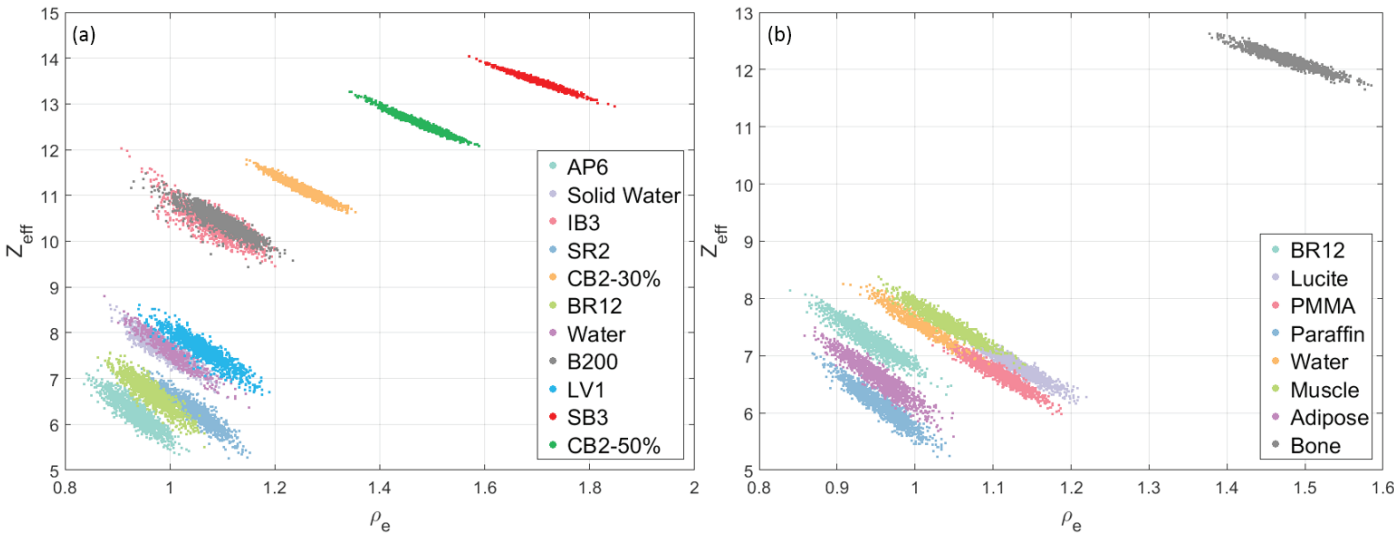

Figure 2.4 - DECT tissue segmentation for all voxels of the (a) calibration and (b) validation phantoms. 
The tissue segmentation was performed with the reconstructed $\rho_{\mathrm{e}}$ and $Z_{\mathrm{eff}}$ of each voxel. The DECT scans at $50 \mathrm{kVp}$ and $90 \mathrm{kVp}$ were used, as this combination has shown to be optimal in terms of $Z_{\text {eff }}$ and $\rho_{\mathrm{e}}$ errors for the $X-R A D$ system with the $3 \mathrm{~cm}$ phantoms ${ }^{[\mathrm{IrI}]}$. The reference values of $Z_{\mathrm{eff}}$ and $\rho_{\mathrm{e}}$, named $Z_{\mathrm{R}}$ and $\rho_{\mathrm{R}}$, and the calculated values from the DECT images, named $Z_{\mathrm{C}}$ and $\rho_{\mathrm{C}}$, were used to assign tissue composition to a voxel. The distance vector between $\mathrm{A}=\left[Z_{\mathrm{R}}, \rho_{\mathrm{R}}\right]$ and $\mathrm{B}=\left[Z_{\mathrm{C}}, \rho_{\mathrm{C}}\right]$ was calculated and the reference tissue minimizing the distance length was assigned to the voxel. The Mahalanobis distance was used as it is less affected by imaging noise, following the method described by Landry et al. ${ }^{[6]}$

Different DECT segmentation schemes were also investigated with seven, eight and nine materials. Table 2.3 shows the schemes.

\subsubsection{Reference Phantom}

A reference phantom serves as standard for the material segmentation and the dose calculations. It is a mathematical structure created with thresholds and masks for each phantom. It has a single reference value for each material property. The material assignment to the reference phantom is indicated in Figure 2.I(a), according to Table 2.I.

Figure 2.I (d) shows the nine materials used, with densities ranging from o.oor to I. $6 \mathrm{~g} / \mathrm{cm}^{3}$ (air - bone). In all phantom cases in this study, a broad beam impinges on the phantom from the right-left direction and encompasses its entire volume. The dose is normalized to the maximum dose value in the reference phantom.

All the results are compared to the segmentation and the dose calculation of the reference phantom.

\subsubsection{Ex-vivo mouse specimen}

An ex-vivo male mouse was imaged and the same procedures previously described for DECT and SECT, including the calibration phantom parameters, were applied to its CT scans and dose calculations. A region comprising the head of the mouse was selected for this study and material maps with six tissues for DECT and three tissues for SECT were created based on the ICRU Report $44^{\left[{ }_{4}\right]}$ tissues, listed in Table 2.4. Using Landry's method, we chose the closest ICRU tissues to the selected specimen, instead of the materials from the phantom inserts. A fictitious tumour was delineat- 
ed in a region partially comprising the brain and another organ-at-risk (OAR), the spinal cord. Table 2.3 also shows the segmentation schemes for SECT and DECT.

The tumour, brain, bone and OAR regions are illustrative structures to investigate possible differences between imaging methods.

\begin{tabular}{|c|c|c|c|}
\hline \multicolumn{4}{|c|}{ Validation phantom } \\
\hline № & \multicolumn{3}{|c|}{ Reference } \\
\hline 9 & \multicolumn{3}{|c|}{ Air, Adipose, Brain, Spongiose, Cranium, Cortical Bone } \\
\hline № & SECT & № & DECT \\
\hline 3 & Air, Solid Water, Bone & 7 & $\begin{array}{l}\text { Air, Paraffin, Adipose, } \\
\text { Breast, Solid Water, Bone }\end{array}$ \\
\hline 4 & Air, Adipose, Muscle, Bone & 8 & $\begin{array}{l}\text { Air, Paraffin, Adipose, Breast, } \\
\text { Solid Water, Muscle, Lucite, Bone }\end{array}$ \\
\hline 7 & $\begin{array}{l}\text { Air, Paraffin, Adipose, Breast, } \\
\text { Solid Water, Lucite, Bone }\end{array}$ & 9 & $\begin{array}{l}\text { Air, Paraffin, Adipose, Water, Breast, } \\
\text { Solid Water, Muscle, Lucite, Bone }\end{array}$ \\
\hline \multicolumn{4}{|c|}{ Ex-vivo Mouse } \\
\hline 4 & Air, Brain, Cortical Bone & 6 & $\begin{array}{l}\text { Air, Adipose, Brain, Spongiosa, } \\
\text { Cranium, Cortical Bone }\end{array}$ \\
\hline
\end{tabular}

Table 2.3 - Different segmentation schemes for SECT and DECT for the validation phantom: SECT was segmented with three, four and seven number of materials. For DECT, the segmentation was performed with seven, eight or nine materials. For the ex-vivo mouse, SECT was segmented with three materials and DECT with six.

Mouse - ICRU Tissues

\begin{tabular}{|c|c|c|c|c|c|c|c|c|c|}
\hline \multirow{2}{*}{ № } & \multirow{2}{*}{ Material } & \multirow{2}{*}{$\frac{\left[\mathrm{g} / \mathrm{cm}^{3}\right]}{\rho}$} & \multicolumn{7}{|c|}{ Mass percentage $(\%)$} \\
\hline & & & $\rho_{\mathrm{e}}$ & $Z_{\text {eff }}$ & $\mathrm{H}$ & C & $\mathrm{N}$ & $\mathrm{O}$ & $Z>8$ \\
\hline I & Adipose & 0,93 & $0,95 \mathrm{I}$ & $6,42 \mathrm{I}$ & II, 60 & 68, I0 & 0,20 & 19,80 & $\mathrm{Na}$ (o.I), S(o.I), $\mathrm{Cl}($ o.I $)$ \\
\hline 2 & Brain & $\mathrm{I}, \mathrm{O} 4$ & $\mathrm{I}, \mathrm{O} 35$ & 7,578 & I0,70 & 14,50 & 2,20 & $7 \mathrm{I}, 20$ & $\mathrm{Na}(0.2), \mathrm{P}(0.4), \mathrm{S}(\mathrm{o.2}), \mathrm{Cl}(0.3), \mathrm{K}(0.3)$ \\
\hline 3 & Spongiosa & $\mathrm{I}, \mathrm{I} 8$ & $\mathrm{I}, \mathrm{I} 5 \mathrm{I}$ & IO, 230 & 8,50 & 40,40 & 2,80 & 36,70 & $\begin{array}{l}\mathrm{Na}(\text { o.I }), \mathrm{Mg}(0.2), \mathrm{P}(3.4), \mathrm{S}(\mathrm{o.2}), \mathrm{Cl}(\mathrm{o.2}), \\
\mathrm{K}(\mathrm{o.I}), \mathrm{Ca}(7.4), \mathrm{Fe}(\text { o.I })\end{array}$ \\
\hline 4 & Cranium & $\mathrm{I}, 6 \mathrm{I}$ & $\mathrm{I}, 5 \mathrm{I} 7$ & I2,709 & 5,00 & $2 \mathrm{I}, 2 \mathrm{O}$ & $4, \mathrm{oO}$ & 43,50 & $\mathrm{Na}(0 . \mathrm{I}), \mathrm{Mg}(0.2), \mathrm{P}(8 . \mathrm{I}), \mathrm{S}(0.3), \mathrm{Ca}(\mathrm{I} 7.6)$ \\
\hline 5 & Cortical Bone & $\mathrm{I}, 9^{2}$ & $\mathrm{I}, 780$ & I3, 629 & 3,40 & 15,50 & 4,20 & 43,50 & $\mathrm{Na}($ o.I $), \mathrm{Mg}($ о.2), $\mathrm{P}($ го. 3$), \mathrm{S}($ о.3), $\mathrm{Ca}(22.5)$ \\
\hline 6 & Air & 0,0012 & o, оог & 7,714 & & & 75,47 & 23,20 & $\operatorname{Ar}(\mathrm{I} .28)$ \\
\hline
\end{tabular}

Table 2.4 - Tissue data from the ICRU Report $44^{\left[{ }_{4}\right]}$ for the mouse segmentation. 


\subsubsection{Dose Calculations}

After the segmentations procedures based on SECT and DECT, dose calculations were performed to verify the impact of these segmentations on the dose accuracy.

The dedicated small animal radiotherapy planning system SmART-Plan (research version I.5, Precision X-ray, North Branford, CT, United States) was used to calculate the dose distributions ${ }^{\left[{ }_{15}\right]}$. The dose engine used by SmART-Plan is the MC code EGSnrc/DOSXYZnre ${ }^{[16-18]}$. The first step was to provide the material datasets for subsequent use by EGSnrc. Photons were transported down to an energy cutoff (PCUT) of Io $\mathrm{keV}$ and the electron energy cutoff (ECUT) was set to a total energy value of $736 \mathrm{keV}$ (225 kV kinetic energy, meaning no secondary electrons were transported). The photon spectra for the irradiation were calculated using SpekCal ${ }^{[19,20]}$ for 100,160 , and $225 \mathrm{kVp}$, according to the X-ray tube parameters. Exclusively for the ex-vivo mouse simulations, phase-space files for $225 \mathrm{kVp}$ and roo $\mathrm{kVp}$ with a $5 \mathrm{~mm}$ beam diameter were used, preserving the abovementioned characteristics. For the phantom dose calculations, broad beams that covered the phantom were used.

Geometry input files for the phantoms and the animal specimen were created with a Matlab 2016a (The Mathworks, Natick, MA, United States) routine according to the SECT or DECT material segmentation.

The mass density values of liquid and solid water differed only by $2.2 \%$, therefore Solid Water was solely used in both phantoms. For the calibration phantom, material maps were made either using Liver and Inner Bone or Brain and Bone Mineral, and the remaining media, due to the proximity in density values. For the validation phantom, the insert Teflon was not used and Lucite and PMMA were regarded as Lucite, once more due to their similar compositions. Different material maps were also investigated to achieve a better segmentation using fewer media.

The planned dose to water was set to 2 Gy at the isocentre and the number of MC histories with no particle recycling used to achieve a $3 \%$ statistical uncertainty for dose calculations with I03.4 x I03.4 x I03.4 $\mu \mathrm{m}^{3}$ voxels was set to $5 \cdot \mathrm{IO}^{9}$ photons for the mini phantoms. The beam field size was set to $3.5 \times \mathrm{I} \mathrm{cm}$, comprising the selected region of the mini phantom completely. For the mouse, two parallel opposed beams, at $29^{\circ}$ and $209^{\circ}$, and $9 \cdot \mathrm{IO}^{7}$ particles were used, achieving I\% statistical uncertainty for a dose of 2 Gy at isocentre. 


\subsection{Results}

\subsection{SECT Segmentation - number of materials, $225 \mathrm{kVp}$ irradiation spectrum}

Unless stated otherwise, the results presented in this section were generated using the validation phantom. Figure 2.5 shows the effect of the different numbers of SECT segmentation materials on the MC dose calculations.

The dose to the bulk region of SECT with three materials, SECT3, segmentation agrees with the reference within $\pm \pm 5 \%$ on average. The steps in the profile are due to the Lucite inserts assigned in the Reference phantom but absent in SECT3, their dose differences are $20 \pm \mathrm{I} \%$ (Figure 2.5(c)). Figure 2.7 shows the difference with respect to the reference for all inserts in each SECT scenario.

To increase the efficiency of the dose calculations, no dose was scored in air, thence the regions with zero dose surrounding the phantom and in the air insert.

A different behaviour is shown for the four media segmentation, $\mathrm{SECT}_{4}$, (Figures 2.5(e-h)). Using materials with densities slightly lower (Adipose, $0.967 \mathrm{~g} / \mathrm{cm}^{3}$ ) and higher (Muscle, $\left.1.062 \mathrm{~g} / \mathrm{cm}^{3}\right)$ than Solid Water (I.022 g/ $\mathrm{cm}^{3}$ ), the bulk of the phantom is assigned as Muscle, and the inserts Breast, Paraffin and partially the PMMA, are assigned as Adipose. The phantom's bulk dose differs by $\pm \pm \pm \%$ from the reference and in the inserts, Lucite has the highest difference, $34 \%$, followed by lower differences in the remaining inserts (Figure 2.7). This clearly shows that SECT tissue segmentation is very sensitive to a slight change in the number of materials, and that the selected HU intervals can significantly influence the dose calculations for the $\mathrm{kV}$ photon range.

For the seven-material segmentation, SECT7 (Figure 2.5(i)) the misassignment of media has a noise-like appearance in the material and dose maps and profiles (Figures 2.5(i-1)). The material map of $\mathrm{SECT}_{7}$ has $72 \%$ of its materials correctly assigned. Regarding the dose, an agreement of $3 \pm 5 \%$ for the bulk was found and the highest dose difference was once more in Lucite, 2 r\%. It should be stressed that due to the misassignment of media small dose spikes are present throughout the geometry. Assigning a larger number of materials clearly introduces noise in the media assignment and the dose calculations, and the choice of $\mathrm{HU}$ intervals becomes also more arbitrary.

For the three cases, Air and Bone are always correctly segmented. 

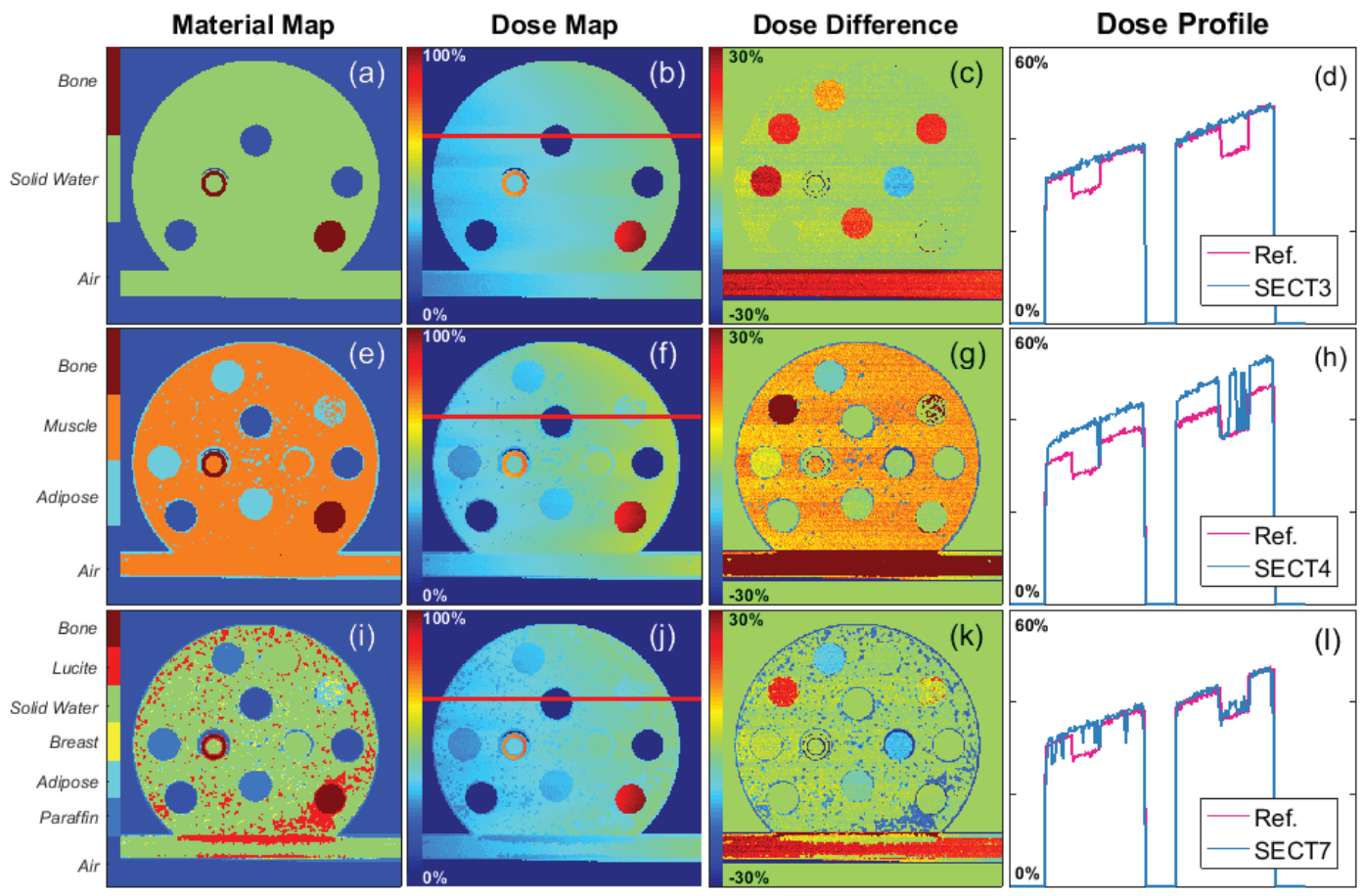

Figure 2.5 - Material maps, dose maps, dose difference, and dose profiles for different SECT segmentation schemes at $50 \mathrm{kVp}$ and the $225 \mathrm{kVp}$ irradiation spectrum. (a) Three, (e) four, and (i) seven different media were used in the three rows. The dose profiles (d), (h) and (1) were obtained from the red line in the images $(b),(f)$ and $(j)$. Images $(c),(g)$, and $(k)$ show the dose difference comparison $(\Delta D)$ with the reference, where $\Delta \mathrm{D}=[($ DSECT-DRef $) / \mathrm{DRef}] \cdot$ 'го\%. The dose maps are normalized to the maximum dose of the reference dose map. The material maps should be compared to the reference phantom, Figure 2.I (d).

Different material combinations were tested besides the reported ones. The choice for $\mathrm{SECT}_{3}$ and $\mathrm{SECT}_{4}$ was based on the current pre-clinical practice, and $\mathrm{SECT}_{7}$ is shown for further comparison with $\mathrm{DECT}_{7} . \mathrm{A}$ higher number of SECT materials is not reported as seven fell beyond the limits of the method. The Hounsfield Units histogram, Figure 2.3, shows that with a limited number of peaks, a limited number of materials can be assigned using SECT. Another dimension becomes necessary to discern more materials, such as the $\rho_{\mathrm{e}}-Z_{\mathrm{eff}}$ space in DECT.

\subsubsection{DECT Method, $225 \mathrm{kVp}$ irradiation spectrum}

For the DECT segmentation, maps with seven (DECT7), eight (DECT8), and nine materials (DECT9) were tested (Figure 2.6 (a), (e), (i)). Similar as SECT, a number of material combinations were tested. The reported DECT combinations were selected based on the highest separation between 
relative electron density and effective atomic number values, and increased accuracy on the segmentation in comparison to the reference.

Increasing the number of materials does not automatically imply a better segmentation for DECT, similar as for SECT. The media misassignment, over $52 \%$ for DECT8 and $54 \%$ for DECT9, again exhibits noise in the dose maps and profiles (Figure 2.6 (b), (f), (j), (d), (h), (l)) with small dose spikes. The material map of DECT 7 was only I $6 \%$ in disagreement with the reference. For DECT8 and DECT9, the dose difference in the bulk region
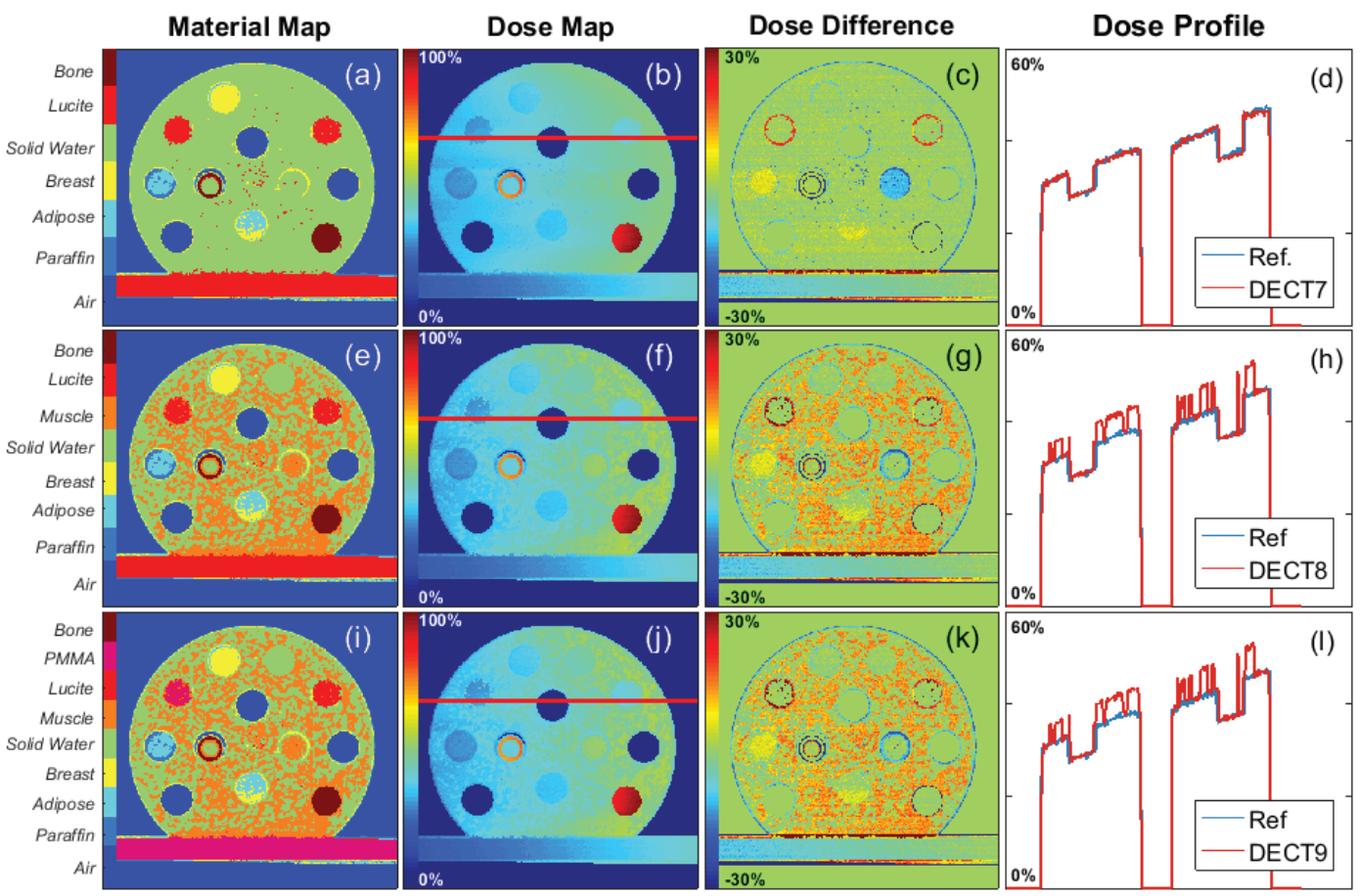

Figure 2.6 - Material maps, dose maps, dose difference and dose profiles for different DECT segmentation schemes and the $225 \mathrm{kVp}$ irradiation spectrum. (a) Seven, (e) eight and (i) nine different media were used in the three rows. The dose profiles (d), (h) and (l) were obtained from the red line in the images $(b),(f)$ and $(j)$. Images $(c),(g)$, and $(k)$ show the dose comparison $(\Delta D)$ with reference, where $\Delta D=[(D-$ DECT-DRef)/Dref]-IOO\%. The dose maps are normalized to the maximum dose of the reference dose map. The material maps should be compared to the reference phantom, Figure 2.I (d).

Figure 2.7 shows that dose differences relative to the reference phantom are much higher for the SECT segmentations in comparison to the DECT ones. For kilovolt energies, DECT segmentation yields better results, increasing the dose calculation accuracy when compared to the SECT method. 


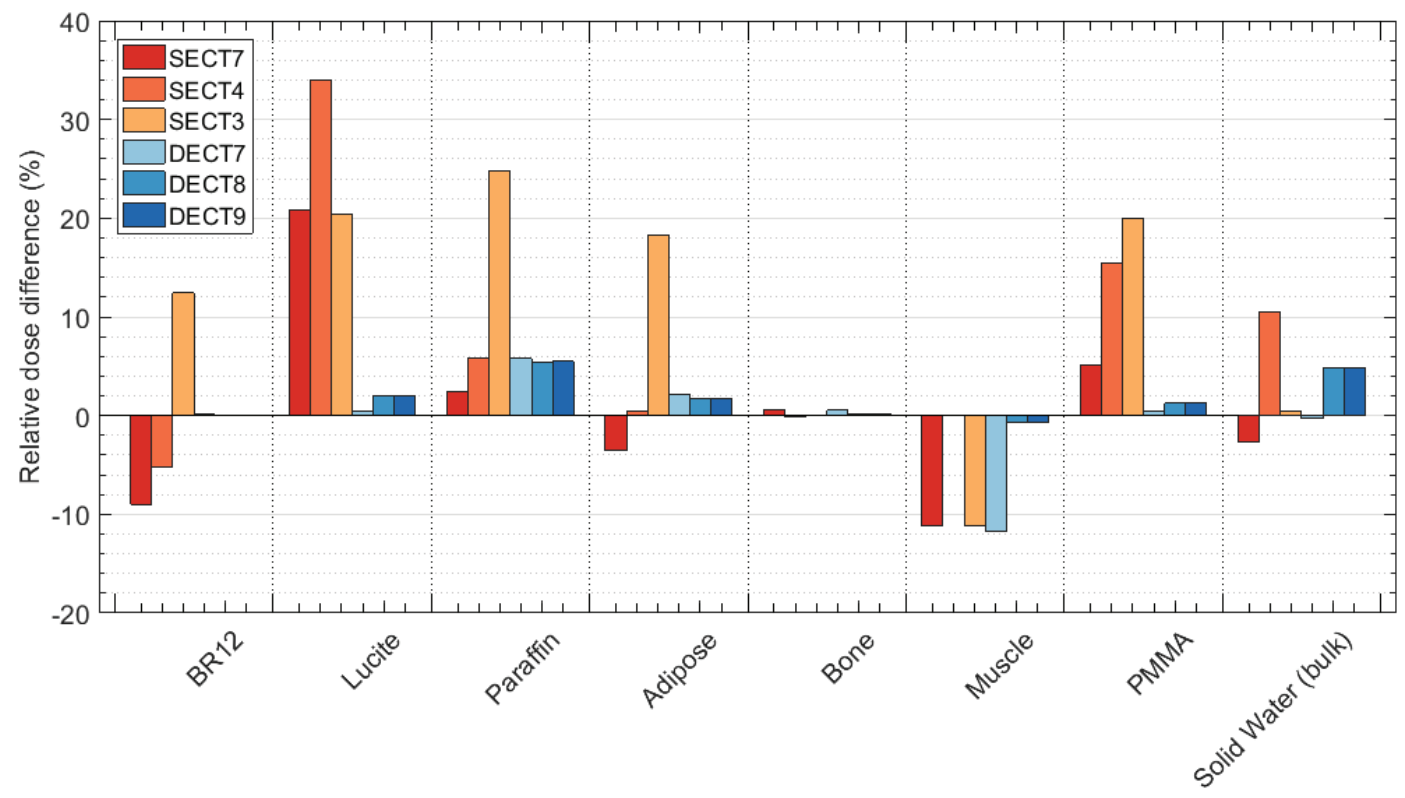

Figure 2.7 - Relative dose difference between the mean doses calculated per insert (and for the bulk of the phantom) of the reference and for SECT and DECT segmentations: SECT3, SECT4, SECT7, $\mathrm{DECT}_{7}, \mathrm{DECT}$, and DECT9. Regions of interest were defined avoiding boundary regions.

is, on average, of $5 \pm 6 \%$ higher than the reference. The insert materials were mostly correctly assigned in the three cases. Figure 7 shows that the highest difference is for the material Muscle in DECT7, I2 $\pm \mathrm{I} \%$ - Muscle is not one of the media segmented in DECT 7 . From figures $2.6(\mathrm{c}),(\mathrm{g}),(\mathrm{k})$ it is clear that the tissue segmentation scheme may influence the dose accuracy. It should be noted that for DECT the highest dose differences are concentrated in the boundary regions.

\subsubsection{Additional irradiation spectra}

In addition to the $225 \mathrm{kVp}$ spectrum, roo and $\mathrm{i} 60 \mathrm{kVp}$ photon beams were used for the dose calculations. In Figure 2.8, a histogram shows the errors on the insert dose values for the SECT and DECT methods of each spectrum. The higher the frequency of events in the zero dose-error bar, the better the segmentation method performed for a specific imaging energy. 


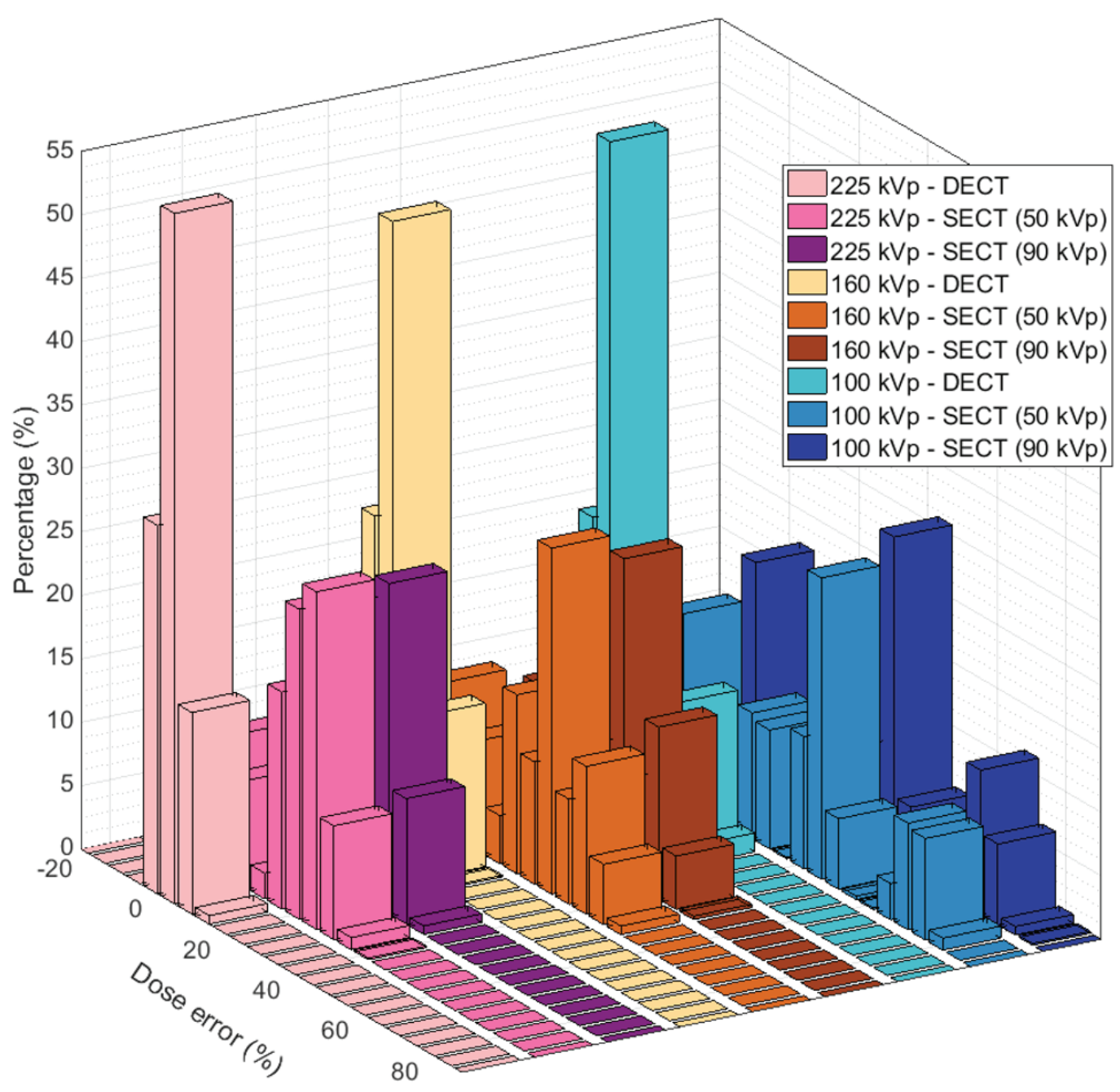

Figure 2.8 - Histogram of the dose disagreement with the reference in the insert regions for DECT, SECT5o and SECT9o at 225, 160 and roo $\mathrm{kVp}$. 
Overall, the $225 \mathrm{kVp}$ spectrum presented the best results, followed by the I6o $\mathrm{kVp}$ and the roo $\mathrm{kVp}$. Furthermore, for the three spectra, the DECT method performed better, the zero dose error contained 54\%, 50\% and 53\% of the voxels for the 225 , 160 and roo $\mathrm{kVp}$ spectra, respectively, and there were no differences higher than $\mathrm{I} 7 \%$, for the 225 and $\mathrm{i} 6 \mathrm{o} \mathrm{kVp}$ and $27 \%$ for the roo $\mathrm{kVp}$ beam. For SECT at $50 \mathrm{kVp}$, SECT5o, the dose differences were as high as $37 \%, 52 \%$ and $82 \%$, and at $90 \mathrm{kVp}$, SECT9o, they were as high as $33 \%, 52 \%$ and $82 \%$, for the 225 , 160 and roo $\mathrm{kVp}$ spectra respectively.

\subsubsection{Ex-vivo mouse}

In this section the emphasis is on the difference between the dose calculations based on the two imaging methods as it was not possible to produce a reference ex-vivo mouse - it would require precise knowledge of all its tissues and structures. Although material and dose differences in the bulk of the phantom were shown in the previous section, this concept does not apply to the specimen, as there is no bulk of the mouse.

The SECT (SECT5O and SECT9o) and DECT segmentation schemes as shown in Table 2.3 were used. The choice for three media for SECT was based on current pre-clinical practice using 3-4 media ${ }^{[3,21-25]}$ and the phantom results of Section 2.I. For DECT, six tissues with differences in $\rho_{e}(>\mathrm{II} \%)$ and $Z_{\text {eff }}(>\mathrm{I} 8 \%)$ were chosen as section 2.2 showed the DECT method to have superior results in the presence of media with a degree of separation in these quantities.

Figures 2.9 (a-c) show the axial, coronal and sagittal views of the delineated head of the mouse. The green region in Figure 2.9(a) indicates the position of the parallel-opposed beams. The elliptical green areas in Figures 2.9 (b-c) indicate the target volume used for the dose calculations, it encompasses the tumour, which is partially in the brain and the spinal cord. The dose to the target was set to 2 Gy. Figures 2.9 (d-e), show higher doses for the SECT map, whereas the DECT dose map reveals a gradient due to the presence of different bone media in the same volume. The choice of only one kind of bone implicates a high dose for the different media assigned as Cortical Bone in the SECT method. Figures $2.9(\mathrm{f}-\mathrm{g})$ show the dose ratio of SECT and DECT dose maps with accentuated dose differences in Adipose, e.g. close to the outer skin, and in Bone, which are more pronounced for the roo $\mathrm{kVp}$ beam $(5.0 \%$ of the all voxels in the body contour showed ratios higher than 4 ). 

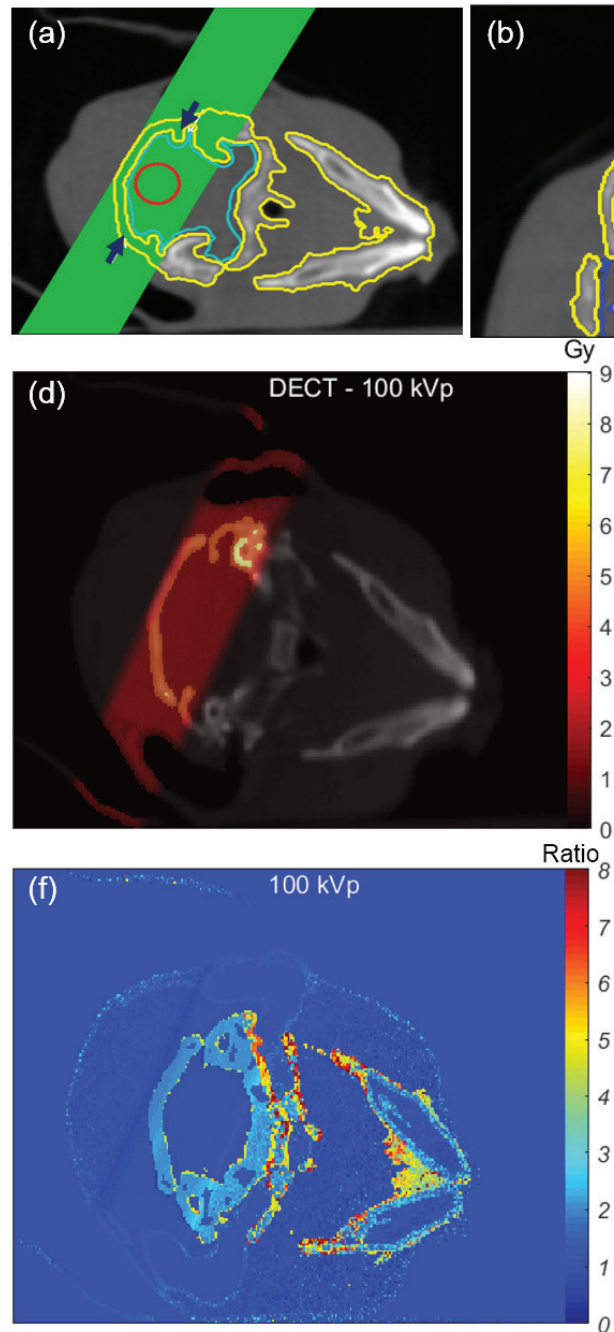
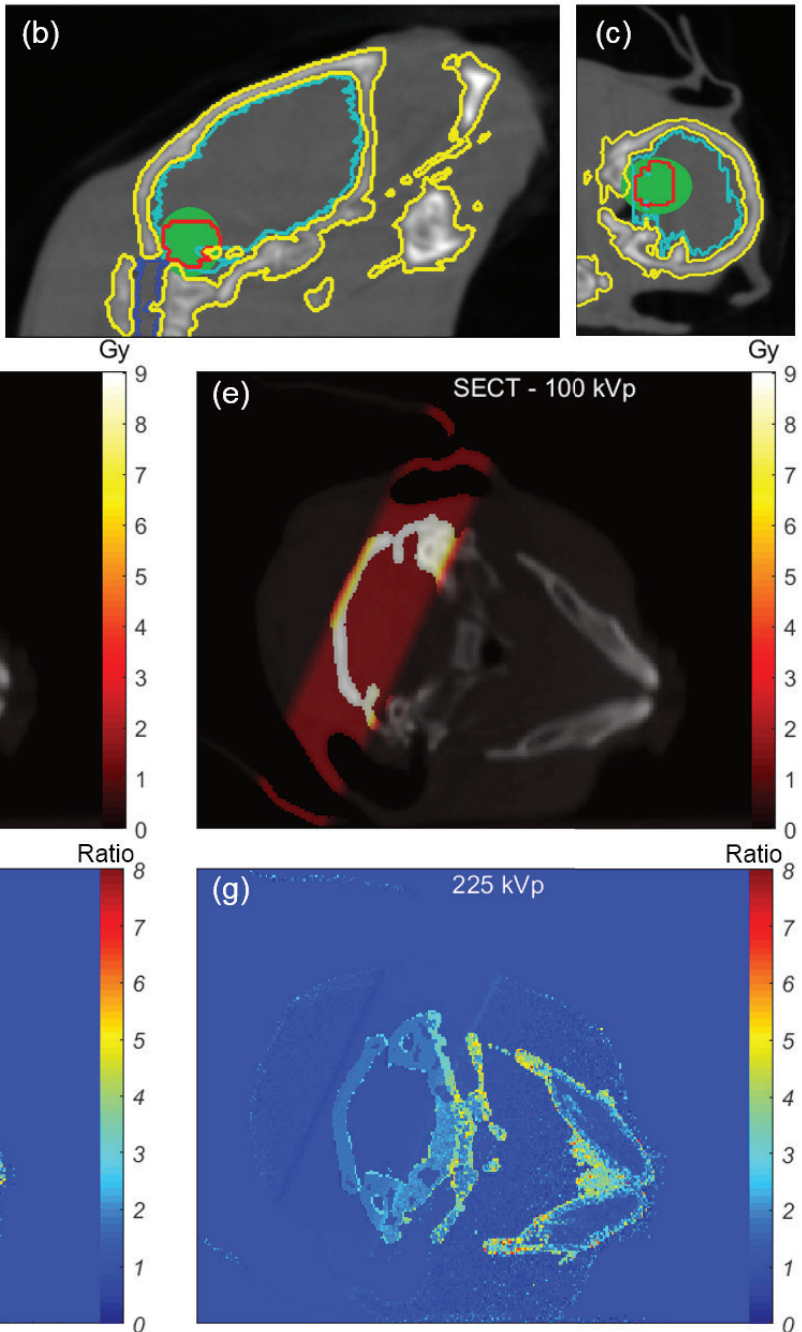

Figure 2.9 - (a) Axial, (b) sagittal and (c) coronal views of the delineated head of the ex-vivo mouse. The green region in (a) and the arrows indicate the beams used for the dose calculations. It encompasses the fictitious tumour (red contour), which is partially in the brain (light blue contour) and the spinal cord (dark blue contour). The elliptic green regions in (b-c) indicate the target region for the simulation. Figures (d), (e), show the roo $\mathrm{kVp}$ dose map for DECT and SECT5o, and Figures (f), (g) show the ratio between SECT and DECT dose maps for Ioo and $225 \mathrm{kVp}$ beams. Due to the similarities between SECT50 and SECT9o, only the SECT50 case is shown here.

Another way of quantifying the impact of the different segmentations is through the Dose Volume Histograms (DVHs). Figures 2.Io (a-b) show the DVHs for the roo and $225 \mathrm{kVp}$ beams. For the bone contour, the dose reaches values three to five times higher than the prescription dose for the 225 and the roo $\mathrm{kVp}$ beams, respectively. The maximum dose was $63 \%$ higher for the 
Ioo $\mathrm{kVp}$ beams, respectively. The maximum dose was $63 \%$ higher for the Ioo $\mathrm{kVp}$ beam in comparison to the $225 \mathrm{kVp}$ one. For roo $\mathrm{kVp}$, the presence of higher dose regions is due to a steeper dose gradient required to reach the target value (2 Gy) in the prescription point, for which the same coordinates were specified for the roo and $225 \mathrm{kVp}$ beams.

Regarding the segmentation method, the SECT curve presents a smooth and steady behaviour as it was segmented with only one type of bone. The DECT curve presents three plateau regions for doses higher than 2 Gy. For Ioo $\mathrm{kVp}$, the first region ends with a slope approximately at $4.2 \mathrm{~Gy}$, the second at 7.8 Gy and the last one reaches the maximum dose of 9.5 Gy, and for $225 \mathrm{kVp}$, the same behaviour is shown at 3.2, 5.0 and 5.9 Gy. It indicates the presence of different bone types used in DECT: Spongiosa, Cranium and Cortical Bone.



Figure 2.IO - DVHs for the (a) IOo and (b) $225 \mathrm{kVp}$ beams. Four structures were delineated (as shown in Figure 2.9 (a-c)): Brain, Spinal Cord, Tumour and Bone. The same contours were utilized for all simulations. The solid and the dash-dot lines indicate the DVHs for the DECT and the SECT50 method, respectively. 


\begin{tabular}{|c|c|c|c|c|c|c|c|c|}
\hline & \multicolumn{2}{|c|}{$100 \mathrm{kVp}$} & \multirow{2}{*}{\multicolumn{2}{|c|}{$\begin{array}{c}225 \mathrm{kVp} \\
\mathrm{DECT} \approx \mathrm{SECT}\end{array}$}} & \multicolumn{2}{|c|}{$100 \mathrm{kVp}$} & \multicolumn{2}{|c|}{$225 \mathrm{kVp}$} \\
\hline & DECT & SECT & & & DECT & SECT & SECT & SECT \\
\hline Dose (Gy) & \multicolumn{4}{|c|}{ Brain } & \multicolumn{4}{|c|}{ Spinal Cord } \\
\hline Mean & 0.3 & 0.3 & 0.3 & 0.3 & 0.3 & 0.3 & 0.6 & 0.5 \\
\hline Maximum & 2.3 & 2.3 & $2 . \mathrm{I}$ & $2 . \mathrm{I}$ & 2.3 & 2.0 & 2.0 & 2.0 \\
\hline D95 & 0.0 & 0.0 & 0.0 & 0.0 & 0.0 & o.o & 0.0 & o.o \\
\hline $\mathrm{D}_{5}$ & 2.1 & 2.0 & 2.0 & I.9 & 2.0 & I.9 & I. 7 & I.9 \\
\hline DI & $2 . \mathrm{I}$ & 2.0 & $2 . \mathrm{I}$ & 2.0 & 2.0 & I.9 & 2.0 & I.9 \\
\hline Dose (Gy) & \multicolumn{4}{|c|}{ Tumour } & \multicolumn{4}{|c|}{ Bone } \\
\hline Mean & $2 . \mathrm{I}$ & 2.0 & 2.0 & 2.0 & 0.3 & 0.7 & 0.3 & 0.6 \\
\hline Maximum & $2 . \mathrm{I}$ & $2 . \mathrm{I}$ & $2 . \mathrm{I}$ & $2 . \mathrm{I}$ & 9.5 & 9.8 & $5 \cdot 9$ & 6.0 \\
\hline D95 & 2.0 & I.9 & 2.0 & 2.0 & 0.0 & 0.0 & 0.o & 0.0 \\
\hline $\mathrm{D}_{5}$ & $2 . \mathrm{I}$ & 2.0 & $2 . \mathrm{I}$ & 2.I & 3.0 & 8.3 & 3.0 & 5.5 \\
\hline DI & $2 . \mathrm{I}$ & 2.0 & $2 . \mathrm{I}$ & $2 . \mathrm{I}$ & 4.4 & $9 \cdot 3$ & 3.2 & 5.8 \\
\hline
\end{tabular}

Table 2.5 - For each combination of beam energy and imaging method the mean and maximum dose values, the dose values on $95 \%, 5 \%$ and $\mathrm{I} \%$ of the volume (D95, $\mathrm{D}_{5}$ and $\mathrm{D}_{\mathrm{I}}$ ).

The higher energy absorption in bone owing to the exclusive use of the dense Cortical Bone in SECT results in lower doses for the Spinal Cord DVH curves, a structure inside vertebras. Table 2.5, shows the minimum dose to the hottest $\mathrm{I} \%\left(\mathrm{D}_{\mathrm{I}}\right), 5 \%\left(\mathrm{D}_{5}\right)$ and 95\% (D95) to provide additional information on the uniformity of the dose. The $\mathrm{D}_{5}$ and $\mathrm{D}_{\mathrm{I}}$ values for Brain and Tumour are $5 \%$ lower for SECT in relation to DECT for both energies. The use of SECT with only one type of bone yielded larger volumes with high doses and the bone choice influenced the dose received by the other structures. 


\subsection{Discussion}

This study showed the high impact of incorrect material segmentation on the dose calculation accuracy for $\mathrm{kV}$ photon beams employed in small animal irradiators, using the different imaging modalities: SECT and DECT. The effect is aggravated with a decrease in beam energy, due to the increase in the importance of the photo-electric effect with decreasing photon energy, causing materials with different effective atomic numbers to absorb increasingly different fractions of energy in photon beams. For irradiations with photon spectra below roo $\mathrm{kVp}$, the differences would even be more pronounced.

Although broadly used, there are still a few caveats regarding the SECT method. It is not clear which media should be used for generating the calibration curve and the number of linear segments as well as the position of the tissue boundaries is arbitrary and difficult to establish manually using the HU histogram ${ }^{[3]}$.

DECT showed better overall results in comparison to SECT. The higher number of DECT segmentation media resulted in smaller dose differences in comparison to the reference (Figure 2.7) for the phantom cases. Increasing the number of materials in the SECT method yielded more instability, in addition to being a method that has a higher degree of arbitrariness in tissue assignment than DECT. Material boundaries have to be selected based on the distribution of $\mathrm{HU}$, and include a visual inspection of the segmentation result (i.e. in an overlap plot of the CT and the material map), which indicates inter-individual differences may result. Both modalities have a limit to which adding more materials with similar characteristics ceased providing better segmentation results, and resulted in more noise in the material maps and the dose distributions.

For the mouse case, the choice of Cortical Bone for the SECT method, as is common practice in the literature, resulted in large volumes of tissue receiving high doses. For the DECT method, the choice of more than one kind of bone resulted in lower dose values for the different tissues occupying the same volume, only I.9\% of the bone tissues in DECT were assigned as Cortical Bone (I $8.5 \%$ as Cranium and $79.6 \%$ as Spongiosa). For the OAR surrounded by bone in the beam path, the doses were lower when using the SECT method in comparison to DECT, due to the high absorption of the Cortical Bone and the hardening of the beam (low-energy photons were absorbed in the bone), resulting in less photoelectric interactions and hence 
dose deposition in the bone ${ }^{[25]}$. Therefore, SECT material segmentation may lead to an underestimation of the dose to OAR in the proximity of bone (other examples could be organs in the pelvic area or close to the thoracic spine). In view of these results, with the assumption that bones in small animals might not be as dense or with such elevated atomic number as human bones and considering the interest in studies with lower energies, it can be recommended not to use Cortical Bone when performing SECT segmentation. The choice of Spongiosa would be more appropriate and additional bone types may need to be considered for specific regions, as mouse bones are very flexible, in composition possibly closer to human cartilage, which has less phosphorus and calcium than Cortical Bone. For studies with lower energies, the choice becomes more important if higher doses to bony structures are not intended. It is also beneficial to employ harder beam filters.

DECT with three or four tissues is not reported. The method's advantage lies in the possibility of exploring different segmentations based on higher number of tissues. A reduced number of materials would not benefit this site.

In the soft tissue range, the benefits of DECT for the energy $225 \mathrm{kVp}$ are relatively small. For small animals such as mice, the affected regions lie mainly in bony structures. For bigger animals, cumulative errors could have a larger role and need further considerations. Improvements in tissue segmentation from DECT are needed for lower photon energies and proton beams in all tissues.

A source of uncertainty in this study is the presence of noise in the CT scans. In Figure 2.I (b-c), artefacts can be seen in the bone insert, and the bulk of the phantom seems to have a texture instead of consisting of a uniform medium. The CT values of the entire region are irregular, $42 \pm 62$ and $\mathrm{I} 6 \pm 57 \mathrm{HU}$ for 50 and $90 \mathrm{kVp}$ scans. For DECT, the $Z_{\text {eff }}$ image is the most affected, with a noisy appearance and the bulk medium with a mean $Z_{\text {eff }}$ value of $8.0 \pm 0.4$ (ranging from 6.0 to I0.7), which encompasses many of the soft tissues used in the segmentation and makes it especially hard to distinguish between Water, Solid Water and Muscle, which also have densities close together. The large misassignment of materials on DECT8 and DECT9, using materials with similar characteristics $\left(Z_{\text {eff }}\right.$ and $\left.\rho_{\mathrm{e}}\right)$ can be partially attributed to image noise. The image noise and misassignment follow a similar pattern on Figures 2.6(f) and (j). The CT projections were 
reconstructed with a simple FDK backprojection algorithm. The usage of an iterative reconstruction algorithm with beam hardening and artefact correction kernels could improve the effect of noise on the images and provide superior material segmentation when performing DECT ${ }^{[26]}$.

The boundary regions of the phantom and the inserts presented the highest source of errors for DECT. This can be explained as a partial volume effect: as two contiguous materials partially fill a voxel, they are combined into voxels that do not correspond to the CT numbers of either of the materials. This will play a larger effect in phantoms with small air gaps than in animals. Another possible and complementary explanation is that the images should have a perfect overlap with the reference phantom, a small misregistration would provide substantial differences. This is a feature DECT is sensitive to, while it plays no role for SECT images. For small shifts between two scans, due to setup or animal movement, rigid image registration could be used if potential HU errors from interpolations are minimal.

Dose calculations in human radiotherapy in the megavoltage photon energy range are not very sensitive to tissue compositions, however, in the $\mathrm{kV}$ range used in brachytherapy ${ }^{[6]}$ and in pre-clinical studies mimicking human radiotherapy at the level of rodents it becomes a potential cause of uncertainties $^{[22]}$. A final issue that deserves attention is that in the present study and, in general, the small animal radiobiology literature, specimens are segmented with human-like tissues. It is reasonable to assume that either knowing the actual composition or deriving a relationship between human and animal tissues should benefit the dose calculation accuracy and the absorbed dose for the photon energies used in this study. 


\subsection{Conclusions}

The feasibility of dual-energy CBCT imaging for $\mathrm{kV}$ dose calculations in pre-clinical studies was presented. Images obtained using well-separated $\mathrm{X}$-ray spectra were acquired with an on-board imager and different segmentation schemes tested. The DECT method enabled the employment of a higher number of materials increasing accuracy in dose calculations. In phantom studies, both SECT and DECT presented a limit to which adding materials resulted in more imaging noise in the material maps and the dose distributions. SECT performed best with three materials and DECT with seven for the phantom case. With lower beam energies, the effect of incorrect segmentation on the dose calculations was worse, due to the importance of the photoelectric effect for the $\mathrm{kV}$ energy range. DECT segmentation offers the distinct advantage of taking into consideration the effective atomic number of the media. For the ex-vivo specimen, the dose calculations derived from the SECT method showed larger volumes with high doses. For $\mathrm{kV}$ energies, the use of DECT segmentation combined with the choice of a bone with low density and atomic number is recommended.

\section{Acknowledgements}

The authors would like to thank Blake Walters, Dr Mark Podesta, Dr Gabriel Fonseca, and Relinde Lieverse for useful discussions. 


\subsection{References}

${ }^{[\mathrm{r}]}$ Koontz BF, Verhaegen F, De Ruysscher D. Tumour and normal tissue radiobiology in mouse models: how close are mice to mini-humans? Br. J. Radiol. 2016;2016044I.

${ }^{[2]}$ Grau C, Defourny N, Malicki J, Dunscombe P, Borras JM, Coffey M, et al. Radiotherapy equipment and departments in the European countries: Final results from the ESTROHERO survey. Radiother. Oncol. 2014;II2:I55-64.

[3] Verhaegen F, van Hoof S, Granton P V, Trani D, Hoof S Van, Granton P V, et al. A Review of Treatment Planning for Precision Image-Guided Photon Beam Preclinical Animal Radiation Studies. Z. Med. Phys. 2014;24:323-34.

${ }_{44]}$ Verhaegen F, Granton P, Tryggestad E. Small animal radiotherapy research platforms. Phys. Med. Biol. 20II;56:R55-83.

[5] Bazalova M, Carrier J-F, Beaulieu L, Verhaegen F. Dual-energy CT-based material extraction for tissue segmentation in Monte Carlo dose calculations. Phys. Med. Biol. 2008; $53: 2439-56$.

${ }^{[6]}$ Landry G, Granton P V, Reniers B, Ollers MC, Beaulieu L, Wildberger JE, et al. Simulation study on potential accuracy gains from dual energy CT tissue segmentation for low-energy brachytherapy Monte Carlo dose calculations. Phys. Med. Biol. 20II;56:6257-78.

[7] Clarkson R, Lindsay PE, Ansell S, Wilson G, Jelveh S, Hill RP, et al. Characterization of image quality and image-guidance performance of a preclinical microirradiator. Med. Phys. 2011;38:845-56.

${ }^{[8]}$ Granton P V, Verhaegen F. On the use of an analytic source model for dose calculations in precision image-guided small animal radiotherapy. Phys. Med. Biol. 2013;58:3377-95.

${ }^{[9]}$ Ma CM, Coffey CW, DeWerd L a, Liu C, Nath R, Seltzer SM, et al. AAPM protocol for $40-300 \mathrm{kV}$-ray beam dosimetry in radiotherapy and radiobiology. Med. Phys. 200I; $28: 868-93$.

${ }^{[\mathrm{rol}]}$ Feldkamp LA, Davis LC, Kress JW. Practical cone-beam algorithm. J. Opt. Soc. Am. A. I984;I:6I2.

[ri] Schyns LEJR, Almeida IP, van Hoof SJ, Descamps B, Vanhove C, Landry G, et al. Optimizing dual energy cone beam CT protocols for preclinical imaging and radiation research. Br. J. Radiol. 20I6;I-IO.

${ }^{\left[{ }_{2}\right]}$ Saito M. Potential of dual-energy subtraction for converting CT numbers to electron density based on a single linear relationship. Med. Phys. 2012;39:202I-30.

${ }^{\left[{ }_{3}\right]}$ Landry G, Seco J, Gaudreault M, Verhaegen F. Deriving effective atomic numbers from DECT based on a parameterization of the ratio of high and low linear attenuation coefficients. Phys. Med. Biol. 2013;58:685I-66. 
$\left.{ }^{\left[{ }^{4}\right]}\right]$ International Commission on Radiation Units and Measurements Report 44. Tissue Substitutes in Radiation Dosimetry and Measurement. I989.

${ }^{\left[{ }^{5}\right]}$ van Hoof SJ, Granton P V, Verhaegen F. Development and validation of a treatment planning system for small animal radiotherapy: SmART-Plan. Radiother. Oncol. 20I3;I09:36I-6.

${ }^{[16]}$ Walters B, Kawrakow I, Rogers DWO. DOSXYZnrc Users Manual. NRCC Rep. PIRS0794. 20I6;I-I25.

${ }^{\left[{ }_{17}\right]}$ Kawrakow I. EGSnrc: The EGSnrc Code System : Monte Carlo Simulation of Electron and Photon Transport. Man. - Guid. 20I3;200I-I3.

${ }^{[18]}$ Kawrakow I, I. Kawrakow. The EGSnrc Code System: Monte Carlo Simulation of Electron and Photon Transport. Med. Phys. 2013;34:200I-I3.

${ }^{[19]}$ Poludniowski, Evans PM. Calculation of $\mathrm{x}$-ray spectra emerging from an $\mathrm{x}$-ray tube. Part I: Electron penetration characteristics in x-ray targets. Med. Phys. 2007;34:2175-86.

${ }^{[20]}$ Poludniowski G, Landry G, DeBlois F, Evans PM, Verhaegen F. SpekCalc: a program to calculate photon spectra from tungsten anode x-ray tubes. Phys. Med. Biol. 2009;54:N4338.

${ }^{[21]}$ Chow JCL, Leung MKK, Lindsay PE, Jaffray D a. Dosimetric variation due to the photon beam energy in the small-animal irradiation: a Monte Carlo study. Med. Phys. 2010;37:5322-9.

${ }^{[22]}$ Bazalova M, Graves EE. The importance of tissue segmentation for dose calculations for kilovoltage radiation therapy. Med. Phys. 2011;38:3039-49.

${ }^{[23]}$ Granton P V, Dubois L, van Elmpt W, van Hoof SJ, Lieuwes NG, De Ruysscher D, et al. A longitudinal evaluation of partial lung irradiation in mice by using a dedicated imageguided small animal irradiator. Int. J. Radiat. Oncol. Biol. Phys. Elsevier Inc.; 2014;90:696704 .

${ }^{[24]}$ Balvert M, van Hoof SJ, Granton P V, Trani D, den Hertog D, Hoffmann AL, et al. A framework for inverse planning of beam-on times for $3 \mathrm{D}$ small animal radiotherapy using interactive multi-objective optimisation. Phys. Med. Biol. Institute of Physics Publishing; 2015;60:568I-98.

${ }^{[25]}$ Chow JCL. Depth dose dependence of the mouse bone using kilovoltage photon beams: A Monte Carlo study for small-animal irradiation. Radiat. Phys. Chem. Elsevier; 2010;79:567-74.

${ }^{[26]}$ Landry G, Gaudreault M, van Elmpt W, Wildberger JE, Verhaegen F. Improved dose calculation accuracy for low energy brachytherapy by optimizing dual energy CT imaging protocols for noise reduction using sinogram affirmed iterative reconstruction. Zeitschrift für Medizinische Phys. Elsevier GmbH; 2016;26:75-87. 



\section{CHAPTER 3}

The effect of different image reconstruction techniques on pre-clinical quantitative imaging and dual-energy CT

Ana Vaniqui*, Lotte EJR Schyns*, Isabel P Almeida, Brent van der Heyden, Mark Podesta and Frank Verhaegen *contributed equally

British Journal of Radiology

November 2018, 92(1095), 20180447 


\section{Abstract}

Objectives: To analyse the effect of different image reconstruction techniques on image quality and dual-energy CT (DECT) imaging metrics.

Methods: A software platform for pre-clinical Cone Beam CT (CBCT) x-ray image reconstruction was built using the opensource reconstruction toolkit (RTK). Pre-processed projections were reconstructed with filtered back-projection and iterative algorithms. Imaging metrics were quantitatively assessed, using a quality assurance phantom, and DECT analysis was performed to determine the influence of each reconstruction technique on the relative electron density $\left(\rho_{\mathrm{c}}\right)$ and effective atomic number $\left(Z_{\text {eff }}\right)$ values.

Results: IR had favourable results for the DECT analysis: a significantly smaller spread for each material in the $\rho_{\mathrm{e}}-Z_{\text {eff }}$ space and lower $Z_{\text {eff }}$ and $\rho_{\mathrm{e}}$ residuals (on average $24 \%$ and $25 \%$ lower, respectively). In terms of image quality assurance, the techniques FDK, Iterative FDK and SART provided acceptable results. The three reconstruction methods showed similar geometric accuracy, uniformity and CT number results. The technique SART had a contrast-to-noise ratio up to $76 \%$ higher for solid water and twice as high for Teflon, but resolution was up to $28 \%$ lower when compared to the other two techniques.

Conclusions: Advanced image reconstruction can be beneficial, but the benefit is small, and calculation times may be unacceptable with current technology. The use of targeted and down-scaled reconstruction grids, larger, yet practicable, pixel sizes and GPU are recommended.

Advances in knowledge: An iterative CBCT reconstruction platform was build using RTK. 


\subsection{Introduction}

Pre-clinical Cone Beam CT (CBCT) $\mathrm{x}$-ray image reconstruction is typically described by the Feldkamp, Davis, and Kress ${ }^{[\mathrm{I}]}$ (FDK) algorithm to solve the inverse Radon transform through filtered back-projection (FBP). While the FDK algorithm produces usually acceptable images, it copes poorly with noise. Iterative reconstruction (IR) algorithms use multiple repetitions in which a current solution converges towards a better solution. Subsequent to projection acquisition, a forward projection creates artificial data, which is compared with measured projections to compute a correction term. In case of discrepancy, the first image estimate is updated based on the characteristics of the underlying algorithm. Image and projection data correction are repeated until a condition predefined by the algorithm is satisfied and the final image is generated ${ }^{[2]}$.

IR algorithms may allow considerable dose reduction due to a more precise modelling of the acquisition process, which can reduce image noise. They avoid introducing new artefacts due to approximations and are suited for dealing with missing data or irregular sampling. It has been suggested ${ }^{|3-5|}$ that IR algorithms could provide superior material segmentation when performing dual-energy CT (DECT). The main drawback of IR is the increased computational cost of the iteration cycle.

This work presents a software platform for pre-clinical CBCT image reconstruction, built using the open-source reconstruction toolkit (RTK ${ }^{[6]}$, which comprises FBP and four IR algorithms. Imaging parameters were quantitatively assessed and a DECT analysis was performed to determine the influence of each reconstruction technique on the image quality.

\subsection{Materials and methods}

\subsection{I (Pre) Image acquisition}

Image projections for this study were acquired using the CBCT electronic portal imaging device (EPID) integrated in the small animal irradiator $\mathrm{X}-\mathrm{RAD}{ }_{225} \mathrm{Cx}$ (Precision X-Ray, North Branford, CT), illustrated in Figure 3.I(f). The irradiator consists of a dual-focus $\mathrm{X}$-ray tube with a maximum tube potential of $225 \mathrm{kV}$ (225 Cx, Comet, Switzerland) and a $20^{\circ}$ angled tungsten stationary target. Using the small focal spot (I.o mm), the X-ray tube acts as photon source for CT imaging. Photons are filtered through a $0.8 \mathrm{~mm}$ beryllium exit window and an additional $2.0 \mathrm{~mm}$ aluminium filter. The source to isocentre distance was fixed at $303.6 \mathrm{~mm}$. The EPID (XRD- 
o820-AN3-ES, Perkin Elmer ${ }^{\circledR}$, Waltham, MA) has Io24 x I024 active pixels in an area of $20 \mathrm{~cm} \mathrm{x} 20 \mathrm{~cm}$.

The pre-processing of the raw data is described in Appendix A and Figure 3.I(a-e). Table 3.I shows scan pre-sets for this study. The exposures were chosen to yield an average dose of 30 cGy for each energy in a DECT scenario. The absorbed dose to water at a solid water phantom surface was verified using a TN3oor2 Farmer ionization chamber (PTW, Freiburg, Germany) according to the AAPM TG-6I protocol for $40-300 k \mathrm{~V}$-ray beam dosimetry (in-air calibration method) ${ }^{[7]}$.

\begin{tabular}{|c|c|c|c|c|c|}
\hline \multicolumn{6}{|c|}{ Image acquisition pre-sets } \\
\hline $\begin{array}{l}\text { Energy } \\
{[k \vee p]}\end{array}$ & $\begin{array}{c}\text { Current } \\
{[\mathrm{mA}]}\end{array}$ & $\begin{array}{c}\text { Frame Rate } \\
\text { [fps] }\end{array}$ & $\begin{array}{c}\text { Rotational Speed } \\
{[\mathrm{fps}]}\end{array}$ & $\begin{array}{l}\text { Image Mode } \\
\text { Gain }\end{array}$ & $\begin{array}{c}\text { Exposure } \\
\text { [mAs] }\end{array}$ \\
\hline 40 & 9,26 & 7,5 & 0,50 & Mid & IIII, 2 \\
\hline 50 & 5,59 & $\mathrm{IO}, \mathrm{O}$ & 0,50 & Mid & 670,8 \\
\hline 60 & 3,18 & IO,O & $\mathrm{o}, 40$ & Mid & 477 \\
\hline 70 & $\mathrm{I}, 54$ & 7,5 & 0,25 & Mid & 369,8 \\
\hline 80 & 2,5 & 5,0 & 0,50 & Low & 300 \\
\hline 90 & 2,08 & 5,0 & 0,50 & Low & 249,6 \\
\hline 100 & $\mathrm{I}, 78$ & 5,0 & 0,50 & Low & 213,6 \\
\hline ІІо & $\mathrm{I}, 54$ & 5,0 & 0,50 & Low & I 84,8 \\
\hline $\mathrm{I} 2 \mathrm{O}$ & I, 36 & 5,0 & 0,50 & Low & 163,2 \\
\hline
\end{tabular}

Table 3.I - Cone beam CT scan pre-sets for energies in the range of 40 to I20 $\mathrm{kVp}$. For dual-energy CT protocols, the mean dose is 30 cGy for each energy.

\subsubsection{Image Reconstruction software}

To compare and characterize both methods, FBP and IR, software was developed using the open-source RTK toolkit ${ }^{[6,8]}$ in the language $\mathrm{C}++$. The software currently comprises five of RTK's reconstruction algorithms, namely, FDK, and four IR methods: iterative FDK ${ }^{[9,}$,o], simultaneous algebraic reconstruction technique $(\mathrm{SART})^{[\mathrm{Ir}, \mathrm{r2}]}$, simultaneous iterative reconstruction technique (SIRT) $)^{\left[{ }_{3}\right]}$ and Conjugate Gradient $(\mathrm{CG})^{\left[\left[_{4}\right]\right.}$. Appendix B has a brief description of the IR techniques. Table 3.2 shows the possible reconstruction scenarios and filters included in the software. 
Reconstruction Algorithm

Characteristics

Reconstruction Size

Reconstruction Spacing

Output format

Cupping correction

Specimen Position

Back-projection filter

Forward projection Filter

Window Type
FDK, Iterative FDK, SART, SIRT, CG

Number of iterations, convergence factor, enforce positivity

Any, used: 1024 pixels x 1024 pixels x 1024 pixels

Any, used: $0.1034 \mathrm{~mm} \times 0.1034 \mathrm{~mm} \times 0.1034 \mathrm{~mm}$

DICOM, MHA, MHD

Feet First: Prone (FFP), Supine (FFS), Decubitus Right (FFDR), Decubitus Left (FFDL)

Head First: Prone (HFP), Supine (HFS), Decubitus Right (HFDR), Decubitus Left (HFDL)

None, (Cuda) Voxel-based back-projection, Joseph, normalized Joseph, Cuda ray cast

None, Joseph, Ray Cast Interpolator, Cuda Ray Cast

None, Hann, Cosine, Hamming, RamLak or Shepplogan (cut-off frequency)

Table 3.2 - Possible reconstruction scenarios using the software developed with the RTK toolkit. For each reconstruction, a selection of the parameters listed here was supplied to the software. All algorithms were investigated, with or without cupping correction, with different numbers of iterations. On the right column, the terms in italic represent the parameters used in this study.

A cupping correction ${ }^{[55]}$ was included in the software. As cupping artefacts are induced by nonlinearities in the projection data, a polynomial precorrection was applied to the attenuation data for linearization. In the image domain, for each energy and reconstruction algorithm, polynomial coefficients were determined once using a homogeneous water cylinder (with a diameter of $\phi=4 \mathrm{~cm}$ ) as a calibration scan. A series of basis images were fitted to a template image, obtained from the pre-processed calibration scan, to generate the coefficients. Raw data was passed through the polynomial and pre-corrected. 
Figure 3.I shows the image acquisition/reconstruction workflow. After initial corrections (Figure 3.I (a-e)) the object was scanned (Figure 3.I(f)). If no cupping correction was applied, the cycle would follow the upper part of the diagram (Figure 3.I $(\mathrm{g})$ ). A single reconstruction of the object was generated with the reconstruction software (RS), followed by a MATLAB ${ }^{\circledR}$ v. R2oifb (The MathWorks Inc., Natick, MA) routine to determine air and water grey levels (rescale function), and a second reconstruction with corrected levels to yield Hounsfield Units (HU). In case of cupping correction, to determine the coefficient $c_{n}$ of the term $x^{\mathrm{n}}$ of the polynomials ${ }^{[15]},(n+\mathrm{I})$ reconstructions of the water phantom were performed. The coefficients were determined through a MATLAB ${ }^{\circledR}$ script (cupping function), followed by a final reconstruction with the identified polynomial values. No further correction was necessary; this method results in attenuation images which are subsequently converted to $\mathrm{HU}$. For the two cycles, apart from the correction coefficients, all other parameters were maintained for both reconstruction rounds.

Figure 3.I: Image acquisition and reconstruction workflow. Pre-acquisition corrections: (a) defective pixels, (b) dark field, (c) flood field, (d) lateral and (e) longitudinal offset for each gantry angle (flexmap). (f) Cone beam CT acquisition, X-ray tube and the flat panel rotate $360^{\circ}$ around the object and generate the pre-processed projection data. (g) Reconstruction cycle, with or without cupping correction. The boxes with RS, Rescale and Cupping function indicate where the reconstruction software (RS) and where MATLAB routines were used, respectively. The boxes with Phantom or Water cylinder illustrate the reconstructed object at that step. The inner image at the centre indicates the reconstruction algorithms. They could be either FBP or IR. IR methods follow the cycle of panel h.

${ }^{\mathrm{I}} \mathrm{HU}=1000 \cdot \frac{\mu-\mu_{\text {water }}}{\mu_{\text {water }}-\mu_{\text {air }}}$ where $\mu_{\text {water }}$ and $\mu_{\text {air }}$ are respectively the linear attenuation coefficients of the medium, water and air. 


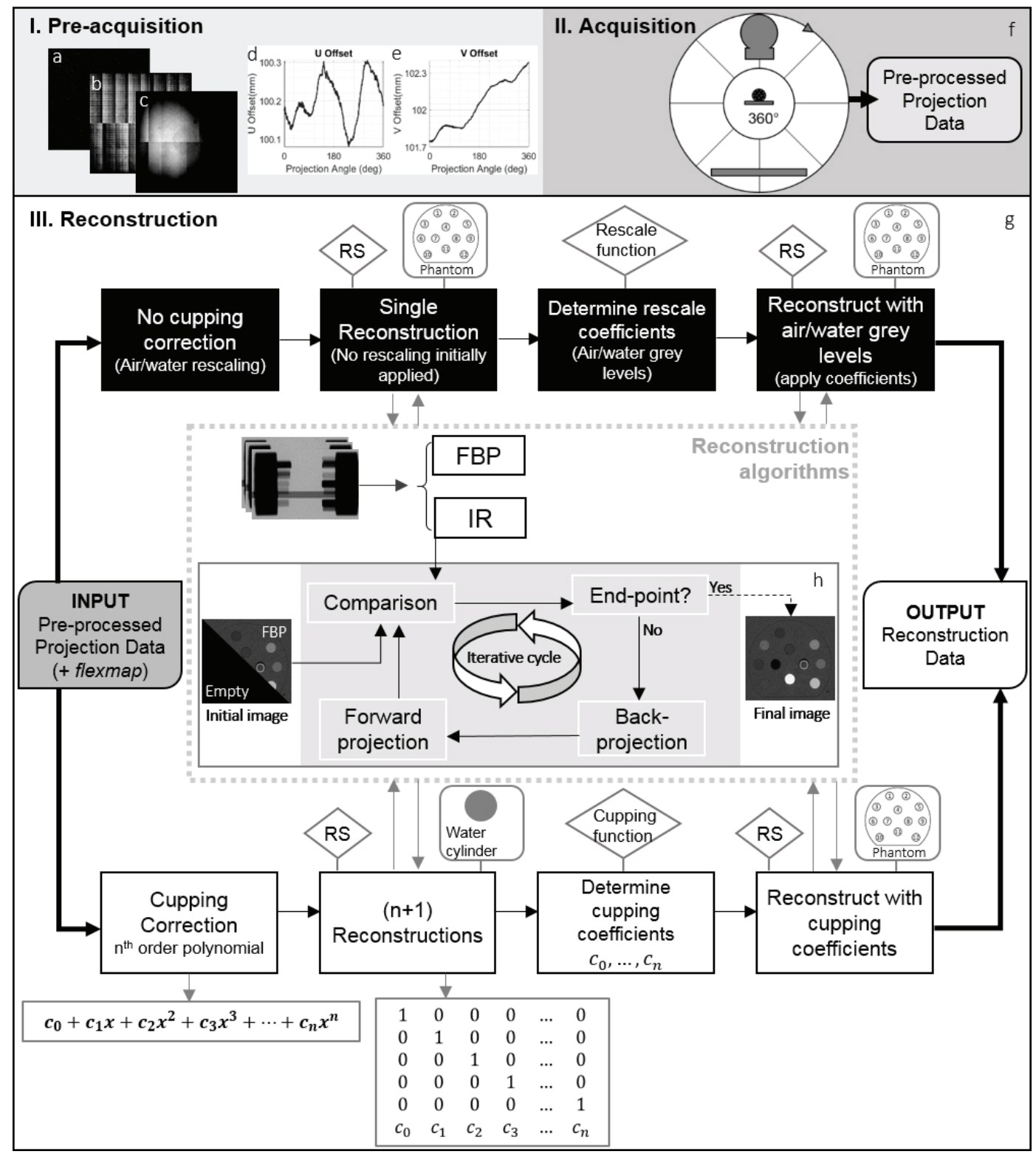




\subsubsection{Image Quality Assurance}

A quantitative image analysis was performed to verify the impact of the different reconstruction techniques using the mCTP-6Io phantom (Shelley Medical Imaging Technologies, Toronto, Canada) ${ }^{[\mathrm{r} 6]}$, Figures 3.2(a-e). It is a single device with modular plates, each designed to assess one aspect of image quality, such as CT number, image uniformity, geometric accuracy, spatial resolution and noise. The plates used in this study are enclosed in a polycarbonate cylindrical housing of $8.4 \mathrm{~cm}$ length, $7.0 \mathrm{~cm}$ diameter and 4.8 $\mathrm{mm}$ thickness. The analysis following previously published methods ${ }^{[16-18]}$ is briefly described.

Figure 3.2(a) shows the CT evaluation plate with volumes of interest (VOIs) of Ioxiox4 pixels. The VOIs shaded in red were used to calculate the mean CT numbers of seven tissue-equivalent embedded materials: SB3, solid water, Lucite, muscle, adipose, high-density polyethylene and Teflon. The material Microfil, a silicone-based vascular contrast agent, was not analysed due to its non-uniformity. The green shaded VOIs were used to calculate the contrast to noise ratio (CNR), according to equation I, where $\bar{I}_{\text {tissue }}$ is the mean CT number of a tissue (red VOI), $\overline{\mathrm{I}}_{\text {background }}$ is mean CT number of the background (adjacent green VOI) and $\sigma_{\text {tissue }}$ and $\sigma_{\text {background }}$ are the standard deviations of $\bar{I}_{\text {tissue }}$ and $\overline{\mathrm{I}}_{\text {background. }}$

$$
\text { CNR }=\frac{\bar{I}_{\text {tissue }}-\bar{I}_{\text {Background }}}{\sqrt{\sigma_{\text {tissue }}^{2}+\sigma_{\text {background }}^{2}}}
$$

Image uniformity was evaluated by average signal difference (centre to peripheral) of five circular regions of interest (ROIs) with diameters $(\phi)$ of Ioo voxels at a uniform polycarbonate plate positioned near the centre of the phantom (Figure 3.2(b)). From the geometric accuracy plate (Figure $3.2(\mathrm{c}))$ the voxel size was determined by dividing the known physical distance between five neighbouring tungsten-carbide beads by the measured distance between the centroid of the respective beads in voxels. The modulation transfer function (MTF) was obtained using the resolution coil plate (Figure 3.2(d)), according to equation $2^{[19]}$

$$
\operatorname{MTF}(f)=\frac{\pi \sqrt{2}}{4} \cdot \frac{M(f)}{\mathrm{M}_{0}}
$$


where $\mathrm{M}(f)$ is the standard deviation of pixel values from ROIs of $20 \times 20$ voxels within each coil, corrected for noise and Mo is half of the absolute difference between the aluminium and Mylar in HU. The coils are made of alternating aluminium and Mylar sheets with 500, 300, 200, and $150 \mu \mathrm{m}$ thicknesses, corresponding to spatial resolution of I, I.67, 2.5, and 3.3 line pairs per $\mathrm{mm}\left(\mathrm{l} \cdot \mathrm{m} \mathrm{m}^{-1}\right)$. MTF was also determined using the slanted edge plate $^{[20]}$. A profile through the slanted edge (Figure 3.2e) defines the edgespread function (ESF), when differentiated yields the line spread function (LSF) and the Fourier transform of the LSF determines the MTF ${ }^{[19]}$.

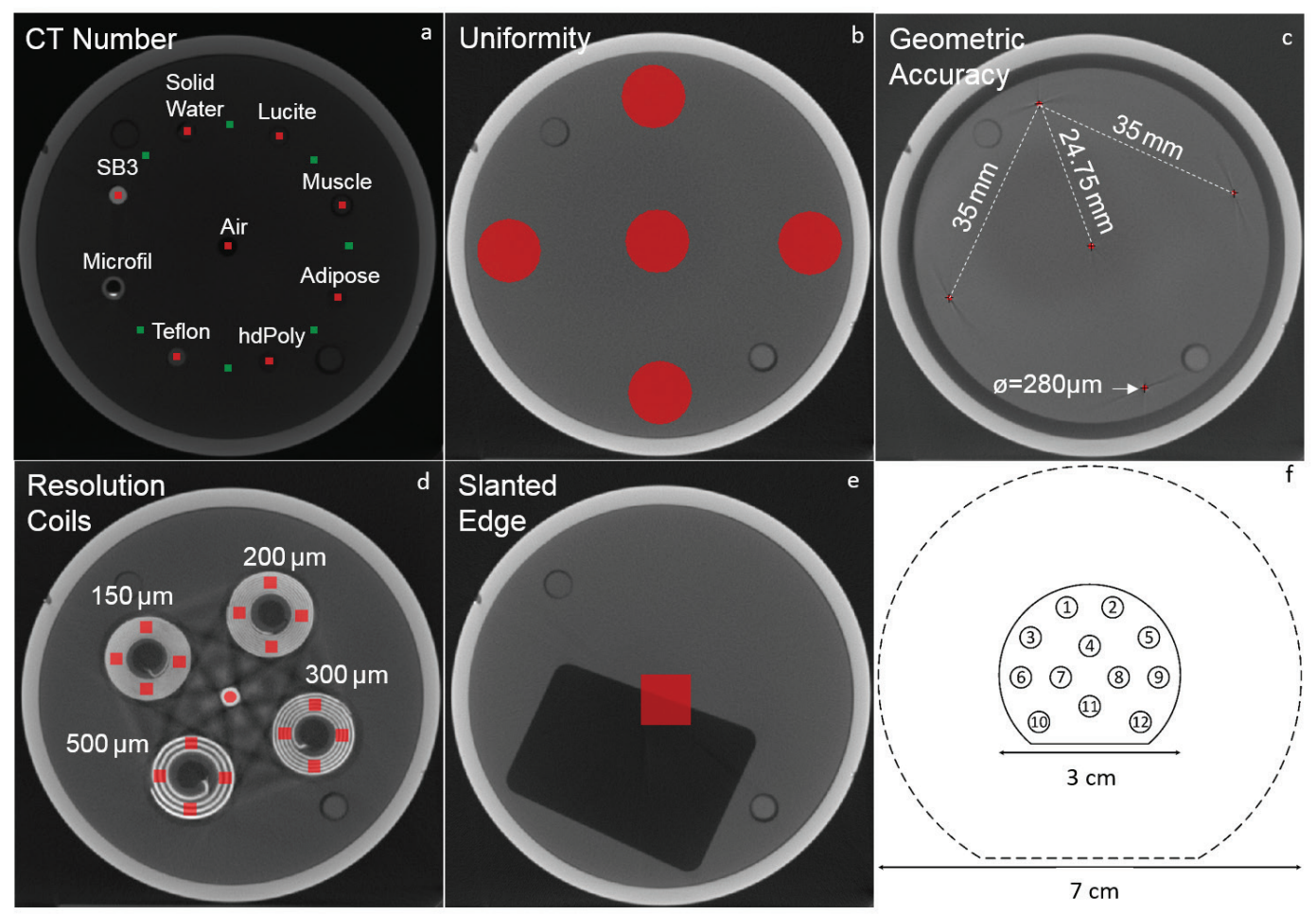

Figure 3.2 - Phantoms used in this study. (a-e) Different plates of the mCTP-6ro quality assurance phantom: (a) CT Number, (b) uniformity, (c) geometric accuracy, (d) resolution coils and (e) slanted edge. The regions of interest used for the image analysis are shaded in red and green. (f) Schematic drawing of the calibration and validation phantoms used for the dual-energy CT analysis. The dashed line represents the $7 \mathrm{~cm}$ diameter phantom, which is composed by an outer ring encompassing the $3 \mathrm{~cm}$ phantom, represented by the solid line. Both rings are made of the same bulk material (solid water). 


\subsubsection{Dual-energy CT}

Two pairs of geometrically identical cylindrical phantoms (SmART Scientific Solutions BV, Maastricht, the Netherlands), Figure 3.2(f), were used for DECT analysis. The phantoms were 3.0 or $7.0 \mathrm{~cm}$ diameter, which corresponds to the cross-section of small rodents, mice to large rats. All phantoms were I cm in length. They were composed of a solid water bulk and twelve cylindrical inserts of $0.35 \mathrm{~cm}$ diameter and $.60 \mathrm{~cm}$ length. Table 3.3 lists the composition of the inserts, which mimic human tissue attenuation properties.

CT numbers were extracted from ROIs of the inserts in the four central slices of a high energy $\left(H U_{\mathrm{H}}\right)$ and a low energy $\left(H U_{\mathrm{L}}\right)$ scan of the DECT phantoms. The procedure described by Schyns et al. ${ }^{[21]}$ to determine the relative electron density $\left(\rho_{\mathrm{e}}^{2}\right)$, using Saito's ${ }^{[22]}$ approach, and to extract the effective atomic number $\left(Z_{\text {eff }}{ }^{[23]}\right.$, following the method proposed by Landry et al. ${ }^{[24]}$ was adopted. The reference values of $Z_{\text {eff }}$ and $\rho_{\mathrm{e}}$, calculated from the compositions and mass densities provided by the manufacturer, and the calculated values from the DECT images were used to assign a tissue composition to each voxel.

\subsection{Results}

\subsection{Reconstruction time}

To generate optimal and reproducible results for image analysis, reconstruction matrices of IO24 X IO24 X IO24 pixels $^{3}$ were used. This matrix size was chosen to avoid reconstruction artefacts and inaccuracies smaller grids could cause, and to fully reconstruct the mCTP-6ro phantom and the pair of $7 \mathrm{~cm}$ DECT phantoms, which were positioned parallel to each other with a gap in between. The small pixel size of (o.ro34 mm)3 was used as it is typically the minimum size at our institution and as the resolution of the scanner was being tested. Figure 3.3a shows that this choice yielded long reconstruction times. The techniques SIRT and CG require a higher

\footnotetext{
$\rho_{\mathrm{e}}=\frac{\left(\frac{N_{\mathrm{A}} \rho Z}{A}\right)}{\left(\frac{N_{\mathrm{A}} \rho_{\mathrm{w}} Z_{\mathrm{w}}}{A_{\mathrm{w}}}\right)}$

, where $\mathrm{N}_{\mathrm{A}}$ is the Avogadro's number, $\rho, Z$ and $A$ are the mass density, atomic number, and atomic mass of a material, while the subscript w indicates water.
}

${ }_{3} Z_{\text {eff }}=\left(\sum_{\mathrm{i}} \mathrm{w}_{\mathrm{i}} Z_{\mathrm{i}}^{\beta}\right)^{\frac{1}{\beta}}$,Wi is the weight fraction of element $\mathrm{i}$ with atomic number and $\beta=3 \cdot 3 \mathrm{I}$ 


\begin{tabular}{lccccccccccc}
\hline $\mathrm{n}^{\circ}$ & material & $\begin{array}{c}\rho \\
{\left[\mathrm{g} / \mathrm{cm}^{3}\right]}\end{array}$ & $\rho_{\mathrm{e}}$ & $Z_{\text {eff }}$ & $\mathrm{H}$ & & $\mathrm{C}$ & $\mathrm{N}$ & & & \\
\end{tabular}

\section{Calibration}

\section{Phantom}

\begin{tabular}{|c|c|c|c|c|c|c|c|c|}
\hline I AP6 (Adipose) & 0.947 & 0.928 & $6.2 \mathrm{IO}$ & 9.06 & 72.30 & 2.25 & 16.27 & $\mathrm{~F}(\mathrm{o.I3})$ \\
\hline 2 Solid Water & 1.022 & 0.992 & 7.735 & 8.00 & 67.30 & 2.39 & 19.87 & $\mathrm{Cl}(\mathrm{O} . \mathrm{I} 4), \mathrm{Ca}(2.3 \mathrm{I})$ \\
\hline 3 IB3 (Inner Bone) & I.I34 & I.086 & I0.4I 8 & 6.67 & 55.64 & $\begin{array}{l}1.96 \\
1.69\end{array}$ & $23.5^{2}$ & $\begin{array}{l}\mathrm{P}(3.23), \mathrm{Cl}(\mathrm{o} . \mathrm{II}) \\
\mathrm{Ca}(8.86)\end{array}$ \\
\hline $4 \mathrm{SR}_{2}$ (Brain) & I.05I & I.047 & 6.090 & 10.83 & 72.54 & 2.12 & I4.86 & $\mathrm{Cl}(0.08)$ \\
\hline $5 \mathrm{CB}_{2-3}-30 \% \mathrm{CaCO}_{3}$ & I.33I & 1.276 & 10.898 & 6.68 & $53 \cdot 48$ & 2.33 & $25.6 \mathrm{I}$ & Cl(o.II), Ca(I2.0I) \\
\hline 6 BRI2 (Breast) & 0.980 & 0.956 & $6.93 \mathrm{I}$ & 8.59 & $7 \mathrm{O} . \mathrm{II}$ & 75.47 & 17.90 & $\mathrm{Cl}(0 . \mathrm{I} 3), \mathrm{Ca}(0.95)$ \\
\hline 7 Air & O.OOI & O.OOI & 7.714 & & & & 23.20 & $\operatorname{Ar}(\mathrm{I} .28)$ \\
\hline 8 Water & I.OOO & I.OOO & $7 \cdot 477$ & II.2O & & I. 98 & 88.80 & \\
\hline 9 Bzoo (Bone Mineral) & I.I $5^{2}$ & I.IO3 & 10.423 & 6.65 & $55 \cdot 5^{2}$ & $\begin{array}{l}2.47 \\
\text { І. } 84\end{array}$ & 23.64 & $\begin{array}{l}\mathrm{P}(3.24), \mathrm{Cl}(\mathrm{o} . \mathrm{II}) \\
\mathrm{Ca}(8.87)\end{array}$ \\
\hline Io LVI (Liver) & I.096 & I.064 & $7 \cdot 736$ & 8.06 & 67.01 & I. $5^{2}$ & 2O.OI & $\mathrm{Cl}(0 . \mathrm{I} 4), \mathrm{Ca}(2.3 \mathrm{I})$ \\
\hline II SB3 (Cortical Bone) & I. 822 & I.695 & I3.638 & $3.4 \mathrm{I}$ & $3 \mathrm{I} .4 \mathrm{I}$ & & 36.50 & $\mathrm{Cl}(0.04), \mathrm{Ca}(26.8 \mathrm{I})$ \\
\hline $\mathrm{I}_{2} \mathrm{CB}_{2}-5 \mathrm{O} \% \mathrm{CaCO}_{3}$ & I. 559 & I.469 & I2.538 & 4.77 & $4 \mathrm{I} .63$ & & 32.00 & $\mathrm{Cl}(0.08), \mathrm{Ca}(2 \mathrm{O} .02)$ \\
\hline
\end{tabular}

\section{Validation}

\section{Phantom}

I BRi2 (Breast)
2 Teflon
3 Lucite
4 Air
5 PMMA
6 Paraffin Wax
7 Water
8 CIRS Muscle
9 Air
Io Air
II Adipose
I2 Bone

I BRi2 (Breast)

2 Teflon

0.980

2.153

I.I 80

O.OOI

I.I9O

0.930

I.OOO

I.062

O.OOI

o.OOI

0.967

I. 600

I. 860

6.931

$8.46 \mathrm{I}$

8.59

7 O.II

24.00

$2.33 \quad 17.90$

$\mathrm{Cl}(\mathrm{o.13}), \mathrm{Ca}(0.95)$

I.I46

6.529

8.05

59.98

7.714

.56

6.529

5.483

0.959

I.OOO

7.477

7.588

I.04

7.714

0.001

7.714

0.956

I. 507
6.439

II. 895
$8.05 \quad 59.98$

I $4.90 \quad 85 . \mathrm{IO}$

II. 20

9.IO

$$
69.70
$$

I0.00

4.83
(1)

\section{$75 \cdot 47 \quad 23.20$}

31.96

F(76.00)

$\operatorname{Ar}(1.28)$

31. 96

88.80

2.IO I6.80 Cl(o.Io), $\mathrm{Ca}(2.20)$

$\begin{array}{llll}75.47 & 23.20 & \operatorname{Ar}(\mathrm{I} .28)\end{array}$

$75.47 \quad 23.20 \quad \operatorname{Ar}($ I.28)

I.80 I6.40 $\mathrm{Cl}(0.2 \mathrm{O}), \mathrm{Ca}(0.3 \mathrm{O})$

$0.97 \quad 35.66$

$\mathrm{Mg}(6.19), \mathrm{Cl}(0.05)$,

$\mathrm{Ca}\left(\mathrm{I}_{5} .24\right)$

Table 3.3 - Reference values of mass density $(\rho)$, relative electron density $\left(\rho_{\mathrm{e}}\right)$, effective atomic number $\left(Z_{\text {eff }}\right)$ and elemental composition of the tissue-substitute materials present in the calibration and validation phantoms. 
number of iterations to converge (Figures $3.3(\mathrm{~b}, \mathrm{c}, \mathrm{h})$ ): after $4 \mathrm{O}$ iterations and 45 computational hours CG presented acceptable results and after 80 hours and Ioo iterations, using a smaller grid, SIRT had not yet converged. They proved to be impractical for further analysis. In the following sections the techniques SART (Figures 3.3 (d,e)), Iterative FDK (Figures $3.3(\mathrm{f}, \mathrm{g})$ ), both with 3 iterations, and FDK (Figure $3.3(\mathrm{i})$ ) are compared. Figure $3 \cdot 3(\mathrm{j})$ shows the reconstruction time of objects with $250 \times 250 \times 200$ pixels $^{3}$ and pixel sizes of $(0.20 \mathrm{~mm})^{3}$ to illustrate a practical pre-clinical scenario, where a smaller volume of interest within a mouse is chosen, with largely reduced reconstruction times.

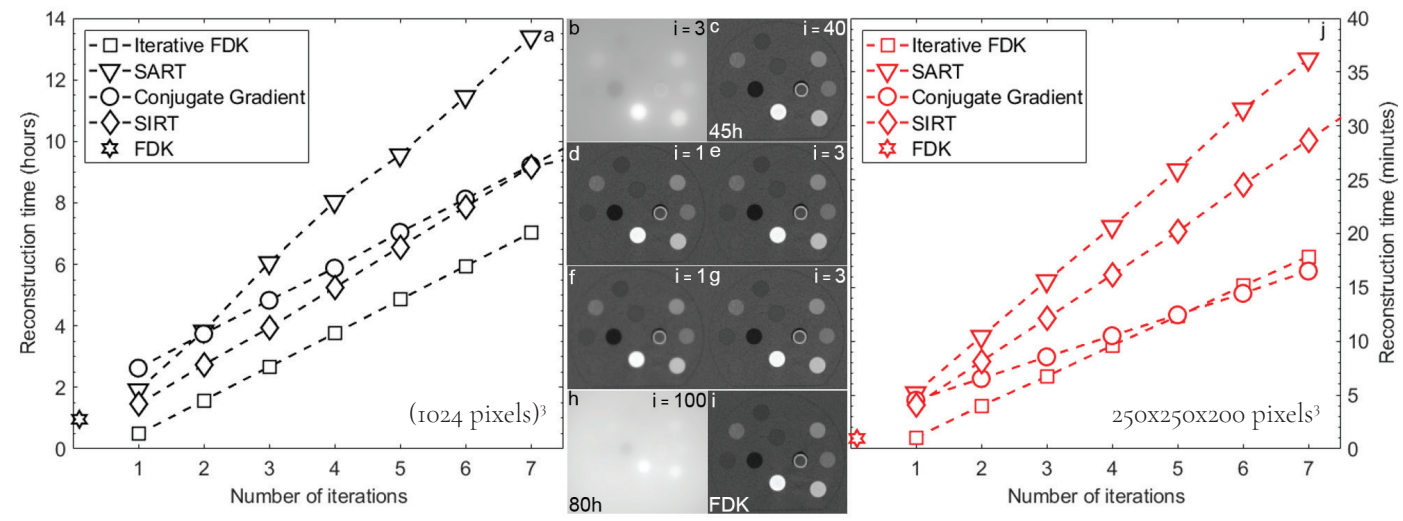

Figure 3.3 - (a) Reconstruction time for each technique versus number of iterations for the gok $\mathrm{Vp}$ images with 602 projections, required by an AMD Opteron ${ }^{\mathrm{TM}}$ Processor 6272, 32 physical cores and I28 GB of RAM. Conjugate Gradient (CG) image after (b) 3 and (c) 40 iterations (45 h). Iterative FDK image after (d) I and (e) 3 iterations. SART image after (f) I and (g) 3 iterations. (h) SIRT image after Ioo iterations for a grid of $\left(5^{\text {I } 2 ~ p i x e l s ~}\right)^{3}$ with pixel size of $(0.2 \mathrm{~mm})^{3}$, which took 8 oh - if the ro24 grid used for the other methods was repeated here the reconstruction times would be even longer. (i) FDK reconstruction. The numbers in the top right corner indicate the number of iterations used in each image. Figures $(b-i)$ were extracted from a central region of the $7 \mathrm{~cm}$ phantom. (b) and (h) present a background different colour because they have not yet converged. (j) Reconstruction times using a smaller grid of 250 pixels x 250 pixels x 200 pixels and voxel size of $(0.2 \mathrm{~mm})^{3}$ to illustrate a scenario closer to a pre-clinical practice workflow, where only a region of interest in the object is selected. Vertical scale is in minutes here, while in panel a it is in hours. 


\subsubsection{Cupping correction}

Different polynomials were tested, ranging from first to fifth order. Figure 3.4 shows phantom HU profiles where a second order polynomial was applied in contrast to the rescaling method. For the corrected cases, the solid water region, or the bulk of the phantom, presents a flatter profile with less pronounced edges. Additionally, the CT values are consistent for bulk, inserts and the outside air. For the rescaling method, air and water grey levels were extracted from inserts within the phantom, which yielded the expected values of -Iooo HU and o HU in the phantom but values lower than -rooo HU outside. The second and third order polynomials yielded the best results in terms of flatter profiles and images with increased visual quality. The method also worked better for energies below rook Vp.

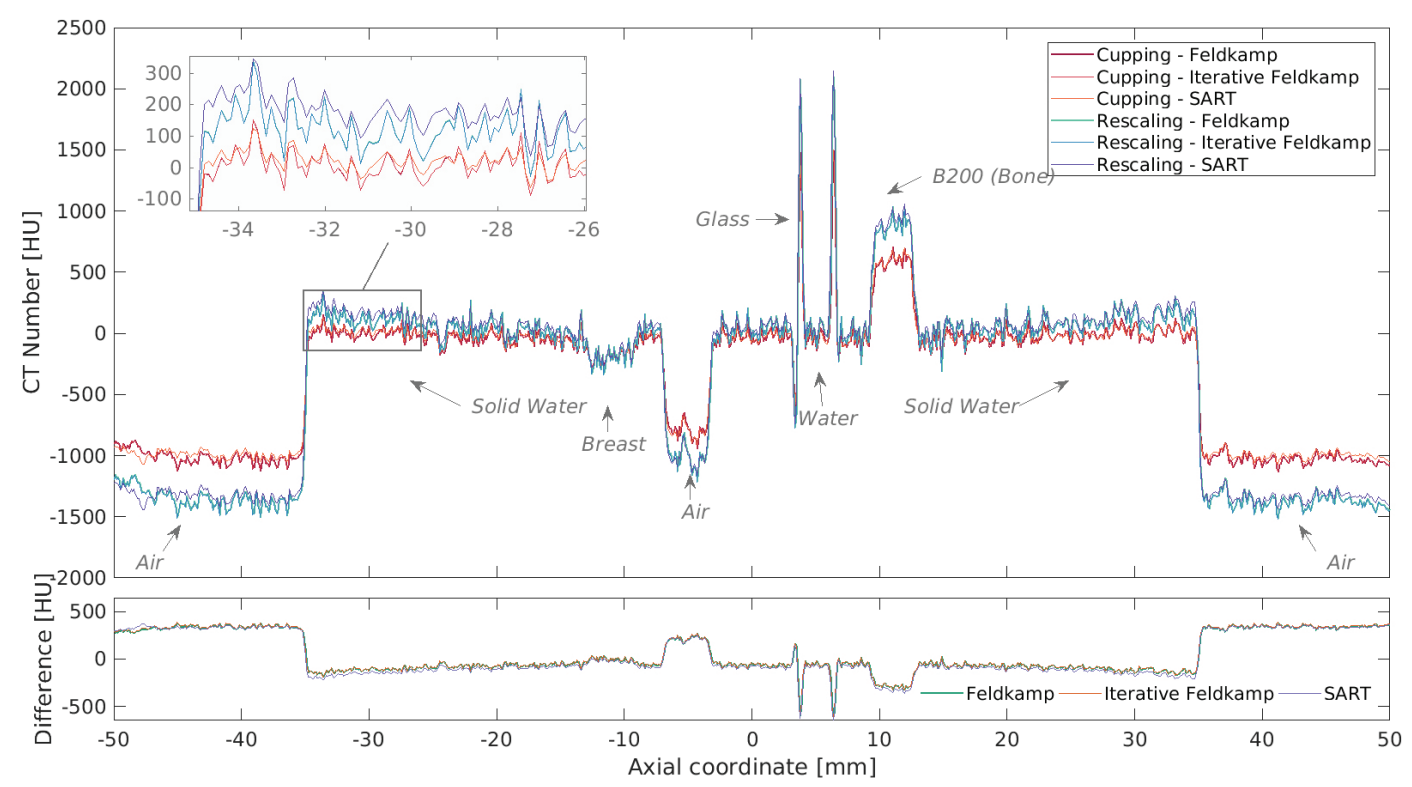

Figure 3.4 - Comparison of phantom HU profiles for different reconstruction methods with and without cupping correction for the $40 \mathrm{kVp}$ scan, where cupping is most evident, of the $7 \mathrm{~cm}$ phantom using a $2^{\text {nd }}$ order polynomial $\left(\mathrm{C}_{2}\right)$. 


\subsubsection{Quantitative image analysis}

Figure 3.5(a) shows the pixel size values derived from the geometric accuracy plate. The values found for the different reconstruction methods and energies were within $0.2 \%$ to $0.3 \%$ in agreement with the value of I03.4 $\mu \mathrm{m}$, used in this study. Figures $3.5(\mathrm{~b}, \mathrm{~d})$ show the CNR for Teflon and solid water. While similar values are obtained for FDK and Iterative FDK, within $-0.05 \%$ to $0.3 \%$, SART yields a higher ratio, up to $76 \%$ higher for solid water and 2.09 times higher for Teflon, which can be explained by the lower levels of noise associated with this technique. Additionally, the panel gain is set to low for energies higher than $70 \mathrm{kVp}$. The uniformity in terms of centre to peripheral average signal difference (Figure 3.5(c)) also yielded similar results for the three methods, with a variation of I2 to I48 HU among the nine investigated energies. CT numbers of seven tissue equivalent materials for energies between 40 to r2okVp (Figure 3.5(e)) showed little variation, with a maximum standard deviation of $45 \mathrm{HU}$ amongst techniques. The values for soft and osseous (or high atomic number) tissues are also within the expected literature values.

Figure 3.5(f) shows that the MTF for both the slanted edge and the resolution coil methods were similar. The highest resolution in terms of equivalent lp. $\mathrm{mm}^{-1}$ at the Io\% MTF $\left(2.5 \mathrm{lp} \cdot \mathrm{mm}^{-1}\right.$ is comparable to $\left.200 \mu \mathrm{m}^{[16]}\right)$, was found for $50 \mathrm{kVp}$, with values of $173.6 \mu \mathrm{m}$ for both FDK and Iterative FDK

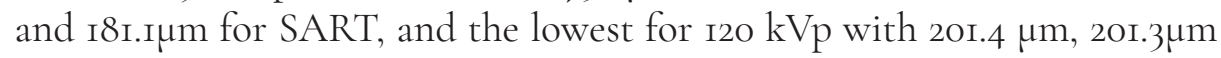
and $234.4 \mu \mathrm{m}$ for FDK, Iterative FDK and SART, respectively. Resolution results for SART were consistently lower when compared to the other two techniques, from $2 \%$ lower, for $60 \mathrm{kVp}$, to $28 \%$, for 1 oo $\mathrm{kVp}$. 

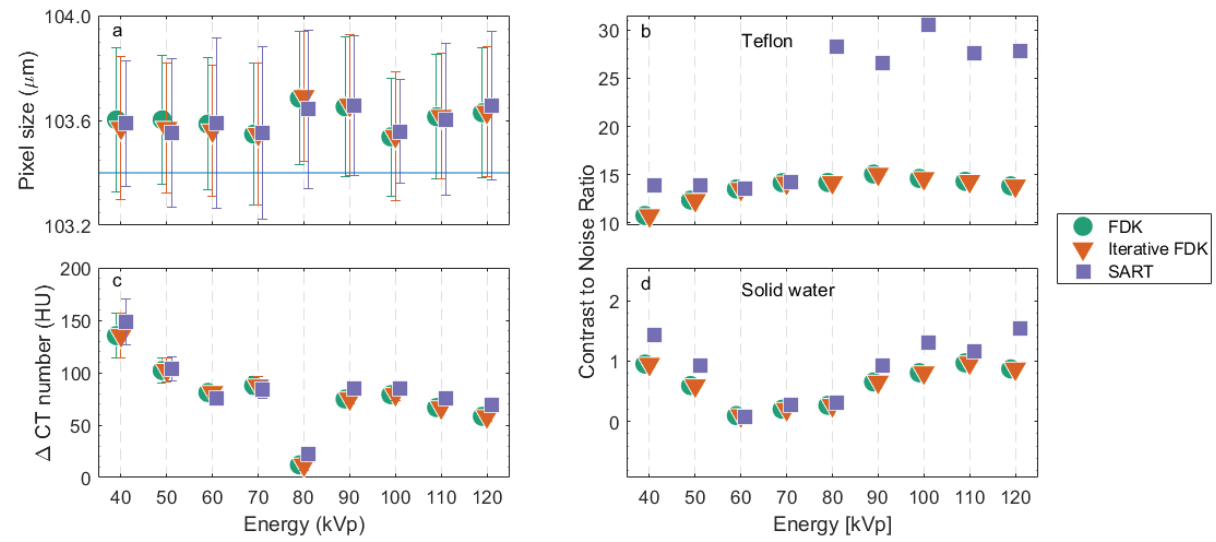

Figure 3.5 - Quantitative image analysis tests with the mCTP-6ro phantom. (a) Geometric accuracy, the blue horizontal line represents the utilized pixel size. (b) CNR for Teflon. (c) Uniformity. (d) CNR for solid water. (e) CT number for tissue equivalent materials for 4 o to r2o $\mathrm{kVp}$, the markers colour shade darkens as the energy increases. (f) MTF using both Slanted Edge (SE) and Resolution Coil (RC) techniques for the $50 \mathrm{kV}$ image. The line indicates the MTF at Io\%. 


\subsubsection{Dual-energy CT analysis}

Figure 3.6 shows the relationship between $Z_{\text {eff }}$ and $\rho_{\mathrm{e}}$ for the materials of the validation phantom. All voxels to which no $Z_{\text {eff }}$ value could be assigned, predominantly located at sharp transitions between air and the solid water bulk, were excluded from the analysis (<о.ог\% in the regions of interest). Ellipses were drawn considering the direction of the eigenvectors of the co-variance matrix. Thus, each distribution confidence ellipse defines the region that contains $95 \%$ of all samples that can be drawn from the underlying Gaussian distribution, with the mean value indicated by the circle, triangle or square. Although the data distribution is similar for FDK and Iterative FDK, the smaller spread of the SART ellipses indicate a reduced image noise effect, expected for an iterative method.

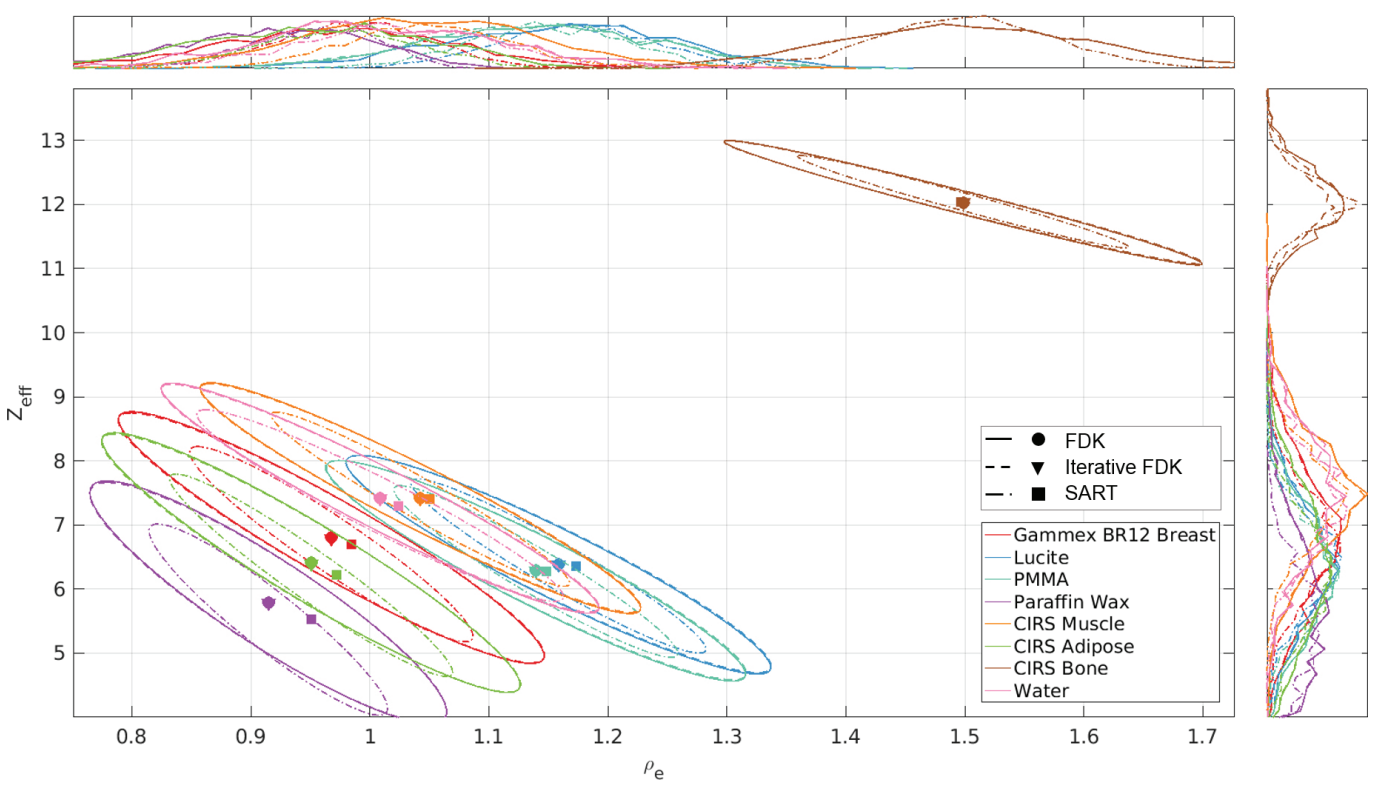

Figure $3.6-\rho_{\mathrm{e}}-Z_{\text {eff }}$ plot for the $7 \mathrm{~cm}$ validation DECT phantom and the energy combination 50 and go $\mathrm{kVp}$. The colours and the lines indicate the tissue-equivalent inserts and reconstruction methods. Histograms of $\rho_{e}$ and $Z_{\text {eff }}$ values are illustrated on top and on the right, respectively. The circles, triangles and squares indicate the mean value of each distribution for FDK, Iterative FDK and SART, respectively. 
Figures $3.7(\mathrm{a}, \mathrm{b})$ show the mean residuals of $\mathrm{Z}_{\mathrm{eff}}$ and $\rho_{\mathrm{e}}$ using the three reconstruction techniques, for a number of energy combinations, where the difference between low and high energy was at least $20 \mathrm{kV}$ apart to avoid large spectral overlap, maximizing the amount of information provided by each energy. For all shown combinations, $Z_{\text {eff }}$ and $\rho_{\mathrm{e}}$ residuals were on average $24 \%$ and $25 \%$ lower, respectively, using SART in comparison with Iterative FDK and FDK - which were within $0.2 \%$ and $0.05 \%$ in agreement with each other in terms of $\mathrm{Z}_{\text {eff }}$ and $\rho_{\mathrm{e}}$ residuals, respectively. Additionally, the mean standard deviations were on average $20 \%$ and $30 \%$ lower with SART in comparison to the other two reconstruction techniques, which once more showed little variation among themselves (average differences of $0.4 \%$ and $0.02 \%$ for $Z_{\text {eff }}$ and $\rho_{\mathrm{e}}$ residuals).
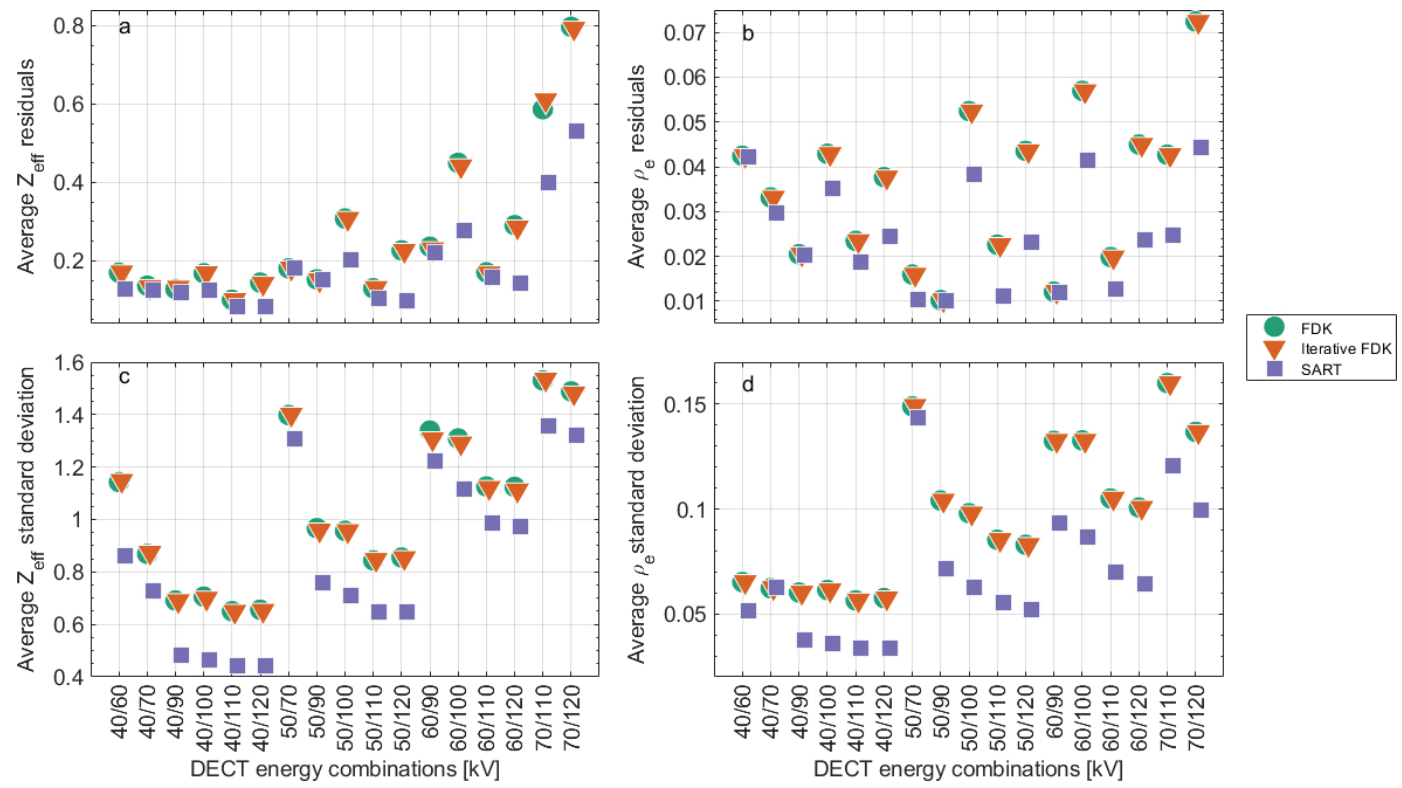

Figure 3.7 - Mean (a) $Z_{\text {eff }}$ and (b) $\rho_{e}$ residuals, and mean (c) $Z_{\text {eff }}$ and (d) $\rho_{e}$ standard deviations for a number of DECT energy combinations. 


\subsection{Discussion}

Iterative reconstructions used in this study were computationally costly and would be impractical for studies with live animals under anaesthesia. The decision to favour the reproducibility, repeating a same grid of $1024 \mathrm{x}$ IO24 X IO24 pixels $^{3}$ for different objects, and using the smallest voxel size of the CBCT scanner of our institution, for a thorough image analysis, affected the speed of the IR. Smaller grids and larger voxel sizes are more typical in pre-clinical practice. In such cases, IR methods were found to be feasible, with reconstruction times under 20 minutes for three iterations (Figure $3 \cdot 3(j)$ ). Additionally, increased computational power, by means of e.g. graphics processing units (GPUs), would highly increase the IR speed. The correction for cupping artefacts included in the software provided accurate CT numbers. The second and third order polynomials yielded the best results, a higher number of coefficients caused further instability and overfitting. The method was more effective for energies below rook $\mathrm{Vp}$, as the cupping effect is more pronounced for lower energies. For IR techniques, reconstructing an object a number of times to derive a polynomial, which will correct a final reconstruction, is a lengthy process. Applications that require increased image quality and larger objects, more prone to beam hardening, could benefit from this approach. For the rescaling method, the coefficients to correct for air and water grey levels are derived from values within the object to account for the beam hardening and other artefacts the object is subjected, as it would be the case of an air pocket within a mouse, for example.

IR provided favourable results for the DECT analysis. SART resulted in a significantly smaller spread for each material in the $\rho_{\mathrm{e}}-Z_{\text {eff }}$ space (Figure 3.6) and the residuals on $Z_{\text {eff }}$ and $\rho_{e}$ were on average $24 \%$ and $25 \%$ lower, in comparison to FDK - with an increasing number of iterations, the residuals decreased further. In terms of image quality, the techniques FDK, Iterative FDK and SART provided acceptable results. Regarding geometric accuracy, the pixel size agreement was within $0.2 \%$ to $0.3 \%$ with the used value of I03.4 $\mu \mathrm{m}$. The three techniques showed similar uniformity and CT number results, with the latter presenting a maximum standard deviation of 45 $\mathrm{HU}$ amongst techniques and soft and osseous tissues within the expected literature values. Although the CNR presented by SART was compelling, a ratio up to $76 \%$ higher for solid water and over twice higher for Teflon, 
the resolution results were up to $28 \%$ lower when compared to the other two techniques. Because of the iterative cycle, IR techniques may produce smoother images, less affected by noise.

Accuracy on the image metrics listed above is crucial for dose calculations, which are based on the CBCT image of small animals. Accuracy of contours of anatomical structures can suffer from reduced resolution, abundance of noise or non-uniformity. Different reconstruction techniques could be used for alternative purposes ${ }^{[25]}$. In a possible irradiation workflow, following the CBCT scan of a mouse, the projections could be quickly reconstructed using the iterative FDK method. Availing from the increased resolution and diminished presence of artefacts, the images could be contoured while the projections are reconstructed using the SART technique. The user could see the result of each iteration, choose the most adequate image, and stop the iterative cycle. The iteratively reconstructed image could be used for dose calculations, which could benefit from the lower levels of noise.

The influence of the scanning parameters and the reconstruction filters could be further investigated. The imaging pre-sets were adopted from previous studies ${ }^{[21]}$, where they were deemed optimal. IR could provide satisfactory results with decreased tube current or scanning time and consequently decreased imaging dose. Lastly, one should be aware of the biological consequences of high CT imaging doses which may even alter the experimental outcome $e^{[17]}$. 


\subsection{Conclusion}

An image reconstruction platform has been built, for modern small animal image guided irradiation platforms using RTK. The software includes backprojection, different iterative algorithms, filters and cupping correction. Pre-processed projections reconstructed with the software showed acceptable results regarding image quality assurance and DECT $Z_{\text {eff }}$ and $\rho_{e}$ residuals. The iterative technique SART showed improved results regarding noise, which may be important for tissue assignment for dose calculations. In a nutshell, advanced image reconstruction can be beneficial, but the benefit is rather small, and calculation times may be unacceptable with current technology.

\subsection{Supplementary Material iA}

\section{Image pre-processing}

Prior to image acquisition, the system was calibrated for dark current, differences in pixel gain, defective pixels and mechanical flex (Figure 3.I(a-e)). For dark current, the electric current that flows through the flat panel when the X-ray tube is inactive, fifty images were collected with the beam switched off and averaged to generate the mean dark image. For the pixel sensitivity, which varies due to fluctuations in gain, associated with the analogue amplifier, fifty beam-on images were collected without any objects in the field. The flood field also corrects for non-flat beam profiles. Subsequent scans were divided by the corrected flood image, the averaged flood field minus the mean dark image, to correct for gain differences. Defective pixels were identified statistically: the variability of each pixel was indicated by the standard deviation of the mean dark and flood images, from which a bad pixel map was generated. To correct for mechanical flex, where CBCT scans are shifted by a lateral and longitudinal offset for each gantry angle due to mechanical sagging, a ball bearing was placed in the irradiator isocentre, imaged from all directions and its location was identified on each image to map the offset, generating a flexmap. 


\subsection{Supplementary Material IB}

Iterative methods: brief description

Iterative FDK consists of filtering out the streaking artefacts from a FDK reconstruction in an iterative deconvolution scheme ${ }^{[9, \text { rol }]}$. SART ${ }^{[1,52]}$, SIRT $^{\left[{ }_{13}\right]}$ and $\mathrm{CG}^{[\mathrm{r}]}$ minimize the same cost function, which consists of a least-squares data-attachment term, i.e. $\|R f-p\|^{2}$, where $f$ is the sought volume, $p$ the projections and $R$ the forward projections, but using different algorithms. SIRT does a gradient descent: as the gradient of the cost function is $2 R^{\top}(R f-$ $p$ ), where $R^{\top}$ is the transpose of $R$ (the back-projection), at each iteration, the algorithm needs one forward and one back projection from all angles, and yields an update of the volume. SART splits the cost function into smaller pieces (individual projections) and alternately minimizes the cost for each piece. One iteration is achieved when one piece has triggered an update of the volume. CG minimizes the same cost function, without splitting it, using the conjugate gradient algorithm, where the step size is calculated analytically at each iteration and the descent direction is a combination of the gradient at the current iteration and the descent direction at the previous iteration (a conjugate direction). 


\subsection{References}

${ }^{[\mathrm{I}]}$ L.A. Feldkamp, L.C. Davis, J.W. Kress, Practical cone-beam algorithm, Journal of the Optical Society of America A, I (I984).

${ }^{[2]}$ M. Beister, D. Kolditz, W.A. Kalender, Iterative reconstruction methods in X-ray CT, Phys Med, 28 (2012) 94-IO8.

${ }^{\left.{ }_{3}\right]}$ A. Vaniqui, L. Schyns, I.P. Almeida, B. van der Heyden, S.J. van Hoof, F. Verhaegen, The impact of dual energy CT imaging on dose calculations for pre-clinical studies, Radiat Oncol, I2 (2017) I8I.

${ }_{44}{ }^{4}$ C. Bouckaert,B. Vandeghinste, C. Vanhove, S. Vandenberghe. Dual energy microCT for small animal bone-iodine decomposition, 20I2 IEEE Nuclear Science Symposium and Medical Imaging Conference Record (NSS/MIC), Anaheim, CA, (2012) 3769-74.

${ }^{\left.{ }_{5}\right]}$ I. Willekens, N. Buls, T. Lahoutte, L. Baeyens, C. Vanhove, V. Caveliers, R. Deklerck, A. Bossuyt, J. de Mey, Evaluation of the radiation dose in micro-CT with optimization of the scan protocol, Contrast Media Mol Imaging, 5 (2OIO) 2OI-207.

${ }^{[6]}$ S. Rit, M. Vila Oliva, S. Brousmiche, R. Labarbe, D. Sarrut, G.C. Sharp, The Reconstruction Toolkit (RTK), an open-source cone-beam CT reconstruction toolkit based on the Insight Toolkit (ITK), Journal of Physics: Conference Series, 489 (2014).

${ }_{77]}$ C.M. Ma, C.W. Coffey, L.A. DeWerd, C. Liu, R. Nath, S.M. Seltzer, J.P. Seuntjens, M. American Association of Physicists in, AAPM protocol for $40-300 \mathrm{kV}$-ray beam dosimetry in radiotherapy and radiobiology, Med Phys, 28 (200I) 868-893.

${ }^{[8]}$ L.L. Geyer, U.J. Schoepf, F.G. Meinel, J.W. Nance, Jr., G. Bastarrika, J.A. Leipsic, N.S. Paul, M. Rengo, A. Laghi, C.N. De Cecco, State of the Art: Iterative CT Reconstruction Techniques, Radiology, 276 (2015) 339-357.

${ }^{[9]}$ C. Mory, V. Auvray, B. Zhang, M. Grass, D. Schafer, S. Rit, F. Peyrin, P. Douek, L. Boussel, Removing streak artifacts from ECG-gated reconstructions using deconvolution, J Xray Sci Technol, 22 (2014) 253-270.

${ }^{[\mathrm{ro}]}$ J. Sunnegårdh, Iterative Filtered Backprojection Methods for Helical Cone-Beam CT, 2009 .

${ }^{[11]}$ R. Gordon, R. Bender, G.T. Herman, Algebraic Reconstruction Techniques (ART) for three-dimensional electron microscopy and X-ray photography, Journal of Theoretical Biology, 29 (I970) 47I-48I.

${ }^{\left[{ }_{2}\right]}$ A.H. Andersen, A.C. Kak, Simultaneous algebraic reconstruction technique (SART): a superior implementation of the art algorithm, Ultrason Imaging, 6 (I984) 8I-94.

${ }_{{ }^{2} 3}$ P. Gilbert, Iterative methods for the three-dimensional reconstruction of an object from projections, Journal of Theoretical Biology, 36 (I972) I05-II7.

${ }^{\left[{ }^{14}\right]}$ R. Fletcher, C.M. Reeves, Function minimization by conjugate gradients, The Computer Journal, 7 (I964) I49-I54. 
${ }^{\left.{ }^{15}\right]}$ M. Kachelriess, K. Sourbelle, W.A. Kalender, Empirical cupping correction: a first-order raw data precorrection for cone-beam computed tomography, Med Phys, 33 (2006) I269I274.

${ }^{[16]}$ L.Y. Du, J. Umoh, H.N. Nikolov, S.I. Pollmann, T.Y. Lee, D.W. Holdsworth, A quality assurance phantom for the performance evaluation of volumetric micro-CT systems, Phys Med Biol, 52 (2007) 7087-7108.

${ }^{\left.{ }_{17}\right]}$ C.D. Johnstone, P. Lindsay, E.E. Graves, E. Wong, J.R. Perez, Y. Poirier, Y. Ben-Bouchta,

T. Kanesalingam, H. Chen, A.E. Rubinstein, K. Sheng, M. Bazalova-Carter, Multiinstitutional MicroCT image comparison of image-guided small animal irradiators, Phys Med Biol, 62 (2017) 5760-5776.

${ }^{[18]}$ S. Sharma, G. Narayanasamy, R. Clarkson, M. Chao, E.G. Moros, X. Zhang, Y. Yan, M. Boerma, N. Paudel, S. Morrill, P. Corry, R.J. Griffin, Study of Image Qualities From 6D Robot-Based CBCT Imaging System of Small Animal Irradiator, Technol Cancer Res Treat, DOI Io.1177/I533034617700558(2017) I533034617700558.

${ }^{\left[{ }_{19}\right]}$ R.T. Droege, R.L. Morin, A practical method to measure the MTF of CT scanners, Med Phys, 9 (1982) 758-760.

${ }^{[20]}$ P.F. Judy, The line spread function and modulation transfer function of a computed tomographic scanner, Med Phys, 3 (1976) 233-236.

${ }^{[21]}$ L.E. Schyns, I.P. Almeida, S.J. van Hoof, B. Descamps, C. Vanhove, G. Landry, P.V. Granton, F. Verhaegen, Optimizing dual energy cone beam CT protocols for preclinical imaging and radiation research, Br J Radiol, 9o (2017) 20160480.

${ }^{\left[{ }_{22}\right]}$ M. Saito, Potential of dual-energy subtraction for converting CT numbers to electron density based on a single linear relationship, Med Phys, 39 (2012) 202I-2030.

${ }^{[23]}$ G. Landry, P.V. Granton, B. Reniers, M.C. Ollers, L. Beaulieu, J.E. Wildberger, F. Verhaegen, Simulation study on potential accuracy gains from dual energy CT tissue segmentation for low-energy brachytherapy Monte Carlo dose calculations, Phys Med Biol, 56 (2011) 6257-6278.

${ }^{\left.{ }^{2} 4\right]}$ G. Landry, J. Seco, M. Gaudreault, F. Verhaegen, Deriving effective atomic numbers from DECT based on a parameterization of the ratio of high and low linear attenuation coefficients, Phys Med Biol, 58 (2013) 6851-6866.

${ }^{{ }^{25}{ }^{2}}$ G. Landry, M. Gaudreault, W. van Elmpt, J.E. Wildberger, F. Verhaegen, Improved dose calculation accuracy for low energy brachytherapy by optimizing dual energy CT imaging protocols for noise reduction using sinogram affirmed iterative reconstruction, Z Med Phys, 26 (2016) 75-87. 



\section{CHAPTER 4}

On the determination of planning target margins due to motion for mice lung tumours using a four-dimensional MOBY phantom

Ana Vaniqui, Brent van der Heyden, Isabel P Almeida, Lotte EJR Schyns, Stefan J van Hoof, Frank Verhaegen

July 2018, 92(1095), 20180445

British Journal of Radiology 


\section{Abstract}

Objectives: This work aims to analyse the effect of respiratory motion on optimal irradiation margins for murine lung tumour models.

Methods: Four-dimensional mathematical phantoms with different lung tumour locations affected by respiratory motion were created. Two extreme breathing curves were adopted and divided into time-points. Each time-point was loaded in a treatment planning system and Monte Carlo (MC) dose calculations were performed for a $360^{\circ}$ arc plan. A time-resolved dose was derived, considering the gantry rotation and the breathing motion. Radiotherapy metrics were derived to assess the final treatment plans. An interpolation function was investigated to reduce calculation cost.

Results: The effect of respiratory motion on the treatment plan quality is strongly dependent on the breathing pattern and the tumour position. Tumours located closer to the diaphragm required a compromise between tumour conformity and healthy tissue damage. A recipe, which considers collimator size, was proposed to derive tumour margins and spare the organs at risk (OARs) by respecting constraints on userdefined metrics.

Conclusions: It is recommended to add a target margin, especially on sites where movement is substantial. A simple to recipe derive tumour margins was developed.

Advances in knowledge: This work is a first step towards a standard planning target volume concept in pre-clinical radiotherapy. 


\subsection{Introduction}

Recent developments in technology and tumour models have produced unprecedented opportunities for pre-clinical translational and radiobiological research. It is currently becoming possible to mimic complex radiotherapy treatments in an experimental pre-clinical setting. For accurate research translation to clinical practice, and ultimate patient benefit, a number of challenges have yet to be overcome. A recent recommendations report ${ }^{[\mathrm{r}]}$ has raised several points that require further study, among which the optimal irradiation margins of tumour models.

The concept of treatment margins is fundamental in radiotherapy, the use of target volumes has long been recommended ${ }^{[2-4]}$ and is a premise for unambiguous prescription, recording and reporting of dose. Although the delineations of the gross and clinical target volumes (GTV and CTV) are purely based on anatomic and biological parameters, the planning target volume (PTV) consists of a geometrical margin to account for variation in size, shape and position relative to the treatment beam. The PTV considers the net effect of all the possible geometrical variations, to ensure that the prescribed dose is absorbed in the CTV ${ }^{[4]}$. A number of clinical studies ${ }^{[5-10]}$ have explored this concept and proposed recipes to quantify the weight of systematic and random errors, or the effect of treatment execution and preparation, on the size of the PTV.

To adopt such concepts, it is important to consider the differences between clinical and pre-clinical irradiation. Small animals, such as mice, are imaged and irradiated under sedation, which promotes physical restraint and depresses respiratory and cardiovascular rate. The cardiac and breathing motions are thus affected and the specimen has to be constantly monitored to avoid, for instance, hypothermia.

In this context, a mathematical 4 -dimensional $\left.{ }_{4} \mathrm{D}\right)$ phantom and two extreme breathing patterns have been explored to analyse the effect of respiratory motion on the dose distribution. A simple recipe, which takes into account the collimator size, is proposed to derive tumour margins and safeguard tolerable radiation levels to the organs at risk (OARs). Furthermore, an interpolation method, based on ${ }_{4} \mathrm{D}$ dose accumulation with rigid progression, to decrease Monte Carlo calculation time is also discussed. 


\subsection{Material and Methods}

\subsection{I $4 \mathrm{D}$ digital Mouse whole-body phantom (MOBY)}

The mathematical mouse whole body phantom, MOBY ${ }^{[\mathrm{rI}]}$, was used. This ${ }_{4} \mathrm{D}$ phantom is capable of generating realistic anatomy models of breathing mice in two physical modes: attenuation or emission. For the former, it simulates a $3 \mathrm{D}$ distribution of attenuation coefficients for a given photon energy, and for the latter, it simulates a $3 \mathrm{D}$ distribution of emission radionuclide activity for the various organs. This study used MOBY in activity mode but instead of radionuclide activities, every organ listed in the activity mode was assigned a human tissue density value from the ICRU Report 44, $1988^{\left[{ }^{[2]}\right]}$. Using human tissue density values is common practice in pre-clinical radiotherapy, due to the absence of small animal tissue density information in the literature ${ }^{\left[{ }^{[3]}\right]}$. As a result of the assignment, each MOBY voxel represents a density value of a specific organ.

A spherical lesion was placed in the left lung, as it is more affected by the heart shape and movement. Different scenarios were generated with two possible lesion diameters and four positions. Figures 4.I (a-d) display the different positions; position I (PI, Figure 4.I(a)) has the tumour located along the diaphragm and is the most affected by motion. Table 4.I enumerates all simulated MOBY scenarios. 

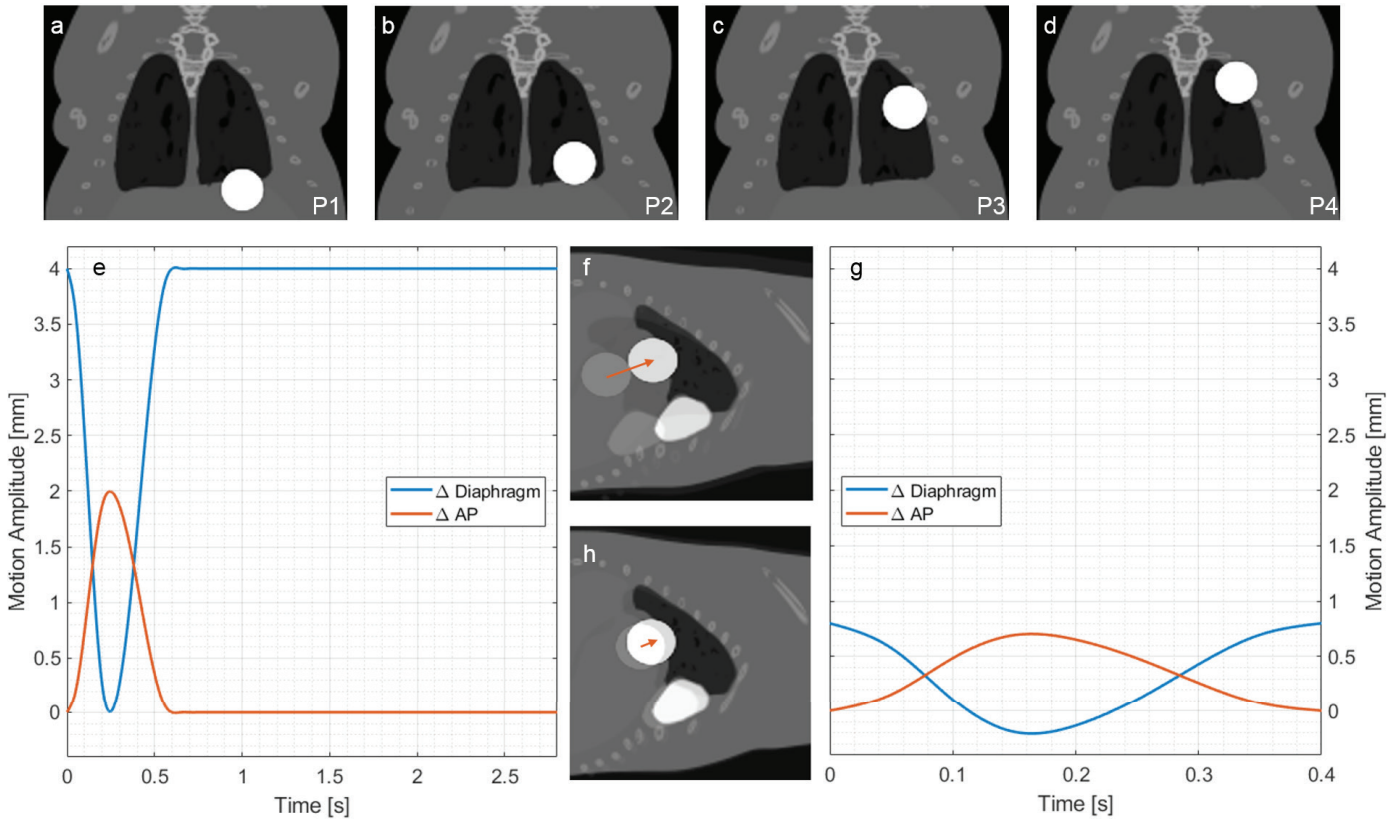

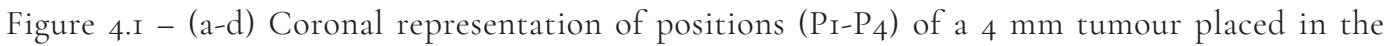
left lung of MOBY phantoms. (e,g) Diaphragm and Anterior-Posterior motion amplitudes as a function of time. (e) Curve obtained by fluoroscopic X-ray imaging of a breathing mouse put under isoflurane anaesthesia. (f) Sagittal representation of the breathing motion of curve re, for a $4 \mathrm{~mm}$ tumour, at $\mathrm{PI}_{\mathrm{I}}$, at two instants: $\mathrm{t}=0.0 \mathrm{~s}$ and $\mathrm{t}=0.3 \mathrm{~s}$. (g) Tidal breathing curve derived from a normal-breathing human, scaled to the dimensions of a mouse. (h) Sagittal representation of the breathing motion of the curve ig, for the same conditions of Figure 4.Ie. The arrow qualitatively shows the tumour displacement in this plane. The white colour of the tumour and heart is merely for visualization purposes, the underlying density values are agreement with soft tissue and heart. 
Moby parameters

\begin{tabular}{|c|c|c|c|}
\hline Organ & & \multicolumn{2}{|l|}{ Left Lung } \\
\hline Tumour diameter & {$[\mathrm{mm}]$} & \multicolumn{2}{|l|}{3 or 4} \\
\hline Tumour position & & \multicolumn{2}{|l|}{$\mathrm{I}, 2,3,4$} \\
\hline Breathing curve & & \multicolumn{2}{|l|}{ Anaesthesia } \\
\hline Maximum diaphragm amplitude & {$[\mathrm{mm}]$} & 4 & I \\
\hline Maximum AP amplitude & {$[\mathrm{mm}]$} & 2 & 0,7 \\
\hline Total Period & {$[s]$} & 2,8 & 0,4 \\
\hline Respiration period & {$[\mathrm{s}]$} & 0,6 & 0,4 \\
\hline Rest period & {$[\mathrm{s}]$} & 2,2 & $\mathrm{o}$ \\
\hline Time interval & {$[\mathrm{s}]$} & \multicolumn{2}{|l|}{0,05} \\
\hline Number of time-points & & \multicolumn{2}{|l|}{56} \\
\hline \multicolumn{4}{|l|}{ SmART-Plan irradiations } \\
\hline Irradiation Plan & & \multicolumn{2}{|l|}{$360^{\circ} \mathrm{Arc}$} \\
\hline Collimator size (C\#) & {$[\mathrm{mm}]$} & \multicolumn{2}{|c|}{$3,4,5,6,7$, or $4,5,6,7,8$} \\
\hline Irradiation dose & {$[\mathrm{Gy}]$} & \multicolumn{2}{|l|}{$8 \mathrm{~Gy}$} \\
\hline Histories & & \multicolumn{2}{|c|}{ Io ${ }^{8} /$ time-point } \\
\hline
\end{tabular}

Table 4.I - Characteristics of MOBY phantoms and SmART-Plan treatment plans.

Figures 4.I (e,g) show the two breathing curves employed in this study, their characteristics are summarized in Table 4.I. The anaesthesia curve, Figure 4.Ie, proposed by van der Heyden et al ${ }^{\left[\mathrm{I}_{4}\right]}$, was derived from fluoroscopy imaging of a mouse under the influence of isoflurane; commonly used in murine in vivo non-invasive imaging and irradiation. As an effect of the anaesthetic, the breathing is deep and slow, the full breathing cycle has a long period, of which nearly $80 \%$ is represented by the rest phase. Although the breathing phase is relatively short, the diaphragm and anterior-posterior (AP) motions are pronounced.

The tidal curve, Figure 4.I $(\mathrm{g})$, proposed by Segars et al ${ }^{[\mathrm{II}]}$, was derived from a normal-breathing human, scaled to the dimensions of a mouse. In comparison to the anaesthesia curve, it has a shorter period and reduced diaphragm and AP motions. The movement is continuous, as there is no rest phase. Figures $4 . \mathrm{I}(\mathrm{f}, \mathrm{g})$ reveal the instants when the motion amplitudes are maximal and minimal, for anaesthesia and tidal, respectively. 
Both curves were converted into $4 \mathrm{D}$ non-uniform rational basis splines (NURBS) for MOBY implementation. NURBS were adopted to model respiratory motion so the breathing curve parameters could be splineinterpolated in time and ${ }_{4} \mathrm{D}$ phantoms generated for any time interval. MOBY software subsequently sampled the NURBS's phantom lung volume in time points.

\subsubsection{Treatment Planning System}

Treatment plans were generated using the small animal treatment planning system (TPS) SmART-Plan ${ }^{[5]}$ (Research Version I.5, PXi, North Branford, CT). As this version does not handle moving geometries, the breathing curves were divided into time-points (or frames) with a period of $5 \mathrm{~ms}$ each. The anaesthesia and tidal curves yielded 56 and 8 time-points, respectively. Each frame was loaded into SmART-Plan as a static voxelized geometry. Automatic structure delineation, based on previously assigned tissue densities and known organ positions, and material allocation for every organ were performed for each frame. Full treatments were automatically generated using MATLAB ${ }^{\circledR}$ v.R2or4a/R2or8a (The MathWorks Inc., Natrick, MA) scripts.

Figure 4.2(a) shows the treatment plan, a dynamic $360^{\circ}$ arc. Table 4.I presents a summary of the TPS simulation protocols. The geometry consisted of 256 x 256 x IOI voxels with $0.2 \times 0.2 \times 0.2 \mathrm{~mm}^{3}$ voxel sizes. The prescription dose to the target volume was set to $8 \mathrm{~Gy}$. This value was achieved considering the normal irradiation conditions for the X-RAD ${ }_{225} \mathrm{Cx}$ irradiator (PXi, North Branford, CT) at our institute: energy of $225 \mathrm{kVp}, \mathrm{I} 3 \mathrm{mAs}$, filter cassette made of copper with nominal thickness of $0.32 \mathrm{~mm}$, source to isocentre distance fixed at $303.6 \mathrm{~mm}$, dose rate of $0.4 \mathrm{I}$ cGy/mAs and different beamon times. The absorbed dose to water is periodically verified at a solid water phantom surface using a TN3oor2 Farmer ionization chamber (PTW, Freiburg, Germany) according to the AAPM TG-6I protocol for 40-300 $\mathrm{kV}$-ray beam dosimetry (in-air calibration method) ${ }^{[\mathrm{r} 6]}$. 


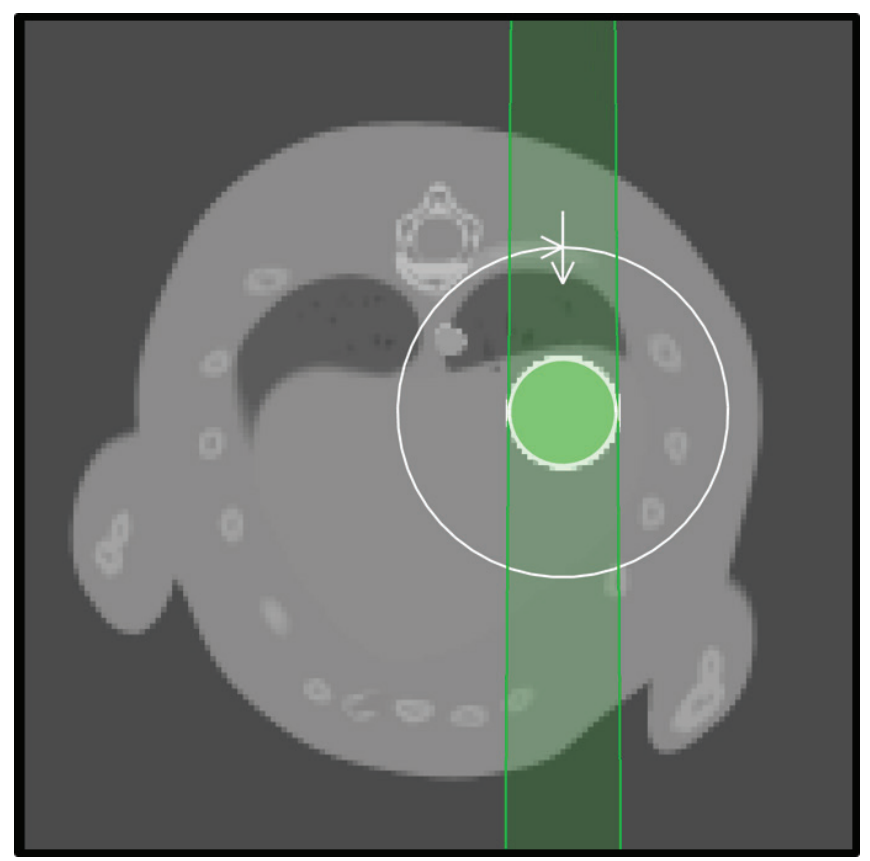

Figure 4.2 - Axial view of the treatment plan. The beam, indicated by the green shaded region, is shaped here by a $4 \mathrm{~mm}$ circular collimator and it rotates $360^{\circ}$ in the plane of the figure around the target, which is located at the isocentre of the irradiator.

Circular brass collimators with diameters ranging from 3-7 $\mathrm{mm}\left(\mathrm{C}_{3}-\mathrm{C}_{7}\right)$ for the $3 \mathrm{~mm}$ tumour and $4-8 \mathrm{~mm}\left(\mathrm{C}_{4}-\mathrm{C} 8\right)$ for the $4 \mathrm{~mm}$ tumour were used. The beam was invariably centred in the tumour position at the first timepoint (maximum exhale, where breathing speed is zero), as shown on Figure 4.2. The target coordinates were kept unchanged for the following timepoints even though the tumour coordinates followed the curves presented on Figures 4.I $(\mathrm{e}, \mathrm{g})$. In other words, the irradiation assumed the tumour was not moving.

The EGSnrc-based Monte Carlo (MC) simulation code DOSXYZnrc ${ }^{[17,18]}$ was used as the dose engine of the TPS. For each time-point, Ioo million photons were generated, and transported down to an energy cutoff (PCUT) of Io $\mathrm{keV}$ and the electron energy cutoff (ECUT) was set to a total energy value of $736 \mathrm{keV}$ (225 kV kinetic energy, meaning no secondary electrons were transported). The calculation time was around 30 minutes per time-point for a full arc in a machine with I2 processors (Intel ${ }^{\circledR}$ Xeon ${ }^{\circledR}$ CPU-X 5660 $2.8 \mathrm{GHz})$ and $16 \mathrm{~GB}$ of RAM. To avoid redundant calculations, only the breathing phase time-points of the anaesthesia curve were simulated; as of 
time-point $\mathrm{I} 2$ on (or as of time $0.6 \mathrm{~s}$ in figure 4.Ie), the geometry remains static. For the tidal breathing, the full curve was simulated due to the absence of a rest phase.

For each time-point, masks of the tumour and OARs were extracted from the TPS. Additionally, the $3 \mathrm{D}$ dose-map, and beam-on time were obtained from the MC calculation. A time-resolved dose was hence derived from the dose distributions in combination with the correlation of organ displacement, the beam and the time-point at a specific time interval, as described at Appendix B of van der Heyden et $\mathrm{al}^{\left[{ }^{[\mathrm{r}]}\right]}$. Therefore, the influence of the beam on space and time, considering gantry rotation and breathing motion was taken into account.

\subsubsection{Metrics and cost function}

The full treatment plan, or the treatment plan to a complete breathing curve, was assessed through cumulative dose-volume histograms (DVHs) of the tumour and OARs time-resolved dose. Additional radiotherapy dose reporting parameters were considered, such as the metrics Dgo, V9o and mean dose to the OARs. The D9o is defined as the dose to the $90 \%$ of the tumour volume; V9o is the percentage of tumour volume which receives a dose equal or higher than $90 \%$ of the prescription dose; and the mean dose to selected OARs is the average dose value in a determined organ volume. Considering the minimum acceptable D9o, V9o and the maximum acceptable mean dose to the OARs, for a lung tumour with a specific size and position, a simple cost function (EqI) was derived. The metrics were employed as constraints (Eq2) to an interactive optimization problem to derive tumour margins, considering breathing motion. The objective was to minimize the cost function tumour-margin by maximizing D9o, V9o and minimizing the mean dose to the OARs. The collimator diameter is the parameter that was optimised through this procedure.

$$
\begin{aligned}
& \text { (1) } \mathrm{M}=\frac{\left(d_{\mathrm{C}}-d_{\mathrm{T}}\right)}{2} \\
& \text { (2) }\left\{\begin{array}{l}
\frac{D 90_{\mathrm{T}}}{\left(D 90_{\mathrm{T}}\right)_{\mathrm{ct}}}\left(d_{\mathrm{C}}\right)>1 \\
\frac{V 90_{\mathrm{T}}}{\left(V 90_{\mathrm{T}}\right)_{\mathrm{ct}}}\left(d_{\mathrm{C}}\right)>1 \\
\frac{\overline{\mathrm{OAR}_{\mathrm{n}}}}{(\overline{\mathrm{DAR}})_{\mathrm{ct}}}\left(d_{\mathrm{C}}\right)<1
\end{array}\right.
\end{aligned}
$$


Where $\mathrm{M}$ is the margin, $d_{\mathrm{c}}$ is the collimator diameter, $d_{\mathrm{T}}$, the tumour diameter, D9от and V9от, $D_{90}$ and $V_{90}$ of the tumour and $O A R_{\mathrm{n}}$, the mean dose to each $O A R_{\mathrm{n}}$, respectively. (D9от) $)_{\mathrm{ct}},\left(\right.$ V9От $_{\mathrm{ct}}$ and $(O A R \mathrm{n})_{\mathrm{ct}}$ are the constraints applied to each of these quantities.

\subsubsection{Four dimensional dose prediction with rigid projection of the first frame}

Additional irradiation parameters, other than the field diameter, could be optimised at the same time, but due to the high computational cost that each simulation requires, this becomes impractical. Thus the need of a fast tool to assess the effect of breathing motion on treatment quality and to generate relevant metrics.

An interpolating function was fitted to the $3 \mathrm{D}$ dose map of the first time point given by $\mathrm{MC}$ calculations (this was done by using the MATLAB function scatteredInterpolant() with the linear method option on the dataset available: the result is a $3 \mathrm{D}$ interpolant that describes the dose of the first time-point as a function of $\mathrm{x}, \mathrm{y}$ and $\mathrm{z}$ ). This function was then projected onto the moving geometry/structures (their movement determined by NURBS) and the accumulated dose was determined.

This approach was intended as a tool to drive the selection of irradiation parameters through an optimisation routine, but it must not lead to absolute results: the accuracy of the estimated doses must be assessed using systematic calculations of the selected configuration.

\subsubsection{CT blurring}

The standard cone-beam CT (СBCT) scanning protocol at our institution uses a gantry speed of $0.5 \mathrm{rpm}$, which yields a CT scan beam-on time of I2O s. In this period, 60 to 300 breathing cycles would occur. To analyse the effect of the long scan period on the tumour shape and the CT blurring, the software VOXelized CT SImulator (VOXSI) ${ }^{[19]}$ was used. Further information on this method is in Appendix A.

\subsection{Results}

\subsection{Full-treatment quality}

Figures $4.3(\mathrm{a}, \mathrm{c})$ and $4.3(\mathrm{~b}, \mathrm{~d})$ present the DVHs for the full treatment plans of the contrasting positions $\mathrm{P}_{\mathrm{I}}$ and $\mathrm{P}_{4}$, respectively. Although the coverage of the tumour (and the OAR) increases with the increasing collimator diameter, a homogeneous dose distribution is never achieved 
for the totality of the volume. This effect is explicit in Figures $4.3(\mathrm{e}, \mathrm{f})$; the tumour dose profiles show the dose gradient in agreement with the motion illustrated in Figure 4.3(g). Additionally, the DVHs of the OARs become unacceptable for larger collimator diameters. As expected, the respiratory motion affects treatment quality; the anaesthesia curve cases at Pi present the worst overall results.

The results for the parameters V9o and D9o, Figures 4.4(a,e,b,f), were in
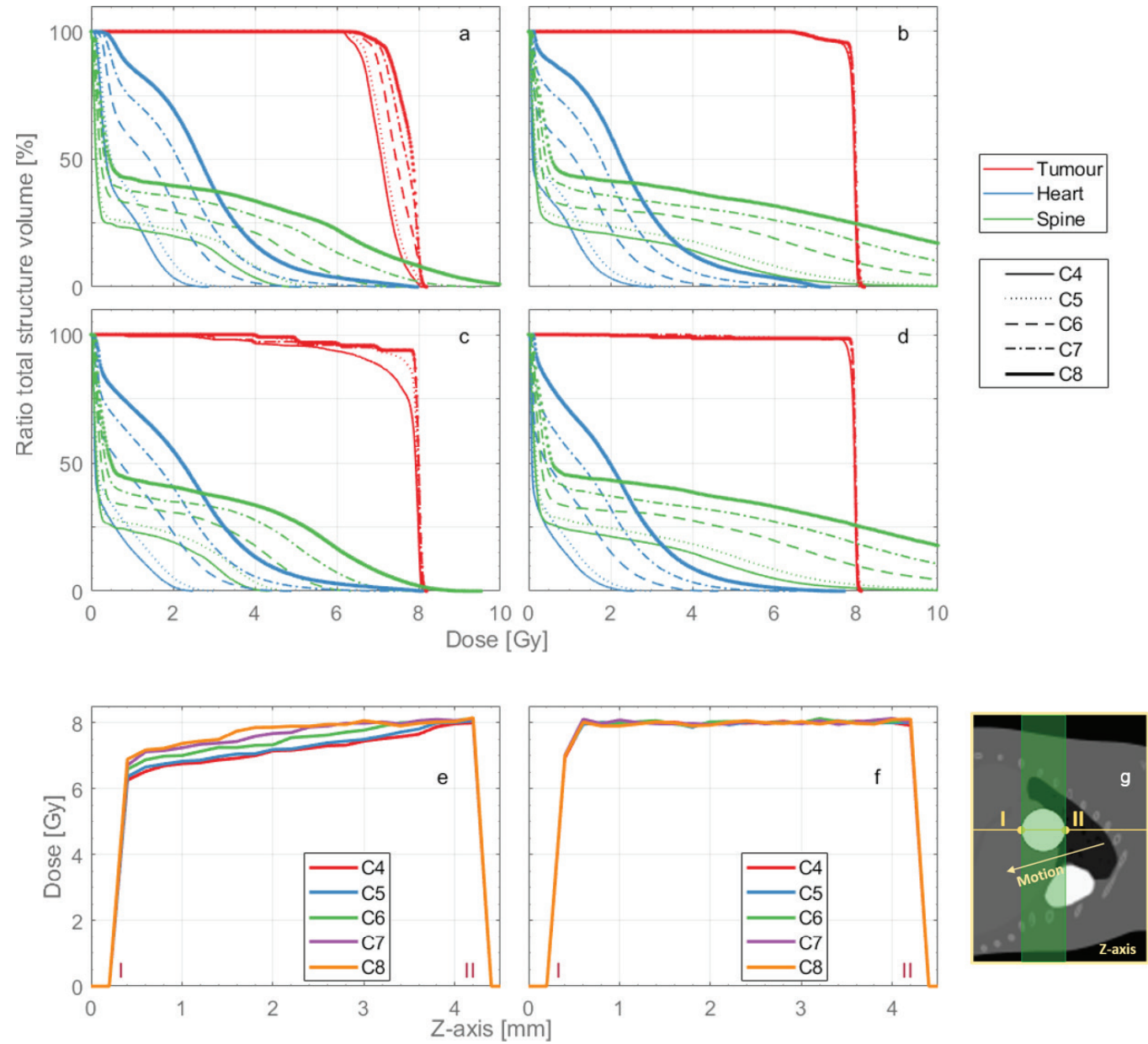

Figure 4.3-Full treatment dose-volume histograms of the $4 \mathrm{~mm}$ tumour for the anaesthesia curve (a) at position I, (b) at position 4; for the tidal curve (c) at position I, (d) at position 4. The different colours represent the different structures and the line styles the different collimator diameter sizes, $4-8 \mathrm{~mm}\left(\mathrm{C}_{4}\right.$-C8). Tumour dose profiles for the $4 \mathrm{~mm}$ tumour at position $\mathrm{I}$ for the (e) anaesthesia and the (f) tidal curve. (g) Representation of the location where the dose profiles were drawn, the roman numerals are displayed in Figures e-g and the arrow indicates the motion direction. 
agreement with the DVH results: they are increasingly lower for sites where motion plays a large role. Additionally, for the cases in which the diameter of the collimator was identical to the diameter of the tumour, both V9o and Dgo presented lower values of coverage and dose.

The mean doses to the OARs (Figures $4.4(\mathrm{c}, \mathrm{d}, \mathrm{g}, \mathrm{h})$ ) increase with the collimator size and vary with the tumour position, as their location can be more or less susceptible to the diameter of the treatment beam.

\subsubsection{Margin function}


Figure 4.4 - DVH metrics for the four different tumour positions, represented by $\mathrm{P}_{\mathrm{I}} \mathrm{P}_{4}$ at the abscissa axis, for the (a-d) $3 \mathrm{~mm}$ tumour and the (e-h) $4 \mathrm{~mm}$ tumour. The circles and the triangles depict the anaesthesia and the tidal curves, respectively. The colours illustrate the different collimator sizes, ranging from $3 \mathrm{~mm}\left(\mathrm{C}_{3}\right)$ to $8 \mathrm{~mm}(\mathrm{C} 8)$. (a,e) show the V9o of the tumour, $(\mathrm{b}, \mathrm{f})$ the Dgo of the tumour, $(\mathrm{c}, \mathrm{g})$ the mean dose to the heart and $(\mathrm{d}, \mathrm{h})$ the mean dose to the spinal cord. The dotted line on Figures $(\mathrm{b}, \mathrm{f})$ indicate the prescription dose of $8 \mathrm{~Gy}$. 
The cost function (Eqsi,2) was applied to the previously presented metrics. In Figure 4.5(a), a generic case with the somewhat arbitrarily selected constraint values for V9o, D9o and mean dose to each OAR of 70\%, 6.5 Gy and 2.5 Gy, is described. The solution domain is represented by the shaded region, where the ratio value of the first pair of tumour constraints is maximised and the value of the remaining OAR constraints is minimized, which yields a minimum collimator size of $5.8 \mathrm{~mm}$ and maximum of 7.6 $\mathrm{mm}$. For a mild case with V9o of $50 \%$, D9o of 5 Gy and mean dose to each OAR of 3 Gy, depicted in Figure 4.5(b), the solution is domain broader, a collimator diameter of 5.I mm would suffice. For extreme cases of tolerance or rigorousness, the solution can be the entire domain or the function may present no solution.

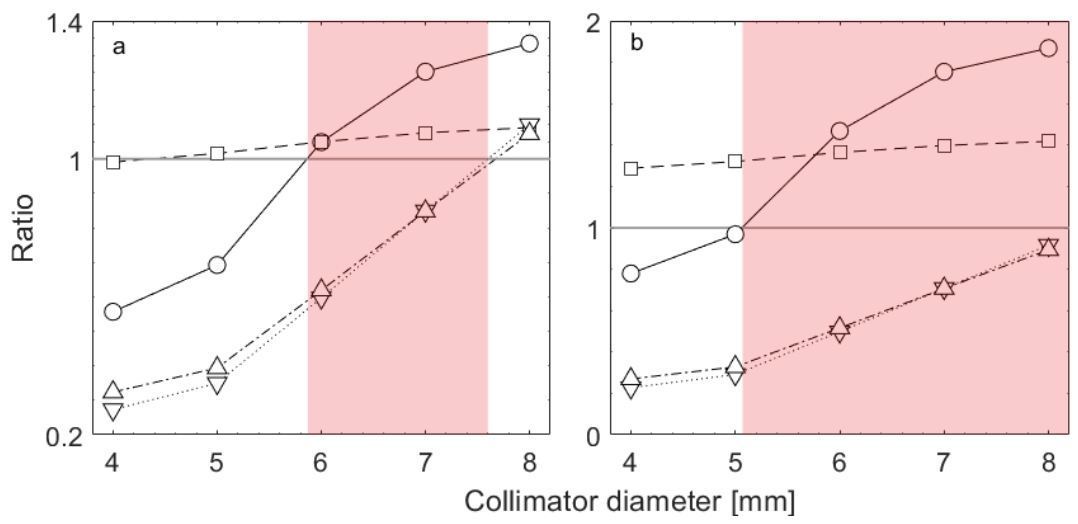

\begin{tabular}{|ll|}
\hline$\bigcirc$ & $V 90_{T} /\left(V 90_{T}\right)_{c t}$ \\
$\square$ & $D 90_{T} /\left(D 90_{T}\right)_{c t}$ \\
$\nabla$ & $\overline{O A R_{(h)}} / \overline{\left(O A R_{(h)}\right)}$ \\
$\triangle$ & $\overline{O A R_{(s)}} / \overline{\left(O A R_{(s)}\right)}$ \\
\hline$c t$
\end{tabular}

Figure 4.5- Graphical representation of the cost function constraint ratios and solution domains (represented by the shaded region), with (a) a generic and (b) a mild case. The circles and the squares illustrate the V9o and D9o constraint ratio to the tumour, respectively. The downward and the upward-pointing triangles illustrate the mean dose constraint ratio to the heart and the spinal cord, respectively. The grey line crossing the ordinate axis at value one represents the boundary, to which the constraints are maximised or minimised. The data for the $4 \mathrm{~mm}$ tumour, PI, anaesthesia curve case was used here. 


\subsubsection{Dose interpolation}

The interpolation function was fitted onto the $\mathrm{MC}_{3} \mathrm{D}$ dose map of the first time point, illustrated by Figure 4.6(a), and projected upon the moving structures, for which the dose distributions are represented by Figures $4 \cdot 6(\mathrm{~b}-\mathrm{d})$.
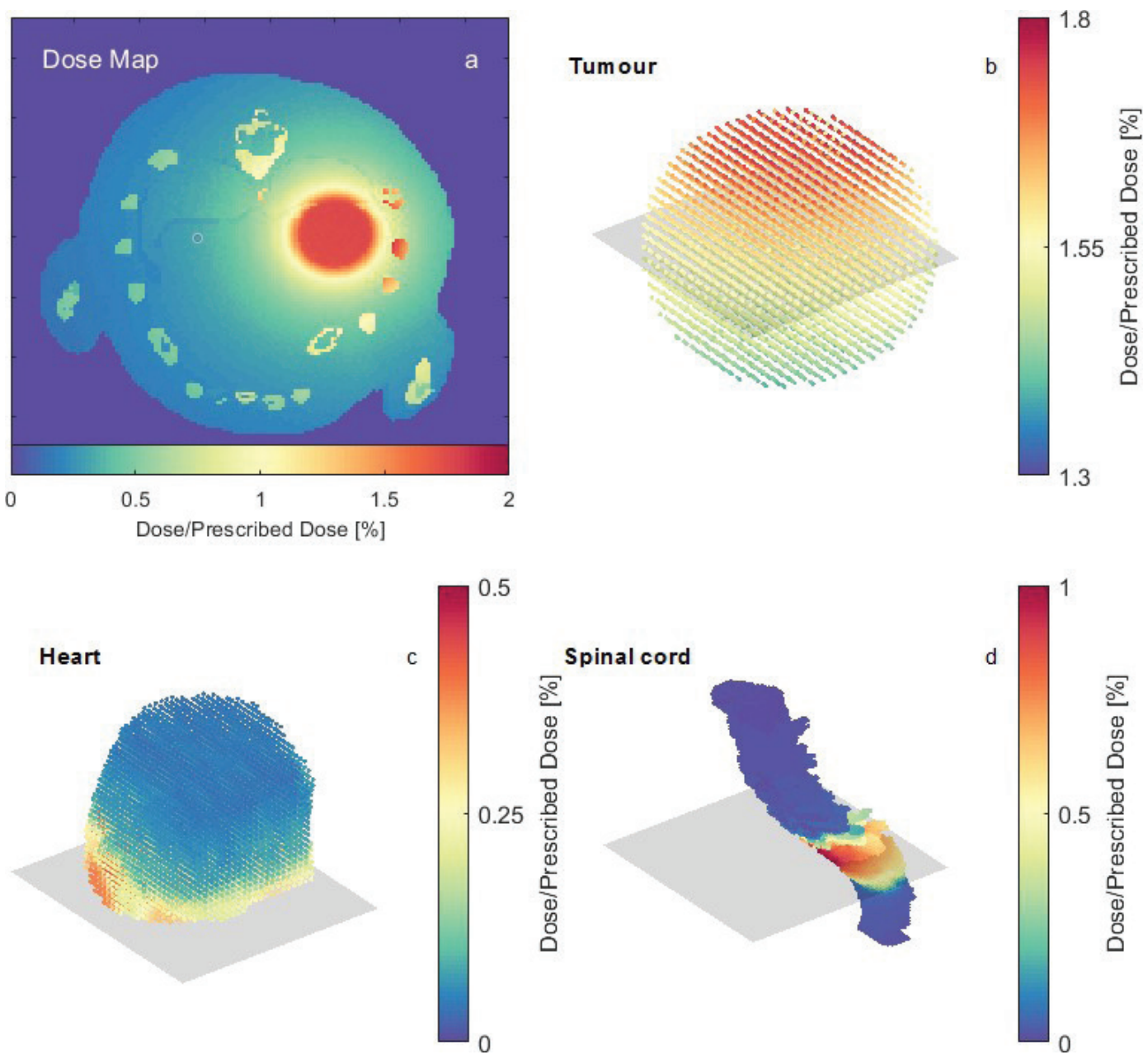

Figure 4.6 - (a) Axial view of the 3 D dose-map of the first time-point. (b-d) Dose distributions at the structures tumour, heart and spinal cord, respectively, at the first time-point. The rectangular shaded region in 6(b-d) represents the slice of the dose-map shown in Figure 4.6(a). Figures 4.6(bd) represent a case with the tumour located at $P_{I}$ and anaesthetized breathing. 
Figures $4.7(\mathrm{a}-\mathrm{c})$ present a comparison between the dose interpolation from a single dose calculation and the full MC results. For the spinal cord, which is less affected by movement, the interpolation showed excellent results, with no relative difference in respect to the MC results. For the tumour and the heart, average DVH differences of $1.5 \%$ and $28.9 \%$ were found, respectively. Figure 4.7 (a) show an overestimation of the heart and tumour DVHs using the interpolation method. The computational time to run the interpolation function on a laptop (Intel ${ }^{\circledR}$ Core $^{\mathrm{TM}}$ i7-5600-CPU $2.6 \mathrm{GHz}, \mathrm{I} 6 \mathrm{~GB}$ of RAM) was ten minutes in contrast to the 4-6 hours for the full MC.

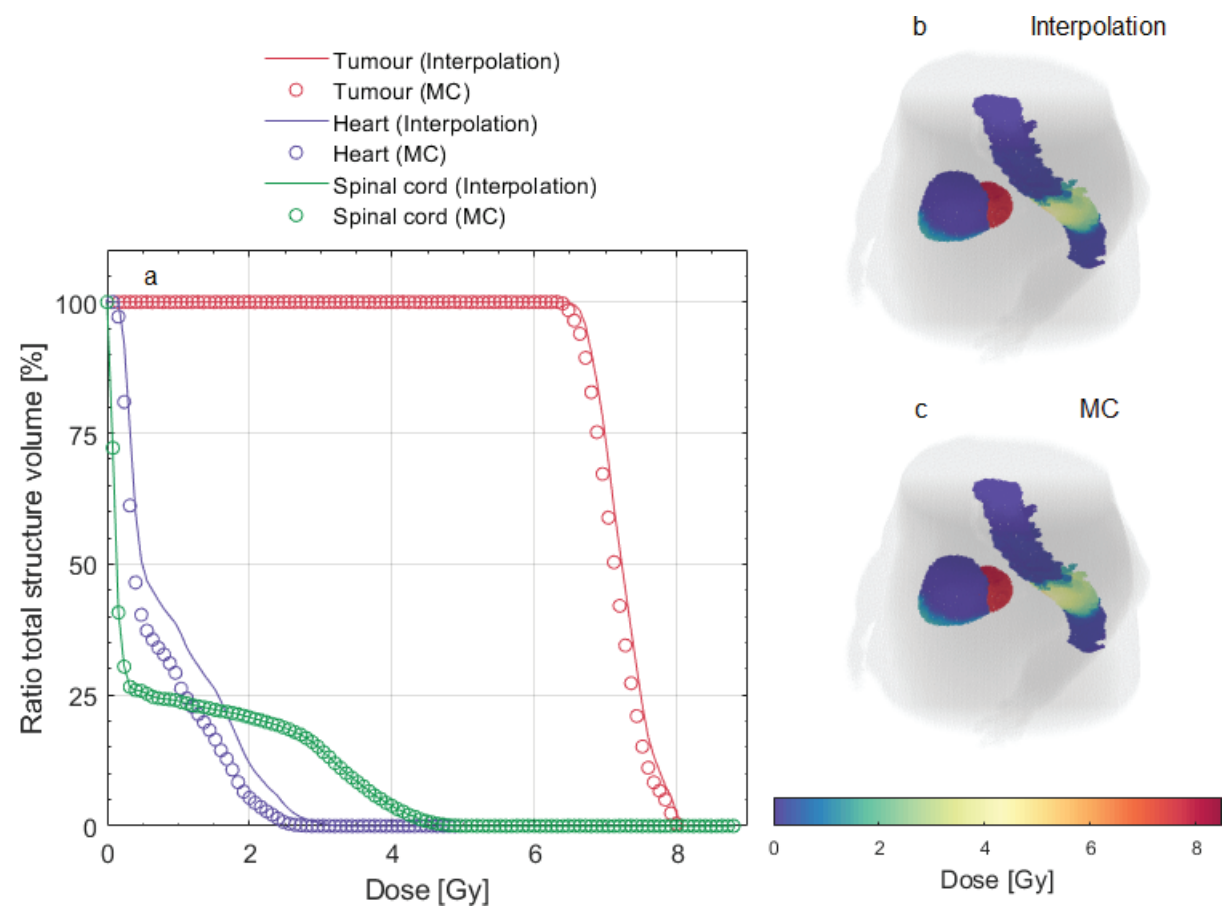

Figure 4.7 - Comparison between the dose interpolation and full MC methods. (a) DVHs of the structures tumour, heart and spinal cord for both methods. (b,c) Interpolation and MC dose at the end of the treatment represented by the dose map of the $3 \mathrm{D}$ structures. 


\subsection{Discussion}

The effect of the respiratory motion on the treatment plan quality is strongly dependent on the breathing pattern and the tumour position. Both breathing curves explored in this study on mathematical phantoms represent real, but somewhat extreme scenarios. It is reasonable to assume that the actual breathing curve of a mouse undergoing CT imaging or irradiation would lie somewhere between these two curves and would vary according to the anaesthesia protocol and the respiratory and cardiac rates the animal would exhibit, as the recommendations for anaesthesia methods vary among institutions ${ }^{[20-23]}$. A sedation scenario with minimal motion while physically restraining the specimen would be ideal.

For pulmonary sites, where substantial influence of anatomical movement is expected, the tumour position itself had an extensive effect on the quality of the treatment plan. Differences of $82 \%$ and $29 \%$ were found in the values of V9o and D9o, respectively, for plans with the same tumour size $(3 \mathrm{~mm})$, breathing curve (anaesthesia) and collimator $\left(\mathrm{C}_{3}\right)$ but different tumour positions ( $\mathrm{P}_{1}$ and $\mathrm{P}_{4}$ ), Figures $4.4(\mathrm{a}, \mathrm{b})$. The plans for $\mathrm{P}_{3}$ and $\mathrm{P}_{4}$ presented the best overall results, while $\mathrm{P}_{1}$ and $\mathrm{P}_{2}$ had invariably a compromise between tumour conformity and health tissue damage. For tumours located at the diaphragm $\left(\mathrm{P}_{\mathrm{I}}\right)$, a PTV margin delineation is strongly recommended to avoid target dose gradients in the tumour itself.

The margin function presented can be a useful tool to ensure treatment conformity and OAR sparing for the various tumour positions. The constraints are adjustable, more sensitive parameters such as D95 and V95 could be employed or other constraints added. Different weights can be applied to the mean dose to the OARs and their values could be based on radiobiological information. This function is independent of the breathing curve; it could be applied for other curves and irradiation regimens. It is also suitable for irradiators with variable collimator sizes. Although only results for circular collimators were presented, it is possible to expand the function to other shapes.

Additional parameters should also be taken into account when establishing the concept of PTV. This study showed a first step towards establishing a margin recipe, which comprises respiratory motion. Setup and fractionation errors should also be estimated and considered. For example, accurate collimator positioning and quality assurance are of paramount importance for proper dose reporting. 
Furthermore, cardio-thoracic sites could benefit from the use of motion tracking and gating techniques for imaging ${ }^{[24]}$ and irradiation ${ }^{[25]}$. Selective acquisition during the rest phase of the breathing cycle, and choosing these intervals for radiation delivery, can be effective in reducing respiratory artefacts and ensure optimal dose delivery to the target and minimal dose to surrounding tissues, especially for orthotopic tumours.

Independently of the margin function, the investigation of an interpolation function for dose accumulation from different anatomic phases to a reference phase using a rigid grid had the purpose of speeding up the dose calculation process. The method, also called direct dose mapping ${ }^{[26]}$, is a first approximation as it does not account for tissue deformation and the cardiac motion was not modelled. The results, an average difference of o, I. 5 and $28.9 \%$ for the structures spinal cord, tumour and heart, between the interpolated and the full MC dose, and calculation time at least ro times faster, are promising for further development of the technique. Results may depend on the targeted site and surrounding anatomy. As this method employs the first frame as the reference phase, in reality, the dose map derived from the CBCT scan would be subject to noise and blurring. This matter was investigated in the supplementary materials, and the effect was found to be minimal, although further assessment would be ideal. The presented technique could potentially be used for adaptive radiotherapy, which requires the daily delivered dose to be accumulated.

\subsection{Conclusion}

Respiratory motion plays an important role in dose conformity when targeting thoracic tumours. It is recommended to add a target margin, especially for tumours on sites where movement is substantial, such as the diaphragm. A simple method was developed to derive tumour margins based on a number of initial user inputs avoiding the need of extensive $\mathrm{MC}$ calculations. It is a first step towards a standard PTV concept in preclinical radiotherapy. The use of direct dose mapping yielded an acceptable approximation for the cases presented, with decreased calculation cost.

In future work, different tumour sites and the influence of other parameters (e.g. collimator shape) could be similarly investigated and a series of visual tools like the one depicted in Fig. 5 could be generated: these would represent a quick aid, available to the end-user for selecting treatment features by simply imposing dose/volume constraints on the tumour or OARs. 


\subsection{Appendix A}

The freely available software VOXSI ${ }^{[27]}$ (research version) simulates CT projections and images of user specified voxelized geometries. This was used here to perform CT simulation of the MOBY phantom. VOXSI allows the user to modify the X-ray spectrum, simulation geometry, CT setup, detector energy response, and image reconstruction parameters. The CT simulation algorithm calculates the X-ray attenuation in each voxel by using the total mass attenuation coefficient as a function of the photon energy and the radiological path length according to Lambert-Beer's attenuation law. Different image reconstruction algorithms are implemented to reconstruct fan-beam projection data. CT imaging pre-sets used in this work are listed in Table 4.Ar, and the Electronic Portal Imaging Device (EPID, XRD o820 $\mathrm{AN}_{3}$ ES, Perkin Elmer ${ }^{\circledR}$, Waltham, MA) response curve were loaded in VOSXI and a realistic level of Poisson noise was added.

\begin{tabular}{|c|c|c|c|}
\hline \multicolumn{4}{|c|}{ VOXSI Simulation Parameters } \\
\hline \multirow{3}{*}{ X-Ray Source } & Peak Energy & 80 & $\mathrm{keV}$ \\
\hline & Angle & $20^{\circ}$ & \\
\hline & Focal Spot & I & $\mathrm{mm}$ \\
\hline \multirow{3}{*}{ Filtration } & Air & 303,6 & $\mathrm{mm}$ \\
\hline & $\mathrm{Be}$ & 0,8 & $\mathrm{~mm}$ \\
\hline & $\mathrm{Al}$ & 2 & $\mathrm{~mm}$ \\
\hline General & Mean Energy & $4 \mathrm{I}, \mathrm{OI}$ & $\mathrm{keV}$ \\
\hline \multicolumn{4}{|c|}{ Moby phantom } \\
\hline \multirow{2}{*}{ Geometry } & Slice thickness & 0,2 & $\mathrm{~mm}$ \\
\hline & Number of slices & IOI & \\
\hline \multirow{5}{*}{ Setup } & Source to origin & 303,6 & $\mathrm{~mm}$ \\
\hline & Detector to origin & 622,9 & $\mathrm{~mm}$ \\
\hline & Number of detectors & IO24 & \\
\hline & FOV diameter & IOO & $\mathrm{mm}$ \\
\hline & Number of projections & 602 & \\
\hline \multirow{3}{*}{ Simulation } & Current & 2,5 & $\mathrm{~mA}$ \\
\hline & period & $\mathrm{I} 2 \mathrm{O}$ & \\
\hline & Cupping correction & yes & \\
\hline \multirow{3}{*}{ Reconstruction } & Type & Filtered Back-projection & \\
\hline & Filter & Hann (o.5) & \\
\hline & Interpolation method & Spline & \\
\hline
\end{tabular}

Table 4.AI -CT scan parameters added to the software VOXSI 
Figure 4.Ar.I shows simulated CT images for an extreme case of motion: a tumour located along the diaphragm ( $\mathrm{P}_{\mathrm{I}}$ ) for an anaesthetized MOBY phantom. This extreme case serves to establish an upper limit to the severity of image blurring. The full anaesthesia curve, including a long rest phase (Figure 4.2(e)) was simulated, Figure 4.Ar.I(a), and although the edges of the organs are not as sharp as in a MOBY phantom and the role of noise is noticeable, the blurring does not modify the tumour shape or increase its diameter. However, when only the breathing phase is simulated, Figure 4.Ar.r(b), the full motion amplitude is apparent. The blurring is excessive and contouring any of the structures in this image would be challenging. For the case of a single time point in the rest phase, Figure 4.AI.I(c), where the phantom is still, the simulated scan is consonant with the full curve, Figure 4.Ar.I(a), in terms of structure definition and size.

Overall, the effect of respiratory motion on the CT scan blurring can be considered minimal using this method; and the shape of the tumour is highly preserved. For the tidal breathing curve, Figure 4.2(g), as motion plays a minor role the same outcome is expected. A thorough investigation on this effect would be ideal, as VOXSI is designed for fan-beam geometries. Instead of averaging the different frames, a projection based ${ }_{4} \mathrm{D}-\mathrm{CBCT}$ simulation would be ideal.
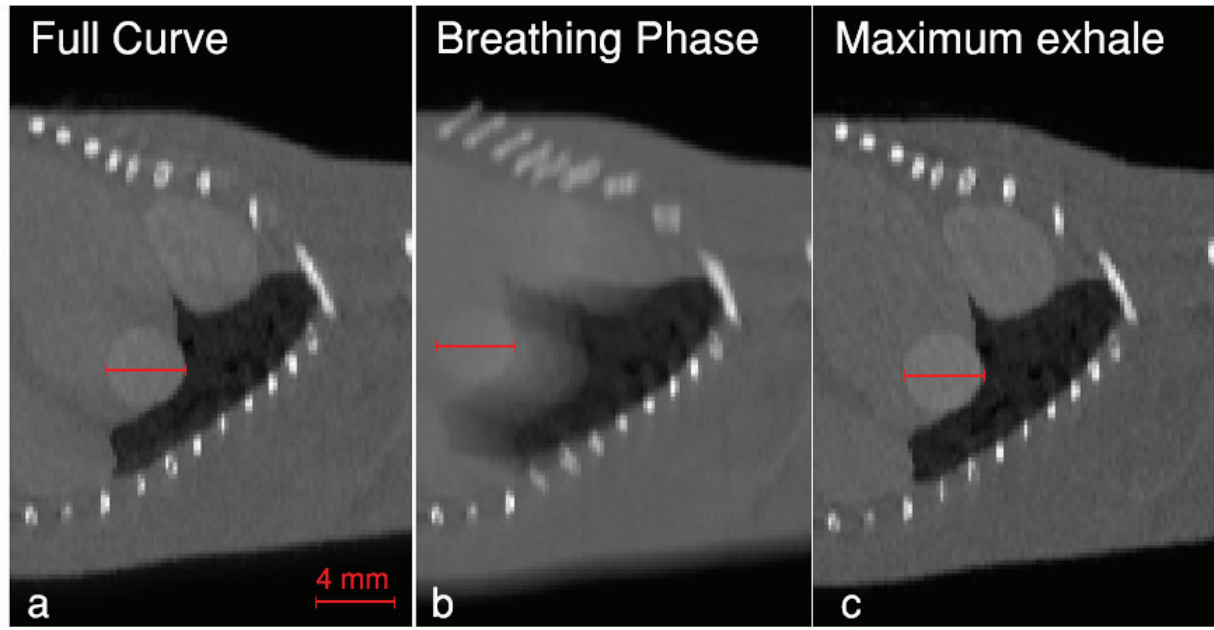

Figure 4.Ar.I - Simulated CT images of a MOBY phantom (with a $4 \mathrm{~mm}$ tumour) using VOXSI. (a) The full anaesthesia curve. (b) Only the breathing phase. (c) The point of maximum exhale, at the end of the breathing and throughout the rest phase. 


\section{$4 \cdot 7$ References}

${ }^{[\mathrm{r}]}$ F. Verhaegen, L. Dubois, S. Gianolini, M.A. Hill, C.P. Karger, K. Lauber, K.M. Prise, D. Sarrut, D. Thorwarth, C. Vanhove, B. Vojnovic, R. Weersink, J.J. Wilkens, D. Georg, ESTRO ACROP: Technology for precision small animal radiotherapy research: Optimal use and challenges, Radiother Oncol, I26 (2018) 47I-478.

${ }^{[2]}$ T. Landberg, P. Almond, J.M.V. Burgers, M. Busch, C.A. Joslin, J.P. Paunier, Report 29, Journal of the International Commission on Radiation Units and Measurements, osi5 (1978) NP-NP.

${ }_{33}$ T. Landberg, J. Chavaudra, J. Dobbs, J.P. Gerard, G. Hanks, J.C. Horiot, K.A. Johansson, T. Möller, J. Purdy, N. Suntharalingam, H. Svensson, Report 62, Journal of the International Commission on Radiation Units and Measurements, os32 (1999) NP-NP.

${ }^{[4]}$ T. Landberg, J. Chavaudra, J. Dobbs, G. Hanks, K.A. Johansson, T. Möller, J. Purdy, Report 50, Journal of the International Commission on Radiation Units and Measurements, os26 (1993) NP-NP.

${ }^{[5]}$ M. van Herk, P. Remeijer, C. Rasch, J.V. Lebesque, The probability of correct target dosage: dose-population histograms for deriving treatment margins in radiotherapy, Int J Radiat Oncol Biol Phys, 47 (2000) II2I-II35.

[6] A.L. McKenzie, M. van Herk, B. Mijnheer, The width of margins in radiotherapy treatment plans, Phys Med Biol, 45 (2000) 333I-3342.

[7] J.C. Stroom, H.C. de Boer, H. Huizenga, A.G. Visser, Inclusion of geometrical uncertainties in radiotherapy treatment planning by means of coverage probability, Int J Radiat Oncol Biol Phys, 43 (I999) 905-919.

${ }^{[8]}$ J. Stroom, K. Gilhuijs, S. Vieira, W. Chen, J. Salguero, E. Moser, J.J. Sonke, Combined recipe for clinical target volume and planning target volume margins, Int J Radiat Oncol Biol Phys, 88 (2014) 708-7I4.

[9] J.J. Gordon, J.V. Siebers, Convolution method and CTV-to-PTV margins for finite fractions and small systematic errors, Phys Med Biol, 52 (2007) I967-1990.

${ }^{\left[{ }^{\circ \circ]}\right.}$ M. van Herk, Errors and margins in radiotherapy, Semin Radiat Oncol, I4 (2004) 52-64. ${ }^{[\text {[r] } W . P . ~ S e g a r s, ~ B . M . ~ T s u i, ~ E . C . ~ F r e y, ~ G . A . ~ J o h n s o n, ~ S . S . ~ B e r r, ~ D e v e l o p m e n t ~ o f ~ a ~ 4-D ~ d i g i t a l ~}$ mouse phantom for molecular imaging research, Mol Imaging Biol, 6 (2004) I49-I59.

${ }^{\left[{ }_{2}\right]}$ D.R. White, J. Booz, R.V. Griffith, J.J. Spokas, I.J. Wilson, Report 44, Journal of the International Commission on Radiation Units and Measurements, Volume os23 (I989) NP.

${ }^{\left[{ }_{3}\right]}$ A. Vaniqui, L. Schyns, I.P. Almeida, B. van der Heyden, S.J. van Hoof, F. Verhaegen, The impact of dual energy CT imaging on dose calculations for pre-clinical studies, Radiat Oncol, I2 (2017) I8I.

$\left.{ }^{\left[{ }^{1}\right]}\right]$ B. van der Heyden, S.J. van Hoof, L.E.J.R. Schyns, F. Verhaegen, The influence of respiratory motion on dose delivery in a mouse lung tumour irradiation using the ${ }_{4} \mathrm{D}$ MOBY phantom, Br J Radiol, 9o (2017) 20160419.

${ }^{\left[{ }_{5}\right]}$ S.J. van Hoof, P.V. Granton, F. Verhaegen, Development and validation of a treatment planning system for small animal radiotherapy: SmART-Plan, Radiother Oncol, Io9 (2013) 36I-366.

[r6] C.M. Ma, C.W. Coffey, L.A. DeWerd, C. Liu, R. Nath, S.M. Seltzer, J.P. Seuntjens, M. American Association of Physicists in, AAPM protocol for $40-300 \mathrm{kV}$ x-ray beam dosimetry in radiotherapy and radiobiology, Med Phys, 28 (2001) 868-893. 
${ }^{\left[{ }_{17}\right]}$ C. Ma, P. Reckwerdt, M. Holmes, D. Rogers, B. Geiser, DOSXYZ users manual, DOI (1995).

${ }^{[18]}$ I. Kawrakow, D. Rogers, The EGSnrc code system: Monte Carlo simulation of electron and photon transport, DOI (2000).

${ }^{[19]}$ B. van der Heyden, L.E.J.R. Schyns, M. Podesta, A. Vaniqui, I.P. Almeida, G. Landry, F. Verhaegen, VOXSI: a voxelized single- and dual-energy CT scenario generator for quantitative imaging, Phys and Imag Radiat Oncol, DOI (Accepted (2018)).

${ }^{[20]}$ J.L. Tremoleda, A. Kerton, W. Gsell, Anaesthesia and physiological monitoring during in vivo imaging of laboratory rodents: considerations on experimental outcomes and animal welfare, EJNMMI Res, 2 (2012) 44.

${ }^{[21]}$ A. Tsukamoto, K. Serizawa, R. Sato, J. Yamazaki, T. Inomata, Vital signs monitoring during injectable and inhalant anesthesia in mice, Exp Anim, 64 (2015) 57-64.

${ }^{[22]}$ University of Minnesota, Anesthesia Guidelines: Mice, DOI (2018).

${ }^{\left.{ }_{23}\right]}$ Michigan Medicine Confluence, Guidelines on Anesthesia and Analgesia in Rats, DOI (2013).

[24] S.H. Bartling, J. Kuntz, W. Semmler, Gating in small-animal cardio-thoracic CT, Methods, 50 (2010) 42-49.

${ }^{\left.{ }^{225}\right]}$ M.A. Hill, J.M. Thompson, A. Kavanagh, I.D.C. Tullis, R.G. Newman, J. Prentice, J. Beech, S. Gilchrist, S. Smart, E. Fokas, B. Vojnovic, The Development of Technology for Effective Respiratory-Gated Irradiation Using an Image-Guided Small Animal Irradiator, Radiat Res, I88 (2017) 247-263.

${ }^{[26]}$ H.S. Li, H. Zhong, J. Kim, C. Glide-Hurst, M. Gulam, T.S. Nurushev, I.J. Chetty, Direct dose mapping versus energy/mass transfer mapping for ${ }_{4} \mathrm{D}$ dose accumulation: fundamental differences and dosimetric consequences, Phys Med Biol, 59 (2014) I73-I88. ${ }^{\left[{ }_{27}\right]}$ B. van der Heyden, L.E.J.R. Schyns, M. Podesta, A. Vaniqui, I.P. Almeida, G. Landry, F. Verhaegen, VOXSI: a voxelized CT scenario generator for imaging and dose calculations in radiotherapy, Phys and Imag Radiat Oncol, DOI (Submitted (2018)). 

Dose to water versus dose to medium from cavity theory applied to small animal irradiation with kilovolt $x$-rays

Ana Vaniqui, Blake R Walters, Gabriel P Fonseca, Frank Verhaegen

Physics in Medicine and Biology

August 2019, 64165001 


\section{Abstract}

Several dose calculation algorithms exist in the small animal precision irradiation field where kilovolt $(\mathrm{kV})$ photon energies are used. Dose reporting is a current matter of concern as the different dose descriptors dose-to-water-in-medium $\left(D_{\mathrm{wm}}\right)$ and dose-to-medium-in-medium $\left(D_{\mathrm{mm}}\right)$ coexist, due to historical and practical reasons. For $\mathrm{kV}$ photons differences between both quantities are expected to be amplified due to the difference in photon energy absorption coefficients for different media, and could represent a limiting factor for accurate translation of pre-clinical research into clinical trials, since currently knowledge is lacking on which descriptor correlates best with radiation damage. The main goal of this study was to analyse the relationship between $D_{\mathrm{mm}}$ and $D_{\text {wm }}$ for $\mathrm{kV}$ irradiation of small animals, using different flavours of the intermediate cavity theory (ICT). ICT corresponds to a mix of small (SCT) and large (LCT) cavity theory contributions.

Mathematical phantoms were designed with different voxel sizes, considering the pre-clinical practice and extreme scenarios, and a selection of materials, broad enough to include the range of densities and elemental compositions conventionally encountered in animal studies. An ex-vivo mouse chest irradiation was included in the study, due to relevant tissue compositions and densities, and the presence of heterogeneous regions. A modified version of the Monte Carlo code DOSXYZnre was used to derive $D_{\mathrm{mm}}$ and convert to $D_{\mathrm{wm}}$ using ICT. Furthermore, local photon spectra were generated in different regions of the chest case.

Depending on the energy and the size of the cavity, which in this work we equate to the voxel size, $D_{\mathrm{mm}}$ ranged from 0.68 to 4.37 times $D_{\mathrm{wm}}$. The combination of higher $\mathrm{kV}$ energy with a very small cavity size yielded a decreased $D_{\mathrm{mm}}$ in comparison to $D_{\mathrm{wm}}$, this behaviour was reversed for larger cavities combined with lower $\mathrm{kV}$ energies. Hence, the impact of 
the cavity dimensions on estimated $D_{\mathrm{wm}}$ is significant on pre-clinical $\mathrm{kV}$ beams. $D_{\mathrm{mm}}$ and $D_{\mathrm{wm}}$ in the ex-vivo male mouse were found to differ by $-29 \%$ to $286 \%$. For both phantom and specimen the variation between dose descriptors is significant for tissues which diverge from water in terms of density and atomic composition, e.g. bones and adipose. Caution is advised when using the IC theory as there is a lack of consensus on methods to derive the weighting factor (parameter, $\mathrm{d}$ ) of the SCT and LCT; for the same irradiation conditions, the use of different $d$-values yielded $D_{\text {wm }}$ differences of up to $20 \%$.

Pre-clinically, such divergence between dose descriptors could enable biological damage. Moreover, the abiding debate over which quantity to favour is foreseen to linger while it is not clear which quantity correlates better with the biological effects of ionizing irradiation: pre-clinical radiotherapy might represent an ideal platform for measurement-based studies to settle this fundamental question. Finally, dose distribution comparisons should use the same reporting quantity and caution regarding reported quantity and dose comparisons is advised. 


\subsection{Introduction}

Traditionally, the human body has been assumed water-equivalent for the purpose of simplifying dose calculations in radiotherapy ${ }^{[\mathrm{l}]}$. Dose calculation methods have moved away from measurement-based approaches towards sophisticated model-based dose calculation (MBDC) algorithms such as collapsed cone convolution or Monte Carlo (MC) simulations. These algorithms transport radiation through heterogeneous media and report the absorbed dose to a reference medium embedded in a surrounding transport medium ${ }^{[2]}$.

Pursuant to the evolution of dose calculation methods, different dose descriptors have been derived using cavity theory and coexist to date. They are referred to as dose-to-medium-in-medium $\left(D_{\mathrm{mm}}\right)$, dose-to-water-inmedium $\left(D_{\mathrm{wm}}\right)$ or dose-to-water-in-water $\left(D_{\mathrm{ww}}\right)$, which acknowledge the dose scoring and the surrounding media as arbitrary or water. Figure 5.I (d) depicts the different descriptors. For $D_{\mathrm{mm}}$ the scoring volume is filled with the transport medium. $D_{\mathrm{wm}}$ considers the reference medium as water during energy deposition scoring, yet not during photon transport. $D_{\mathrm{ww}}$ transports and scores in water.

Over the last decades, the increased commercial availability of MBDC algorithms has started a debate over whether dose-to-medium $\left(D_{\mathrm{mm}}\right)$ should replace dose-to-water $\left(D_{\mathrm{wm}}\right.$ or $\left.D_{\mathrm{ww}}\right)$ as the absorbed dose in treatment plans, since most MBDC algorithms report $D_{\mathrm{mm}}$ as their natural scoring quantities ${ }^{[3-8]}$. Arguments supporting medium-based protocols include: the conversion factor from medium to water (when $D_{\mathrm{wm}}$ is desired, starting from calculated $D_{\mathrm{mm}}$ ) adds additional uncertainty; $D_{\mathrm{mm}}$ might provide a better correlation to biological response ${ }^{[5]}$; and the change would not affect most clinical protocols, as nearly all tissues are similar to water for external beam radiotherapy (EBRT) using megavolt (MV) photon beams. Advocates in opposition claim that clinical experience on tumour and healthy tissue response as well as dosimetry protocols are based on $D_{\mathrm{wm}}$ (or even $D_{\mathrm{ww}}$ ), hence its use allows traceability back to historical data; radiosensitive structures are embedded in cells which are approximately water; and, when converting from CT numbers to media, there is an intrinsic uncertainty to tissue type and composition, potentially making the medium unknown ${ }^{[8]}$. In EBRT reported differences between water- and medium-based dose calculations are only significant for osseous tissues ${ }^{[6,9]}$, therefore the American Association of Physicists in Medicine Task Group IO $^{[\mathrm{II}]}$ report does not take a stand in favour of reporting either $D_{\mathrm{wm}}$ or $D_{\mathrm{mm}}$ : it recommends that the 
reported quantity is specified along with information to enable conversion between quantities. The situation is more critical at photon energies in the kilovoltage range $(\mathrm{kV})$, especially below $200 \mathrm{kV}$, which comprises brachytherapy, orthovoltage treatments, radiology imaging including lowenergy mammography, and small animal radiotherapy ${ }^{[\mathrm{Io,II}]}$. The latter is used for pre-clinical evaluation of advanced radiotherapy techniques ${ }^{[\mathrm{In}, \mathrm{rl}]}$ from both a technical and relative biological effectiveness (RBE) perspective $e^{\left[r_{3}\right]}$. In general, current commercial solutions permit irradiations using energies up to 220 or $225 \mathrm{kVp}$ and specimens are imaged using sub-millimetre resolution CT scanners due to their small dimensions.

In the $\mathrm{kV}$ range, the mass-energy absorption coefficients $\left(\mu_{\mathrm{en}} / \rho\right)$ of several human tissues differ reasonably from that of water and vary so rapidly with energy and between media that the ratio of medium and water coefficients $\left(\mu_{\mathrm{en}} / \rho\right)_{\mathrm{w}}$ has to be evaluated over the entire local photon spectrum; an average value would be misleading ${ }^{\left[r_{4}\right]}$. Thus, assigning all tissues to water may introduce significant dose errors. Additionally, the RBE for this energy range is increased, as low-energy photons are more potent per unit dose in causing biological damage to living organisms than higher energy photons $s^{[5]}$. Consequently, absorbed dose conversion among dose descriptors should be implemented with great care.

Conversion factors between the different dose descriptors can be derived using a full MC calculation or, theoretically, considering the pertinent cavity theory. Target size, atomic composition and mass density define the choice of small, large or intermediate cavity theory, which in turn depends on the target size relative to secondary charged particle ranges. Tedgren and Carlsson ${ }^{[16]}$ interpreted the cavity as being of nuclear $(\sim \mathrm{IO} \mu \mathrm{m})$ or cellular $(\sim$ IOO $\mu \mathrm{m})$ dimensions while Oliver and Thomson ${ }^{[17]}$ assumed cavity dimensions in the $\leq \mathrm{IO} \mu \mathrm{m}$ range, considering the potential radiobiological impact of cell nucleus DNA-bound water ( nm-sized volumes) and water targets in subcellular compartments ${ }^{[1,6,17]}$. For brachytherapy, Landry ${ }^{[2]}$ has used large cavity theory (LCT) for the $D_{\mathrm{mm}}$ to $D_{\mathrm{wm}}$ conversion, while Tedgren and Carlsson ${ }^{[\mathrm{r} 6]}$ recommended small cavity theory (SCT) and Fonseca ${ }^{\left[{ }^{14}\right]}$ showed that application of SCT and LCT is dependent on tissue type and local photon energy fluence variation. Enger ${ }^{[\mathrm{rol}]}$ compared SCT and LCT predictions to nuclear-sized cavities.

In the context of small animal irradiation and to the best knowledge of the authors, research on different dose descriptors and application of cavity 
theory has not been published. We present a first study following the recent dose reporting recommendations ${ }^{[19]}$ context as different dose calculation methods exist in this field, e.g. analytical, superposition-convolution and $\mathrm{MC}^{[\mathrm{r2}]}$. This study uses a modified version of the MC code DOSXYZnrc ${ }^{[20]}$ to derive the dose descriptors $D_{\mathrm{mm}}$ and $D_{\mathrm{wm}}$ using different approaches to the ICT. Simulations parameters are set to represent the behaviour of a micro irradiator X-RAD $225 \mathrm{Cx}$ (Precision X-Ray, North Branford (CT), United States). The voxels comprising the phantom and the cavity are assumed to have equal dimensions. Calculations are performed for low-kV energies, where divergence among dose descriptors is augmented, and could be a limiting factor for the accurate translation of pre-clinical research into clinical trials. A theory section is included to aid in the MC code rationale.

\subsection{Theory}

The absorbed dose is the expected energy imparted to an infinitesimal volume of matter by ionizing radiation, taken at each point of an irradiated medium $^{[2]}$. For a photon fluence spectrum, electronic kerma $\left(K_{\mathrm{el}}\right)$, the net energy transferred to charged particles, is a good approximation of absorbed dose in the medium $\left(D_{\mathrm{m}}\right)$ if there is charged particle equilibrium (CPE). CPE exists when the fluence of charged particles liberated inside and leaving the volume is balanced, in number and energy, by particles liberated elsewhere entering the volume. For a photon fluence spectrum under CPE, the absorbed dose in the medium is given by equation (I)

$$
K_{\mathrm{el}} \stackrel{\mathrm{CPE}}{=} D_{\mathrm{m}} \stackrel{\mathrm{CPE}}{=} \int_{0}^{E_{\mathrm{max}}} E\left[\Phi_{E}(E)\right]_{\mathrm{m}}\left[\frac{\mu_{\mathrm{en}}(E)}{\rho}\right]_{\mathrm{m}} \mathrm{d} E
$$

where $\left[\mu_{\mathrm{en}}(E) / \rho\right]_{\mathrm{m}}$ is the medium mass-energy absorption coefficient, $\left[\Phi_{E}(E)\right]_{\text {med }}$ is the fluence, differential in energy $(E)$ and $E_{\max }$ is the maximum energy of the photon spectrum. For charged particle beams, under $\delta$-ray equilibrium ( $\delta$-eqm, less demanding and existing if there is $\mathrm{CPE}$ ), the corresponding absorbed dose is given by equation (2):

$$
D_{\mathrm{m}} \stackrel{\delta-\text { eqm }}{=} \int_{0}^{E_{\mathrm{max}}}\left[\Phi_{E}(E)\right]_{\mathrm{m}}\left[\frac{S_{\mathrm{el}}(E)}{\rho}\right]_{\mathrm{m}} \mathrm{d} E
$$

where $\left[\mathrm{S}_{\mathrm{el}}(E) / \rho\right]_{\mathrm{m}}$ is the medium unrestricted mass electronic stopping power. 
Considering an arbitrary volume element, a cavity consisting of a medium different from the surrounding one, in a medium subject to an unperturbed photon radiation field, the size of the cavity, relative to the range of charged particles crossing it, is instrumental in the determination of the factor which correlates the dose to the cavity embedded in the medium $\left(D_{\text {cav.m }}\right)$ and the dose to the medium $\left(D_{\mathrm{m}, \mathrm{m}}\right)$ at the location of the cavity in the absence of it, equation (3). Figure 5.I schematically summarizes the cavity theories: small, large and intermediate.

$$
f_{\mathrm{cav}, \mathrm{m}}=\left(\frac{D_{\mathrm{cav}, \mathrm{m}}}{D_{\mathrm{m}, \mathrm{m}}}\right)
$$

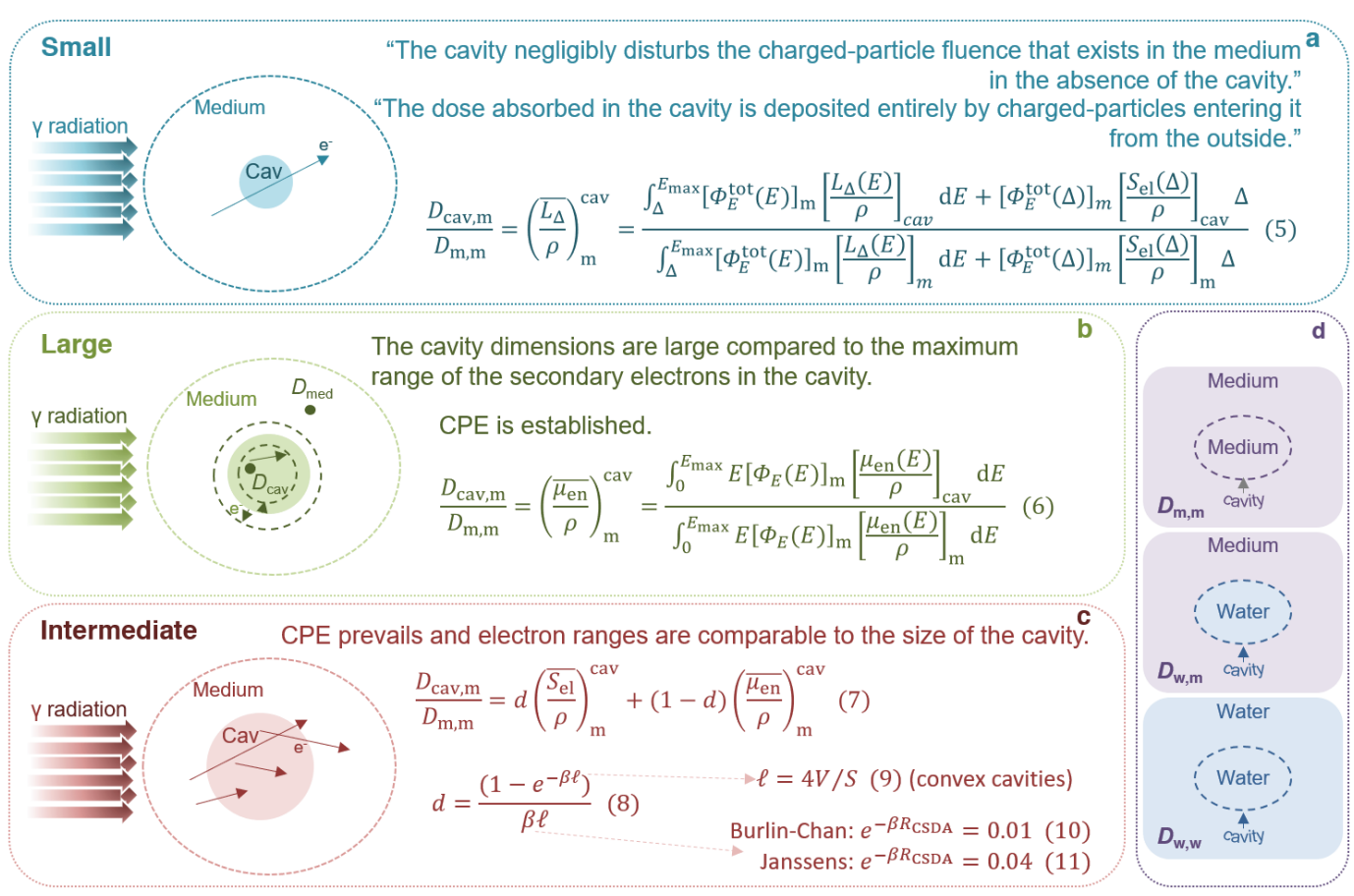

Figure 5.I - (a) small, (b) large and (c) intermediate cavity theory graphical representation, main conditions and equations. Adapted from Andreo et al. ${ }^{[22]}$. (d) Representation of the dose descriptors dose-to-medium-in-medium, $D_{\mathrm{m}, \mathrm{m}}$, dose-to-water-in-medium, $D_{\mathrm{w}, \mathrm{m}}$, and dose-to-water-in-water, $D_{\mathrm{w}, \mathrm{w}}$.

1 The term 'electronic' (e.g. kerma and stopping power) is used instead of the term 'collisional', following recommendations from ${ }^{[22]}$ P. Andreo, D.T. Burns, A.E. Nahum, J.P. Seuntjens, F.H. Attix, Fundamentals of Ionizing Radiation Dosimetry, Wiley-VCH, Berlin, 2017. 


\subsection{Small cavities (SC)}

Small cavities, Figure 5.I(a), fulfilling so called Bragg-Gray (BG) conditions, do not disturb the fluence of secondary electrons in the medium: throughout the cavity the electron fluence is approximately the same as that in the medium in the absence of the cavity. The BG conversion factor is given by, $f_{\text {cav, }, \text {, }}^{\mathrm{BG}}$ equation $(4)^{[23]}$

$$
f_{\mathrm{cav}, \mathrm{m}}^{\mathrm{BG}}=\frac{D_{\mathrm{cav}, \mathrm{m}}}{D_{\mathrm{m}, \mathrm{m}}}=\left(\frac{\overline{S_{\mathrm{el}}}}{\rho}\right)_{\mathrm{m}}^{\mathrm{cav}}=\frac{\int_{0}^{E_{\max }}\left[\Phi_{E}(E)\right]_{\mathrm{m}}\left[\frac{S_{e l}(E)}{\rho}\right]_{c a v} d E}{\int_{0}^{E_{\max }}\left[\Phi_{E}(E)\right]_{m}\left[\frac{S_{e l}(E)}{\rho}\right]_{m} d E}
$$

where $S_{\mathrm{el}}(E) / \rho$ is the unrestricted mass electronic (collisional) stopping power, $\left[\Phi_{\mathrm{E}}(E)\right]_{\mathrm{m}}$ is the primary electron fluence in the medium, assumed to be the same as in the cavity and $\left(S_{\mathrm{el}}(E) / \rho\right)_{\mathrm{m}}^{\text {cav }}$ is the ratio of the unrestricted stopping power for cavity and medium.

The BG theory, which assumes that either all electrons are deposited locally or there is $\delta$-eqm, was modified by Spencer and Attix (SA) to explicitly consider energetic $\delta$-rays. A further formulation ${ }^{[23]}$ included track-end terms, as there was no distinction in BG between secondary and knock-on electrons entering the cavity. The SA conversion factor is given by $f_{\text {cav, } \mathrm{m}}^{\mathrm{LC}}$, Figure 5.I (a), equation $(5)^{[22]}$, where $L_{\Delta}(E) / \rho$ is the restricted mass electronic stopping power for energy losses lower than $\Delta,\left[\Phi_{\mathrm{E}}^{\mathrm{tot}}\right]_{\mathrm{m}}$ is the total electron fluence in the medium considering all electrons with $\mathrm{E} \geq \Delta$ and $\left(\overline{L_{\Delta}} / \rho\right)_{\mathrm{m}}^{\mathrm{cav}}$ is the ratio of the restricted mass electronic stopping power for cavity and medium. $\Delta$ is often chosen as a cut-off energy of an electron that has a range just sufficient to cross the cavity.

With somewhat arbitrarily chosen values, $\Delta$ depends on the size of the cavity and is chosen so that electrons with $\mathrm{E}>\Delta$ tend to pass through the cavity and deposit their energy in the medium whereas the ones with $\mathrm{E}<\Delta$ are locally absorbed. The SA theory is the most sophisticated SCT theory available $e^{[16]}$. In comparison to the unrestricted (BG), the restricted (SA) mass electronic stopping-power ratio improved agreement with experimental data ${ }^{[24]}$ and it handles large atomic composition differences between medium and cavity. 


\subsubsection{Large cavities (LC)}

A cavity with dimensions greater than the maximum range of the secondary electrons is considered a large cavity (LC) ${ }^{[2]}$, shown in Figure 5.I(b). In this case, the cavity does not perturb the primary photon fluence and CPE is established. The conversion factor is given by $f_{\text {cav } m}^{\mathrm{LC}}$ Figure 5.I (b), equation (6), where $(\overline{\mu \mathrm{er}} \rho)_{\mathrm{m}}^{\mathrm{cav}}$ is the spectrum-averaged mass-energy absorption coefficient ratio for cavity and medium, $\mu_{\mathrm{en}}(E) / \rho$ is the mass energy absorption coefficient, $\left[\Phi_{\mathrm{E}}(E)\right]_{\mathrm{m}}$ the photon fluence in the medium assumed to be the same as in the cavity.

\subsubsection{Intermediate cavities (IC)}

In the previous two sections absorbed dose was delivered by secondary electrons either mostly completely traversing the (small) cavity or which start and stop within the (large) cavity. In practice, however, often the absorbed dose is delivered by a combination of the aforementioned phenomena. The Burlin ${ }^{[2]}$ or intermediate cavity (IC) theory handles these cases, Figure 5.I(c).

The IC theory is valid if both medium and cavity are homogeneous, although their compositions may differ. CPE must be established and equilibrium spectra of secondary electrons in medium and cavity must be the same ${ }^{[22]}$. The conversion factor is given by $f_{\text {cavm }}^{\mathrm{LC}}$, equation 7, Figure 5.I (c), where d defines a weighting factor that can vary from zero to unity and determines the extent to which the cavity fulfils BG conditions and the extent to which CPE dominates, so d weighs the contributions from SC and LC theories. The parameter $d$ is determined according to Figure 5.I (c), equation (8), where $\beta$ is the mass absorption coefficient of secondary electrons and $\ell$ the mean chord length, which for convex cavities is taken as Figure 5.I(c), equation (9), where $\mathrm{V}$ and $\mathrm{S}$ are the cavity volume and surface area, respectively. The validity of the IC theory also relies on the assumption that cavity and medium are subjected to an omnipresent, homogeneous photon field: it generates a secondary electron fluence, attenuated exponentially (with $-\beta \ell$, at a distance $\ell$ ) as it penetrates the cavity, while the electron spectrum generated in the cavity builds up exponentially to $\left(1-e^{-\beta \ell}\right)$ of its equilibrium value ${ }^{[25]}$.

The parameter $\beta$ can be calculated using equations of the form $e^{-\beta R}=x$, where $\mathrm{x}$ is a fixed attenuation constant that represents the fraction of particles which reach a depth $\mathrm{R}$, the extrapolated range corresponding to the maximum energy of the electron spectrum. The Burlin-Chan ${ }^{[26]}$ and 
the Janssens ${ }^{[27]}$ methods use $\mathrm{x}=\mathrm{O}$. $\mathrm{OI}$ and $\mathrm{x}=0.04$, respectively: they consider residual electron transmissions of $\mathrm{i} \%$ and $4 \%$. Although maximum energy of the electron spectrum is the prevailing parameter for range determination, Burlin-Chan considered that the relevant spectrum of electrons in cavity theory is not monoenergetic and has abundant electron energies lower than the maximum energy ${ }^{[26]}$. The Janssens method is considered to be more rigorous as it considers electron backscatter at the interface between different media. Although other methods exist ${ }^{[16,28,29]}$, this work focuses on the Burlin-Chan, equation (го), and the Janssens, equation (II), methods with $R$ taken as $R$ CSDA the continuous slowing down approximation (CSDA) range of electrons of energy equal to that of incident photons (or maximum electron energy), together with a definition of range as $1 \%$ or $4 \%$ particle transmission.

\subsection{Methods}

A modified version of the MC code DOSXYZnrc was used to derive the dose descriptors $D_{\mathrm{mm}}$ and $D_{\mathrm{wm}}$ using different approaches to the ICT, described in the previous section. To analyse the differences between the dose descriptors, a number of phantoms of different voxel sizes were generated in addition to an ex-vivo mouse CT scan. Both phantoms and mouse were segmented using a range of tissues typical of a highly heterogeneous chest region.

\subsection{DOSXYZnrc modifications}

The EGSnrc-based ${ }^{[30]}$ Monte Carlo (MC) simulation code DOSXYZnrec ${ }^{[20]}$, was initially modified to allow the scoring in a user-specified reference medium (a cavity) in medium (e.g. Figure 5.I(d), middle, for the reference medium water, $D_{\mathrm{wm}}$ ), in addition to $D_{\mathrm{mm}}$, using SA formulation and respecting BG conditions. Further modifications are described in the next sections and allow the scoring of the reference medium using Burlin's IC theory. Hereon the term reference medium is used as a synonym of cavity.

\section{5·3.I.I Stopping power ratios}

To score dose deposition in two different media when the cavity is filled with the transport medium, the energy deposition may have distinct modes: it may consider events where energy is deposited by electrons in a step with energy $(E)$ greater than $\Delta$ or events in which the electron starts a step with $E>\Delta$ and ends with $E<\Delta$. ${ }^{\left[{ }^{1}\right]}$ 
The modified DOSXYZnrc calculates the fluence-averaged stopping power ratio, for each charged particle step depositing energy in the medium, $E D E P_{\mathrm{m}}$ (excluding fluorescent photons and not tracking charged particles created with kinetic energy $<\Delta$ ), using:

$$
\left(\bar{L}_{\Delta}\right)_{\mathrm{m}}^{\mathrm{ref}}=f \frac{L_{\Delta_{r e f}}\left(E_{\mathrm{mid}}\right)}{L_{\Delta_{m}}\left(E_{\text {mid }}\right)}+(1-f) \frac{L_{\Delta_{r e f}}(\Delta)}{L_{\Delta_{m}}(\Delta)}
$$

where $f$ is the fraction of the step over which the particle kinetic energy is higher than $\Delta, E_{\text {mid }}$ is the kinetic energy at the mid-point of this fraction of the step. Note that here stopping powers are not normalized by medium density. As CSDA is assumed, $E_{\text {mid }}=\left(E_{\min }+E_{\max }\right) / 2$ or $E_{\text {mid }}=\left(\Delta+E_{\max }\right) / 2$ for steps where the particle kinetic energy is higher or lower than $\Delta$, respectively. $\left(\bar{L}_{\Delta} / \rho\right)_{\mathrm{m}}^{\mathrm{ref}}$ is calculated at the end of the simulation by dividing the cumulative value of $E D E P_{\text {ref }}$ by the cumulative value of $E D E P_{\mathrm{m}}$ and normalizing by the $\rho_{\mathrm{ref}} / \rho_{\mathrm{m}}$. This on-the-fly technique for scoring avoids the need of energy binning of secondary electron fluence to evaluate (5) directly - a similar method is used by the EGSnrc code SPRRZnrc ${ }^{\left[{ }^{3}\right]}$.

\subsection{I.2 Mass-energy absorption coefficients}

For the CPE contribution, equation (6) is evaluated for the calculation of $\left(\overline{\mu_{\mathrm{en}}} / \rho\right)_{\mathrm{m}}^{\mathrm{ref}}$. For each photon step in a cavity, the energy fluence, $\left[\psi_{\mathrm{E}}(E)\right]_{\mathrm{m}}$, where $\psi_{\mathrm{E}}(E)=\Phi_{\mathrm{E}}(E) \cdot E$, is given by the product of the total pathlength of the photon step, the photon energy $(E)$, and the particle's statistical weight. This result is multiplied by $\mu_{\mathrm{en}}(E) / \rho$, interpolated from user-supplied $\mu_{\mathrm{en}}(E) / \rho$ vs $E$ data for reference and local media. At the end of the simulation, the division between the cumulative values (summed over all particles and energies $)$ of $\left[\psi_{\mathrm{E}}(E)\right]_{\mathrm{m}}\left[\mu_{\mathrm{en}}(E) / \rho\right]_{\mathrm{ref}}$ and $\left[\psi_{\mathrm{E}}(E)\right]_{\mathrm{m}}\left[\mu_{\mathrm{en}}(E) / \rho\right]_{\mathrm{m}}$ yields the $\left[\overline{\mu_{\mathrm{en}}} \rho\right]_{\mathrm{m}}^{\mathrm{ref}}$. In comparison to (6), the evaluation of the integrals of $(7)$ is much simpler since photons do not directly deposit energy. Photon fluence is not spread out over multiple energy bins.

\subsubsection{Extra outputs}

In addition to the dose map containing $\mathrm{D}_{\mathrm{mm}}$, the modified DOSXYZnrc outputs $3 \mathrm{D}$ matrices of $D_{(\mathrm{ref}, \mathrm{m})},\left(\overline{S_{\mathrm{el}}} / \rho\right)_{\mathrm{m}}^{\mathrm{ref}}$ and $\left(\overline{\mu_{\mathrm{en}}} / \rho\right)_{\mathrm{m}}^{\mathrm{ref}}$. The code also outputs the maximum kinetic energy of electrons, $R_{C S D A}$ of these electrons in the reference medium, and all the cumulative data required to calculate $D_{(\text {ref, } m)}$, $\left(\overline{S_{\mathrm{el}}} / \rho\right)_{\mathrm{m}}^{\mathrm{ref}},\left(\overline{\mu_{\mathrm{en}}} / \rho\right)_{\mathrm{m}}^{\mathrm{ref}}$ and to estimate $\mathrm{d}$. Two estimates of the parameter $\mathrm{d}$ are output, calculated using a combination of equations (9) and either (IO) or (II). 


\subsubsection{Simulation parameters}

The calculation parameters were set to represent the behaviour of an X-RAD ${ }_{225}$ Cx (Precision X-Ray, North Branford (CT), United States) (r2,32] $^{[21}$ micro irradiator and CT scanner. It is a dual-focus X-ray tube with a 200 angled stationary target made of tungsten, $0.8 \mathrm{~mm}$ beryllium exit window and tube potential of $225 \mathrm{kVp}$ (225 Cx, Comet, Switzerland). An additional $0.32 \mathrm{~mm}$ copper filter is used for irradiation ${ }^{[33]}$; the distance between source and isocentre is fixed at $303.6 \mathrm{~mm}$.

\subsubsection{MC inputs}

The dose calculations considered a parallel photon beam with rectangular collimation $(5 \mathrm{xI} \mathrm{cm})$ that impinged on the geometries from the bottom. The number of histories yielded calculation uncertainties below $5 \%$ for the smallest voxels and $<\mathrm{I} \%$ for larger ones ( $\mathrm{IO}^{9}-\mathrm{IO}^{\mathrm{Io}}$ histories, sampled at the exit plane of the collimator) with no particle recycling and no dose scored in air. Photons were transported down to an energy cutoff (PCUT) of I keV and electrons to a total energy cutoff (ECUT) of $5^{\mathrm{I} 2} \mathrm{keV}$ ( $\mathrm{k} \mathrm{keV}$ kinetic energy, meaning secondary electrons with $\mathrm{E}>\mathrm{I} \mathrm{keV}$ were transported). Figure 5.2(a) shows the irradiation photon spectra of 80,120 , and $225 \mathrm{kVp}$, calculated using SpekCalc ${ }^{[34,35]}$. 


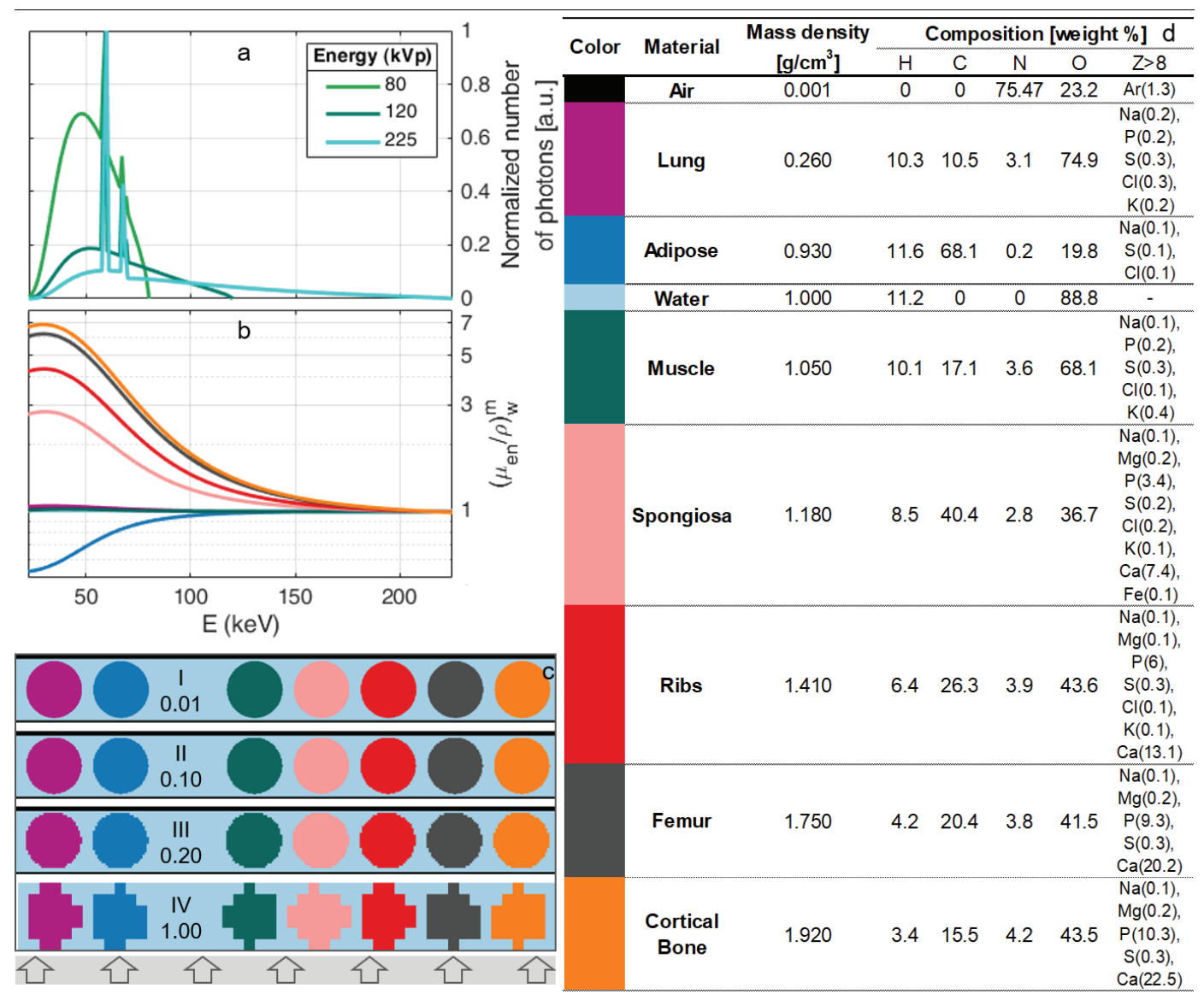

Figure 5.2 - (a) Photon energy spectra used in dose calculations. (b) $\mu$ en $(E) / \rho$ vs $E$ values generated with the g-code. (c) Phantom representation: they were all designed with $48.5 \mathrm{~mm}$ (length) x 6.0 $\mathrm{mm}$ (height) x $5.0 \mathrm{~mm}$ (width), with 0.0I, 0.IO, 0.20 and I.00 $\mathrm{mm}$ isotropic voxel matrices, I, II, III and IV, respectively. The grey box and the arrow below IV indicate the direction and the size of the beam (of 5xi cm, designed to encompass the full geometry). (d) Reference values of mass density $(\rho)$ and elemental composition of phantom and mouse tissue-substitute materials. Note that the colours, which present the materials in (d) are replicated in (b) and (c) to depict the same materials. 
The mean chord length $(\ell)$ calculated according to equation (9) depended upon phantom voxel size. Delta $(\triangle)$ was set to $A E=5 \mathrm{I} 2 \mathrm{keV}$, where $A E$ is the minimum energy for which cross section data has been generated. For this study, $\mathrm{d}$ values were calculated analytically with $R_{\text {CSDA, }}$ taken at the maximum kinetic energy of electrons for each spectrum. A log-log interpolation, with natural cubic splines, was applied on ICRU Report 90 (2016) R CSDA values. For each energy spectrum and cavity size $(\ell)$, R CSDA values were input in equations (Io) or (II) and (8) to generate $d$ values. They were used at run time to convert $D_{(\mathrm{m}, \mathrm{m})}$ to $D_{(\mathrm{ref}, \mathrm{m})}$. Finally, files containing $\mu_{\mathrm{en}}(E) / \rho$ vs $E$ for each medium were used in the simulations. These files were calculated using the EGSnrc user code, g, which generates $\left(E \cdot \mu_{\text {en }}(E)\right) / \rho$ vs $E$ for a given medium. g-code calculations were run with $\mathrm{AE}=5 \mathrm{I}^{2} \mathrm{keV}, \mathrm{AP}=\mathrm{I}$ $\mathrm{keV}$ (the lowest energy for photon transport) and uncertainty of о.оoг\%. Figure $5.2(\mathrm{~b})$ depicts the $\mu_{\mathrm{en}}(E) / \rho$ vs $E$ data used in this study.

\subsubsection{EGS Phantoms}

The cavity is considered to have the same dimensions as one isotropic phantom voxel, considering that voxel dimensions define the maximum resolution of a dose calculation. Mathematical phantoms were conceived to investigate the different cavity theories and the current pre-clinical practice: isotropic voxel sizes of o.or, o.IO, o.20 and I.oo mm were used. All phantoms had length $=48.5 \mathrm{~mm}$, height $=6.0 \mathrm{~mm}$ and width $=5.0 \mathrm{~mm}$ and were designed to model eight cylindrical inserts with $5.0 \mathrm{~mm}$ diameter (similar to preclinical phantoms $\left.\mathrm{s}^{[37}\right)$ and $5.0 \mathrm{~mm}$ length. Figures $5.2(\mathrm{c}, \mathrm{d})$ show the phantom geometries and the media, for which atomic composition and density were defined according to ICRU Report $44^{[38]}$, and the cross-sections to XCOM database. Human tissue compositions and density are commonly used preclinically as small animal tissue information lacks in the literature ${ }^{[5,39]}$. Additional simulations were made using the CT scan of an ex-vivo male mouse, segmented using the materials of Figure 5.2(d) (and a Hounsfield Unit to mass density calibration curve ${ }^{[37]}$ ), with voxel sizes of o.IO $\mathrm{mm}$, the highest resolution of the X-RAD $225 \mathrm{Cx}$ cone beam CT (CBCT) imager, and $0.20 \mathrm{~mm}$, a common value employed by multiple research centres ${ }^{[40]}$. A region comprising the spinal cord and lungs was selected to demonstrate differences in all investigated tissues. 

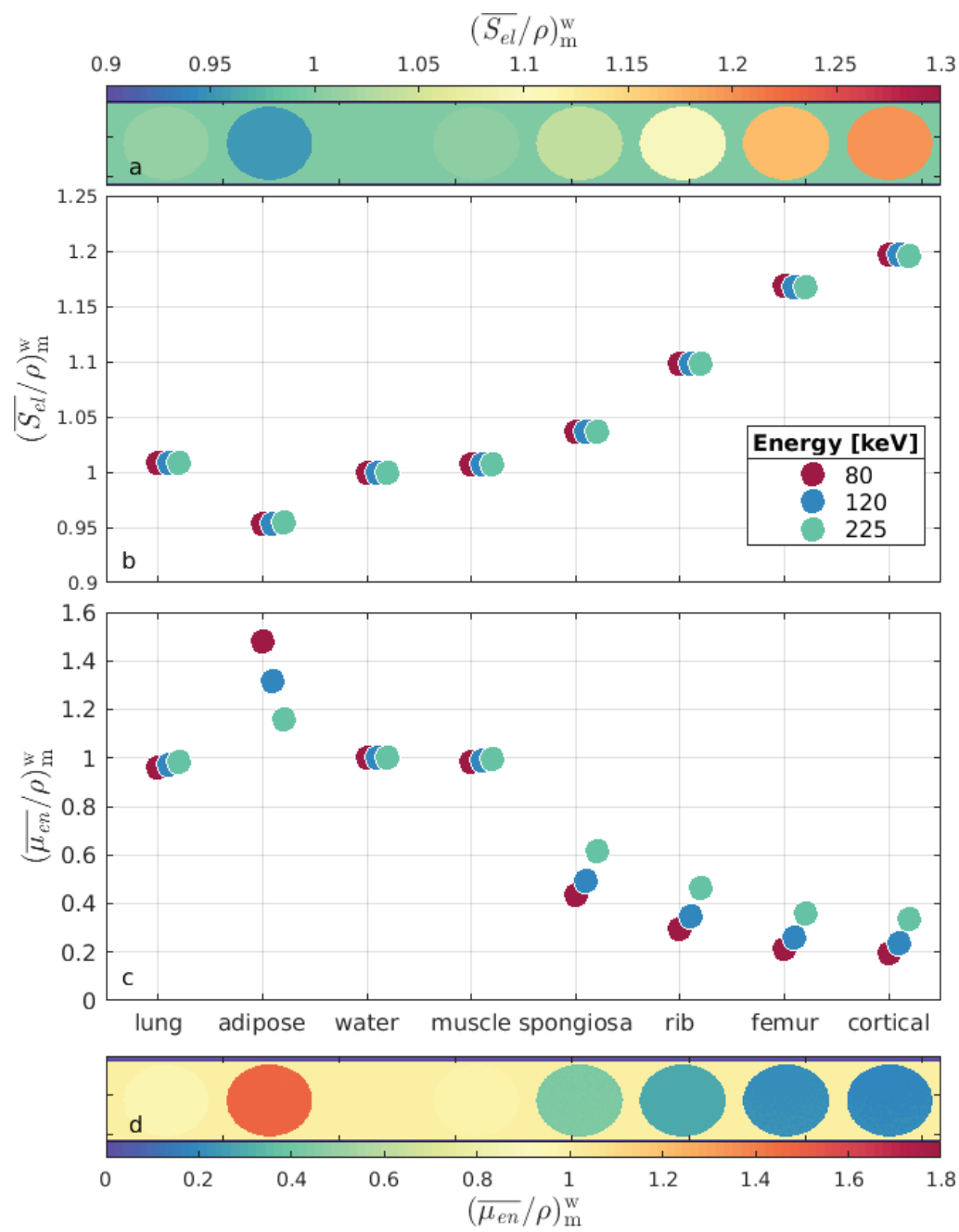

Figure $5.3-(\mathrm{a}, \mathrm{d}) \quad\left(\frac{\overline{S_{\mathrm{el}}}}{\rho}\right)_{\mathrm{m}}^{\mathrm{w}}$ and $\left(\frac{\overline{\mu_{\mathrm{en}}}}{\rho}\right)_{\mathrm{m}}^{\mathrm{w}}$ maps given as output of the dose calculation and $(\mathrm{b}, \mathrm{c})$ mean value per insert for the four different resolution phantoms used in this study. The phantom was irradiated with a beam impinging on it from the bottom, as indicated in Figure 5.2(c). 


\subsubsection{Additional inputs}

A secondary effect, the difference between photon spectra at various locations within the mouse CT scan was investigated and is presented in Appendix Ar.

\subsection{Results}

\subsection{Dose calculation outputs}

Figures $5 \cdot 3(\mathrm{a}, \mathrm{d})$ show the fluence-averaged water-to-medium stopping power ratios, $(\bar{S} / \rho)_{\mathrm{m}}^{\mathrm{w}}$, and ratios of mass-energy absorption coefficients, $\left(\overline{\mu_{\mathrm{en}}} / \rho\right)_{\mathrm{m}}^{\mathrm{w}}$ given as dose calculation outputs. Figures $5 \cdot 3(\mathrm{c}, \mathrm{d})$ show the mean value of $(\bar{S} / \rho)_{\mathrm{m}}^{\mathrm{w}}$ and $\left(\overline{\mu_{\mathrm{en}}} / \rho\right)_{\mathrm{m}}^{\mathrm{w}}$ for each tissue-substitute material. The results are invariant with voxel or cavity size and, for $(\bar{S} / \rho)_{\mathrm{m}}^{\mathrm{w}}$ with energy in the selected range.

Values of the weighting parameter $\mathrm{d}$ for varying cavity mean chord lengths $(\ell)$ are displayed as a function of photon energy in Figure 5.4(a) and as a function of the cavity size (or $\ell$ ) in $4 \mathrm{~b}$. As $d$ determines the extent to which a cavity behaves as a SC (or, alternatively, $1-d$ assesses the degree of CPE in a cavity), figure 5.4(a) shows that $d$ values consistently increase with an increase in energy: higher $d$ values determine the predominance of the SC (first) term of equation (7) over the second. Figure 5.4(b) emphasizes this behaviour: decreasing $\mathrm{d}$ values with cavity size expansion, the LC (second) term of equation (7) becomes gradually dominant. Phantoms I and IV show the two extreme scenarios: $d$ values of 0.98 and 0.04 , respectively. Phantoms II and III, representative of pre-clinical practice, fit well within the IC theory with a considerable $d$ variation depending on energy and $d$ derivation method. For example, for 80 and $225 \mathrm{kV}, d$ was found to be $0.4 \mathrm{I}$ and 0.83 , respectively, for phantom II using Janssens' method. According to the method of calculation, Janssen or Burlin-Chan theories, the numeric difference of $d$ is not negligible, for phantom for III, a variation of $42 \%$ and $15 \%$ for 80 and $225 \mathrm{kV}$, respectively, was found.

Both Figures 5.4(c) and 5.4(d) are outputs from the MC calculations. Figure 5.4(c) shows the range of electrons in water as a function of their maximum kinetic energy: the MC code used these values to calculate the kinetic energy of electrons with a range equal to $\ell$ and the values of $d$ based on $b$ using equations (IO) and (II). Figure 5.4(d) presents the kinetic energy of charged particles with a range correspondent to the mean chord length. Figure 5.4(e) presents the ratio between $D_{\text {wm }}$ dose maps calculated using Janssens or Burlin-Chan methods for the different phantoms and energies. 
Large differences in $d$ values for intermediate sized voxels (phantoms II and III) translated into large relative dose differences. For example, for 80 $\mathrm{kV}$, the average differences for cortical bone and adipose are $20 \%$ and $-4 \%$ for phantom II, conversely for $225 \mathrm{kV}$, the differences are $5.4 \%$ and $-\mathrm{I} .3 \%$, respectively.

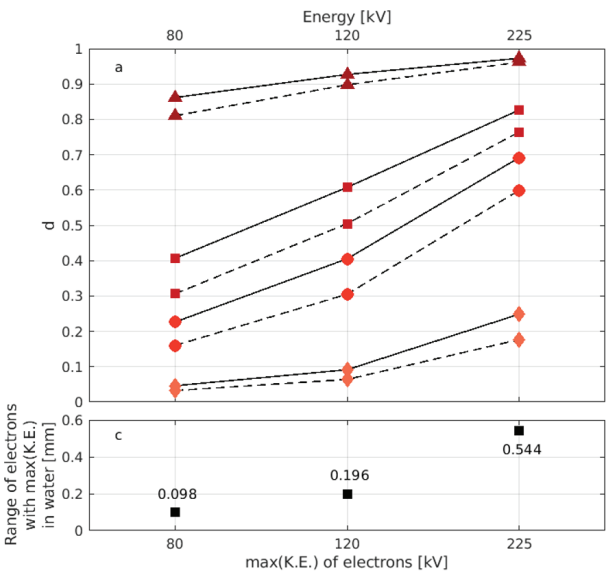

e

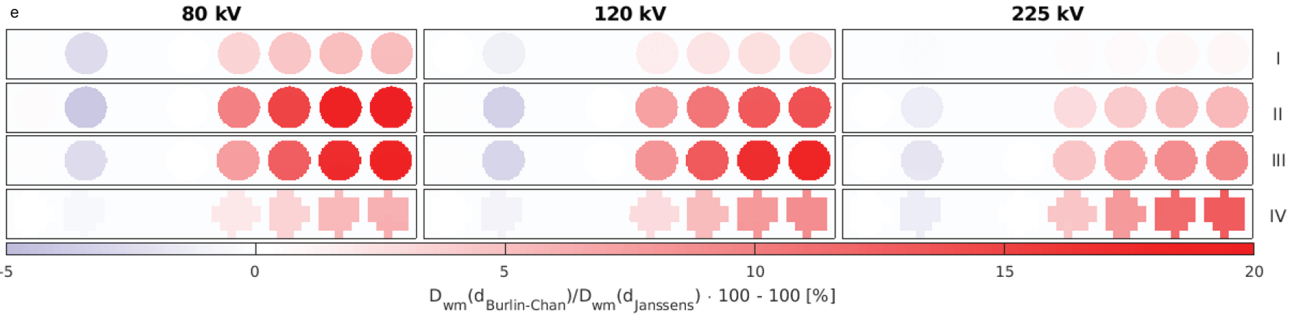

Figure 5.4 - Parameter $d$ a) as a function of energy and identified (with symbols in red shades) by the cavity size, b) as a function of cavity size and identified (with triangles in blue shades) by the energy spectrum. The solid and dashed lines represent Janssens and Burlin-Chan methods, respectively. c) Range of electrons in water as a function of the maximum kinetic energy. d) Kinetic energy of charged particles with a range correspondent to the mean chord length $\boldsymbol{\ell}$. e) Differences between $D_{\text {wm }}$ maps calculated with $d$ parameter values derived from Janssens and Burlin-Chan methods. From top to bottom, each row represents one voxel size phantom (I-IV) and from left to right, each column represents one energy (80, I20 and $225 \mathrm{kVp})$. 


\subsection{2 $\boldsymbol{D}_{\mathbf{m m}} / \boldsymbol{D}_{\mathbf{w m}}:$ Phantom}

Figures $5.5(\mathrm{a}-\mathrm{d})$ depict the $D_{\mathrm{mm}} D_{\mathrm{wm}}$ average estimate for each medium in phantoms I-IV, respectively, the three selected energies and with the two $d$ deriving methods. To graphically highlight the differences between the descriptors, the percentual difference between $D_{\mathrm{mm}}$ and $D_{\mathrm{wm}}$ (instead of between $D_{\mathrm{wm}}$ and $\left.D_{\mathrm{mm}}\right)$ is depicted. For soft tissues, except adipose, the relationship between dose descriptors is equal or close to unity for all different scenarios; an expected behaviour as a consequence of the similarities in terms of atomic composition and density between the media and water (Figure 5.2(d)), small cross section variations, and the predominance of incoherent photon interactions. Results for adipose and bony tissues differ as their composition diverges from water. For the $225 \mathrm{kV}$ spectrum in phantoms I and II, the larger electron range relative to cavity size increases the influence of the $(\bar{S} / \rho) \underset{\mathrm{m}}{\mathrm{w}}$ term in Equation ( 7$)$. In bony tissue, where $(\bar{S} / \rho)_{\mathrm{m}}^{\mathrm{w}}>1$ (see Figure $5.3(\mathrm{~b})$ ), this contributes to an overall decrease of $D_{\mathrm{mm}}$ in comparison to $D_{\mathrm{wm}}$ (up to - $\mathrm{I} 5 \%$ ). Conversely, for adipose tissue, $(\bar{S} / \rho)_{\mathrm{m}}^{\mathrm{w}}<1$ and there is an increase of $D_{\mathrm{mm}}$ in comparison to $D_{\mathrm{wm}}$ (up to $4.2 \%)$.

For larger cavities, where the range of secondary particles is smaller or comparable to the cavity dimensions, and for lower energies, where the predominance of photoelectric interactions is increased and highly dependent on the tissue-equivalent material compositions, the difference between dose descriptors is much higher. Photoelectric interaction cross sections of bony tissues are higher than those of water, resulting in $D_{\mathrm{mm}}$ values larger than $D_{\mathrm{wm}}$. For spongiosa and cortical bone $D_{\mathrm{mm}}$ ranges from $\mathrm{I.9}$ to I.8 and 2.2 to 4.4 times $D_{\text {wm }}$ for phantoms III and IV, respectively, at 80 $\mathrm{kV}$. The opposite is true for adipose with $D_{\mathrm{mm}} D_{\mathrm{wm}}$ values of 0.76 and 0.69 for phantoms II and IV. 

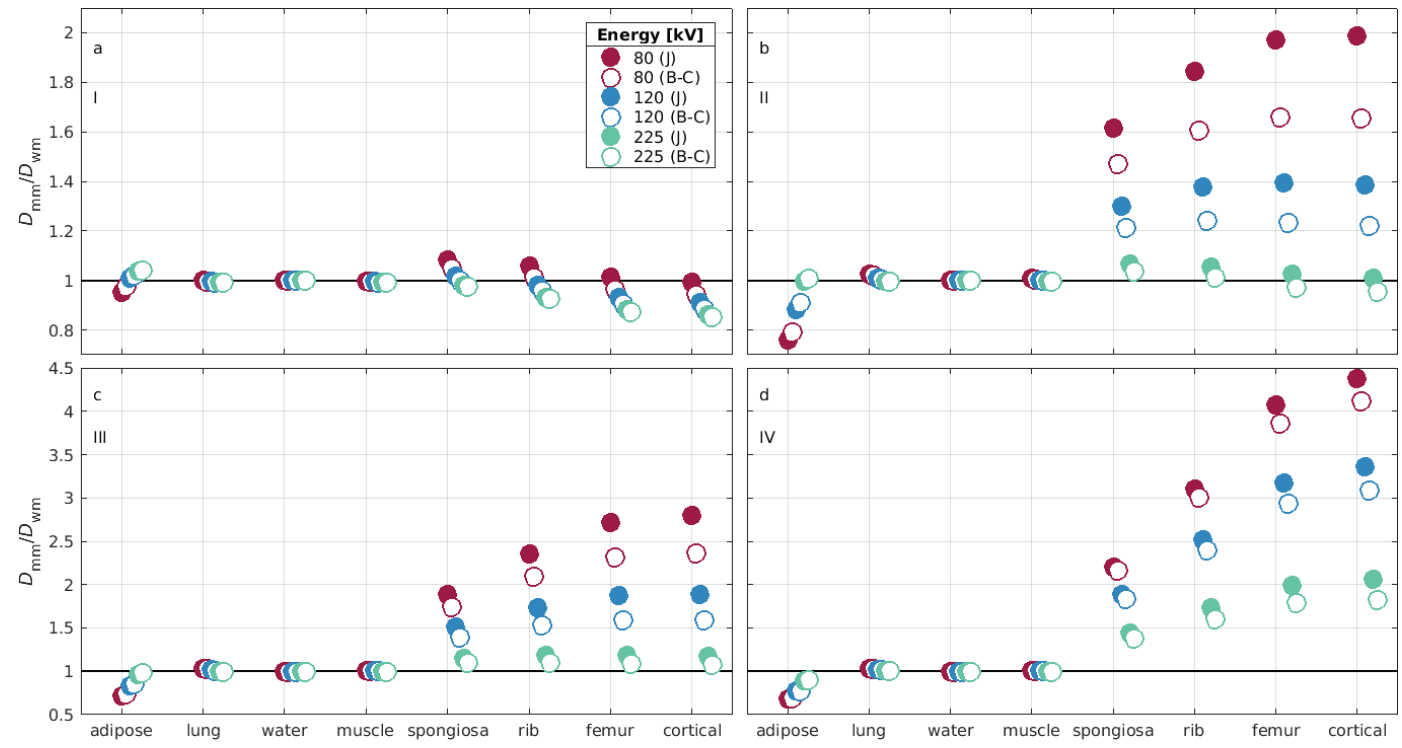

Figure 5.5 - Ratios between the dose descriptors $D_{\mathrm{mm}}$ and $D_{\mathrm{wm}}$ for tissue substitute materials for each phantom size: (a-d) Phantoms I-IV. Each colour represents one of the selected energies: 80 , I20 or $225 \mathrm{kV}$. Full and hollow circles represent $D_{\text {wm }}$ calculated from Janssens (J) and Burlin-Chan (B-C) methods, respectively. The black line highlights cases where $D_{\mathrm{wm}}=D_{\mathrm{mm}}$. The same range of $D_{\mathrm{mm}} / D_{\mathrm{wm}}$ is used for graphs a and b; a different range is used for graphs c and d.

\section{${ }_{5.4 .3} \boldsymbol{D}_{\mathbf{m m}} / \boldsymbol{D}_{\mathbf{w m}}$ : Mouse}

Figure 5.6 presents the ex-vivo male mouse axial and sagittal planes, and the dose map axial views derived from CT scans with either o.I or $0.2 \mathrm{~mm}$ isotropic voxel resolution for the three selected energies. Similar to the phantom calculations, dose differences are higher for lower energies and tissues with photoelectric cross-sections that differ significantly from water. For all scenarios, regions segmented as bony or adipose tissue present the largest difference between dose descriptors. At $225 \mathrm{kV}$, the differences reach maximum and minimum values of $19 \%$ and $-4.0 \%$ (Figure $5.6(\mathrm{f})$ ) or II\% and $-2 \%$, for Burlin-Chan or Janssens' method, respectively, whereas at $80 \mathrm{kV}$ the maximum and minimum differences are $286 \%$ and $-29 \%$ (Figure $5.6(d))$ or $240 \%$ and $-27 \%$. 


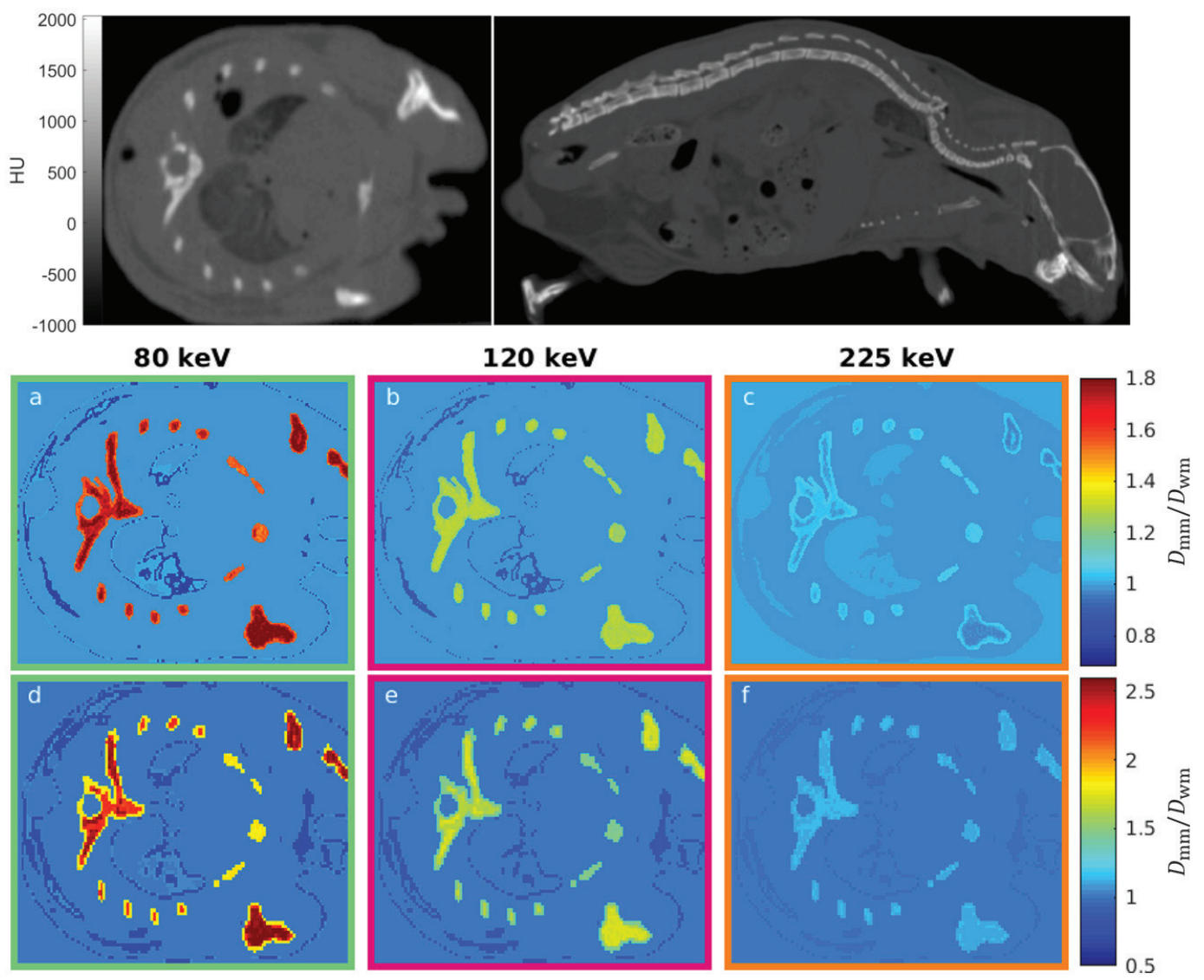

Figure 5.6 - On top: axial (left) and sagittal (right) views of an ex-vivo male mouse CBCT scan of o.I $\mathrm{mm}$ isotropic voxel size. Below: axial view of dose ratio maps simulated from scans with isotropic voxel sizes of $(a-c)$ o.I and $(d-f) 0.2 \mathrm{~mm}$. Simulated irradiation energies of $(a, d) 80,(b, e)$ I20 and $225 \mathrm{kVp}$. The ratios presented were calculated using Burlin-Chan method. Note that although the mouse is fully depicted on the top sagittal view, this study focused on the chest region. 


\subsection{Discussion}

Meaningful pre-clinical radiation research can be translated into successful clinical trials when a deep understanding of the variables that contribute to treatment outcome exists. Accurate dosimetry and comprehension of dose-response mechanisms is paramount. Different from clinical EBRT, small animal radiotherapy requires additional caution for its lower energy range, where differences in dose descriptors are typically magnified. The conversion between dose-to-water and dose-to-medium is fundamentally dependent on the size of cavity. This work used the DOSXYZnre MC simulation code, in which voxel dimension defines the maximum resolution of a dose calculation, regardless of the scale of physical processes contributing to $\mathrm{RBE}^{[8]}$.

A phantom of unique size and composition was subsampled into four different isotropic voxel matrices while maintaining the composition. While the voxel sizes in phantoms II and III are typical of pre-clinical CBCT imaging resolution, those in phantoms I and IV tested extreme scenarios and would be otherwise impractical in terms of calculation times and proper small animal segmentation. The usage of intermediate cavity theory throughout this study demonstrated the influence of small and large cavity theories in the relationship between dose descriptors. Figures 5.3 and 5.4 show the influence of the $\left(\overline{S_{\mathrm{el}}} / \rho\right)_{\mathrm{m}}^{\mathrm{w}},\left(\overline{\mu_{\mathrm{en}}} / \rho\right)_{\mathrm{m}}^{\mathrm{w}}$ or their weighted sum on the dose to the water cavity. Using a constant $\Delta=\mathrm{I} \mathrm{keV}$, user-defined mean chord lengths derived from the voxel dimensions through equation (9) in a range of kilovolt energies. Larger cavities presented lower d values, consequently dominated by the LC term, $\left(\overline{\mu_{\mathrm{en}}} \rho\right)_{\mathrm{m}}^{\mathrm{w}}$ while the inverse was true for small cavities, where the SC term, $\left(\overline{S_{\mathrm{el}}} / \rho\right)_{\mathrm{m}}^{\mathrm{w}}$, dominated. Additionally, considering that $\mathrm{d}$ values vary monotonically with energy, for higher energies the prevalence shifts towards the SC component as secondary particle ranges are increased and tend not to perturb the cavity, favouring $\delta$-ray equilibrium and allowing for the $\left(\overline{S_{\mathrm{el}}} / \rho\right)_{\mathrm{m}}^{\mathrm{w}}$ increased weight.

As cavity dimensions expand, the calculated $D_{\mathrm{mm}} / D_{\mathrm{wm}}$ results shift from values closer to those in Figure 5.5(a) to the ones in Figure 5.5(d). The relationship between dose descriptors is equal or close to unity for all different scenarios for tissues with similar atomic composition and density to water (Figure 5.2(d)). For higher energies, the larger electron range relative to cavity size increases the influence of the $\left(\overline{\mathrm{el}_{\mathrm{e}}} / \rho\right)_{\mathrm{m}}^{\mathrm{w}}$ term in Equation $(7)$. In bony tissue, where $(\bar{S} / \rho)_{\mathrm{m}}^{\mathrm{w}}>1$ this contributes to an overall decrease of $D_{\mathrm{mm}}$ in comparison to $D_{\text {wm }}$, while in adipose tissue, where $(\bar{S} / \rho)_{\mathrm{m}}^{\mathrm{w}}<1$, there is an 
increase of $D_{\mathrm{mm}}$ in comparison to $D_{\mathrm{wm}}$. For larger cavities, where the range of secondary particles is smaller or comparable to the cavity dimensions, and lower energies, where the predominance of photoelectric interactions is increased and highly dependent on the medium composition, the difference between dose descriptors is enhanced. Hence, the impact of the cavity dimensions on estimated $D_{\text {wm }}$ is significant on pre-clinical kilovolt beams. Local spectrum and mean energy were compared to assess if regardless of localization, the conversion factor would remain unique; this consideration is only relevant for materials that differ from water. Differences up to 5.I\% were found between $D_{\mathrm{mm}} / D_{\text {wm }}$ ratios calculated using the local spectrum on different regions within the mouse. This is a minor effect, possibly negligible when the uncertainty on the underlying tissue composition is considered. It also points to a minor hardening of the beam (the selective attenuation of low energy photons) within the small specimens.

Caution is advised when using the IC theory as there is a lack of consensus in the literature on d-parameter deriving methods. Besides the two presented here, Burlin-Chan and Janssens theories, several others exist ${ }^{[16,28,29]}$ and may be responsible for further discrepancies between dose descriptors. This study presented a tool to investigate the effect of distinct $\mathrm{d}$-values in CT phantoms. For the two investigated methods, $D_{w m}$ in bony tissue differed by up to $20 \%$ for the same cavity size and irradiation energy, while for water-like tissues, differences were negligible. Mobit et al. used MC to track the dose fraction of electrons arising in the cavity. Tedgren and Carlsson presented three methods for calculating $d$, including equations for determining the effective mass absorption coefficient of electron fluence penetrating the cavity $(\beta)$. Oliver and Thomson showed five different approaches to calculating $\mathrm{d}$ and found that agreement between ICT and MC-computed values of $D_{\text {wm }}$ was best when Figure 5.I (c), equation (8) was used with $\beta$ calculated using Janssens' method.

Janssens theory is more rigorous: while $\beta$ was somewhat arbitrarily chosen by Burlin-Chan, Janssens calculated the attenuation and energy distribution of secondary particles including energy degradation due to the slowing down of the electrons in the cavity and considered electron backscatter at the interface between different media ${ }^{[41]}$, which is possibly the culprit for consistently higher $d$ values. Further simulations and measurements, explicitly scoring secondary particles are recommended for establishing a more generally accepted ICT, and MC calculation of dose in an embedded water cavity could bring more definitive answers. Additionally, for both methods used in this study, the maximum range of primary electrons was 
taken as CSDA values from the ICRU Report $9 \mathrm{O}^{\left[3_{6} 6\right.}$. This approximation is an over-estimate as electron paths are not straight lines. Range values can also be calculated using the cross-section database of the MC code. For EGSnrc codes, cross-section data is typically generated using the restricted stopping power for dose calculations. However, for range estimations, cross-section data should be generated with the total stopping power. This is stressed here as it may cause confusion and impact on $\mathrm{d}$ calculations.

The uncertainty over a meaningful cavity dimension should also be considered. Besides the conversion between analytical methods and MBDC calculations, cavity theory and conversion factors are also relevant for the biological dose deposition. Several works ${ }^{[8,10,16,18,42]}$ have discussed the water cavity dimensions' significance with respect to cellular structures and biological effects, and some have attempted to ascertain links to biological effectiveness, for example, via comparison with the DNA-bound water molecules. However, the biological unknowns, e.g. the influence of repair mechanisms and composition and geometry of target surrounding structures, add another level of complexity, often only approached by micro-dosimetry techniques.

Beyond cavity size, improved imaging capabilities, in terms of quality and optimized protocols, is fundamental: incorrect tissue assignment can lead to differences between dose descriptors. Although a necessary MC calculation step, CT number to medium conversion is also a source of uncertainty due to limitations intrinsic to CT scanners, conversion functions, number of materials and lack of pre-clinical specific data. It is common ${ }^{[37]}$ to assign human tissue compositions ${ }^{\left[3^{8,43}\right]}$ to small animals due to the absence of specific data. This practice is somewhat supported by recent studies on human and mouse tissue compositions as measured by dual-energy CT imaging ${ }^{[39]}$. Nevertheless, it has been shown that mean excitation energy variation has a significant impact on $\left(\bar{S}_{\mathrm{el}} / \rho\right)_{\mathrm{m}}^{\mathrm{w}}{ }^{[44]}$.

Extensive experimental evidence is another fundamental, yet still lacking, aspect of this field. Correlation between dose descriptors and treatment outcome could potentially corroborate the choice of one dose descriptor over the other. For the ex-vivo male mouse used in this study, dose differences from $-29 \%$ to $286 \%$ were found (Figure 5.6). Currently, pre-clinical research technology, in terms of precise targeted irradiation coupled to on-board imaging devices and advanced biological models, would allow the design of measurement studies, not feasible before. Nevertheless, as long as radiotherapy dosimetry is based on these descriptors, dose distribution comparisons should be performed with the same reporting quantity and 
caution regarding reported quantity and dose comparisons with preclinical research or the clinical standard is advised.

\subsection{Conclusion}

Pre-clinical radiotherapy dose calculations using detailed on-the-fly MC simulations for ICT were presented for a range of voxel sizes (cavities), irradiation energies and $\mathrm{d}$-parameter calculation methods. The MC code modifications facilitated rapid investigation of CT phantoms. ICT made the influence of $\left(\overline{S_{\mathrm{el}}} / \rho\right)_{\mathrm{m}}^{\mathrm{w}}$ and $\left(\overline{\mu_{\mathrm{en}}} / \rho\right)_{\mathrm{m}}^{\mathrm{w}}$ explicit through the d-parameter: smaller cavities presented higher $\mathrm{d}$ values, consequently dominated by the SCT term, the $\left(\overline{S_{\mathrm{el}}} / \rho\right)_{\mathrm{m}}^{\mathrm{w}}$, with the inverse being true for LCT and $\left[\mu_{\mathrm{en}} / \rho\right]_{\mathrm{m}}^{\mathrm{w}}$ The impact of the cavity dimensions on estimated $D_{\mathrm{wm}}$ is significant on preclinical kilovolt beams. At lower energies, for tissues with the elemental compositions most divergent from water, e.g. bony and fatty tissues, the $D_{\mathrm{mm}} / D_{\mathrm{wm}}$ ranged between 0.68 and 4.4 . This result can directly impact preclinical research translation as such differences are not present in MV clinical radiotherapy. Moreover, biological effects on bony tissues may affect the irradiated subjects. From the results of this study, caution is advised when using the ICT due to a lack of consensus on d-parameter deriving methods. Dose computed using Burlin-Chan or Janssens methods for the same geometry, energy and cavity size differed by up to $20 \%$ for osseous tissues. Although Janssens' method is more rigorous, further simulations and measurements, explicitly scoring secondary particles, are recommended for establishing a more generally accepted ICT, and an explicit MC calculation of dose in an embedded water cavity could bring definite answers. For a heterogenous geometry, the ex-vivo mouse specimen, differences in dose descriptors of up to $286 \%$ were found. This result points to an important missing piece of information: the need of measurement-based results, which may correlate dose descriptor and biological outcome within a living organism represent. The advanced small animal precision radiotherapy field is likely to provide the ideal platform for such experiments. Finally, dose distribution comparisons should use the same reporting quantity and caution regarding reported quantity and dose comparisons is advised. 


\section{AI Appendix AI}

\section{AI.I Local photon spectra}

The local photon spectra were generated, using the MC code MCNP6 (Monte Carlo n-Particle I.o) ${ }^{[45]}$, as differences between $D_{\mathrm{mm}}$ and $D_{\mathrm{wm}}$ may be dependent on local photon energy fluence spectrum, which varies throughout the geometry ${ }^{\left[r_{4}\right]}$. An additional aim was to evaluate beam hardening effects (the selective attenuation of low energy photons). Three irradiation spectra were considered as $\left[\mu_{\mathrm{en}} / \rho\right]_{\mathrm{m}}^{\mathrm{w}}$ varies with energy and fifteen regions were selected in the mouse CT scan comprising different tissues and positions. A sufficient number of histories $\left(\mathrm{I}^{-} 4^{\cdot} \mathrm{IO}^{9}\right)$ was used to achieve an overall statistical uncertainty of $0.5 \%$ MCNP6 was used because it was not possible to score local photon spectra with the version of DOSXYZnrc used in this study. Nevertheless, the codes were benchmarked against each other using the same datasets and provided excellent agreement $\left(D_{\mathrm{mm}}\right.$ differences on the order of the statistical noise) - data is not shown for sake of brevity. Figure 5.AI(a) shows the points where local photon spectra were generated. They are located in heterogeneous regions, e.g. Pio-I4, and uniform ones, e.g. $\mathrm{P}_{7}, \mathrm{P}_{15}$. The shape of the spectra, Figures $5 . \mathrm{A}_{\mathrm{r}}(\mathrm{b}-\mathrm{d})$, remained similar in all regions, with prevailing variations in intensity. As the radiation beam impinged on the specimen from the bottom, intensity variation throughout the geometry is expected. $\mathrm{P}_{2}$ and $\mathrm{P}_{7}$, the farthermost points, presented the maximum integral (under the curve) differences of $4 \mathrm{I} \%, 38 \%$ and $32 \%$ for 80 , I20 and $225 \mathrm{kV}$, respectively. $D_{\mathrm{mm}} D_{\mathrm{wm}}$ ratios were taken at these two points using the local spectrum and substituting the local medium by cortical bone or water. Differences of $2.3 \%, 3.5 \%$ and $5.1 \%$ were identified between the $D_{\mathrm{mm}} / D_{\text {wm }}$ ratios of $\mathrm{P}_{2}$ and $\mathrm{P}_{7}$ for 80, I20 and $225 \mathrm{kV}$. Furthermore, a decrease in the mean spectral energy of $2.1 \%, 2.2 \%$ and $3.0 \%$ was observed between $\mathrm{P}_{2}$ and $\mathrm{P}_{7}$ for 80 , I20 and $225 \mathrm{kV}$. 

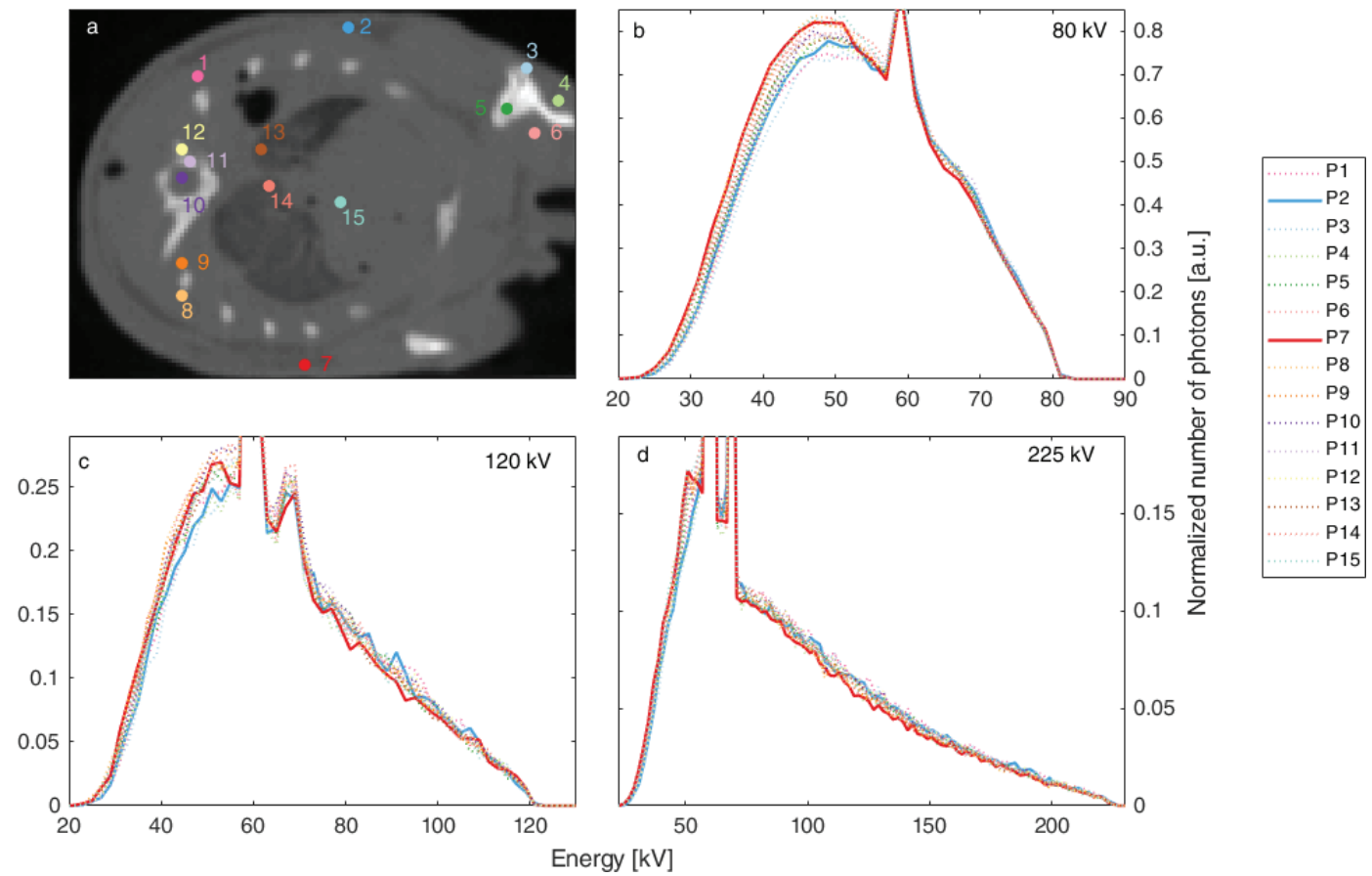

Figure 5.AI - (a) The numbers I to 15 indicate the points where the local photon spectra were generated for the ex vivo mouse CT scan with isotropic voxel sizes of $0.2 \mathrm{~mm}$. b-d) Local photon spectra for each of the fifteen points from Figure 5.AI(a) for calculations using 80, I20 and 225 $\mathrm{kV}$ photons. They were grouped in energy bins of 2,3 and $4 \mathrm{kV}$, respectively. The solid lines represent points 2 and $7\left(\mathrm{P}_{2}\right.$ and $\left.\mathrm{P}_{7}\right)$. The dotted lines represent the remaining points, illustrating the spectral shape conservation throughout the geometry. The spectra were normalized by the maximum photon intensity for each point and the bremsstrahlung region is in evidence, while the characteristic peaks are outside the limits of Figures $(b-d)$, to stress inter-spectral differences.

For small animal irradiations the differences between $D_{\mathrm{mm}} / D_{\mathrm{wm}}$ associated with local photon energy fluence spectrum are minor, in comparison to clinical $\mathrm{kV}$ treatments due to much smaller geometries and treatment beams - generally, the cross-section of a mouse does not exceed $3 \mathrm{~cm}$ and even in a rat the difference would be small. 


\subsection{References}

${ }^{[1]}$ I.J. Chetty, B. Curran, J.E. Cygler, J.J. DeMarco, G. Ezzell, B.A. Faddegon, I. Kawrakow, P.J. Keall, H. Liu, C.M. Ma, D.W. Rogers, J. Seuntjens, D. Sheikh-Bagheri, J.V. Siebers, Report of the AAPM Task Group No. I05: Issues associated with clinical implementation of Monte Carlo-based photon and electron external beam treatment planning, Med Phys, 34 (2007) 48I8-4853.

${ }^{[2]}$ G. Landry, B. Reniers, J.P. Pignol, L. Beaulieu, F. Verhaegen, The difference of scoring dose to water or tissues in Monte Carlo dose calculations for low energy brachytherapy photon sources, Med Phys, 38 (2011) I526-I533.

${ }_{33}$ H.H. Liu, P. Keall, W.R. Hendee, Dm rather than Dw should be used in Monte Carlo treatment planning, Medical Physics, 29 (2002) 922-924.

${ }^{[4]}$ P. Andreo, Dose to 'water-like' media or dose to tissue in MV photons radiotherapy treatment planning: still a matter of debate, Phys Med Biol, 6o (2015) 309-337.

${ }^{[5]}$ B.R. Walters, R. Kramer, I. Kawrakow, Dose to medium versus dose to water as an estimator of dose to sensitive skeletal tissue, Phys Med Biol, 55 (2010) 4535-4546.

${ }^{[6]}$ J.V. Siebers, P.J. Keall, A.E. Nahum, R. Mohan, Converting absorbed dose to medium to absorbed dose to water for Monte Carlo based photon beam dose calculations, Physics in Medicine and Biology, 45 (2000) 983-995.

${ }^{[7]}$ N. Dogan, J.V. Siebers, P.J. Keall, Clinical comparison of head and neck and prostate IMRT plans using absorbed dose to medium and absorbed dose to water, Phys Med Biol, 5I (2006) 4967-4980.

${ }^{[8]}$ B.R. Walters, Application of efficient Monte Carlo photon beam simulations to dose calculations in voxellized human phantoms, Physics, University of Maastricht, Maastricht, The Netherlands, 20I7.

${ }_{99}$ B.R. Walters, G.X. Ding, R. Kramer, I. Kawrakow, Skeletal dosimetry in cone beam computed tomography, Med Phys, 36 (2009) 2915-2922.

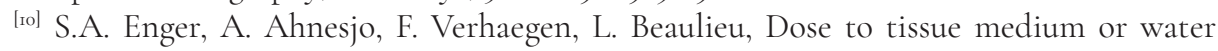
cavities as surrogate for the dose to cell nuclei at brachytherapy photon energies, Phys Med Biol, 57 (2012) 4489-4500.

${ }^{[r]}$ F. Verhaegen, P. Granton, E. Tryggestad, Small animal radiotherapy research platforms, Phys Med Biol, 56 (2011) R55-83.

${ }^{\left[{ }_{2}\right]}$ F. Verhaegen, S. van Hoof, P.V. Granton, D. Trani, A review of treatment planning for precision image-guided photon beam pre-clinical animal radiation studies, Z Med Phys, 24 (2014) 323-334.

${ }^{\left[{ }_{3}\right]}$ K.T. Butterworth, K.M. Prise, F. Verhaegen, Small animal image-guided radiotherapy: status, considerations and potential for translational impact, $\mathrm{Br} \mathrm{J}$ Radiol, 88 (2015) 20140634 .

${ }^{\left[{ }_{4}\right]}$ G.P. Fonseca, A.C. Tedgren, B. Reniers, J. Nilsson, M. Persson, H. Yoriyaz, F. Verhaegen, Dose specification for I92Ir high dose rate brachytherapy in terms of dose-to-water-inmedium and dose-to-medium-in-medium, Phys Med Biol, 6o (2015) 4565-4579.

${ }^{\left.{ }_{55}\right]}$ F. Verhaegen, Chapter 20. Treatment Planning for Small Animals, in: G.C. Kagadis, N.L. Ford, D.N. Karnabatidis, G.K. Loudos (Eds.) Chapter 20. Treatment Planning for Small Animals; Handbook of Small Animal Imaging: Preclinical Imaging, Therapy, and Applications, CRC Press, Boca Raton, FL, 2016, pp. 6II. 
${ }^{[16]}$ A.C. Tedgren, G.A. Carlsson, Specification of absorbed dose to water using modelbased dose calculation algorithms for treatment planning in brachytherapy, Phys Med Biol, 58 (2013) 256I-2579.

${ }^{\left[{ }_{1}\right]}$ P.A.K. Oliver, R.M. Thomson, Cavity theory applications for kilovoltage cellular dosimetry, Phys Med Biol, 62 (2017) 4440-4459.

${ }^{[18]}$ D.T. Goodhead, Energy deposition stochastics and track structure: what about the target?, Radiat Prot Dosimetry, I22 (2006) 3-15.

${ }^{\left[{ }_{19}\right]}$ F. Verhaegen, L. Dubois, S. Gianolini, M.A. Hill, C.P. Karger, K. Lauber, K.M. Prise, D. Sarrut, D. Thorwarth, C. Vanhove, B. Vojnovic, R. Weersink, J.J. Wilkens, D. Georg, ESTRO ACROP: Technology for precision small animal radiotherapy research: Optimal use and challenges, Radiother Oncol, I26 (2018) 47I-478.

${ }^{[20]}$ B.R. Walters, I. Kawrakow, D.W. Rogers, DOSXYZnrc Users Manual, NRC Report PIRS-0794, Rev B (2005) I-I25.

${ }^{\left[{ }_{21}\right]}$ M.J. Berger, M. Inokuti, H.H. Anderson, H. Bichsel, J.A. Dennis, D. Powers, S.M. Seltzer, J.E. Turner, Report 37, Journal of the International Commission on Radiation Units and Measurements, osig (I984) NP-NP.

${ }^{\left[{ }_{22}\right]}$ P. Andreo, D.T. Burns, A.E. Nahum, J.P. Seuntjens, F.H. Attix, Fundamentals of Ionizing Radiation Dosimetry, Wiley-VCH, Berlin, 2017.

${ }^{{ }^{23} 3}$ A.E. Nahum, Water/air mass stopping power ratios for megavoltage photon and electron beams, Physics in Medicine and Biology, 23 (1978) 24-38.

${ }^{\left.{ }^{2} 4\right]}$ F.H. Attix, L.D. Vergne, V.H. Ritz, Cavity ionization as a function of wall material, Journal of Research of the National Bureau of Standards, 6o (1958).

${ }^{[25]}$ T.E. Burlin, A general theory of cavity ionisation, Br J Radiol, 39 (1966) 727-734.

${ }^{\left[{ }_{26} 6\right]}$ T.E. Burlin, F.K. Chan, The effect of the wall on the Fricke dosemeter, The International Journal of Applied Radiation and Isotopes, 20 (1969) 767-775.

${ }^{[27]}$ A. Janssens, G. Eggermont, R. Jacobs, G. Thielens, Spectrum perturbation and energy deposition models for stopping power ratio calculations in general cavity theory, Physics in Medicine and Biology, I9 (1974) 619-630.

${ }^{[28]}$ R. Loevinger, The dosimetry of beta sources in tissue; the point-source function, Radiology, 66 (1956) 55-62.

${ }^{[29]}$ P.N. Mobit, A.E. Nahum, P. Mayles, An EGS 4 Monte Carlo examination of general cavity theory, Physics in Medicine and Biology, 42 (1997) I319-I334.

${ }^{{ }^{1} \mathrm{ol}}$ I. Kawrakow, D. Rogers, The EGSnrc code system: Monte Carlo simulation of electron and photon transport, DOI (2000).

${ }^{\left[{ }_{31}\right]}$ D.W.O. Rogers, I. Kawrakow, J. Seuntjens, B.R. Walters, NRC User Codes for EGSnrc, National Research Council of Canada, Ottawa, Canada, Technical Report PIRS-702, DOI (2000).

${ }^{\left[{ }_{3} 2\right]}$ R. Clarkson, P.E. Lindsay, S. Ansell, G. Wilson, S. Jelveh, R.P. Hill, D.A. Jaffray, Characterization of image quality and image-guidance performance of a preclinical microirradiator, Med Phys, 38 (20II) 845-856.

${ }_{333}$ P.V. Granton, F. Verhaegen, On the use of an analytic source model for dose calculations in precision image-guided small animal radiotherapy, Phys Med Biol, 58 (2013) 3377-3395.

${ }^{\left.{ }_{344}\right]}$ G.G. Poludniowski, Calculation of x-ray spectra emerging from an x-ray tube. Part II. X-ray production and filtration in x-ray targets, Med Phys, 34 (2007) 2175-2186.

${ }^{\left.{ }_{35}\right]}$ G. Poludniowski, G. Landry, F. DeBlois, P.M. Evans, F. Verhaegen, SpekCalc: a program to calculate photon spectra from tungsten anode x-ray tubes, Phys Med Biol, 54 (2009) $\mathrm{N}_{433}-438$. 
[36] Report 90 - Key data for ionizing-radiation dosimetry: measurement standards and applications, Journal of the International Commission on Radiation Units and Measurements (ICRU), I4 (2016) IIo.

${ }^{[37]}$ A. Vaniqui, L. Schyns, I.P. Almeida, B. van der Heyden, S.J. van Hoof, F. Verhaegen, The impact of dual energy CT imaging on dose calculations for pre-clinical studies, Radiat Oncol, I2 (2017) I8I.

${ }^{[38]}$ D.R. White, J. Booz, R.V. Griffith, J.J. Spokas, I.J. Wilson, Report 44, Journal of the International Commission on Radiation Units and Measurements, os23 (I989) NP-NP.

${ }^{\left.{ }^{3}{ }^{2}\right]}$ L.E. Schyns, D.B. Eekers, B. van der Heyden, I.P. Almeida, A. Vaniqui, F. Verhaegen, Murine vs human tissue compositions: implications of using human tissue compositions for photon energy absorption in mice, Br J Radiol, DOI Io.I259/bjr.20I80454(20I8) 20180454. ${ }^{[40]}$ C.D. Johnstone, P. Lindsay, E.E. Graves, E. Wong, J.R. Perez, Y. Poirier, Y. Ben-Bouchta, T. Kanesalingam, H. Chen, A.E. Rubinstein, K. Sheng, M. Bazalova-Carter, Multiinstitutional MicroCT image comparison of image-guided small animal irradiators, Phys Med Biol, 62 (2017) 5760-5776.

${ }^{[41]}$ A. Janssens, The fundamental constraint of cavity theory, Physics in Medicine and Biology, 29 (I984) II57-II58.

${ }^{\left[{ }_{42}\right]}$ R.M. Thomson, A.C. Tedgren, J.F. Williamson, On the biological basis for competing macroscopic dose descriptors for kilovoltage dosimetry: cellular dosimetry for brachytherapy and diagnostic radiology, Phys Med Biol, 58 (2013) II23-II50.

${ }^{\left.{ }^{143}\right]}$ ICRP, Report of the Task Group on Reference Man, Pergamon Press, Oxford., ICRP Publication 23 (I975).

${ }^{\left[{ }_{44}\right]}$ P. Andreo, D.T. Burns, F. Salvat, On the uncertainties of photon mass energy-absorption coefficients and their ratios for radiation dosimetry, Phys Med Biol, 57 (2012) 2II7-2I36.

${ }^{\left.{ }_{445}\right]}$ T. Goorley, M. James, T. Booth, F. Brown, J. Bull, L.J. Cox, J. Durkee, J. Elson, M. Fensin, R.A. Forster, J. Hendricks, H.G. Hughes, R. Johns, B. Kiedrowski, R. Martz, S. Mashnik, G. McKinney, D. Pelowitz, R. Prael, J. Sweezy, L. Waters, T. Wilcox, T. Zukaitis, Initial MCNP6 Release Overview, Nuclear Technology, I80 (2012) 298-315. 



\section{CHAPTER 6}

Exploring the feasibility of a clinical proton beam with an adaptive aperture for pre-clinical research

Isabel P Almeida, Ana Vaniqui, Lotte EJR Schyns, Brent van der Heyden, James Cooley, Townsend Zwart, Armin Langenegger, Frank Verhaegen

British Journal of Radiology

November 2018, 92(1095), 20180447

British Journal of Radiology 


\section{Abstract}

Objectives: To investigate whether the Mevion S25oi with HYPERSCAN clinical proton system could be used for pre-clinical research with millimetric beams.

Methods: The nozzle of the proton beam line, consisting of an energy modulation system (EMS) and an adaptive aperture (AA), was modelled with the TOPAS Monte Carlo (MC) Simulation Toolkit. With the EMS, the $230 \mathrm{MeV}$ beam nominal range can be decreased in multiples of $2.1 \mathrm{~mm}$. MC dose calculations were performed in a mouse lung tumour CT image.

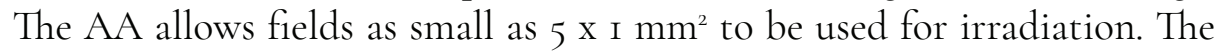
best plans to give 2 Gy to the tumour were derived from a set of discrete energies allowed by the EMS, different field sizes and beam directions. The final proton plans were compared to a precision photon irradiation plan. Treatment times were also assessed.

Results: Seven different proton beam plans were investigated, with a good coverage to the tumour (D95>I.95 Gy, D5<2.3 Gy) and with potentially less damage to the organs at risk (OARs) than the photon plan. For very small fields and low energies, the number of protons arriving to the target drops to $\mathrm{I}-3 \%$, nevertheless the treatment times would be below 5 seconds.

Conclusions: The proton plans made in this study, collimated by an AA, could be used for animal irradiation.

Advances in knowledge: This is one of the first study to demonstrate the feasibility of pre-clinical research with a clinical proton beam with an adaptive aperture used to create small fields. 


\subsection{Introduction}

The number of ion beam therapy centers has been growing rapidly in the last decade, making ion beam therapy a possible cancer treatment. To advance the understanding of ion beam therapy, pre-clinical research is needed combining different tumour models and ion beam irradiation ${ }^{[\mathrm{r}]}$.

Also in recent years, specialized image guided irradiation platforms for conventional photon radiotherapy were installed in over one hundred research departments worldwide, an order of magnitude more than in $20{ }^{[2]}$. Such platforms are radically changing the field of pre-clinical animal research in radiobiology by using image guided irradiation with milimetric beams ${ }^{[3]}$. Furthermore, specific treatment planning systems were developed, where the physics of low-energy $\mathrm{x}$-ray beams was modelled to deliver accurately the prescribed dose to the target, while considering the constraints in dose to the organs-at-risk (OARs) ${ }^{[4-6]}$.

Integrating the existing state-of-the-art photon platforms with ion beams will initiate a completely new field in pre-clinical research. Greubel et al ${ }^{[7]}$ presented a study where 24 subcutaneous tumours in mice were irradiated with 20 Gy using $23 \mathrm{MeV}$ proton beams and tumour growth was evaluated. Moreover, Constanzo et a ${ }^{[8]}$ published a radiobiological study, where a 4 $\mathrm{MeV}$ mini-proton beam was used to irradiate tumour cells and survival curves were comparable to the ones obtained with $\mathrm{x}$-ray irradiation. In 2017, Ford et al ${ }^{[9]}$ demonstrated the feasibility of integrating a $50 \mathrm{MeV}$ proton beam with a commercially available $\mathrm{x}$-ray pre-clinical platform. The same group developed a Monte Carlo (MC) model for a small animal proton beamline and investigated the relative biological effectiveness (RBE) in tissues using fractionated proton minibeams ${ }^{[\mathrm{ro}]}$.

Since it is unlikely that dedicated small animal ion beam therapy facilities will be built due to cost, current clinical and non-clinical ion beam facilities look for solutions to adapt their beams for image guided pre-clinical research ${ }^{\left[\mathrm{In}-\mathrm{r}_{3}\right]}$, while making use of platforms with existing $\mathrm{x}$-ray beams for imaging and positioning ${ }^{[2]}$. For this reason, the work here presented investigates the possible use of a clinical proton beam, which has a unique adaptive aperture to create small fields with sharp beam penumbras and low proton energies, for small animal research. The study will focus on dose calculations with proton beam, and will not consider any effects of relative biological effectiveness at low proton energies. This would require extensive studies of the RBE in small proton beams in in vivo systems. 


\subsection{Methods and materials}

\subsection{Clinical system}

The MEVION S25oi with HYPERSCAN proton system (Mevion Medical Systems Inc., Littleton, Ma, USA) is a commercially available one-room compact system with a synchrocyclotron proton accelerator mounted on the gantry, which rotates in the treatment room around the patient (figure 6.ra). The beam line consists of a dual axis scanning magnet, transmission ion chambers, a fast energy modulation system (EMS) and an Adaptive Aperture TM (AA) for beam collimation (figure 6.Ib). These components are mounted on the Nozzle, that can rotate between -5 and 185 degrees in the gantry plane (figure 6.ra). Furthermore, the module that contains the EMS and AA can be extended towards the isocenter, ranging from 3.6 to $33.6 \mathrm{~cm}$.

The system produces a pencil beam with a fixed energy of $230 \mathrm{MeV}$ and uses the EMS to decrease the pencil beam energy. This device consists of eighteen Lexan plates with specific thicknesses and air gaps that allow for a decrease of the pencil beam residual range in multiples of $0.2 \mathrm{Im}$ in water, covering a depth from $32 \mathrm{~cm}$ (90\% distal fall-off) to the surface. The AA is located at the end of the beam line and it consists of two opposing blocks of seven leaves made of $\mathrm{Nickel}^{[\mathrm{r}]]}$ moving in a $20 x 2 \mathrm{~cm}^{2}$ treatment field enabling collimation of each pencil beam per energy layer. The AA moves as a block in the $y$-direction and each leaf can move in the $\mathrm{x}$-axis direction (figure 6.Ib,c). Furthermore, the one nominal proton energy combined with the type of EMS, results in Bragg Peaks with a constant range straggling (the full-width half maximum (FWHM) in the beam direction of approximately $27 \pm \mathrm{Imm}$ measured in integrated depth doses (IDDs)).

\subsubsection{Beam model}

The beam model of the S250i with HYPERSCAN system was created using the MC platform TOolkit for PArticle Simulation (TOPAS) ${ }^{[5]}$ (figure 6.Ic). The model was validated with data provided by the manufacturer. 

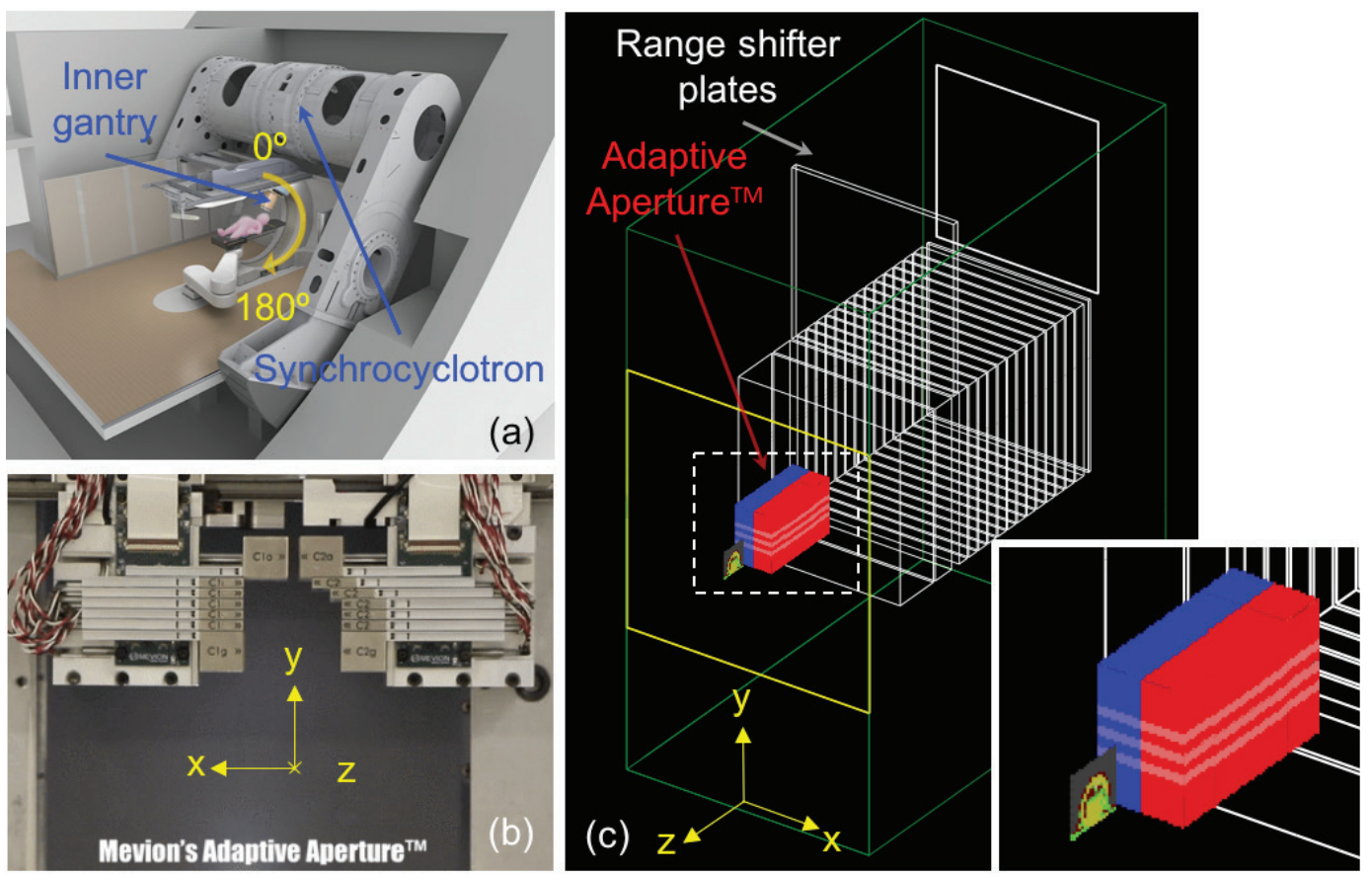

Figure 6.I. (a) treatment room sketch with the synchrocyclotron mounted on the gantry, which rotates around the isocentre from -5 to 185 degrees; (b) close view of the adaptive aperture (AA) module in the beam's-eye-view; (c) MC model of the module with the I6 plates of Energy Modulation System (EMS) in the beam path, indicating the range shifter plates, the AA and the exit window (the beam goes from right to left) with a representative slice of the micro cone-beam CT of the mouse in front of the nozzle. The dotted box is zoomed-in on the right.

\subsubsection{Simulation set-up}

The submillimetric cone beam computed tomography $(\mu \mathrm{CBCT})$ image of a mouse with an orthotopic lung tumour was acquired with the X-RAD $225 \mathrm{Cx}$ system (Precision X-Ray, North Branford, CT, USA) at a tube voltage of 80 $\mathrm{kVp}$ and with a o.I x o.I x O.I $\mathrm{mm}^{3}$ voxel size. The main structures (tumour in the right lung, right and left lungs, spinal cord and heart) were delineated by a radiation oncologist. Using a dedicated small animal radiotherapy planning system SmART-Plan ${ }^{[6]}$ (research version I.5, Precision X-Ray) an optimal photon irradiation plan was designed to administer a prescribed dose of 2 Gy to the tumour (figure 6.2a). 


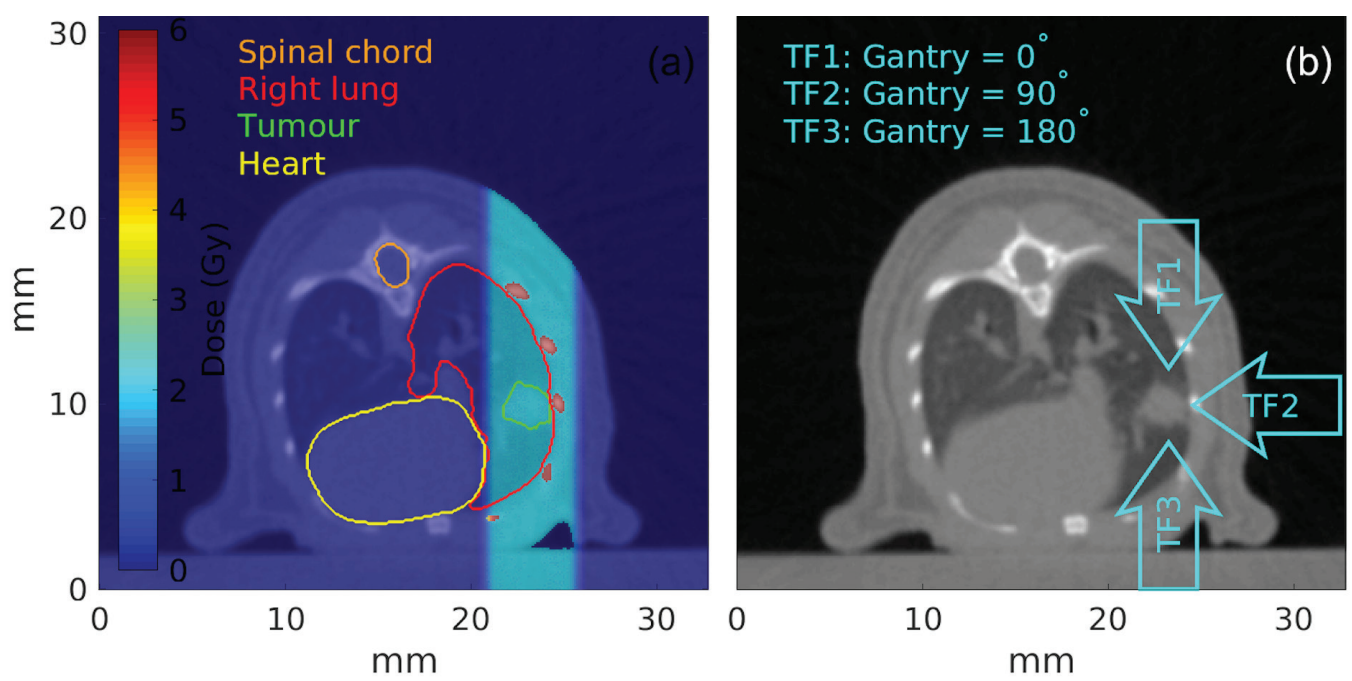

Figure 6.2. (a) Photon dose distribution, micro cone-beam CT image and structure contours for the photon plan with two opposed $225 \mathrm{kVp}$ photon beams using a $5 \mathrm{~mm}$ circular collimator to deliver a prescribed dose of 2 Gy to the tumour (statistical uncertainty of o.I\%, equivalent to approximately Io 8 histories per beam). The tumour is indicated in green in the right lung. (b) Illustration of the three proton treatment fields (and corresponding inner gantry rotation) used to create seven different proton plans.

For the proton plans, simulations of a single spot proton pencil beam aimed at the tumour centre were performed combining two or three treatment fields (TF), using different energies and five field sizes (table 6.I, figure $6.2 b)$. The choice of energy was limited by the EMS discrete steps. Treatment scenarios Ti to T6 use treatment fields with low energies (29.I4 MeV, 32.97 $\mathrm{MeV}, 36.46 \mathrm{MeV}$ and $39.72 \mathrm{MeV}$ ), in which the Bragg Peak stops inside the tumour, while $\mathrm{T}_{7}$ uses two opposed $158.87 \mathrm{MeV}$ beams to 'shoot-through' the tumour. The minimum field size in the y-direction (axis in figure 6.Ib) is limited by the leaf thickness of $5 \mathrm{~mm}$, however in the $\mathrm{x}$-direction, any opening is possible with $0.5 \mathrm{~mm}$ resolution. The default physics list from TOPAS, validated for proton beams ${ }^{[9,16]}$, was used and dose-to-water-inmedium (physical dose) was scored in a $0.2 \times 0.2 \times 0.2 \mathrm{~mm}^{3}$ grid (double the original $\mu \mathrm{CBCT}$ voxel size). A primary $230 \mathrm{MeV}$ proton beam with an energy spread (standard deviation) of $0.2174 \mathrm{MeV}$ and a virtual source-axisdistance (SAD) of $\mathrm{I} 85 \mathrm{~cm}$, was simulated with $\mathrm{I} .75 \times \mathrm{IO}^{9}$ protons per TF. 


\begin{tabular}{|c|c|c|c|c|}
\hline \multirow{2}{*}{$\begin{array}{l}\text { Treatment } \\
\text { scenario \# }\end{array}$} & \multicolumn{3}{|c|}{ Treatment fields (MeV) } & \multirow{2}{*}{$\begin{array}{l}\text { Field size } \\
\left(\mathrm{mm}^{2}\right)\end{array}$} \\
\hline & TF 1 & TF 2 & TF 3 & \\
\hline TI & $39 \cdot 72$ & 29.14 & 36.46 & \multirow[b]{2}{*}{$5 \times 2.5$} \\
\hline $\mathrm{T}_{2}$ & $39 \cdot 72$ & 32.97 & 39.72 & \\
\hline $\mathrm{T}_{3}$ & $39 \cdot 72$ & 29.14 & 36.46 & \multirow{2}{*}{$5 \times 3$} \\
\hline $\mathrm{T}_{4}$ & $39 \cdot 72$ & 32.97 & 39.72 & \\
\hline $\mathrm{T}_{5}$ & $39 \cdot 72$ & - & 36.46 & \multirow[t]{2}{*}{$5 \times 3.5$} \\
\hline $\mathrm{T} 6$ & $39 \cdot 72$ & - & 39.72 & \\
\hline $\mathrm{T}_{7}$ shoot-through & I58.87 & - & I58.87 & $5 \times 4$ \\
\hline
\end{tabular}

Table 6.I. Simulation set-up for 7 different treatment scenarios (T), using two or three treatment fields $\left(\mathrm{TF} I-\mathrm{TF}_{3}\right)$ defined by the gantry rotation $\mathrm{o}^{\circ}, 90^{\circ}$ and $\mathrm{I}_{80^{\circ}}$ (explicitly in figure 6.ra), respectively. The last column shows the four different field sizes created with the adaptive aperture for each TF.

Each treatment scenario resulted in $64\left(\mathrm{~T}_{\mathrm{I}}-\mathrm{T}_{4}\right)$ or $\mathrm{I}_{6}\left(\mathrm{~T}_{5}-\mathrm{T}_{7}\right)$ dose distributions (table 6.2), for each of these the dose volume histogram (DVH) metrics $\mathrm{D}_{95}$ and $\mathrm{D}_{5}$ (dose given to $95 \%$ and $5 \%$ of the tumour volume, respectively) were calculated to select the best plan for each treatment. Furthermore, the DVH metrics V9o and Vio were used to evaluate the volume of OARs that received 90\% and 10\% of the prescribed dose, respectively. Finally, the best dose distributions from the seven different scenarios were compared, arriving at the plan that best covers the tumour with the prescribed dose. 


\begin{tabular}{|c|c|c|c|c|c|c|c|}
\hline Combination & $\operatorname{TFl}\left(\mathrm{mm}^{2}\right)$ & $\operatorname{TF} 2\left(\mathrm{~mm}^{2}\right)$ & $\operatorname{TF} 3\left(\mathrm{~mm}^{2}\right)$ & Combination & $\operatorname{TFl}\left(\mathrm{mm}^{2}\right)$ & $\operatorname{TF} 2\left(\mathrm{~mm}^{2}\right)$ & $\operatorname{TF3}\left(\mathrm{mm}^{2}\right)$ \\
\hline I & \multirow{16}{*}{$5 \times 2.5$} & \multirow{4}{*}{$5 \times 2.5$} & $5 \times 2.5$ & 33 & & \multirow{4}{*}{$5 \times 2.5$} & $5 \times 2.5$ \\
\hline 2 & & & $5 \times 3$ & 34 & & & $5 \times 3$ \\
\hline 3 & & & $5 \times 3.5$ & 35 & & & $5 \times 3.5$ \\
\hline 4 & & & $5 \times 4$ & 36 & & & $5 \times 4$ \\
\hline 5 & & \multirow{4}{*}{$5 \times 3$} & $5 \times 2.5$ & 37 & & \multirow{4}{*}{$5 \times 3$} & $5 \times 2.5$ \\
\hline 6 & & & $5 \times 3$ & 38 & & & $5 \times 3$ \\
\hline 7 & & & $5 \times 3.5$ & 39 & & & $5 \times 3.5$ \\
\hline 8 & & & $5 \times 4$ & $4^{\circ}$ & $5 \times 3.5$ & & $5 \times 4$ \\
\hline 9 & & \multirow{4}{*}{$5 \times 3.5$} & $5 \times 2.5$ & $4 \mathrm{I}$ & & \multirow{4}{*}{$5 \times 3.5$} & $5 \times 2.5$ \\
\hline IO & & & $5 \times 3$ & 42 & & & $5 \times 3$ \\
\hline II & & & $5 \times 3.5$ & 43 & & & $5 \times 3.5$ \\
\hline I2 & & & $5 \times 4$ & 44 & & & $5 \times 4$ \\
\hline I3 & & \multirow{4}{*}{$5 \times 4$} & $5 \times 2.5$ & 45 & & \multirow{4}{*}{$5 \times 4$} & $5 \times 2.5$ \\
\hline I 4 & & & $5 \times 3$ & 46 & & & $5 \times 3$ \\
\hline I5 & & & $5 \times 3.5$ & 47 & & & $5 \times 3.5$ \\
\hline 16 & & & $5 \times 4$ & 48 & & & $5 \times 4$ \\
\hline I7 & \multirow{16}{*}{$5 \times 3$} & \multirow{4}{*}{$5 \times 2.5$} & $5 \times 2.5$ & 49 & \multirow{16}{*}{$5 \times 4$} & \multirow{4}{*}{$5 \times 2.5$} & $5 \times 2.5$ \\
\hline I8 & & & $5 \times 3$ & 50 & & & $5 \times 3$ \\
\hline I9 & & & $5 \times 3.5$ & $5 \mathrm{I}$ & & & $5 \times 3.5$ \\
\hline 20 & & & $5 \times 4$ & 52 & & & $5 \times 4$ \\
\hline $2 \mathrm{I}$ & & \multirow{4}{*}{$5 \times 3$} & $5 \times 2.5$ & 53 & & \multirow{4}{*}{$5 \times 3$} & $5 \times 2.5$ \\
\hline 22 & & & $5 \times 3$ & 54 & & & $5 \times 3$ \\
\hline 23 & & & $5 \times 3.5$ & 55 & & & $5 \times 3.5$ \\
\hline 24 & & & $5 \times 4$ & 56 & & & $5 \times 4$ \\
\hline 25 & & \multirow{4}{*}{$5 \times 3.5$} & $5 \times 2.5$ & 57 & & \multirow{4}{*}{$5 \times 3.5$} & $5 \times 2.5$ \\
\hline 26 & & & $5 \times 3$ & $5^{8}$ & & & $5 \times 3$ \\
\hline 27 & & & $5 \times 3.5$ & 59 & & & $5 \times 3.5$ \\
\hline 28 & & & $5 \times 4$ & 60 & & & $5 \times 4$ \\
\hline 29 & & \multirow{4}{*}{$5 \times 4$} & $5 \times 2.5$ & 6I & & \multirow{4}{*}{$5 \times 4$} & $5 \times 2.5$ \\
\hline 30 & & & $5 \times 3$ & 62 & & & $5 \times 3$ \\
\hline $3 \mathrm{I}$ & & & $5 \times 3.5$ & 63 & & & $5 \times 3.5$ \\
\hline $3^{2}$ & & & $5 \times 4$ & 64 & & & $5 \times 4$ \\
\hline
\end{tabular}

Table 6.2. Field size for each TF corresponding to each combination number (gray columns), e.g, the field size for combination I is $(5 \times 2.5,5 \times 2.5,5 \times 2.5)$; for combination 2 is $(5 \times 2.5,5 \times 2.5,5 \times 3)$. Tr to T4 yielded 64 combinations of field sizes, whereas $\mathrm{T}_{5}$ to $\mathrm{T}_{7}$, only $\mathrm{i} 6$ combinations, since only two TF were used (table 6.I). 


\subsubsection{Clinical system output}

\subsubsection{System's delivery efficiency}

The EMS can decrease the beam nominal range to zero. However, the use of almost all the 18 range shifter plates decreases the number of protons that exit the EMS. To quantify the protons lost due to the use of these plates, simulations with a single-beam aimed at the isocentre were performed with the system's nominal energy $(230 \mathrm{MeV})$ and the energies chosen for treatments $\mathrm{T}_{\mathrm{I}-7}$. The AA was added to the simulations with the field sizes ranging from $5 \times 1 \mathrm{~mm}^{2}$ to $5 \times 5 \mathrm{~mm}^{2}$. The number of protons that arrived at the nozzle's exit window (figure 6.Ic) was scored and compared to the number of $\mathrm{IO}^{7}$ primary protons generated.

\subsubsection{Calculation of the dose rate and treatment time}

For practical reasons, it is important to assess whether the system would be able to deliver the prescribed dose of 2 Gy in an acceptable irradiation time $^{\left[r_{7}\right]}$, since the animal is under deep anesthesia and the common workflow includes imaging, treatment planning and irradiation. With a maximum beam current of Io $\mathrm{nA}$, the Mevion S250i HYPERSCAN, can produce 6.24 x ${ }_{\mathrm{IO}}{ }^{\text {Io }}$ protons per second. From the simulations described in section System's delivery efficiency, the time needed to deliver 2 Gy as function of energy and field size was estimated.

\subsection{Results}

\subsection{Proton plan that best covers the target}

Simulations of the seven treatments described in table 6.I were performed. All DVHs were scaled to the prescribed physical dose D9o $=2 \mathrm{~Gy}^{\mathrm{I}}$. Figure 6.3 (a) presents the D95 and D5 extracted from each of 64 dose distributions from Tr. The field size that produced the minimum difference between D95 and D5, i.e. the steepest slope of the tumour's DVH, is marked with a black line and hence chosen as the best combination for each treatment. Figure 6.3 (b) shows the VIo and V9o for the right lung and heart for the 64 field size combinations (explicitly in table 6.2), where the horizontal lines represent the V9o and Vio extracted from the photon plan. From the supplementary data 6.6, Figure 6.S.I, gives the results for $\mathrm{T}_{3}, \mathrm{~T}_{5}$ and $\mathrm{T}_{7}$.

\footnotetext{
${ }^{1}$ All doses reported in this work are physical doses (Gy) and not RBE-weighted absorbed doses (GyE).
} 

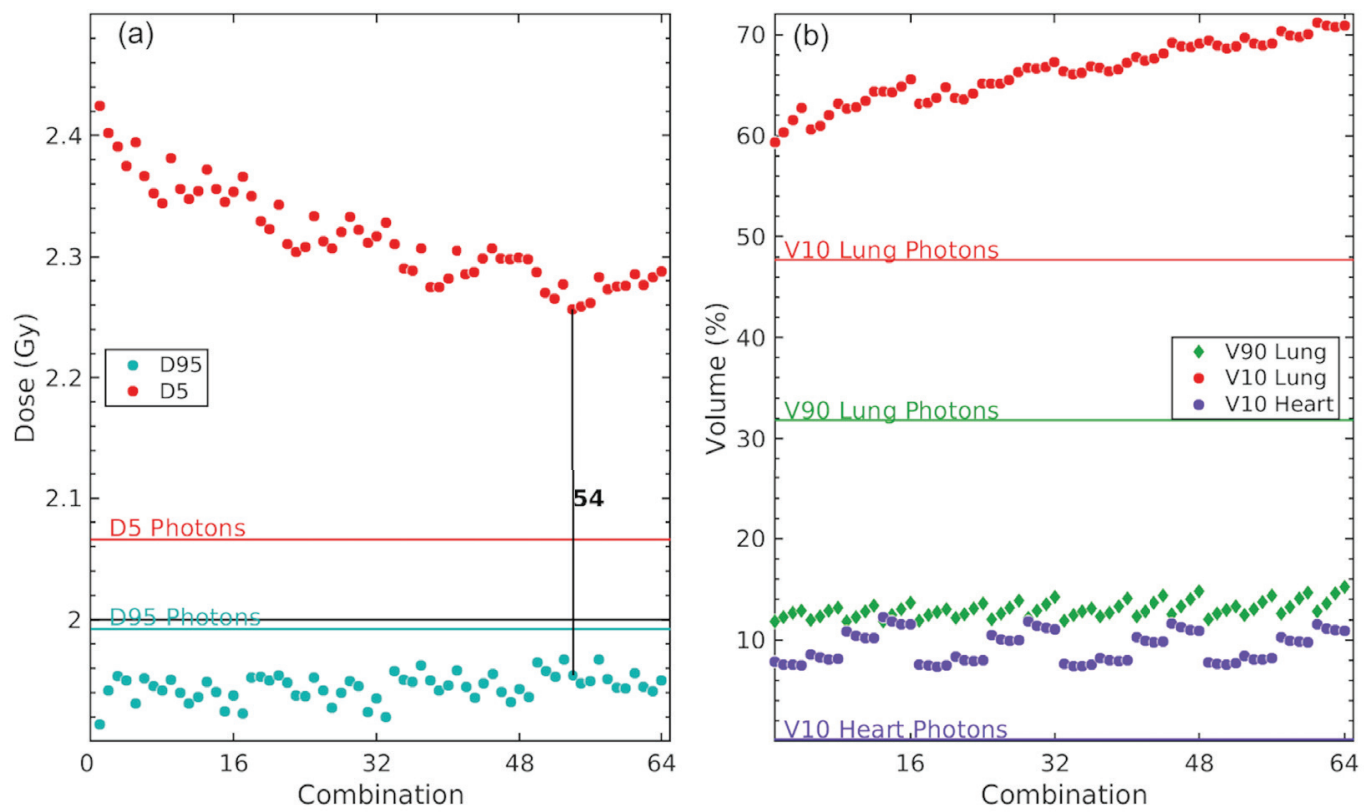

Figure 6.3. (a) D95 and D5 for all field combinations for $\mathrm{T}_{1}$, where the combination with the steepest slope between $\mathrm{D}_{95}$ and $\mathrm{D}_{5}$ is indicated with a black vertical line (combination ${ }_{54}$ ). The $\mathrm{x}$-axis shows the 64 combinations for $\mathrm{T}_{\mathrm{I}}$ : the first point on the $\mathrm{x}$-axis has the field size $\left(\mathrm{TF}_{\mathrm{I}}=5 \times 2.5 \mathrm{~mm}^{2}, \mathrm{TF}_{2}=5 \times 2.5\right.$ $\left.\mathrm{mm}^{2}, \mathrm{TF}_{3}=5 \times 2.5 \mathrm{~mm}^{2}\right)$ and the last $\left(\mathrm{TF}_{\mathrm{I}}=5 \mathrm{x}_{4} \mathrm{~mm}^{2}, \mathrm{TF}_{2}=5 \mathrm{x}_{4} \mathrm{~mm}^{2}, \mathrm{TF}_{3}=5 \mathrm{x}_{4} \mathrm{~mm}^{2}\right)$. All the 64 field-size combinations are shown in table 6.2. (b) representation of the ipsi-lateral right-lung VIo and the V9o, and the heart Vio. Horizontal lines show in both panels, as indicated, the D5, D95, Vio and V9o values extracted from the photon plan (figure 6.2).

The best case for each treatment scenario is described in table 6.3 in terms of the AA opening and the DVH metrics: D95, D5 for the tumour, Vio and Vgo for the ipsi-lateral right lung, and the Vio for the heart. Figure 6.4 shows the DVHs for the seven proton treatments, including the tumour, right lung and heart. The DVH extracted from the photon plan is shown in black. 


\begin{tabular}{|c|c|c|c|c|c|c|c|c|}
\hline \multirow{2}{*}{$\begin{array}{l}\text { Treatment } \\
\text { scenario \# }\end{array}$} & \multicolumn{3}{|c|}{ AA size for each TF } & \multirow{2}{*}{ D95 } & \multirow{2}{*}{ D5 } & \multirow{2}{*}{$\begin{array}{c}\text { V90 lung } \\
(\%)\end{array}$} & \multirow{2}{*}{$\begin{array}{c}\text { V10 lung } \\
(\%)\end{array}$} & \multirow{2}{*}{$\begin{array}{c}\text { V10 heart } \\
(\%)\end{array}$} \\
\hline & TF 1 & TF 2 & TF 3 & & & & & \\
\hline $\mathrm{TI}_{\mathrm{I}}$ & 4 & 3 & 3 & I. 95 & 2.26 & I3 & 69 & 8 \\
\hline $\mathrm{T}_{2}$ & 4 & 3 & 3 & I.95 & 2.27 & I3 & 69 & 8 \\
\hline $\mathrm{T}_{3}$ & 4 & 4 & 3 & I.96 & 2.18 & I2 & 72 & 2 \\
\hline $\mathrm{T}_{4}$ & 4 & 4 & 3 & 1.96 & $2.2 \mathrm{I}$ & I2 & 72 & I9 \\
\hline $\mathrm{T}_{5}$ & 4 & - & 3 & I. 98 & 2.2 & I9 & 7 & 5 \\
\hline $\mathrm{T} 6$ & 4 & - & 3.5 & 1.96 & 2.18 & 19 & 69 & 5 \\
\hline $\mathrm{T}_{7}$ & 4 & - & 3 & 1.96 & 2.27 & 23 & 8 & I7 \\
\hline Photons & - & - & - & I.99 & 2.07 & 32 & 48 & o \\
\hline
\end{tabular}

Table 6.3. Combinations that best covered the tumor found for each treatment scenario, indicating the adaptive aperture (AA) size for each treatment field (TF) and the dose volume histograms metrics $\mathrm{D}_{5}$, D95, Vio and V9o.

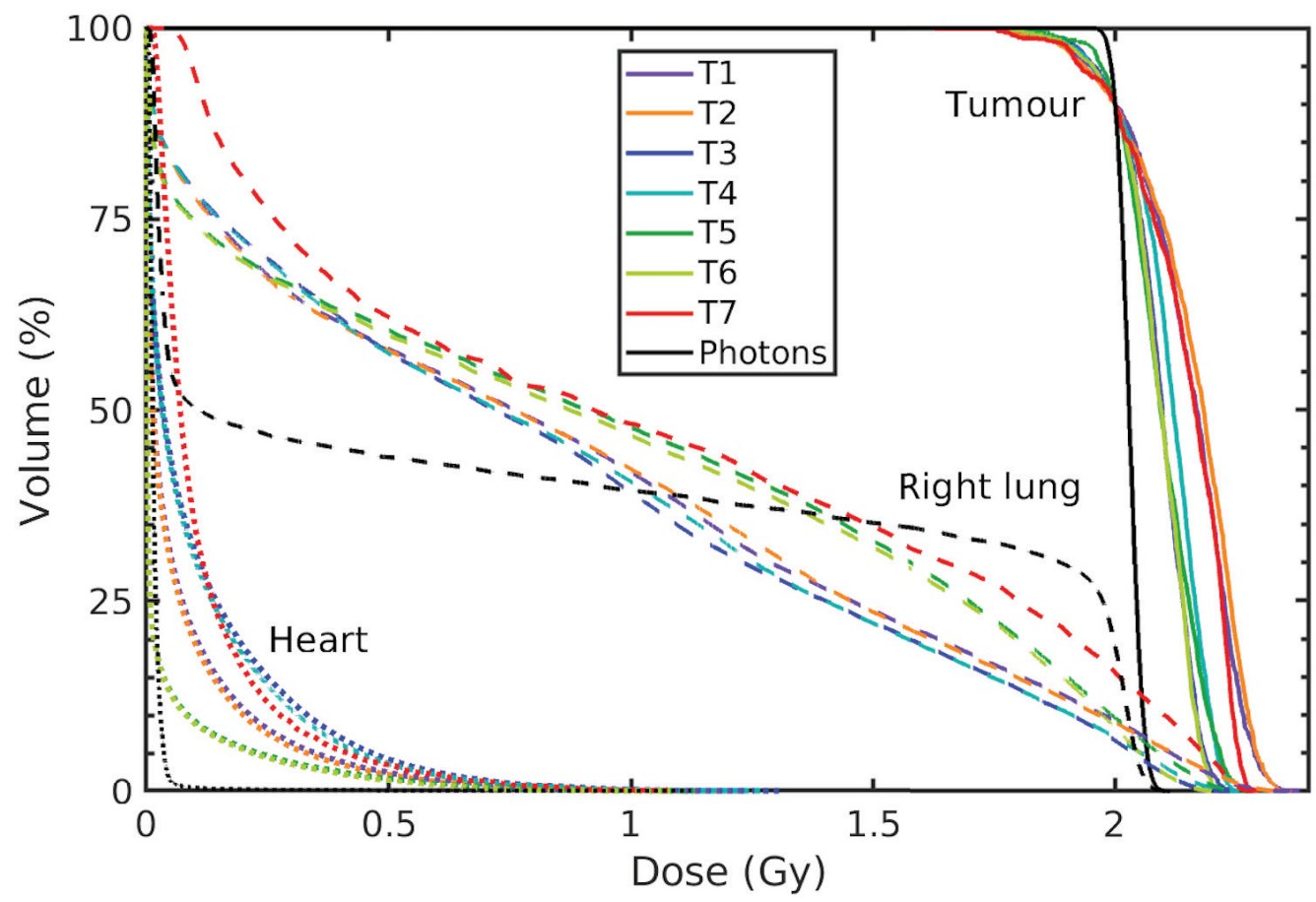

Figure 6.4. Dose volume histograms of the seven treatments for the tumour (solid line), ipsi-lateral right lung (dashed line) and heart (dotted line). 
Figure 6.5 (a-c) shows the dose distributions for three different types of proton irradiation: using three fields and low energies, using two opposing fields and low energies, and using two opposing "shoot-through" fields with high energies. Dose differences between these three irradiations are shown in figure $6.6(\mathrm{~d}-\mathrm{f})$.
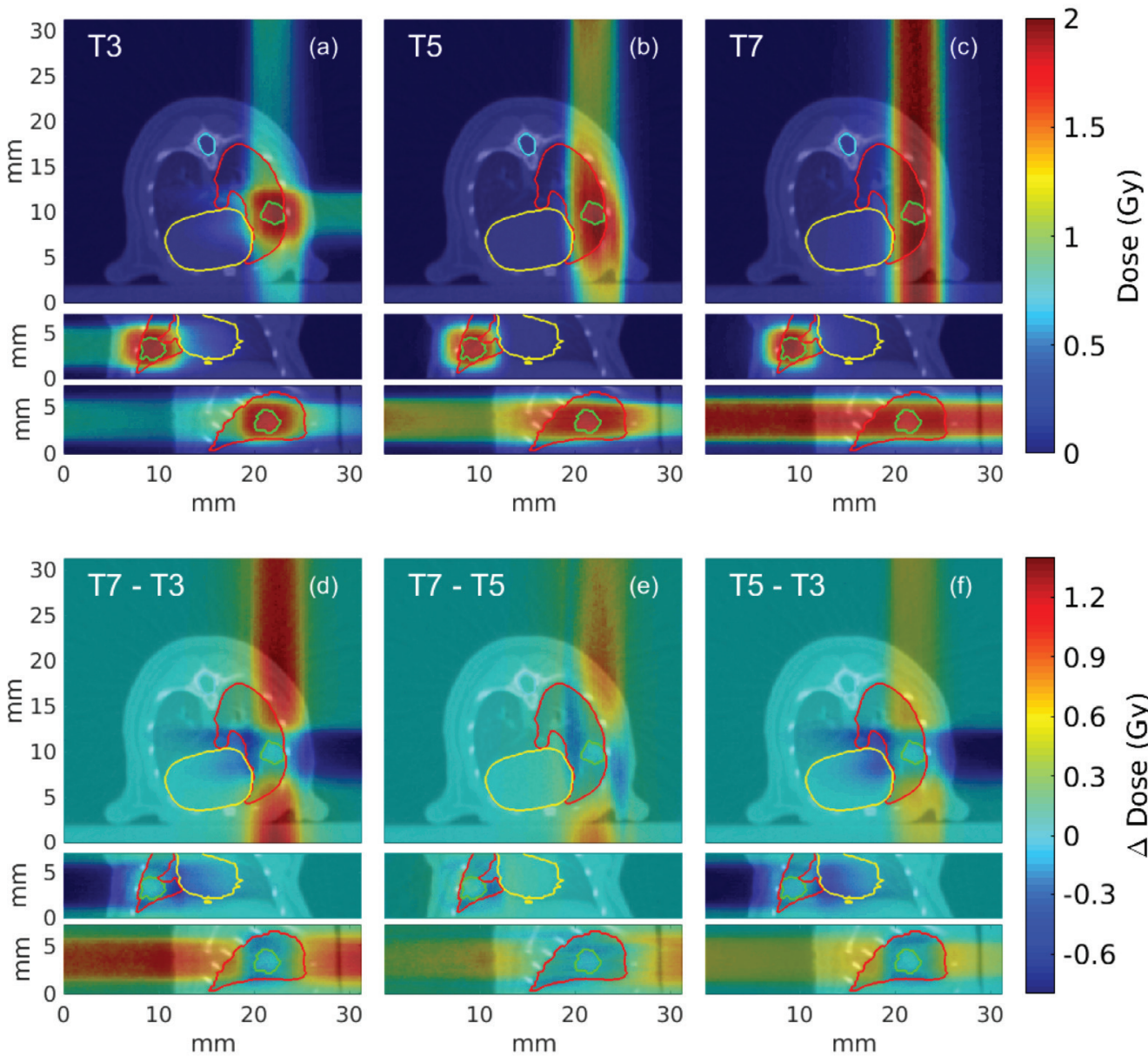

0.5

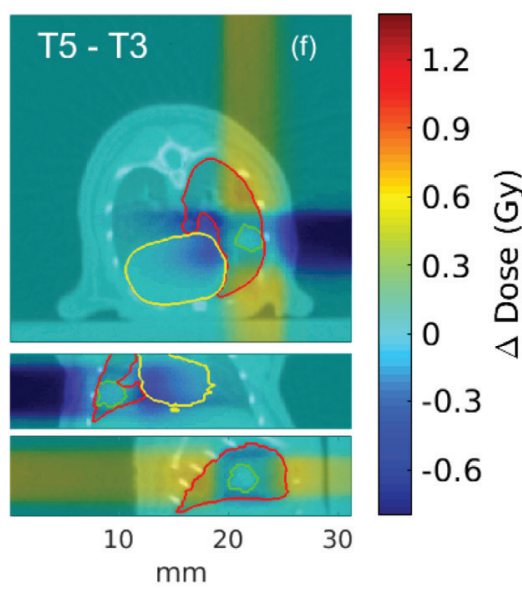

Figure 6.5. Proton dose distributions in transverse, coronal and sagittal planes for the field size combinations depicted in table 6.3 from irradiations $\mathrm{T}_{3}, \mathrm{~T}_{5}$ and $\mathrm{T}_{7}(\mathrm{a}, \mathrm{b}$ and c panels, respectively). Dose differences between irradiation $\mathrm{T}_{7}$ and $\mathrm{T}_{3}(\mathrm{~d})$, between $\mathrm{T}_{7}$ and $\mathrm{T}_{5}(\mathrm{e})$, and between $\mathrm{T}_{5}$ and $\mathrm{T}_{3}(\mathrm{f})$. 


\subsubsection{Clinical system output}

Figures 6.6(a-f) show the number of protons scored at the exit window of the nozzle for different field sizes, using a representative energy of $39.72 \mathrm{MeV}$. The fraction of protons for each energy that arrive to the exit window for an open field is shown in figure 6.7a, while figure $6.7 \mathrm{~b}$ gives these values as function of the field size.
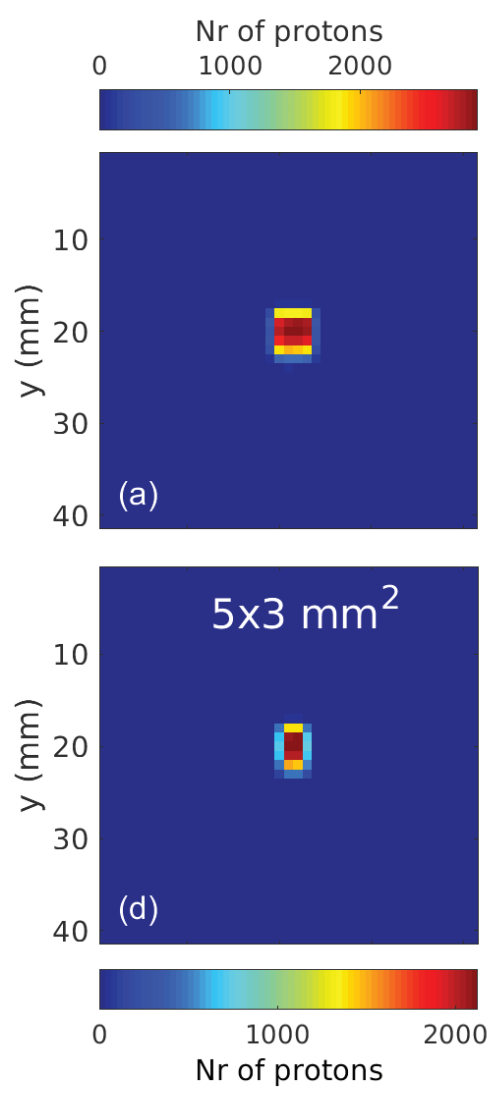
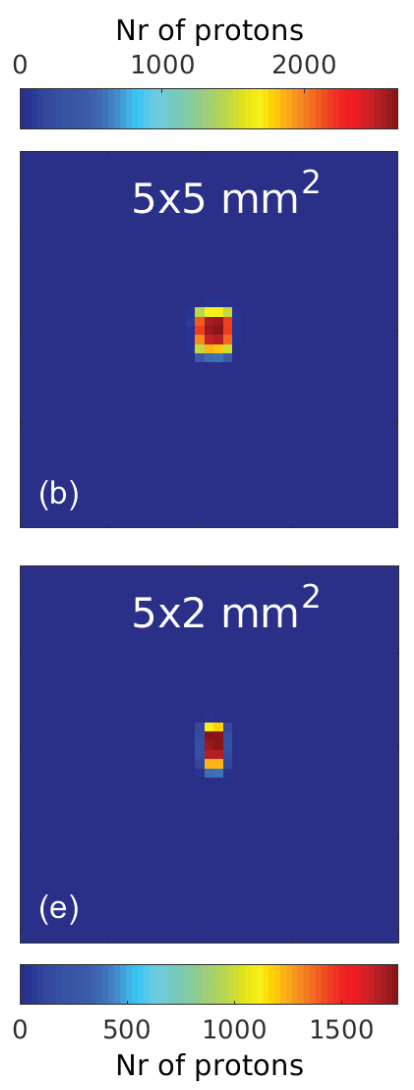
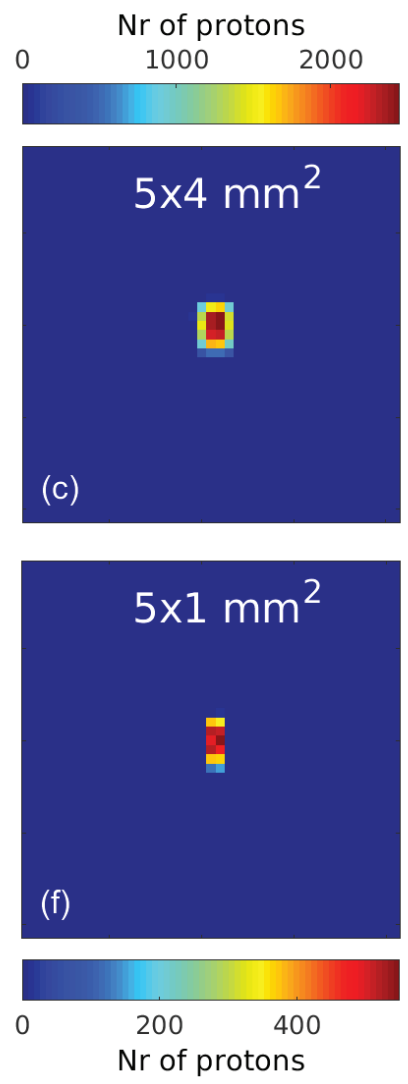

Figure 6.6. Number of protons scored at the exit window for a $39.72 \mathrm{MeV}$ beam, with different collimations (b-f) and with no collimation (a). 

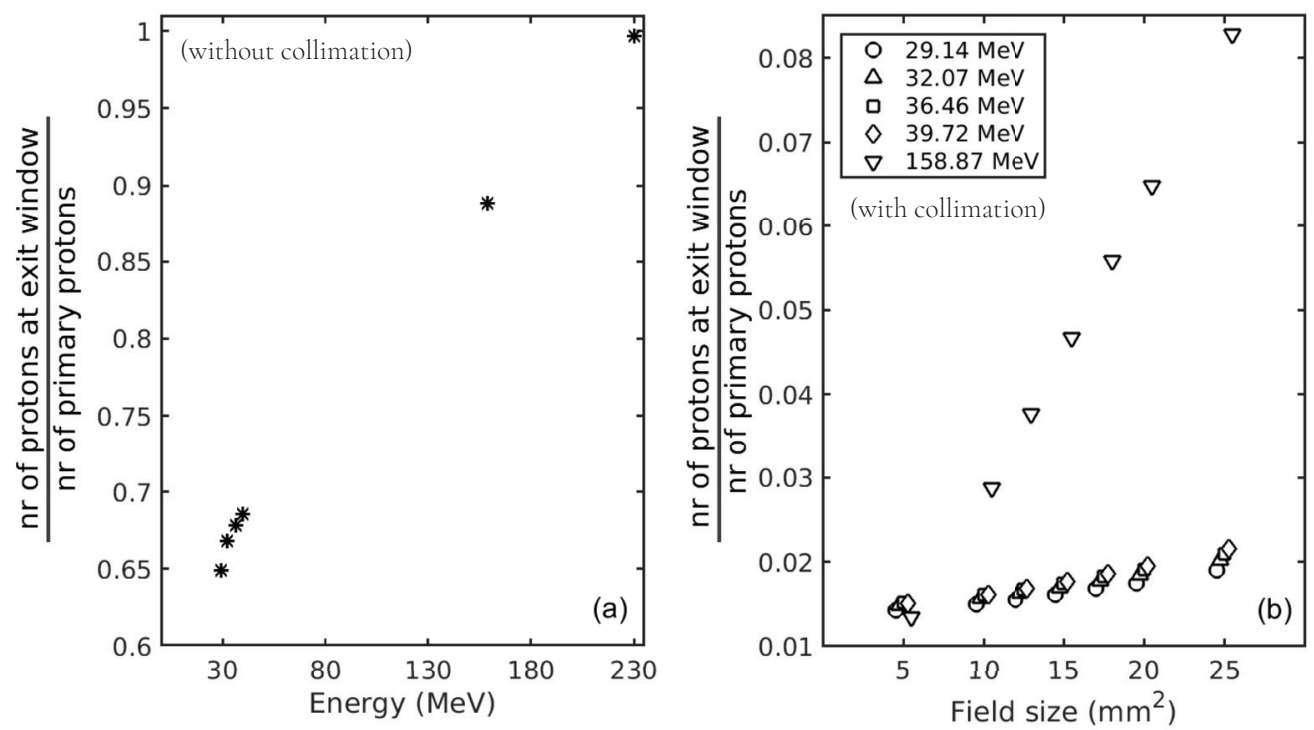

Figure 6.7. Fraction of protons that arrive at the exit window in an open field (no collimation) as function of the energy (a), and as function of the energy and field size (b). The field sizes used: $5 \mathrm{~mm}^{2}$, IO $\mathrm{mm}^{2}, \mathrm{I}_{2.5} \mathrm{~mm}^{2}, \mathrm{I}_{5} \mathrm{~mm}^{2}, 17.5 \mathrm{~mm}^{2}, 20 \mathrm{~mm}^{2}, 25 \mathrm{~mm}^{2}$, correspond to an adaptive aperture opening of $5 \mathrm{XI}$ $\mathrm{mm}^{2}, 5 \times 2 \mathrm{~mm}^{2}, 5 \times 2.5 \mathrm{~mm}^{2}, 5 \times 3 \mathrm{~mm}^{2}, 5 \times 3.5 \mathrm{~mm}^{2}, 5 \times 4 \mathrm{~mm}^{2}$ and $5 \times 5 \mathrm{~mm}^{2}$, respectively.

Considering the number of protons that the system produces per second at the exit of the cyclotron $\left(\sim 6.24 \mathrm{XIO}^{\mathrm{IO}}\right.$ protons for $\mathrm{IO} \mathrm{nA}$ beam current), the efficiency of each beam with respect to its energy and field size (figure $6.7 \mathrm{~b}$ ), the time to cover a 2 Gy target was determined and the results are shown in figure 6.8.

\subsection{Discussion}

This work presented a dosimetric study on the feasibility of pre-clinical irradiations with the compact proton system S250i with HYPERSCAN. Exploring the capabilities of the system EMS for range modulation, and AA for collimation, seven different proton irradiation scenarios were simulated in a MC platform to irradiate a mouse lung tumour. To perform the experiments simulated here, a high precision positioning platform with a coupled high-resolution CBCT imager would be necessary to assure a sub-millimetric uncertainty of the mouse positioning, while keeping the gantry at a fixed angle (figure 6.ra). This has already been achieved by 


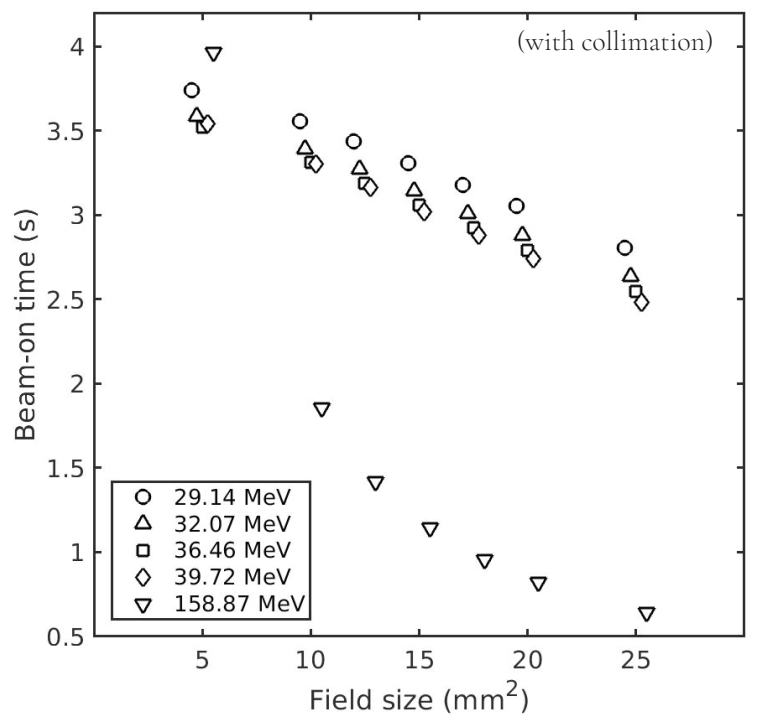

Figure 6.8. Beam-on time to deliver 2 Gy as function of the energy and field sizes.

coupling commercially available small animal x-ray imaging platforms to a fixed proton beam line $e^{[9]}$. On a gantry-based system, extra care has to be taken to position the beam nozzle in a reproducible fixed position.

The AA allows the creation of very small fields, limited to a minimum of $5 \mathrm{~mm}$ in the y-direction (axis in figure 6.Ib), but down to $0.5 \mathrm{~mm}$ in the $\mathrm{x}$-direction. Considering the size of the tumour and without explicitly adding any margin, a range of five AA openings were simulated, resulting in 16 or 64 different field size combinations. In the field of pre-clinical radiotherapy research, no guidelines are given for margins, which is an important study point recommended by the ESTRO ACROP report from Verhaegen et $\mathrm{al}^{[3]}$. In this respect, Vaniqui et $\mathrm{a}^{\left[{ }^{[8]}\right.}$ published a method of calculating the optimal collimator diameter from a set of fixed diameters depending on the parameters of a cost function, especially relevant for tumours affected by the breathing motion. Such an approach could be implemented in a follow-up study to this work, adapting the recipe proposed to the constrains of the AA of this system. 
Figure 6.3a plots the DVH metrics $\mathrm{D}_{5}$ and $\mathrm{D}_{95}$ for one scenario $\left(\mathrm{T}_{\mathrm{I}}\right)$, where the worst tumour coverage is seen for the smallest field $\left(5 \times 2.5 \mathrm{~mm}^{2}, 5 \times 2.5\right.$ $\mathrm{mm}^{2}, 5 \times 2.5 \mathrm{~mm}^{2}$ ) while generally improving for the largest fields. The bestcase tumour coverage, taken as the DVH with the steepest slope between $\mathrm{D}_{5}$ and $\mathrm{D}_{95}$, for $\mathrm{T}_{\mathrm{I}}$ was achieved for a field of $\left(5 \times 4 \mathrm{~mm}^{2}, 5 \times 3 \mathrm{~mm}^{2}, 5 \times 3\right.$ $\mathrm{mm}^{2}$ ). The same analysis for all seven treatments resulted in the seven best case scenarios that were shown in table 6.3 and in figure 6. 4. The "shootthrough" technique, with a similar beam arrangement as in the photon plan (figure 6.2(a)), would be expected to give a better coverage of the target. However, the use of a single spot beam resulted in a non-uniformity of the dose distribution in the lateral direction. Using an optimizer and smaller side-by-side beams may improve this result.

For benchmarking, a photon plan (figure 6.2a) was made by a commercially available treatment planning system for small-animal research. In contrast, in this work, single spot proton beams with equal weight were aimed at the isocentre. Nevertheless, figure 6.4 and table 6.3 show that the proton plans could be used for animal irradiation with a less optimal tumour coverage, but for most cases with an improved sparing of the OARs. Specifically, $32 \%$ of the right lung volume receives 90\% of the prescribed dose in the photon plan, which decreases to $\mathrm{I}_{2} \%$ in $\mathrm{T}_{3}$ and $\mathrm{T}_{4}$ proton plans. Furthermore, in figure $6.2 \mathrm{a}$ it is possible to observe a very high photon dose (three times the prescribed dose) in bone tissues, due to the predominance of the photoelectric effect for low energy $x$-ray beams. Protons in tissue also have a higher mass stopping power for bone, but only a factor 1.6 higher than water, in contrast with the factor of about 3 higher mass-energy absorption coefficient at low energy photon beams.

The proton treatments designed in this study used two strategies: low energy fields that stop in the tumour or high energy beams to shoot through. Dose differences show that the former allows for a decrease in dose to healthy tissues observed in the figure $6.5(\mathrm{~d}$, e and $\mathrm{f})$, with respect to the "shoot through" strategy. This would allow to study potential RBE effects ${ }^{[\mathrm{ro}]}$. The latter has the potential interest of performing small animal proton CT imaging that could be used for pre-treatment position verification or to improve range uncertainty by directly measuring the animal relative stopping power maps ${ }^{[19]}$. 
A special component of this beamline is the EMS, which is characterized by a constant range straggling at the Bragg peak for all energies. This might be a disadvantage for pre-clinical work, since it prevents the easy production of sharper beams. Another effect of the EMS is the increase in lateral beam spot size with the decrease in energy, caused by the many plates in front of the beam. For the low energies used in this work (Ti to T6), the beam spot size, taken as the standard deviation of a double Gaussian fit, is approximately $16 \mathrm{~mm}$. The AA can collimate the beam, however there is an intrinsic loss in efficiency by using large spot sizes with small fields, since most of the beam will be absorbed by the AA, clearly seen in figure 6.7 b. The central beam axis also suffers a decrease in intensity (figure 6.6(b-f)), since the very small angular deflections in proton trajectories are not negligible for small fields. Nevertheless, figure 6.8 shows the estimated irradiation time needed to deliver a 2 Gy dose with different energies and field sizes. For the lowest energy (29.I4 MeV) and the smallest field size (5xi $\left.\mathrm{mm}^{2}\right)$, the prescribed dose can be delivered in less than 5 seconds. The high dose rate of this system allows for 30 Gy to be delivered in less than a minute. 


\subsection{Conclusion}

The feasibility of the Mevion S250i with HYPERSCAN proton irradiation system for pre-clinical research was affirmatively tested, using different strategies in the choice of energies, field directions and sizes. Moreover, the system would be able to give very high doses in a short period of time, due to the system's high beam current. With the use of a movable platform equipped with $\mu$ CBCT imaging and a table with accurate positioning, the clinical proton system could be used to perform proton irradiation in small animals for a wide range of studies. 


\subsection{Supplementary Data}
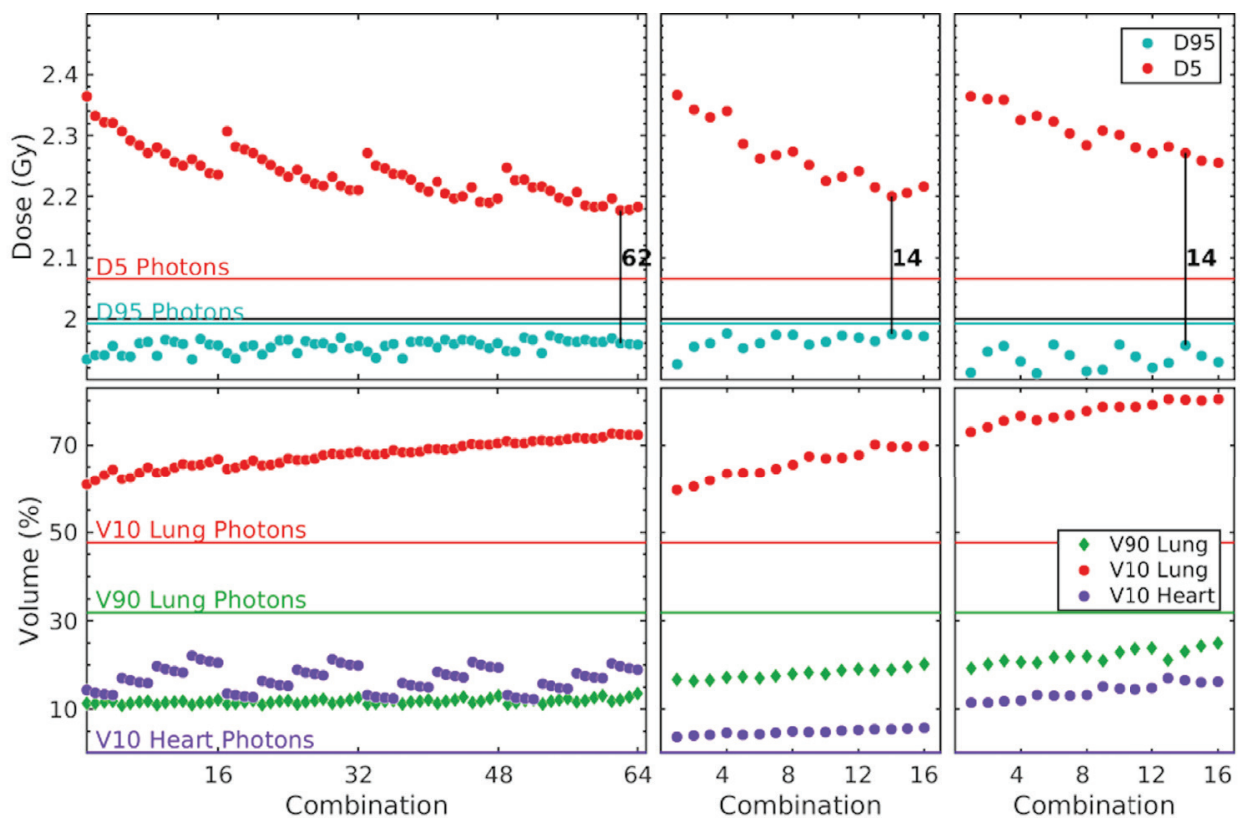

Figure 6.S.r. Top panels: $\mathrm{D}_{95}$ and $\mathrm{D}_{5}$ for all field combinations for $\mathrm{T}_{3}$, $\mathrm{T}_{5}$ and $\mathrm{T}_{7}$ (left, middle, right, respectively), where the combination with the steepest slope between D95 and D5 is indicated with a black vertical line. Bottom panels: representation of the ipsi-lateral right-lung Vio and the V9o, and the heart $\mathrm{V}_{10}$ for $\mathrm{T}_{3}, \mathrm{~T}_{5}$ and $\mathrm{T}_{7}$. Horizontal lines show in both panels, as indicated, the $\mathrm{D}_{5}$, D95, Vio and V9o values extracted from the photon plan (Figure 6.2). 


\subsection{References}

${ }^{[1]}$ B.F. Koontz, F. Verhaegen, D. De Ruysscher, Tumour and normal tissue radiobiology in mouse models: how close are mice to mini-humans?, Br J Radiol, 90 (2017) 20r6044I.

${ }^{[2]}$ F. Verhaegen, P. Granton, E. Tryggestad, Small animal radiotherapy research platforms, Phys Med Biol, 56 (20II) R55-83.

${ }_{33}$ F. Verhaegen, L. Dubois, S. Gianolini, M.A. Hill, C.P. Karger, K. Lauber, K.M. Prise, D. Sarrut, D. Thorwarth, C. Vanhove, B. Vojnovic, R. Weersink, J.J. Wilkens, D. Georg, ESTRO ACROP: Technology for precision small animal radiotherapy research: Optimal use and challenges, Radiother Oncol, I26 (2018) 47I-478.

${ }_{4]}^{[4}$ F. Verhaegen, S. van Hoof, P.V. Granton, D. Trani, A review of treatment planning for precision image-guided photon beam pre-clinical animal radiation studies, Z Med Phys, 24 (2014) 323-334.

${ }^{55}$ J. Wong, E. Armour, P. Kazanzides, I. Iordachita, E. Tryggestad, H. Deng, M. Matinfar, C. Kennedy, Z. Liu, T. Chan, O. Gray, F. Verhaegen, T. McNutt, E. Ford, T.L. DeWeese, Highresolution, small animal radiation research platform with $\mathrm{x}$-ray tomographic guidance capabilities, Int J Radiat Oncol Biol Phys, 7I (2008) I59I-I599.

${ }^{[6]}$ S.J. van Hoof, P.V. Granton, F. Verhaegen, Development and validation of a treatment planning system for small animal radiotherapy: SmART-Plan, Radiother Oncol, Iog (2013) 36I-366.

${ }^{[7]}$ C. Greubel, W. Assmann, C. Burgdorf, G. Dollinger, G. Du, V. Hable, A. Hapfelmeier, R. Hertenberger, P. Kneschaurek, D. Michalski, M. Molls, S. Reinhardt, B. Roper, S. Schell, T.E. Schmid, C. Siebenwirth, T. Wenzl, O. Zlobinskaya, J.J. Wilkens, Scanning irradiation device for mice in vivo with pulsed and continuous proton beams, Radiat Environ Biophys, 50 (2OII) 339-344.

[8] J. Constanzo, M. Fallavier, G. Alphonse, C. Bernard, P. Battiston-Montagne, C. Rodriguez-Lafrasse, D. Dauvergne, M. Beuve, Radiograaff, a proton irradiation facility for radiobiological studies at a $4 \mathrm{MV}$ Van de Graaff accelerator, Nuclear Instruments and Methods in Physics Research Section B: Beam Interactions with Materials and Atoms, 334 (2014) 52-58.

${ }^{[9]}$ E. Ford, R. Emery, D. Huff, M. Narayanan, J. Schwartz, N. Cao, J. Meyer, R. Rengan, J. Zeng, G. Sandison, G. Laramore, N. Mayr, An image-guided precision proton radiation platform for preclinical in vivo research, Phys Med Biol, 62 (2017) 43-58.

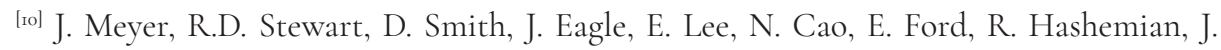
Schuemann, J. Saini, S. Marsh, R. Emery, E. Dorman, J. Schwartz, G. Sandison, Biological and dosimetric characterisation of spatially fractionated proton minibeams, Phys Med Biol, 62 (2017) 9260-928I. 
${ }^{[n]}$ R.P. Coppes, C.T. Muijs, H. Faber, S. Gross, J.M. Schippers, S. Brandenburg, J.A. Langendijk, P. van Luijk, Volume-Dependent Expression of In-Field and Out-of-Field Effects in the Proton-Irradiated Rat Lung, International Journal of Radiation Oncology • Biology - Physics, 8I (2011) 262-269.

$\left.{ }^{\left[{ }^{2}\right]}\right]$ J. Müller, E. Beyreuther, T. Suckert, C. Neubert, L. Karsch, C. von Neubeck, J. Pawelke, M. Schürer, M. Krause, A. Lühr, Establishment of small animal irradiation at University Proton Therapy Dresden. Paper presented at the Fourth International Conference on Precision Image-Guided Small Animal Radiotherapy Research, Lisbon, 2018.

${ }^{\left[{ }_{3}\right]}$ E. Diffenderfer, M.M. Kim, P. Irmen, K. Shoniyozov, S. Tuttle, C. Koumenis, L. Dong, J. Metz, Design and commissioning of an image-guided small animal radiation platform for proton radiobiology research. Paper presented at the Fourth International Conference on Precision Image-Guided Small Animal Radiotherapy Research, I2-I4 March 2018, , Lisbon.

${ }^{\left[{ }_{4}\right]}$ D.J. Brenner, C.D. Elliston, E.J. Hall, H. Paganetti, Reduction of the secondary neutron dose in passively scattered proton radiotherapy, using an optimized pre-collimator/collimator, Physics in medicine and biology, 54 (2009) 6065-6078.

${ }^{\left[{ }_{5}\right]}$ J. Perl, J. Shin, J. Schümann, B. Faddegon, H. Paganetti, TOPAS: An innovative proton Monte Carlo platform for research and clinical applications, Medical Physics, 39 (2012) 68г $8-6837$.

${ }^{[16]}$ C.J. Zacharatou, H. Paganetti, Physics Settings for Using the Geant4 Toolkit in Proton Therapy, IEEE Transactions on Nuclear Science, 55 (2008) Ior8-IO25.

${ }^{\left[{ }_{7}\right]}$ M. Balvert, S.J. van Hoof, P.V. Granton, D. Trani, D. den Hertog, A.L. Hoffmann, F. Verhaegen, A framework for inverse planning of beam-on times for $3 \mathrm{D}$ small animal radiotherapy using interactive multi-objective optimisation, Phys Med Biol, 6o (2015) 568I5698.

${ }^{\left[{ }^{18}\right]}$ A. Vaniqui, B. van der Heyden, I.P. Almeida, L.E.J.R. Schyns, S.J. van Hoof, F. Verhaegen, On the determination of planning target margins due to motion for mice lung tumours using a four-dimensional MOBY phantom, The British Journal of Radiology, $9^{2}$ (2019) 20180445 .

${ }^{\left[{ }^{2}\right]}$ R.W. Schulte, S.N. Penfold, Proton CT for Improved Stopping Power Determination in Proton Therapy, invited, Transactions of the American Nuclear Society, Io6 (2012) 55-58. 

Summary, discussion, and prospective work 


\section{I Summary and discussion}

The rise of small-animal image-guided precision radiotherapy platforms has been revolutionizing translational and radiobiological research. Modern devices and current advanced tumour models have enabled fundamental research on irradiation protocols, fractionation regimens, adjuvant therapies and established concepts in controlled environments, with large (transgenic) animal cohorts and accelerated results. A number of biological, physical and technological concerns remain. A recent report from the European society for radiotherapy and oncology advisory committee in radiation oncology practice (ESTRO ACROP) numbered the main challenges to be addressed in this field ${ }^{[r]}$; it enquires about:

- the key technologies for downscaling clinical treatments and the imaging modalities to be integrated and potentially used in treatment planning;

- the treatment of target motion and optimal irradiation margins;

- the accuracy and precision of small field dosimetry and dose distribution verification;

- the difference between high and low-energy photon irradiation.

Although categorized into different topics, the challenges have a synergetic relationship: imaging requires sub-millimetric resolution, affects dosimetry and is affected by motion; and pre-clinically mimicking clinical scenarios requires lower energies to avoid large beam penumbras and dose (re-)buildup regions which also affect dosimetry and might yield different treatment outcomes if dose calculations do not consider medium heterogeneities.

This thesis has briefly introduced the historical evolution of radiotherapy and the need for pre-clinical EBRT research (Chapter I). It presented ways to tackle some of these challenges and warned about their drawbacks. In the following sections, the research concerning these and additional points is summarized, discussed and future perspectives from discussion points are canvassed. 


\section{I.I Imaging and dose calculations}

In a framework to establish imaging protocols that allow accurate dose calculations, Chapter 2 examined the feasibility of using dual energy CT (DECT) to extract tissue atomic compositions and assess dose calculation accuracy gain. The study demonstrated improved accuracy for DECT in comparison to SECT. It stressed the arbitrariness of the broadly used SECT method: it is not clear which media and how many linear segments should be used for generating a calibration curve, and where to place material boundaries. The latter are often selected based on visual inspection, yielding inter-individual differences. In this study, three main debatable topics were analysed: tissue compositions, energy dependence and image quality.

Segmenting a specimen using only a single dense bone material with elevated effective atomic number caused high absorption, resulted in less photoelectric interactions in soft tissue, higher dose deposition in osseous tissues and underestimation in the organs at risk (OARs) in proximity of bone. On the other hand, the choice of multiple bone types, using DECT, resulted in lower dose values for the different tissues occupying the same volume and minimal assignment of (human) cortical bone (I.9\%). It was recommended to segment mice using bony tissues with decreased density and effective atomic number values considering that their bones are flexible and possibly closer to human cartilage in composition. Additionally, incorrect material segmentation was shown to be aggravated with a decrease in beam energy due to the intensified role of photoelectric cross-sections, causing materials with different effective atomic numbers to absorb increasingly different fractions of energy. These two subjects, energy dependence and tissue compositions, are echoed throughout this thesis and have a deterministic relationship in this field.

Although necessary for MC dose calculations, tissue compositions have a marginal impact on clinical EBRT photon treatments, where incoherent interactions are predominant and dependent on tissue electron density ${ }^{[2]}$. Their influence is higher on clinical proton EBRT and $\mathrm{kV}$ techniques such as brachytherapy and small animal radiotherapy. For clinical protons beams, they influence the mean excitation energy used to calculate the proton stopping power ratio and affect range verification techniques. For $\mathrm{kV}$ techniques, although Compton interactions are still important, photoelectric probability is significant and this effect is strongly dependent on the atomic number of the medium $\left(\mathrm{Z}^{3^{\sim}}\right)$. The mass-energy absorption 
coefficients, a quantity closely related to absorbed dose, may differ by more than a factor of six for low energies ${ }^{[3]}$.

The assignment of tissue compositions is a bottleneck in radiotherapy. In pre-clinical EBRT, human tissue compositions are predominantly used due to the scarce literature on animal ones. A small number of studies has presented quantitative data on animal tissues ${ }^{[4-9]}$, albeit unspecific for radiotherapy or focused on trace-elements. The increased biological interest on molecular or cellular entities rather than atomic ones and the practical difficulties in establishing atomic compositions are possible culprits. Clinical radiotherapy uses human tissue reference data listed in reports 46 of the International Commission on Radiation Units and Measurements (ICRU) and report 23 from the International Commission on Radiological Protection (ICRP) ${ }^{[10-12]}$.

Mann-Krzisnik has published a thorough study, which describes how the human reference data was established ${ }^{\left[{ }^{3}\right]}$. It reports that measurements were taken between 1940 and 1960. Lighter elemental composition (hydrogen, carbon, nitrogen and oxygen) was extrapolated from gross proportions of water, fat, proteins and carbohydrates while heavier elements were derived from spectrographic analysis of ashed and dried tissue samples extracted from cadavers. A number of issues was observed, e.g. some tissue compositions did not sum up to Ioo\% or did not contain carbon, which is considered impossible for a biological tissue. Data was later rendered by several sources and renormalized, which may have added inconsistencies as individual studies started from different premises (e.g. distinct levels of hydration or manipulation methods). Additionally, lighter elemental composition derivations may have neglected variations in chemical composition of fats, proteins and carbohydrates and the method employed for heavier elements possibly lacked precision. All measurements were derived from ex vivo samples, which might differ from in vivo ones in terms of tissue compositions, hydration and densities. Moreover, akin to elemental composition, ICRU and ICRP density reference data is connected to the same studies, rendered from several publications and prone to similar issues. Different from elemental compositions, density is typically derived from CT scans rather than reference data, hence the importance of this reference parameter is decreased.

Dosimetric implications of using human tissue compositions in mice have been investigated with DECT and differences between human and 
murine elemental compositions led to dose errors of up to $20.1 \%$ for bones in addition to a large spread in mass-energy absorption coefficients for different tissues ${ }^{[14]}$. These results stress the need of research on reference data or, minimally, establishing a relationship between human and animal tissues; for murine studies (and for other specimens used for radiotherapy research), population samples should include different strains, genders and ages as concentration of certain elements vary within these categories. Moreover, the large spreads indicate that assigning one mean reference value to a whole organ is not realistic as there is a distribution of compositions. New technologies should be explored to derive updated quantities both in invasive and non-invasive ways. This topic is revisited in section 7.2.I.

Finally, Chapter r raised an image quality discussion motivated by extensive artefacts and noise on the CT scans. For the former, partial volume effects can explain the high source of errors on the boundary regions. For the latter, rather than uniform, the bulk of the phantom displayed a texturelike appearance, with irregular CT numbers $(42 \pm 62$ and $16 \pm 57$ HU for 50 and $90 \mathrm{kVp}$ scans) possibly due to noise; moreover the DECT material image, effective atomic number $\left(Z_{\text {eff }}\right)$ image, was noisy and presented a mean $Z_{\text {eff }}$-value encompassing several soft tissues (mean of 8.0 \pm o.4, ranging from 6.0 to I0.7). As the CT projections were reconstructed with a simple Feldkamp-Davis-Kress (FDK) back-projection algorithm, it was presumed that the usage of iterative reconstruction algorithms with artefact correction kernels could improve the effect of noise and provide superior DECT material segmentation.

\section{I.2 Iterative methods for image reconstruction}

To analyse the effect of different image reconstruction techniques on image quality and DECT metrics, Chapter 3 presented a software platform for pre-clinical CBCT image reconstruction. Pre-processed projections were reconstructed using the analytical filtered back-projection (FDK) or the iterative reconstruction (IR) algorithms, e.g. iterative FDK, simultaneous algebraic reconstruction technique (SART), simultaneous iterative reconstruction technique (SIRT) or Conjugate Gradient (CG). A cupping correction was included, a polynomial pre-correction to the attenuation data for linearization. Imaging metrics were quantitatively assessed with a quality assurance phantom and DECT analysis was performed to determine the influence of each reconstruction technique on the relative electron density $\left(\rho_{\mathrm{e}}\right)$ and $Z_{\text {eff }}$ values. 
IR methods proved computationally costly due to the large matrices and small pixel sizes chosen for the study but presented satisfactory results with smaller grids, typical of pre-clinical practice. Nevertheless, reconstruction times were longer when compared to analytical methods as these algorithms use multiple repetitions. With increased computational power and the use of graphical processing units (GPUs), both with current technology, this difference can become negligible and not represent a limiting factor for online iterative reconstruction: time is of the essence in this field as animals are imaged while restrained under sedation and long anaesthesia times might impair the fitness of the specimen and the integrity of the study; this topic is further discussed in section 7.2.2.

The cupping artefact correction provided accurate CT numbers and could benefit larger subjects and applications that require increased image accuracy. The workflow needs improvement, as reconstructing an object a number of times to derive a polynomial, which will correct a final reconstruction, is a lengthy and impractical process. Either a fixed polynomial order could be chosen in advance according to the object size or different algorithms could be applied. Regarding DECT, IR showed significantly smaller spread for each material in the $\rho_{\mathrm{e}}-Z_{\text {eff }}$ space and lower $Z_{\text {eff }}$ and $\rho_{\mathrm{e}}$ residuals (on average $24 \%$ and $25 \%$ lower, respectively). This is an important result as it indicates that imaging noise was reduced and the IR methods provided tissue composition distributions closer to the reference values. Thus, IR could benefit DECT tissue segmentation as the spread in $\rho_{\mathrm{e}}-Z_{\text {eff }}$ space could be closer to the spread in tissue values, and less affected by errors intrinsic to the imaging modality. A myriad of iterative methods exists and provide different degrees of accuracy and computational times. This study showed the development of the software and a first application to small animal CBCT. Other algorithms, and back-projection, forwardprojection and window type filters, already foreseen in the software, should be further characterized and additional artefact kernels further implemented to expand the reach of these techniques.

Considering the image quality aspect, the analysed reconstruction techniques provided acceptable and similar geometric accuracy, uniformity and CT number results. SART provided improved contrast-to-noise ratio (CNR) but lower resolution for some media, when compared to the other techniques. Accuracy on image metrics is crucial for dose calculations, as the contours of anatomical structures can suffer from reduced resolution, abundance of noise or non-uniformity. Geometric accuracy and contrast 
are also important for spatial targeting and measurements of anatomical structures, which can determine how a tumour or an OAR is responding to treatment. The influence of the scanning parameters in terms of current, frame rate, rotational speed, gain and exposure were adopted from established protocols and could be revisited. It would be beneficial to investigate if lower exposure times associated with IR methods would present similar CNR and noise levels at lower imaging doses. High imaging doses from cumulative scans may change the animal immune response and other biological pathways that may alter the experimental outcome $e^{[5-17]}$. Thus, quantification of imaging dose is an important part of system commissioning and operation, especially for longitudinal studies. The use of imaging techniques that provide no radiation dose is also advised, when possible.

Furthermore, an image quality assurance (QA) program should be in effect, following guidelines, which should provide tolerances for normal operation tests, and take corrective actions such as imaging system recalibration if tolerance levels are not met when recommended imaging protocols are used. The QA tests should happen periodically. Currently, due to the lack of specific recommendations, several research institutions have different QA approaches, ranging from extensive tests to the absence of this practice. The $4^{\text {th }}$ Conference on Small Animal Precision Image-Guided Radiotherapy, Lisbon, March 2018, held discussions concerning this topic. Several experts agreed on the need for recommendations and that meanwhile users should design ways to ensure the calibration of their systems. It is possible to find publications with acceptable QA tests ${ }^{[18,19]}$. Beyond imaging, the QA program should also include the dosimetry of system, on an annual basis, to estimate the dose rate of the $\mathrm{x}$-ray tube, using an appropriate ionization chamber and correction factors. Following the American Association of Physicists in Medicine (AAPM) Radiation Therapy Committee Task Group ${ } \mathrm{I}^{[20]}$, or similar recommendations ${ }^{[2-23]}$, for reference dosimetry of low- and medium energy x rays for radiotherapy and radiobiology.

\section{I.3 Irradiation margins}

As mentioned on section 7.I, the treatment of target motion and optimal irradiation margins constitutes a challenge for small animal EBRT. In an attempt to confront these issues, the research presented in Chapter 4 analysed the effect of respiratory motion on irradiation margins for murine lung tumours. Four-dimensional $\left({ }_{4} \mathrm{D}\right)$ mathematical phantoms with 
different lung tumour locations and extreme breathing curves were used for dose calculations of a $360^{\circ}$ are treatment plan. A time-resolved dose was derived, considering the irradiator gantry rotation and the breathing motion. From different radiotherapy metrics, it was understood that the effect of respiratory motion is dependent on the breathing pattern and the tumour position. Ultimately, a recipe, which considers the irradiation collimator size, was proposed to derive tumour margins and spare OARs by respecting constraints on user-defined metrics. Moreover, the addition of a target margin, especially on sites where movement is substantial, was recommended.

Extreme breathing scenarios were used to represent the possible range where the actual breathing pattern would fall. It varies according to the anaesthesia protocol and the respiratory and cardiac rates. A sedation scenario with minimal motion while physically restraining the specimen is ideal. This study generated a few recommendations to avoid sub-optimal pulmonary treatments. Regarding the orthotopic tumour implantation, when possible, it is recommended to avoid the diaphragm region, as it is the region most affected by motion. Implantations on the superior lobe of the right lung are advised, as the left lung is closer to the heart, an important OAR. The use of motion tracking and gating techniques for imaging and irradiation allow physiological heart and lung motion to have a decreased impact on imaging artefacts and dose heterogeneities ${ }^{[24,25]}$.

Different gating approaches have been documented and rely on the type of scanner, the specimen and the research in question. In prospective gating, data acquisition is triggered by a motion signal. Prospective methods are used in scanners that operate under step-and-shoot conditions. The x-ray tube is triggered by a user-supplied signal based on the animal physiological motion. Respiratory and cardiac trigger signals can be generated, e.g., with a mechanical ventilator at a selected point in the respiratory cycle, from real-time measurements of a pneumatic cushion positioned on the diaphragm, optical measurements of diaphragm motion, measurements of the heart rate or electrocardiogram (ECG) signal ${ }^{[26-33]}$. Retrospective gating is performed continuously and all motion correction is applied during post-processing. It can be performed both under continuous rotation or in step-and-shoot mode. Projection images and cardiac and respiratory signals are simultaneously and uninterruptedly recorded. Post-processing includes projection sorting based on the recorded physiological phase of the animal at the time each projection was acquired. Projections acquired in the same 
phase are reconstructed ${ }^{[34-36]}$. Image-based gating determines the portion of the breathing or cardiac cycle in the projection space ${ }^{[37-39]}$. Different $x$-ray shutter subsystems have been developed for both commercially available small animal irradiators ${ }^{[40-42]}$, although only one subsystem is in commercial stage. They allow beam gating during treatment, with irradiation only taking place during the breathing cycle when tissue movement is minimal. They facilitate dose delivery during animal movement and are particularly well suited for irradiation of targets within the chest and abdomen. Although not used for the research of Chapter 3, gating techniques contribute to the treatment conformality and are likely to be implemented in most precision irradiators in the near future.

Finally, the use of margins is recommended. This study used a cost function based on dose-volume histogram (DVH) parameters and the available collimators. However, the recipe can be adapted according to adjustable constraints or collimation devices and is independent of the breathing curve; it could be applied for other respiratory or irradiation regimens. A thorough recipe for establishing the concept of planning target volume (PTV) should consider additional parameters such as setup and fractionation errors, when applicable. An important conclusion from this study is the need of considering anatomical and physiological characteristics during clinical to pre-clinical translation, or vice-versa. Although margins are sometimes extrapolated from clinical experience, they might not be representative for certain small animals.

Independently of the margin function, the investigation of an interpolation function for dose accumulation from different anatomic phases to a reference phase using a rigid grid (direct dose mapping) was proposed for speeding up the dose calculation process, as a first approximation: tissue deformation and the cardiac motion were not modelled. Calculation times at least ro times faster are promising for further development of the technique. However, results may depend on the targeted site and surrounding anatomy. Moreover, as the first time point was considered the reference phase, the dose map derived from the CBCT scan could be subject to noise and blurring. This point was investigated through simulation of the full breathing cycle and effect of respiratory motion on the CT scan blurring was considered minimal while tumour shape was highly preserved. The presented technique could potentially be used for adaptive radiotherapy, which may require the daily delivered dose to be accumulated. 


\section{I.4 Dose reporting}

Another topic highlighted by the ESTRO ACROP report, mentioned in section 7.I, is the treatment (or lack thereof) of different dose reporting quantities generated by the various dose calculation algorithms. Conventional and rather simpler dose calculations methods, which considered the human body as water-equivalent and reported dose to water, have evolved towards sophisticated model-based algorithms e.g. MC. The latter transports radiation through heterogeneous media and report the absorbed dose to a reference medium embedded in a surrounding transport medium. Different dose reporting quantities have been derived using the cavity theory: they are dose-to-medium-in-medium $\left(D_{\mathrm{mm}}\right)$, dose-to-water-inmedium $\left(D_{\mathrm{wm}}\right)$ or dose-to-water-in-water $\left(D_{\mathrm{ww}}\right)$, which acknowledge the dose scoring and the surrounding media as arbitrary or water. These quantities coexist to date and it is debatable which one to favour in detriment of the other. While $D_{\mathrm{mm}}$ is more likely to correlate with biological response, clinical experience on tumour, healthy tissue response and dosimetry protocols were built on $D_{\mathrm{wm}}$. Moreover, changing between quantities may yield an additional level of uncertainty.

The study presented in Chapter 5 analysed the relationship between $D_{\mathrm{wm}}$ and $D_{\mathrm{mm}}$ on small animal irradiation energies, considering the dependence of $D_{\mathrm{wm}}$ with the dose calculation approach using the intermediate cavity theory (ICT). ICT corresponds to a sum of small (SCT) and large (LCT) cavity theory contributions weighted by the parameter $\mathrm{d}$. In other words, it is the weighted sum of electronic stopping power (SCT) and mass-energy coefficient (LCT) ratios between medium and water, $\left(\overline{S_{\mathrm{el}}} / \rho\right)_{\mathrm{m}}^{\mathrm{w}}$ and $[\overline{\mu \mathrm{en}} / \rho]_{\mathrm{m}}^{\mathrm{ref}}$. The parameter $\mathrm{d}$ was calculated using two different methods, which considered the exponential attenuation of primary electrons as they enter a cavity with secondary particle transmission of $\mathrm{i} \%{ }^{[43]}$ or $4 \%{ }^{[44]}$. A set of mathematical phantoms was designed with different voxel sizes, considering the pre-clinical practice and extreme scenarios, and a selection of materials, broad enough to include the range of densities and elemental compositions conventionally used in radiotherapy, as well as an ex vivo mouse chest case with highly heterogeneous regions were included. Local photon spectra were generated in different regions of the chest case. Depending on the energy and the size of the cavity, $D_{\mathrm{mm}}$ presented lower or higher values, a factor of 0.68 to 4.37 times $D_{\text {wm }}$. 
For soft tissues, except adipose, the relationship between reporting quantities was found equal or close to unity for all different scenarios, an expected behaviour in consequence of the similarities in terms of atomic composition and density amongst the media and water, and small cross section variations. Results for adipose and bony tissues differed as their composition diverges from water. For larger cavities, where the range of secondary particles is smaller or comparable to the cavity dimensions, and for lower energies, where the predominance of photoelectric interactions is increased and highly dependent on the tissue-equivalent material compositions, the difference between dose descriptors was amplified. Hence, the impact of the cavity dimensions on estimated $D_{\mathrm{wm}}$ was significant on pre-clinical kilovolt beams up to $225 \mathrm{kV}$. Differences up to $5.1 \%$ were found between $D_{\mathrm{mm}} / D_{\mathrm{wm}}$ ratios, calculated using the local spectrum on different regions within the chest case. Therefore, the application of one single conversion factor between quantities for the whole phantom might not be appropriate. Large differences on $d$ values between the two $d$-deriving methods implied that one should be cautious when using the ICT as there is a lack of consensus on which method to use. Further simulations and measurements, explicitly tracking secondary particles are recommended for establishing a global ICT. The abiding debate over which quantity to favour is foreseen to linger while it is not clear which quantity correlates better with the biological effects of ionizing irradiation; this topic will be revisited in section 7.2.4. Finally, dose distribution comparisons should use the same reporting quantity and caution regarding reported quantity and dose comparisons is advised.

Cavity theory was originally proposed for the determination of absorbed dose in the sensitive volume (a cavity) of a detector, e.g. an ionization chamber. The conversion of absorbed dose in an air-filled cavity to absorbed dose in water was formulated by Bragg in I9I2 and Gray in 1936 and later revisited by Spencer, Attix and Burch in 1955 and Nahum in $1978^{[45-50]}$. The conditions of the Bragg-Gray (BG) theory with Spencer-Attix-Nahum stopping-power ratios formed the basis of radiation dosimetry protocols widely adopted to MV photon and electron beams ${ }^{\left[5_{1}, 52\right]}$. If the dimensions of a cavity are large relative to the range of secondary electrons e.g. for low energy $\mathrm{kV}$ beams, the BG conditions do not hold, and the mass-energy absorption coefficient ratios for cavity and medium are normally used. Although several other formalisms were proposed ${ }^{[53-56]}$, including the weighted sum used in Chapter 5, their application remained marginal as treatments with 
$\mathrm{kV}$ energies are not mainstream and MC methods emerged as a solution to the radiation transport problem allowing accurate estimation of the electron spectra within media. Revisiting this topic, regardless of powerful MC codes that calculate energy deposition in heterogeneous media, has two main justifications. First, not all small animal dose calculation engines use MC models to calculate $D_{\mathrm{mm}}$ : some algorithms ${ }^{[57-59]}$ calculate $D_{\mathrm{wm}}$ and use conversion factors to $D_{\mathrm{mm}}$. Secondly, besides dose to a water voxel (or a voxel composed of water-like tissue) in medium $D_{\mathrm{wm}}$ has been extrapolated to a biological representation of the target where energy is deposited within an organism, such as DNA-bound water molecules or a subcellular compartment whose elemental composition is closer to water than medium. As $D_{\text {wm }}$ depends on the cavity size, the significance the water cavity size in relation biological effects becomes of interest.

The cellular effects of radiation are not completely understood and the definition of radiobiological target has changed over time. Goodhead ${ }^{[60]}$ indicated that microdosimetry theories have presented putative targets on the scale of I nm up to several $\mu \mathrm{m}$. The current evidence points to $3^{-10}$ nm (initial DNA damage), o.I-O.5 $\mu \mathrm{m}$ (subsequent interactions), $\approx$ IO $\mu \mathrm{m}$ (intracellular non-targeted effects) and millimetres or more (inter-cellular bystander effects and influences of the tissue microenvironment $)^{[60]}$. Detailed track-structure simulations have assumed targets of $10-30 \mathrm{~nm}$, the DNA molecule dimensions and extra space for free radicals released in water, and have correlated a quantity corresponding to linear energy transfer (LET) with RBE ${ }^{[6,62]}$. Enger et al. ${ }^{\left[{ }_{3}\right]}$ assumed $\mu \mathrm{m}$-sized cell nuclei as the most important quantity with respect to biological effects and Thomson et al.[64] showed that doses to $\mu \mathrm{m}$-sized targets depend on the composition and geometry of surrounding structures. Oliver and Thomson ${ }^{[65,66]}$ found that $D_{\mathrm{mm}}$ in small volumes $(\approx \mathrm{IO} \mu \mathrm{m})$ is dependent on the composition and structure of the surrounding media, potentially rendering $D_{\mathrm{wm}}$ clinically meaningless until the effects of tissue composition are better understood.

Beyond the dose-reporting quantities currently employed, and besides their proportional relationship with biological effects, radiotherapy treatments and fundamental radiation knowledge could benefit from a quantity more tightly related to RBE, e.g. cell death probability, biological endpoint or treatment outcome, rather than radiation dose $e^{[67}$, ${ }^{68}$. From radiobiological and physical perspectives, as a number of factors influence the effect of radiation on cell-death (e.g. cell and radiation type, oxygenation, tissue composition, etc) it is important to understand their 
underlying relationships to be able to consider their collective impact in a clinical setting. Although in vitro testing can provide some insight, the dynamic biological characteristics of a complex living organism require well designed pre-clinical experiments to separate effects; this topic will be revisited in section $7 \cdot 2.4$.

\section{I.5 Proton beam irradiation}

In a venture into the light ion irradiation world, Chapter 6 presented an investigation on the capabilities of a clinical proton system, which features a unique adaptive aperture (AA), concerning the generations of fields small enough for pre-clinical in vivo irradiations. The in silico research was developed through a MC model of the proton beam line nozzle, which consists of an energy modulation system (EMS) and an AA. The EMS enables the $230 \mathrm{MeV}$ beam nominal range to be decreased in multiples of 2.I mm and the AA allows irradiation fields as small as $5 \mathrm{x} \mathrm{I} \mathrm{mm}^{2}$. Irradiation treatment plans were tested with a set of discrete energies, field sizes and beam directions, later compared to photon plans. Although the number of protons which hit the target drops to $\mathrm{I}-3 \%$, treatment times below five seconds were found, with good tumour coverage and less damage to the OARs than in photon plans. This study contributed to a deeper understanding of the implications regarding the degradation of the nominal $230 \mathrm{MeV}$ proton beam to low energies, the effect on spot size, dose homogeneity and dose rate.

Proton treatments in Chapter 6 were designed either using low energy fields that stop in the tumour or high energy beams to shoot through. The former allows for a decrease in dose to healthy tissues and might be used to study RBE effects. The latter could be used for small animal proton imaging, for pre-treatment position verification or to improve range uncertainty by directly measuring the animal relative stopping power maps. These two topics will be readdressed in section 7.2.4. The EMS, characterized by a constant range straggling at the Bragg peak, poses a disadvantage for pre-clinical research: it prevents an easy production of sharp beams. Furthermore, it presents an increase in lateral beam spot size for decreasing energies caused by the many plates in front of the beam. Although the AA collimates the beam, there is an intrinsic loss in efficiency when using large spot sizes with small fields: the beam is mostly absorbed by the AA. The intensity is also decreased in the central beam axis, as small angular 
deflections in proton trajectories are not negligible for small fields.

Pre-clinical studies are important means to understand the radiobiological properties of ion therapies to effectively realize their potential benefits. Although some aspects, such as the effects of cell survival and proliferationbased RBE values, have been well documented ${ }^{[69]}$, there is a lack of in vivo studies. They could be useful for different radiobiological hypothesis, e.g. RBE dependence on fractionation, differential immune effects with respect to photon beams $s^{\left[7^{0}, 7^{1}\right]}$ or, more generally, biological dependence on high LET. Ford et al..$^{\left[7^{2}\right]}$ have pointed out that irradiation technologies to study these effects are underdeveloped and that there is an urgent need for high-precision image-guided proton radiation delivery systems relevant to animal models. Currently, there are 158 clinical ion beam centres in operation, construction or planning stage ${ }^{[73]}$. Due to the high cost associated with these facilities, dedicated small animal ion beam systems are unlikely to be developed to a same extent as the photon beam ones. The proper adaptation of clinical facilities for small specimens, including image guidance, fits ideally into this context. However, challenges are expected, such as the acquisition of beam time in the clinical environment.

\subsection{Future perspectives}

\subsection{Tissue compositions}

As emphasized in section 7.I.I, tissue elemental composition reference data, heavily used on clinical and pre-clinical dose calculations, are based on a few dated studies. Novel estimation techniques could potentially increase accuracy and add information on different populations, gender and age. The non-destructive techniques particle induced $\mathrm{x}$ - and gammaray emission (PIXE and PIGE) have been suggested to be complementary used to detect heavy element trace quantities and the mass proportions of the lighter ones, respectively. In both approaches, charged particles are accelerated towards a sample of interest: they may cause ionizations and subsequent $\mathrm{x}$-ray emissions, PIXE, or, they may interact with nuclei within the sample and cause gamma-ray emissions, PIGE. Detection of these emissions may reveal the elemental composition of a sample. Additionally, destructive analytical methods include chromatography combined with mass spectrometry, infrared spectroscopy or thermal conductivity detection $^{\left[{ }_{3}\right]}$. Other techniques ex vivo such as neutron activation analysis, 
laser-induced breakdown spectroscopy (LIBS), secondary ion mass spectrometry (NanoSIMS), laser ablation-inductively coupled plasma mass spectrometry (LA-ICP-MS) have all been used for elemental composition analysis in different scopes $^{[74,75]}$.

In vivo techniques can facilitate tissue identification, based on individualized data acquisition instead of reference data. There is a multitude of techniques for composition estimation, divided into levels of increasing complexity: atomic, molecular, cellular, tissue system, and whole body ${ }^{[76]}$. They range from simple skinfold measurements to bioimpedance analysis, dual energy $\mathrm{x}$-ray absorptiometry, MRI, DECT and in vivo neutron activation analysis (IVNA) methods, such as delayed- $\gamma$, prompt $\gamma$ and inelastic neutron scattering. In IVNA methods, a subject is placed in a neutron field, its atoms might undergo nuclear reactions and release gamma rays, which are simultaneously measured by scintillation detectors and correlated to elements. Although IVNA measures whole-body compositions, tissue values can be extrapolated through empirical rules. DECT and MRI methods are more convenient and available on an individual basis and yield lower or no radiation dose. The DECT principle is mentioned in Chapter 2. MRI is based on the interaction between hydrogen nuclei (protons) and magnetic fields. Briefly, during a scan, protons absorb energy due to radiofrequency (RF) and release the absorbed energy when RF is ceased: the time it takes for protons to return to their original positions, dependent on interactions with the surrounding medium, can be exploited to yield differences between tissues.

Validation phantoms, human and animal studies for DECT and MRI have been compared to ex vivo data and showed good overall agreement within a distribution ${ }^{[77-82]}$. These studies support the validity of tissue level estimates and may be considered in vivo reference standards ${ }^{\left[8_{3}\right]}$. Yet, there are many sources of uncertainty, such as instrument calibration, stability, subject participation and observer variation. Influence of image quality parameters also play an important role. After the contribution of these factors is weighted, a distribution of values for different tissues and organs is more likely to represent anatomical structures rather than a unique mean value, producing a sound representation of tissue heterogeneity, closer to reality. It would be rather advantageous to adapt $\mathrm{MC}$ calculations to spread out or clustered values. 


\subsubsection{Image quality and dose}

Section 7.I.2 showed that a myriad of imaging protocols and algorithms could be characterized to improve image quality and possibly add more value to DECT. However, the benefits would be rather small as current CT imaging technology has inherent limitations, which yield poor intrinsic and soft tissue contrast. Although image quality is sufficient for skeletal topology, the application of contrast agents is typically necessary to visualize differences in soft tissues. This practice has its caveats as it is associated with higher experimental complexity, larger cost and dose calculations effects. To overcome these limitations, relatively new semiconductor $\mathrm{x}$-ray photon counting detectors are expected to bring CT imaging to a new technological generation.

A number of photon counting detectors are commercially available (Medipix and Timepix ${ }^{[84]}$, PILATUS ${ }^{[85]}$, Eiger ${ }^{[86]}, \mathrm{XPAD}^{[87]}$ and PiXirad ${ }^{[88]}$ ) and include a large-area option ${ }^{[89]}$ suitable for small animals ${ }^{[90]}$. They operate differently from current scintillation-based systems, which detect x-ray photons indirectly through conversion into visible light and integrate the charge from the incoming photons, whereby high energy photons end up with a stronger weight than low energy ones. The semiconductor detectors process incoming photons at the pixel level as single events and weight all photons equally, thus offering higher SNR. They feature adjustable energy thresholds which allow counting incoming photons with dedicated energy for multispectral $\mathrm{x}$-ray imaging. They are practically noiseless and CNR is limited by the number of detected photons. Consequently, soft tissue structures, with low differences in attenuation, can be visualised with high CNR ${ }^{[91]}$. Figure 7.I shows images created this technology.

Moving towards this technology has been proven feasible $e^{\text {gol }}$ and would allow for the acquisition of improved information of morphological and structural changes of individual organs or tissue structures. It can provide multiple quantitative parameters for distinguishing malignant from benign tumours ${ }^{\left[9_{2}\right]}$ and improve material analysis through the image-based extraction of tissue compositions, discussed in the previous section. Medipix detectors have been combined with mass spectrometry imaging to demonstrate spatial correlation of molecular and $3 \mathrm{D}$ structural information ${ }^{\left[{ }^{3}\right]}$. If used in place of DECT they can provide reduced motion artefacts and radiation doses, and would not require image registration. IR techniques should also be implemented for image reconstruction using 

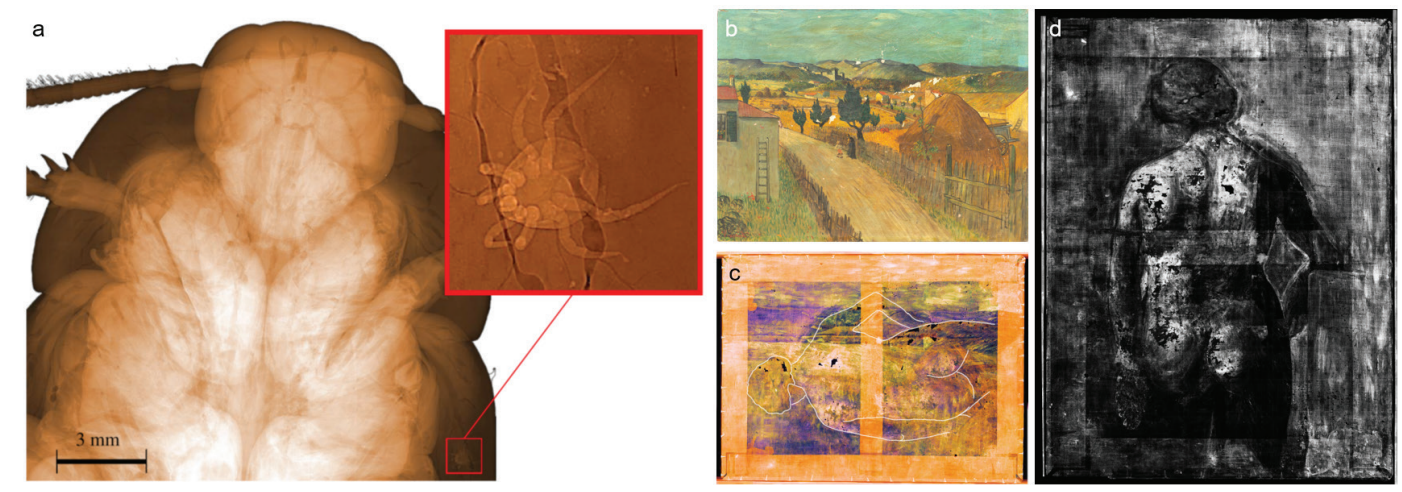

Figure 7.I - (a) Image of a cockroach taken by the WidePIX detector. The zoom detail shows veins and a tick parasite in the inset. Reproduced with kind permission from Jakubek et al. 20I4 ${ }^{[89]}$. (b-d) The use of these detectors in artworks. (b) The painting of La Crau with a View of Montmajour, with the signature Vincent, was subject to material-sensitive X-ray radiography. (c) the figure of a female nude from behind was found underneath the painting, similar to other van Gogh works. (d) The X-ray images are able to show the distribution of individual pigments across the image and the way they were applied. Using energy channel separation to achieve individual layers and materials the materials that are only related to a target layer of paint become evident. This method allows a completely new way of working with X-ray radiography using advanced pixel detectors.

this technology. Currently, the lack of a commercial system developed with hardware and software for these detectors, limit their scope to fundamental research and a few commercial applications.

In addition to hardware, image reconstruction techniques are likely to undergo substantial developments. In Chapter 3, a software platform for image reconstruction was presented. Briefly, the software received CBCT projections, encoded as an intermediate representation of the object in the detector domain, and reconstructed them into an image by the invertion of the encoding function. Analytically, it gradually solved the Radon inversion, and iteratively it used a cost function, which considered prior knowledge of projections or the image and noise properties. Image reconstruction is a challenging process: projections are acquired by imperfect and noisy detectors; iterative techniques have simplistic noise models and involve time-consuming steps. Artificial intelligence (AI) methods have been used for the entire image reconstruction process or to act on specific artefact and noise corrections.

Data-driven supervised learning frameworks have recently been proposed for image reconstruction. The automated transform by manifold approximation, AUTOMAP ${ }^{[94]}$, learns a mapping between the detector and 
the image domain, derived from training data. It is implemented with a deep neural network, which learns different reconstruction transforms for various imaging acquisition strategies, using similar network architecture and parameters. It generates images with reduced noise and artefacts when compared with conventional methods. As AUTOMAP does not handle large reconstruction matrices, the Radon inversion approximation, RAINAP ${ }^{\text {95] }}$, was proposed by a different group. It represents a unified framework for Radon inversion using neural networks. The algorithm first applies filtering and back-projection operations with two learnable appropriative network layers to perform the domain transform. Subsequently, it uses a convolutional neural network to refine the reconstruction performance. Besides algorithms targeting the reconstruction problem, several deep learning methods, including neural networks have been proposed to act on image quality parameters. For example, deep learning techniques have been used to minimize the mean-squared error between an artificially denoised CT image and the ground truth under generic penalties ${ }^{[96]}$; denoising based on the generative adversarial networks, which focus on migrating the noise distribution from statistically strong to weak, have also been proposed ${ }^{[97]}$ and convolutional neural network (CNN) or deep neural networks for sparse-view artefact reduction.

AI applied to medical imaging has been exponentially expanding and is likely to change several aspects of this field ${ }^{[98,99]}$ : it is a suitable candidate for solving ill-posed inverse problems, and, as CT scans are routinely collected, large data sets are readily available. Current commercial software does not treat image artefacts with machine learning (ML) techniques, although it is expected to happen in the near future, ML requires massive computational resources, including state-of-the-art GPUs for data training with adequate and representative data. Pre-clinically, it is expected to yield advantageous results as small animals share more similarities than humans. A proper image database is necessary and still represents a drawback as most research institutions lack an organized database. Machine learning techniques have also been proposed for noise reduction in low-dose CT, therefore reducing the subject imaging dose.

Imaging dose is a concern and a potential limiting factor if successive CT scans would influence experimental outcome. Pre-clinical irradiators have recently been adapted to support bioluminescence imaging (BLI) integrated in the same frame of reference of the CT scanner. BLI is a type of molecular imaging, where a sensitive optical camera in a light-tight 
environment records weak light sources originating up to a few centimetres deep in tissues ${ }^{[\text {roo] }}$. It has a high signal-to-noise ratio, fast acquisition times, it is easy to use and cost-effective. Optical imaging has become increasingly attractive due to the availability of targeted probes, probes activated due to enzymatic reactions or probes that can be produced by cells themselves in the form of bioluminescent enzymes ${ }^{[\text {ror }}$. It does not present additional radiation burden to the animals and can be used to evaluate longitudinal tumour growth and to estimate tumour size. However, due to low spatial resolution, typically $\mathrm{I}-2 \mathrm{~mm}$, it is not suited for morphological and somewhat suited for functional imaging ${ }^{[\mathrm{rog}]}$. It generates a two-dimensional (2D) planar image. Recent efforts have been put on $3 \mathrm{D}$ image reconstruction of this technique, which is also expected to benefit from AI techniques ${ }^{[103]}$.

\subsubsection{From target margins to treatment adaptation}

The research on target margins could spawn a couple of prospective studies. Firstly, the recipe described in Chapter 2 used DVH metrics to yield radiotherapy treatment margins. A thorough investigation on the radiosensitivity of different murine OARs based on current irradiation technology, considering dose rates and dynamic treatments could promote margin recommendations for different sites. And secondly, the effect of respiratory motion on the $\mathrm{CT}$ image blurring could be fully investigated through projection based ${ }_{4} \mathrm{D}-\mathrm{CBCT}$ simulation, using the MC code GATE, as it provides good capabilities for imaging simulation.

Another topic mentioned at the end of the discussion regarding Chapter 4 was adaptive radiotherapy. A verification system to quantify possible discrepancies between planned and delivered dose could bring extensive benefits to this field. Discrepancies with respect to planned dose can occur due to motion between imaging and treatment and due to anatomical changes in the course of treatment. The clinical practice with photon beams has adapted the imaging panel, also called electronic portal imaging device (EPID), to be a verification tool for different scenarios: before, during or after treatment and with or without the subject present during the verifications. There are four main scenarios ${ }^{[\mathrm{ro4}]}$ presented in Figure 7.2. 


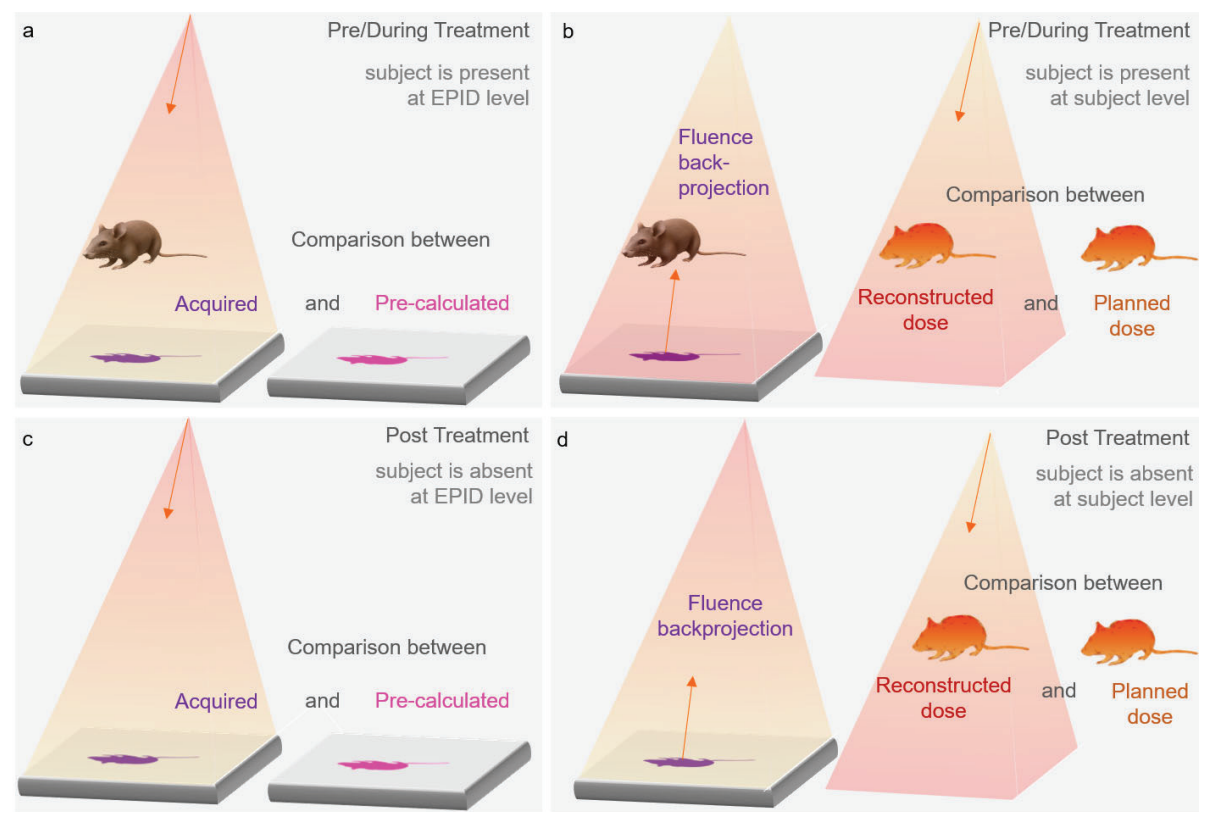

Figure 7.2 - Verification scenarios: (a) detector level with subject: an image is acquired and compared to a pre-calculated one, based on the treatment plan; (b) at subject level with subject: the photon fluence is measured at detector level after its passage through the subject and is back-projected upwards through the subject; an independent dose calculation is performed and the $3 \mathrm{D}$ reconstructed dose distribution is compared to the planned dose; (c) at detector level without subject: an image is acquired and compared to a pre-calculated one, errors related to the subject cannot be detected but it can be verified if the planned image corresponds to the delivered one and (d) at subject level without subject: fluence is backprojected without the subject and photons are subsequently forward propagated for a dose calculation in the CT image, which is compared to the planned dose.

Treatment verification implementation requires a framework for image prediction and comparison, fluence back-projection, dose reconstruction and the conversion of image into dose. Furthermore, a number of measurements and calibrations are necessary to act on possible sources of errors inherent to the image detector, such as warm-up time, radiation dose history effect, stability and short- and long-term reproducibility, gantry angle dependency, output factor and response linearity. Other sources of dose delivery errors rely on operator- and system-related factors that can individually or cumulatively contribute to dosimetric errors, such as incorrect filter selection, gantry sag and wobble, couch rotation, and collimator misalignment. Pre-clinically, dose delivery has been investigated 
using dosimeters, such as oxide semiconductor field effect transistors (MOSFETs) surgically implanted in mice or Gafchromic film for narrow $\mathrm{kV}$ beams ${ }^{[105, \text { ro6] }}$. Regarding the use of EPIDSs, Granton et al. ${ }^{[07]}$ investigated the accuracy of the treatment plan delivery using a MC model to simulate the flat panel image. Simulation was compared to measurements to verify that intended treatment dose agreed with the delivered treatment dose. Dose delivery errors resulting from intrafraction motion were also assessed. Anvari et al. ${ }^{\left[108,{ }^{109]}\right.}$ quantified the dosimetric characterization of the EPID at $\mathrm{kV} \mathrm{x}$-ray energies in a year-long period and, in a subsequent study, they converted EPID images into $2 \mathrm{D}$ dose maps at the detector plane and used these maps to calculate $2 \mathrm{D}$ dose distribution at the exit surface in phantoms. Accuracy of transit and exit dose distributions were independently validated with ion chamber and film measurements. Further development of these techniques, including $3 \mathrm{D}$ or ${ }_{4} \mathrm{D}$ models could increase treatment conformity and add more value to the comparison with or the translation to clinical practice.

\subsubsection{Dose reporting}

Previously mentioned on section 7.I.5, the debate over which dose reporting quantity to favour in radiotherapy $D_{\mathrm{mm}}$ or $D_{\mathrm{wm}}$ lacks quantitative radiobiological data. This information could potentially establish which one best correlates with biological damage. Precise radiotherapy experiments on complex living systems have only recently become feasible through novel pre-clinical irradiation platforms. Considering the opportunity to investigate a fundamental scientific question on highly controlled experimental conditions, the most immediate future work following the discoveries of this thesis should be to investigate the influence of the physical aspects on cell death caused by radiation dose, particularly the impact of dose prescribed using different quantities. A study protocol has been designed along with the radiobiology department. The aim is to analyse acute radiation effects from doses prescribed with $D_{\mathrm{mm}}$ and $D_{\mathrm{wm}}$. Several essays are foreseen, e.g. clonogenic survival, gut toxicity, blood profile and bone marrow leucocytes and lymphocytes. A range of $\mathrm{kV}$ energies should be applied, as RBE is expected to increase for lower energies, yet not excessively low as dose-rate effects should not interfere. Greater radiation damage difference is expected for lower energies. Gaining insight in this will provide essential data towards a fundamental understanding of how living tissue interacts with radiation. 


\subsubsection{Light ions}

Similar to photon platforms, clinically relevant pre-clinical proton research requires image guidance. Chapter 6 and section 7.I.5 showed the feasibility of a clinical set-up used pre-clinically and concluded that high energy beams can be used to shoot through the specimens and enable proton imaging or range verification. Proton imaging typically offers limited spatial resolution due to Coulomb scattering: on their way through matter, protons are slowed down in interactions with atomic electrons and deflected from their initial direction in electromagnetic interactions with atomic nuclei, resulting in an angular spread from many deflections. Sophisticated methods, such as single particle tracking or magnetic optics can improve image resolution ${ }^{[\mathrm{rro}]}$. Muller et al. suggested a method for dual-energy proton radiography using enhancement of residual energy distribution for different proton energies. The images show structures dominated by energy absorption or scattering of protons in the object. Although their images lack spatial resolution, they are sufficient for position verification, provide low imaging dose, use same frame of reference of the treatment beam and the absorption images allow range verification within the animal. This technique can be further exploited into proton-CT imaging.

Chapter 6 also concluded that lower energy proton treatments, designed to stop at the tumour could be used to study RBE effects. In comparison to photons, light ion beams produce increased energy deposition with penetration depth and intensify biological effectiveness with enhanced ionization density in individual particle tracks, where DNA damage becomes clustered and more difficult to repair. Radiobiological and biophysical studies have improved the RBE understanding of light ions and dose-localizing properties. However, in a recent review, Paganetti $i^{[\mathrm{mI}]}$ has challenged the whole concept of the proton RBE value of I.I. He considered it "generic, spatially invariant within tumours and normal tissues [which] disregards the evidence that proton RBE varies with LET, physiological and biological factors, and clinical endpoint". He further analysed the relationship between RBE and dose, biological endpoint and physical properties of proton beams and concluded that there are considerable uncertainties in RBE values, where additional radiobiological data is necessary to reduce uncertainties to clinically acceptable levels. Besides uncertainties in RBE, proton treatments could benefit from advanced research on fractionation schemes, radioresistance and adjuvant therapies $^{[112]}$. 
In parallel, there has been a crescent development of targeted radiopharmaceutical therapies using alpha, beta, Auger and positron emitters, due to a higher availability of isotope production. Here, instead of an external hadron beam, a pharmaceutical labelled with a radioisotope binds to specific target cells and may allow fatal dose delivery to malignant structures while sparing the majority of healthy tissue. For clinical feasibility, a radionuclide must have a suitable emission at a reasonable branching ratio, suitable chemical properties to allow attachment to relevant ligands and an appropriate half-life. This field largely relies on pre-clinical research as a pipeline for phase I clinical trials and the dosimetry is heavily based on imaging, using functional techniques such as SPECT/CT, PET/CT and autoradiography. Especially for rare radionuclides, which show promising results in animal models, such as Actinum-225 ${ }^{[\mathrm{II} 3, \mathrm{II} 4]}$ and Astatine-2II III5], it should be advantageous to research the correlation of these new techniques with standard external beam irradiation regarding treatment outcome. Moreover, research on targeted radionuclide therapy as adjuvant technique can also be beneficial. 


\subsection{Final remarks}

Regardless of the immense possibilities pre-clinical radiotherapy offers, it is unavoidable to acknowledge that small animals are not humans. Frequently, their unique characteristics favour research, e.g. ability to manipulate their phenotype and genotype. However, the vast majority of studies are not replicated in humans ${ }^{[16]}$. In a recent review, Koontz et al. ${ }^{[17]}$ pointed to some issues in successful pre-clinical translation to standard treatments, summarized in Table 7.I. Besides the difficulties in matching human and mice biology, radiation delivery and physical properties of the irradiators are often different from the clinic. Considering strategies to increase pre-clinical research productivity, they encouraged the use of orthotopic models, biomarkers, pharmacodynamic read-outs, robust imaging and computational modelling. They concluded that the selection of mice should favour "a direct bilateral link (...) to the molecular characteristics of the human condition and that of the appropriate mouse strain" and pre-clinical and clinical research should work as a cycle "considering the biology which needs to be represented in the model, tailoring the physics of the irradiation as close to the human situation as possible and acknowledging the limitations that cannot be overcome".

Finally, for successful pre-clinical research from physical and biological standpoints, the multidisciplinarity of this field cannot be forsaken. Progress can only be achieved through the combined efforts and wisdom inherent of different fields. 
Summary, discussion, and prospective work

\section{CONCERNS EXPLANATION}

biological

Treatment nave animals Mutational stress caused by heavily pre-treated human cancer

Immune status Immune system part of radiation response

Inherent biology differences Molecular biology differences between mouse and human

physical

\footnotetext{
Field Size Dose accuracy

similarity to human anatomical borders

Radiation dose/ Radiobiology differences

fractionation/dose rate

Irradiator energy $\mathrm{kV}$ planning software for accurate dose prediction differences

Animal setup OAR

Anesthesia: air mix

Radiation QA procedures Frequency of testing
}

Table 7.I - Considerations with small animal radiotherapy models. Reproduced with kind permission from Koontz et al. ${ }^{[1] 7]}$ 


\section{$7 \cdot 4$ References}

${ }^{[1]}$ F. Verhaegen, L. Dubois, S. Gianolini, M.A. Hill, C.P. Karger, K. Lauber, K.M. Prise, D. Sarrut, D. Thorwarth, C. Vanhove, B. Vojnovic, R. Weersink, J.J. Wilkens, D. Georg, ESTRO ACROP: Technology for precision small animal radiotherapy research: Optimal use and challenges, Radiother Oncol, I26 (2018) 47I-478.

${ }^{[2]}$ B.R. Walters, R. Kramer, I. Kawrakow, Dose to medium versus dose to water as an estimator of dose to sensitive skeletal tissue, Phys Med Biol, 55 (2010) 4535-4546.

${ }^{\left.{ }_{3}\right]}$ F. Verhaegen, P. Granton, E. Tryggestad, Small animal radiotherapy research platforms, Phys Med Biol, 56 (20II) R55-83.

${ }^{[4]}$ J. Bäurle, J. Kučera, S. Frischmuth, M. Lambertz, K. Kranda, Dynamics of Trace Element Concentration During Development and Excitotoxic Cell Death in the Cerebellum of Lurcher Mutant Mice, Brain Pathology, ig (2009) 586-595.

${ }^{\left.{ }_{5}\right]}$ M. Anniko, R. Wroblewski, Elemental Composition of the Developing Inner Ear, Annals of Otology, Rhinology \& Laryngology, 90 (I98I) 25-32.

${ }^{[6]}$ N. Debernardi, R.B. Roijers, R. Krams, R. De Crom, P.H.A. Mutsaers, G.J. Van Der Vusse, Microcalcifications in atherosclerotic lesion of apolipoprotein E-deficient mouse, International Journal of Experimental Pathology, 9I (2010) 485-494.

${ }^{[7]}$ J.M. Moran, D.W. Nigg, F.J. Wheeler, W.F. Bauer, Macroscopic geometric heterogeneity effects in radiation dose distribution analysis for boron neutron capture therapy, Medical Physics, I9 (1992) 723-732.

${ }^{[8]}$ M. Długaszek, K. Kopczyński, Elemental Composition of Muscle Tissue of Wild Animals from Central Region of Poland, International Journal of Environmental Research, 7 (2013) $973-978$.

$\left.{ }_{9}\right]_{\text {K. }}$ K. Buddhachat, S. Klinhom, P. Siengdee, J.L. Brown, R. Nomsiri, P. Kaewmong, C. Thitaram, P. Mahakkanukrauh, K. Nganvongpanit, Elemental Analysis of Bone, Teeth, Horn and Antler in Different Animal Species Using Non-Invasive Handheld X-Ray Fluorescence, PLoS One, II (2016) eor55458.

[ro] ICRP, Report of the Task Group on Reference Man, Pergamon Press, Oxford., ICRP Publication 23 (I975).

[rI] D.R. White, J. Booz, R.V. Griffith, J.J. Spokas, I.J. Wilson, Report 44, Journal of the International Commission on Radiation Units and Measurements, os23 (1989) NP-NP.

${ }^{\left[{ }^{2} 2\right.} \mathrm{H}$ H.Q. Woodard, D.R. White, Bone models for use in radiotherapy dosimetry, Br J Radiol, $55($ I 982$) 277-282$.

${ }^{\left[{ }_{13}\right]}$ D. Mann-Krzisnik, F. Verhaegen, S.A. Enger, The influence of tissue composition uncertainty on dose distributions in brachytherapy, Radiother Oncol, I26 (2018) 394-4IO. 
${ }^{\left.{ }^{14}\right]}$ L.E. Schyns, D.B. Eekers, B. van der Heyden, I.P. Almeida, A. Vaniqui, F. Verhaegen, Murine vs human tissue compositions: implications of using human tissue compositions for photon energy absorption in mice, Br J Radiol, DOI I0.I259/bjr.20I80454(2018) 20180454.

$\left.{ }^{\left.{ }_{15}\right]}\right]$ J.M. Boone, O. Velazquez, S.R. Cherry, Small-animal X-ray dose from micro-CT, Mol Imaging, 3 (2004) I49-I58.

${ }^{[16]}$ S.K. Carlson, K.L. Classic, C.E. Bender, S.J. Russell, Small animal absorbed radiation dose from serial micro-computed tomography imaging, Mol Imaging Biol, 9 (2007) 78-82.

${ }^{\left.{ }_{17}\right]}$ C.D. Johnstone, M. Bazalova-Carter, MicroCT imaging dose to mouse organs using a validated Monte Carlo model of the small animal radiation research platform (SARRP), Phys Med Biol, 63 (2018) II50I2.

${ }^{[18]}$ L.Y. Du, J. Umoh, H.N. Nikolov, S.I. Pollmann, T.Y. Lee, D.W. Holdsworth, A quality assurance phantom for the performance evaluation of volumetric micro-CT systems, Phys Med Biol, 52 (2007) 7087-7108.

${ }^{\left[{ }^{1}\right]}$ C.D. Johnstone, P. Lindsay, E.E. Graves, E. Wong, J.R. Perez, Y. Poirier, Y. Ben-Bouchta, T. Kanesalingam, H. Chen, A.E. Rubinstein, K. Sheng, M. Bazalova-Carter, Multiinstitutional MicroCT image comparison of image-guided small animal irradiators, Phys Med Biol, 62 (2017) 5760-5776.

${ }^{[20]}$ C.M. Ma, C.W. Coffey, L.A. DeWerd, C. Liu, R. Nath, S.M. Seltzer, J.P. Seuntjens, M. American Association of Physicists in, AAPM protocol for 40-30o kV x-ray beam dosimetry in radiotherapy and radiobiology, Med Phys, 28 (200I) 868-893.

${ }^{[21]}$ S.C.C. Klevenhagen, R.J. Aukett, R.M. Harrison, C. Moretti, A.E. Nahum, K.E. Rosser, The IPEMB code of practice for the determination of absorbed dose for $\mathrm{x}$-rays below 300 $\mathrm{kV}$ generating potential (0.035 mm Al - $4 \mathrm{~mm} \mathrm{Cu} \mathrm{HVL}$; IO - $300 \mathrm{kV}$ generating potential), Physics in Medicine and Biology, 4I (I996) 2605-2625.

${ }^{\left[{ }_{22}\right]}$ R.J. Aukett, J.E. Burns, A.G. Greener, R.M. Harrison, C. Moretti, A.E. Nahum, K.E. Rosser, I.W. Party, Addendum to the IPEMB code of practice for the determination of absorbed dose for $\mathrm{x}$-rays below $300 \mathrm{kV}$ generating potential ( $0.035 \mathrm{~mm} \mathrm{Al}_{-4} \mathrm{~mm} \mathrm{Cu} \mathrm{HVL}$ ), Phys Med Biol, 50 (2005) 2739-2748.

${ }^{[23]}$ IAEA, Implementation of the International Code of Practice on Dosimetry in Radiotherapy (TRS 398): Review of Test Results, INTERNATIONAL ATOMIC ENERGY AGENCY, Vienna, 2005.

${ }^{[24]}$ S.H. Bartling, J. Kuntz, W. Semmler, Gating in small-animal cardio-thoracic CT, Methods, 50 (2010) 42-49.

${ }^{\left.{ }^{2} 5\right]}$ M.A. Hill, B. Vojnovic, Implications of respiratory motion for small animal imageguided radiotherapy, Br J Radiol, 9o (2017) 20160482.

${ }^{[26]}$ C.T. Badea, B. Fubara, L.W. Hedlund, G.A. Johnson, 4-D Micro-CT of the Mouse Heart, Molecular Imaging, 4 (2005). 
${ }^{\left.{ }_{27}\right]}$ G. Cao, L.M. Burk, Y.Z. Lee, X. Calderon-Colon, S. Sultana, J. Lu, O. Zhou, Prospectivegated cardiac micro-CT imaging of free-breathing mice using carbon nanotube field emission x-ray, Med Phys, 37 (2010) 5306-5312.

${ }^{[28]}$ I.G. Buliev, C.T. Badea, Z. Kolitsi, N. Pallikarakis, Estimation of the heart respiratory motion with applications for cone beam computed tomography imaging: a simulation study, IEEE Transactions on Information Technology in Biomedicine, 7 (2003) 404-4II.

${ }^{[29]}$ E.B. Walters, K. Panda, J.A. Bankson, E. Brown, D.D. Cody, Improved method ofin vivorespiratory-gated micro-CT imaging, Physics in Medicine and Biology, 49 (2004) 4163$4 \mathrm{I} 72$.

${ }^{{ }_{3} 0}$ C. Badea, L.W. Hedlund, G.A. Johnson, Micro-CT with respiratory and cardiac gating, Med Phys, 3I (2004) 3324-3329.

${ }^{\left[{ }_{31}\right]}$ D. Cavanaugh, E. Johnson, R.E. Price, J. Kurie, E.L. Travis, D.D. Cody, In Vivo Respiratory-Gated Micro-CT Imaging in Small-Animal Oncology Models, Molecular Imaging, 3 (2004).

${ }^{\left[3_{2}\right]}$ N.L. Ford, H.N. Nikolov, C.J. Norley, M.M. Thornton, P.J. Foster, M. Drangova, D.W. Holdsworth, Prospective respiratory-gated micro-CT of free breathing rodents, Med Phys, 32 (2005) 2888-2898.

${ }^{\left.{ }_{33}\right]}$ L.M. Burk, Y.Z. Lee, J.M. Wait, J. Lu, O.Z. Zhou, Non-contact respiration monitoring for in-vivo murine micro computed tomography: characterization and imaging applications, Phys Med Biol, 57 (2012) 5749-5763.

${ }^{\left.{ }_{34}\right]}$ M. Drangova, N.L. Ford, S.A. Detombe, A.R. Wheatley, D.W. Holdsworth, Fast retrospectively gated quantitative four-dimensional $\left({ }_{4} \mathrm{D}\right)$ cardiac micro computed tomography imaging of free-breathing mice, Invest Radiol, 42 (2007) 85-94.

${ }^{\left.{ }_{33}\right]}$ S.H. Bartling, W. Stiller, M. Grasruck, B. Schmidt, P. Peschke, W. Semmler, F. Kiessling, Retrospective motion gating in small animal CT of mice and rats, Invest Radiol, 42 (2007) 704-7I4.

[36] N.L. Ford, A.R. Wheatley, D.W. Holdsworth, M. Drangova, Optimization of a retrospective technique for respiratory-gated high speed micro-CT of free-breathing rodents, Phys Med Biol, 52 (2007) 5749-5769.

${ }^{[37]}$ D. Ertel, Y. Kyriakou, R.M. Lapp, W.A. Kalender, Respiratory phase-correlated microCT imaging of free-breathing rodents, Phys Med Biol, 54 (2009) 3837-3846.

${ }^{[38]}$ T.H. Farncombe, Software-based respiratory gating for small animal conebeam CT, Med Phys, 35 (2008) I785-I792.

${ }_{339]}$ J. Hu, S.T. Haworth, R.C. Molthen, C.A. Dawson, Dynamic small animal lung imaging via a postacquisition respiratory gating technique using micro-cone beam computed tomography, Acad Radiol, II (2004) 96I-970. 
${ }^{\left[{ }^{\circ}\right]}$ M.A. Hill, J.M. Thompson, A. Kavanagh, I.D.C. Tullis, R.G. Newman, J. Prentice, J. Beech, S. Gilchrist, S. Smart, E. Fokas, B. Vojnovic, The Development of Technology for Effective Respiratory-Gated Irradiation Using an Image-Guided Small Animal Irradiator, Radiat Res, I88 (2017) 247-263.

${ }^{[41]}$ A.M. Frelin-Labalme, V. Beaudouin, Development of a dynamic phantom and investigation of mobile target imaging and irradiation in preclinical small animal research, Br J Radiol, 9o (2017) 20160442.

${ }^{\left[{ }_{42}\right]}$ A.M. Frelin, V. Beaudouin, C. Le Deroff, T. Roger, Implementation and evaluation of respiratory gating in small animal radiotherapy, Phys Med Biol, 63 (2018) 215024.

${ }^{[43]}$ T.E. Burlin, F.K. Chan, The effect of the wall on the Fricke dosemeter, The International Journal of Applied Radiation and Isotopes, 20 (I969) 767-775.

${ }^{\left[{ }_{44}\right]}$ A. Janssens, G. Eggermont, R. Jacobs, G. Thielens, Spectrum perturbation and energy deposition models for stopping power ratio calculations in general cavity theory, Physics in Medicine and Biology, I9 (1974) 6r9-630.

${ }^{\left[{ }_{45}\right]}$ L.H. Gray, The Absorption of Penetrating Radiation, Proceedings of the Royal Society A: Mathematical, Physical and Engineering Sciences, I22 (I929) 647-668.

${ }^{\left[{ }^{6}\right]}$ L.H. Gray, E. Rutherford, An ionization method for the absolute measurement of $\gamma$-ray energy, Proceedings of the Royal Society of London. Series A - Mathematical and Physical Sciences, 156 (I936) 578-596.

${ }^{[47]}$ L.V. Spencer, F.H. Attix, A Theory of Cavity Ionization, Radiation Research, 3 (1955).

${ }^{[48]}$ P.R.J. Burch, Cavity Ion Chamber Theory, Radiation Research, 3 (1955).

${ }^{[49]}$ P.R.J. Burch, Comment on Recent Cavity Ionization Theories, Radiation Research, 6 (I957).

${ }^{[50]}$ A.E. Nahum, Water/air mass stopping power ratios for megavoltage photon and electron beams, Physics in Medicine and Biology, 23 (I978) 24-38.

${ }^{\left[5^{1}\right]}$ P.R. Almond, P.J. Biggs, B.M. Coursey, W.F. Hanson, M.S. Huq, R. Nath, D.W. Rogers, AAPM's TG-5I protocol for clinical reference dosimetry of high-energy photon and electron beams, Med Phys, 26 (1999) I847-1870.

${ }^{\left[5_{2}\right]}$ H. Bouchard, J. Seuntjens, Chapter 4: Applications of Monte Carlo to Radiation Dosimetry, CRC Press, Boca Raton, FL, 2013.

${ }_{53}^{\left[{ }_{3}\right]}$ T.E. Burlin, A general theory of cavity ionisation, Br J Radiol, 39 (I966) 727-734.

${ }^{\text {[54] }}$ A. Janssens, The fundamental constraint of cavity theory, Physics in Medicine and Biology, 29 (I984) II57-II58.

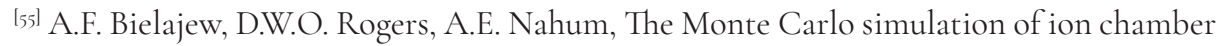
response to6oCo-resolution of anomalies associated with interfaces, Physics in Medicine and Biology, 30 (1985) 419-427. 
[56] L. Zheng-Ming, An Electron Transport Theory of Cavity Ionization, Radiation Research, 84 (1980).

${ }_{577}$ R. Jacques, J. Wong, R. Taylor, T. McNutt, Real-time dose computation: GPUaccelerated source modeling and superposition/convolution, Med Phys, 38 (20II) 294-305.

${ }^{[58]}$ N. Cho, P. Tsiamas, E. Velarde, E. Tryggestad, R. Jacques, R. Berbeco, T. McNutt, P. Kazanzides, J. Wong, Validation of GPU-accelerated superposition-convolution dose computations for the Small Animal Radiation Research Platform, Med Phys, 45 (2018) $2252-2265$.

${ }^{[59]}$ N.B. Cho, J. Wong, P. Kazanzides, Fast Inverse Planning of Beam Directions and Weights for Small Animal Radiotherapy, IEEE Transactions on Radiation and Plasma Medical Sciences, 2 (2018) 215-222.

[6o] D.T. Goodhead, Energy deposition stochastics and track structure: what about the target?, Radiat Prot Dosimetry, I22 (2006) 3-I5.

${ }^{[6]}$ D.T. Goodhead, Initial Events in the Cellular Effects of Ionizing Radiations: Clustered Damage in DNA, International Journal of Radiation Biology, 65 (1994) 7-I7.

${ }^{[62]}$ L. Lindborg, M. Hultqvist, A. Carlsson Tedgren, H. Nikjoo, Lineal energy and radiation quality in radiation therapy: model calculations and comparison with experiment, Phys Med Biol, 58 (2013) 3089-3105.

${ }^{[63]}$ S.A. Enger, A. Ahnesjo, F. Verhaegen, L. Beaulieu, Dose to tissue medium or water cavities as surrogate for the dose to cell nuclei at brachytherapy photon energies, Phys Med Biol, 57 (2012) 4489-4500.

${ }^{[64]}$ R.M. Thomson, A.C. Tedgren, J.F. Williamson, On the biological basis for competing macroscopic dose descriptors for kilovoltage dosimetry: cellular dosimetry for brachytherapy and diagnostic radiology, Phys Med Biol, 58 (20I3) II23-II50.

${ }^{[65]}$ P.A. Oliver, R.M. Thomson, A Monte Carlo study of macroscopic and microscopic dose descriptors for kilovoltage cellular dosimetry, Phys Med Biol, 62 (2017) I4I7-I436.

[66] P.A.K. Oliver, R.M. Thomson, Cavity theory applications for kilovoltage cellular dosimetry, Phys Med Biol, 62 (2017) 4440-4459.

${ }^{\left[{ }_{67}\right]}$ M. Podesta, Time dependent verification of dynamic external beam radiotherapy, School for Oncology and Developmental Biology, Radiotherapy Research, Maastricht University, Maastricht, 2016, pp. I70.

${ }^{[68]}$ M.M. Ahmed, C.N. Coleman, M. Mendonca, S. Bentzen, B. Vikram, S.M. Seltzer, D. Goodhead, C. Obcemea, R. Mohan, K.M. Prise, J. Capala, D. Citrin, G. Kao, M. Aryankalayil, I. Eke, J.C. Buchsbaum, P.G.S. Prasanna, F.F. Liu, Q.T. Le, B. Teicher, D.G. Kirsch, D. Smart, J. Tepper, S. Formenti, D. Haas-Kogan, D. Raben, J. Mitchell, Workshop Report for Cancer Research: Defining the Shades of Gy: Utilizing the Biological Consequences of Radiotherapy in the Development of New Treatment Approaches-Meeting Viewpoint, Cancer Res, 78 (2018) 2166-2170. 
${ }^{[69]}$ H. Paganetti, A. Niemierko, M. Ancukiewicz, L.E. Gerweck, M. Goitein, J.S. Loeffler, H.D. Suit, Relative biological effectiveness (RBE) values for proton beam therapy, International Journal of Radiation Oncology*Biology*Physics, 53 (2002) 407-42I.

[7o] B. Jones, R.G. Dale, Estimation of optimum dose per fraction for high LET radiations: Implications for proton radiotherapy, International Journal of Radiation Oncology*Biology*Physics, 48 (2000) I549-I557.

${ }^{[71}$ S. Girdhani, R. Sachs, L. Hlatky, Biological effects of proton radiation: what we know and don't know, Radiat Res, 179 (2013) 257-272.

${ }_{\left.77^{2}\right]}$ E. Ford, R. Emery, D. Huff, M. Narayanan, J. Schwartz, N. Cao, J. Meyer, R. Rengan, J. Zeng, G. Sandison, G. Laramore, N. Mayr, An image-guided precision proton radiation platform for preclinical in vivo research, Phys Med Biol, 62 (2017) 43-58.

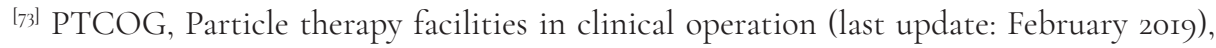
2018.

${ }_{774}$ B. Busser, S. Moncayo, J.-L. Coll, L. Sancey, V. Motto-Ros, Elemental imaging using laser-induced breakdown spectroscopy: A new and promising approach for biological and medical applications, Coordination Chemistry Reviews, 358 (2018) 70-79.

${ }^{175]}$ J. Nunez, R. Renslow, J.B. Cliff, 3rd, C.R. Anderton, NanoSIMS for biological applications: Current practices and analyses, Biointerphases, I3 (2017) 03Bzor.

${ }^{\text {[76] }}$ M.O. Fosbol, B. Zerahn, Contemporary methods of body composition measurement, Clin Physiol Funct Imaging, 35 (2015) 8I-97.

${ }^{[77]}$ N. Abate, D. Burns, R.M. Peshock, A. Garg, S.M. Grundy, Estimation of adipose tissue mass by magnetic resonance imaging: validation against dissection in human cadavers, Journal of Lipid Research, 35 (1994) I490-I496.

${ }^{\left[7^{81}\right.}$ A. Aliprantis, J. Wang, R.N. Baumgartner, R.N. Pierson, Jr, S. Lichtman, S.B. Heymsfield, Y. Kamen, Body composition of humans: comparison of two improved four-compartment models that differ in expense, technical complexity, and radiation exposure, The American Journal of Clinical Nutrition, $5^{2}$ (I990) 52-58.

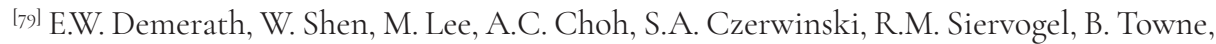
Approximation of total visceral adipose tissue with a single magnetic resonance image, Am J Clin Nutr, 85 (2007) 362-368.

${ }^{[80]}$ M. Patino, A. Prochowski, M.D. Agrawal, F.J. Simeone, R. Gupta, P.F. Hahn, D.V. Sahani, Material Separation Using Dual-Energy CT: Current and Emerging Applications, Radiographics, 36 (2016) I087-II05.

${ }^{\left[\mathrm{s}_{\mathrm{r}}\right]}$ G. Landry, K. Parodi, J.E. Wildberger, F. Verhaegen, Deriving concentrations of oxygen and carbon in human tissues using single-and dual-energy CT for ion therapy applications, Phys Med Biol, 58 (2013) 5029-5048. 
${ }^{\left[{ }^{82}\right]}$ G. Landry, P.V. Granton, B. Reniers, M.C. Ollers, L. Beaulieu, J.E. Wildberger, F. Verhaegen, Simulation study on potential accuracy gains from dual energy CT tissue segmentation for low-energy brachytherapy Monte Carlo dose calculations, Phys Med Biol, 56 (201I) 6257-6278.

${ }^{\left[8_{3}\right]}$ S.B. Heymsfield, Z. Wang, R.N. Baumgartner, R. Ross, Human body composition: advances in models and methods, Annu Rev Nutr, I7 (1997) 527-558.

${ }^{\left[{ }^{84}\right]}$ X. Llopart, R. Ballabriga, M. Campbell, L. Tlustos, W. Wong, Timepix, a 65k programmable pixel readout chip for arrival time, energy and/or photon counting measurements, Nuclear Instruments and Methods in Physics Research Section A: Accelerators, Spectrometers, Detectors and Associated Equipment, 58I (2007) 485-494.

${ }^{\left[{ }_{5}\right]}$ B. Henrich, A. Bergamaschi, C. Broennimann, R. Dinapoli, E.F. Eikenberry, I. Johnson, M. Kobas, P. Kraft, A. Mozzanica, B. Schmitt, PILATUS: A single photon counting pixel detector for X-ray applications, Nuclear Instruments and Methods in Physics Research Section A: Accelerators, Spectrometers, Detectors and Associated Equipment, 607 (2009) $247-249$.

${ }^{[86]}$ R. Dinapoli, A. Bergamaschi, B. Henrich, R. Horisberger, I. Johnson, A. Mozzanica, E. Schmid, B. Schmitt, A. Schreiber, X. Shi, G. Theidel, EIGER: Next generation single photon counting detector for X-ray applications, Nuclear Instruments and Methods in Physics Research Section A: Accelerators, Spectrometers, Detectors and Associated Equipment, 650 (2011) 79-83.

$\left.{ }^{\left[{ }_{7}\right]}\right]$ J.F. Berar, N. Boudet, P. Breugnon, B. Caillot, B. Chantepie, J.C. Clemens, P. Delpierre, B. Dinkespiller, S. Godiot, C. Meessen, M. Menouni, C. Morel, P. Pangaud, E. Vigeolas, S. Hustache, K. Medjoubi, XPAD3 hybrid pixel detector applications, Nuclear Instruments and Methods in Physics Research Section A: Accelerators, Spectrometers, Detectors and Associated Equipment, 607 (2009) 233-235.

${ }^{[88]}$ R. Bellazzini, G. Spandre, A. Brez, M. Minuti, M. Pinchera, P. Mozzo, Chromatic X-ray imaging with a fine pitch CdTe sensor coupled to a large area photon counting pixel ASIC, Journal of Instrumentation, 8 (2013) Co2O28-Co2O28.

${ }^{\left[{ }^{8}\right]}$ J. Jakubek, M. Jakubek, M. Platkevic, P. Soukup, D. Turecek, V. Sykora, D. Vavrik, Large area pixel detector WIDEPIX with full area sensitivity composed of Ioo Timepix assemblies with edgeless sensors, Journal of Instrumentation, 9 (2014) Co4Or8-Co4Or8.

${ }^{\left[{ }_{90}\right]}$ J. Dudak, J. Zemlicka, F. Krejci, S. Polansky, J. Jakubek, J. Mrzilkova, M. Patzelt, J. Trnka, X-ray micro-CT scanner for small animal imaging based on Timepix detector technology, Nuclear Instruments and Methods in Physics Research Section A: Accelerators, Spectrometers, Detectors and Associated Equipment, 773 (2015) 8I-86.

${ }^{\left[{ }_{\mathrm{r}}\right]}$ J. Dudak, J. Zemlicka, J. Karch, M. Patzelt, J. Mrzilkova, P. Zach, Z. Hermanova, J. Kvacek, F. Krejci, High-contrast X-ray micro-radiography and micro-CT of ex-vivo soft tissue murine organs utilizing ethanol fixation and large area photon-counting detector, Sci Rep, 6 (2016) 30385. 
${ }^{\left.{ }_{92}\right]}$ M.-L. Chen, X.-T. Li, Y.-Y. Wei, L.-P. Qi, Y.-S. Sun, Can spectral computed tomography imaging improve the differentiation between malignant and benign pulmonary lesions manifesting as solitary pure ground glass, mixed ground glass, and solid nodules?, Thoracic Cancer, Iо (2019) 234-242.

${ }^{\left.{ }_{93}\right]}$ E.S. E Schioppa Jr, Jr., S. Ellis, A.L. Bruinen, J. Visser, R.M.A. Heeren, J. Uher, E. Koffeman, Combined X-ray CT and mass spectrometry for biomedical imaging applications, Journal of Instrumentation, 9 (2014) $\mathrm{CO}_{4} \mathrm{O} 29-\mathrm{Co} 4 \mathrm{O} 29$.

${ }^{\left.{ }_{94}\right]}$ B. Zhu, J.Z. Liu, S.F. Cauley, B.R. Rosen, M.S. Rosen, Image reconstruction by domaintransform manifold learning, Nature, 555 (2018) 487-492.

${ }^{[95]}$ J. He, J. Ma, Radon Inversion via Deep Learning, arXiv e-prints, 2018.

${ }^{\left[{ }^{6}\right]}$ Y. Ge, Q. Zhang, Z. Hu, J. Chen, W. Shi, H. Zheng, D. Liang, Deconvolution-Based Backproject-Filter (BPF) Computed Tomography Image Reconstruction Method Using Deep Learning Technique, arXiv e-prints, 2018.

${ }_{977}^{[9}$ Q. Yang, P. Yan, Y. Zhang, H. Yu, Y. Shi, X. Mou, M.K. Kalra, Y. Zhang, L. Sun, G. Wang, Low-Dose CT Image Denoising Using a Generative Adversarial Network With Wasserstein Distance and Perceptual Loss, IEEE Trans Med Imaging, 37 (2018) I348-I357.

${ }^{[98]}$ F. Pesapane, M. Codari, F. Sardanelli, Artificial intelligence in medical imaging: threat or opportunity? Radiologists again at the forefront of innovation in medicine, European Radiology Experimental, 2 (2018).

${ }^{\left.{ }_{99}\right]}$ A. Hosny, C. Parmar, J. Quackenbush, L.H. Schwartz, H.J.W.L. Aerts, Artificial intelligence in radiology, Nat Rev Cancer, I8 (2018) 500-5IO.

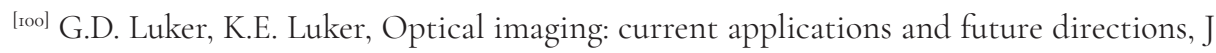
Nucl Med, 49 (2008) I-4.

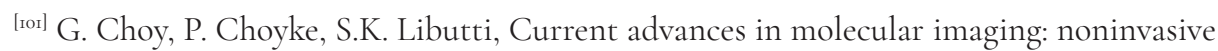
in vivo bioluminescent and fluorescent optical imaging in cancer research, Mol Imaging, 2 (2003) 303-3I 2 .

${ }^{[102]}$ K. O’Neill, S.K. Lyons, W.M. Gallagher, K.M. Curran, A.T. Byrne, Bioluminescent imaging: a critical tool in pre-clinical oncology research, J Pathol, 220 (2010) 317-327.

${ }^{\left[{ }^{\mathrm{rog}}\right]}$ Y. Gao, K. Wang, Y. An, S. Jiang, H. Meng, J. Tian, Nonmodel-based bioluminescence tomography using a machine-learning reconstruction strategy, Optica, 5 (2018).

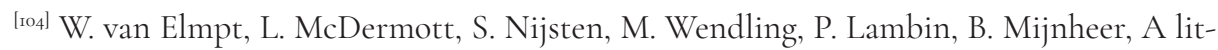
erature review of electronic portal imaging for radiotherapy dosimetry, Radiother Oncol, 88 (2008) 289-309.

${ }^{\left[{ }^{105}\right]}$ W. Ngwa, H. Korideck, L. Chin, M. Makrigiorgos, R. Berbeco, SU-E-T-3I7: MOSFET Assessment of Radiation Dose Delivered to Mice Using the Small Animal Radiation Research Platform (SARRP), Medical Physics, 38 (2011) 3560-3560. 


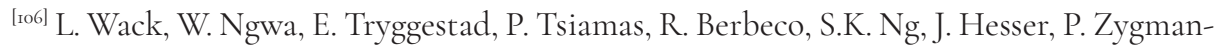
ski, High throughput film dosimetry in homogeneous and heterogeneous media for a small animal irradiator, Phys Med, 30 (2014) 36-46.

${ }^{\left[{ }^{107}\right]}$ P.V. Granton, M. Podesta, G. Landry, S. Nijsten, G. Bootsma, F. Verhaegen, A combined dose calculation and verification method for a small animal precision irradiator based on onboard imaging, Med Phys, 39 (2012) 4I55-4166.

${ }^{\left[{ }_{\mathrm{ros}}\right]}$ A. Anvari, Y. Poirier, A. Sawant, Development and implementation of EPID-based quality assurance tests for the small animal radiation research platform (SARRP), Med Phys, 45 (2018) 3246-3257.

${ }^{\text {[rogl }}$ A. Anvari, Y. Poirier, A. Sawant, Kilovoltage transit and exit dosimetry for a small animal image-guided radiotherapy system using built-in EPID, Med Phys, 45 (2018) 4642-465I.

${ }^{[\text {[п] }}$ M. Prall, M. Durante, T. Berger, B. Przybyla, C. Graeff, P.M. Lang, C. LaTessa, L. Shestov, P. Simoniello, C. Danly, F. Mariam, F. Merrill, P. Nedrow, C. Wilde, D. Varentsov, High-energy proton imaging for biomedical applications, Sci Rep, 6 (2016) 2765I.

${ }^{[\text {[rI] }}$ H. Paganetti, Relative biological effectiveness (RBE) values for proton beam therapy. Variations as a function of biological endpoint, dose, and linear energy transfer, Phys Med Biol, 59 (2014) R4I9-472.

${ }^{[12]}$ E. Fokas, G. Kraft, H. An, R. Engenhart-Cabillic, Ion beam radiobiology and cancer: time to update ourselves, Biochim Biophys Acta, I796 (2009) 216-229.

${ }^{\left[{ }_{1}\right]}$ P.E. Borchardt, R.R. Yuan, M. Miederer, M.R. McDevitt, D.A. Scheinberg, Targeted Actinium-225 $<$ strong $><\mathrm{em}>$ in Vivo $</ \mathrm{em}></$ strong $>$ Generators for Therapy of Ovarian Cancer, Cancer Research, 63 (2003) 5084-5090.

${ }^{\left[{ }_{114}\right]}$ M.R. McDevitt, G. Sgouros, R.D. Finn, J.L. Humm, J.G. Jurcic, S.M. Larson, D.A. Scheinberg, Radioimmunotherapy with alpha-emitting nuclides, Eur J Nucl Med, 25 (I998) I34I-I35I.

${ }^{\left[{ }_{15}\right]}$ J.J. Orozco, T. Back, A. Kenoyer, E.R. Balkin, D.K. Hamlin, D.S. Wilbur, D.R. Fisher, S.L. Frayo, M.D. Hylarides, D.J. Green, A.K. Gopal, O.W. Press, J.M. Pagel, Anti-CD 45 radioimmunotherapy using (2II)At with bone marrow transplantation prolongs survival in a disseminated murine leukemia model, Blood, I2I (2013) 3759-3767.

${ }^{[1,6]}$ S.E. Gould, M.R. Junttila, F.J. de Sauvage, Translational value of mouse models in oncology drug development, Nature Medicine, 2I (2015) 43 I.

${ }^{\left[{ }_{1}\right]}$ B.F. Koontz, F. Verhaegen, D. De Ruysscher, Tumour and normal tissue radiobiology in mouse models: how close are mice to mini-humans?, Br J Radiol, 90 (2017) 2016044I. 
Summary, discussion, and prospective work 



\section{LIST OF ABBREVIATIONS}

$3^{\mathrm{D}}$

${ }_{4} \mathrm{D}$

AA

AAPM

AI

AP

BG

BLI

$\mathrm{C}_{3}-\mathrm{C} 8$

CBCT

$\mathrm{CG}$

CNR

CPE

CSDA

CT

CTV

D5/90/95

DECT

$\operatorname{DECT}_{7,8,9}$

$\delta$

$\delta$-eqm

DICOM

Dmm

DNA

DVH

Dwm

Dww

EBRT

ECG

ECUT

EGSnrc

EMS

EPID

ESF
Three-dimensional

Four-dimensional

Adaptive aperture

The American Association of Physicists in Medicine

Artificial intelligence

Anterior-posterior

Bragg-Gray

Bioluminescence Imaging

Circular collimators with diameters ranging from 3-8 mm

Cone-beam computed tomography

Conjugate gradient

Contrast-to-noise ratio

Charged particle equilibrium

Continuous slowing down approximation

Computed tomography

Clinical target volume

Dose to the $5 \% / 90 \% / 95 \%$ of the tumour volume

Dual energy computed tomography

Dual-energy CT segmented with seven, eight or nine materials cut-off energy of an electron that has a range just sufficient to cross the cavity

$\delta$-ray equilibrium

Digital imaging and communications in medicine

Dose-to-medium-in-medium

Deoxyribonucleic acid

Dose-volume histogram

Dose-to-water-in-medium

Dose-to-water-in-water

External beam radiation therapy

Electrocardiogram

Electron cutoff energy

Electron Gamma Shower (National Research Council of Canada)

Energy modulation system

Electronic portal imaging device

Edge spread function

ESTRO ACROP European society for radiotherapy and oncology advisory

committee in radiation oncology practice

FBP

Filtered back-projection

FDK Feldkamp-Davis-Kress (algorithm)

FFDL(R) Feet first decubitus left (right) 


\begin{tabular}{ll} 
FFP(S) & Feet first prone (supine) \\
FWHM & Full-width half maximum \\
GATE & Geant 4 Application for Tomographic Emission \\
GPU & Graphics processing units \\
GTV & Gross target volume \\
HFDL(R) & Head first decubitus left (right) \\
HFP(S) & Head first prone (supine) \\
HU & Hounsfield unit \\
HUH & High-energy scan \\
HUL & Low-energy scan \\
ICRP & International Commission on Radiological Protection \\
ICRU & International Commission on Radiation Units and Measurements \\
ICT & Intermediate cavity theory \\
IDD & Integrated depth doses \\
IR & Iterative reconstruction \\
IVNA & In vivo neutron activation analysis \\
kV & Kilovolt \\
kVp & Kilovolt peak \\
LA-ICP-MS & Laser ablation-inductively coupled plasma mass spectrometry \\
LCT & Large cavity theory \\
LET & Linear energy transfer \\
LIBS & Laser-induced breakdown spectroscopy \\
LSF & Line spread function \\
mA & Miliampere \\
mAs & Miliampere-second \\
\hline$\mu_{\text {en }} / \rho$ & Mass-energy absorption coeeficient \\
MBDC & Model-based dose calculation \\
MC & Monte Carlo \\
MeV & Megaelectronvolt \\
MHA/MHD & Insight Meta-Image (MetaHeaDer All-in-one) \\
ML & Machine learning \\
MOBY & 4-D Mouse whole body \\
MR(I) & Magnetic resonance (imaging) \\
MTF & Modulation transfer function \\
MV & Megavolt \\
NA & Avogadro's number \\
NanoSIMS & Nano-secondary ion mass spectrometry \\
NTCP & Normal tissue complication probability \\
NURBS & Non-uniform rational basis splines \\
OAR & Organ-at-risk \\
&
\end{tabular}




$\begin{array}{ll}\text { PI-4 } & \text { (tumour) Positions I-4 } \\ \text { PCUT } & \text { Photon cutoff energy } \\ \text { PET } & \text { Positron emission tomography } \\ \text { PIGE } & \text { Particle induced x-ray emission } \\ \text { PIXE } & \text { Particle induced gamma ray emission } \\ \text { PTV } & \text { Planning target volume } \\ \text { QA } & \text { Quality assurance } \\ \text { RBE } & \text { Relative biological effectiveness } \\ \text { RF } & \text { Radiofrequency } \\ \rho & \text { Mass density } \\ \rho_{\text {C }} & \text { calculated relative electron density } \\ \rho_{\text {e }} & \text { relative electron density } \\ \rho_{\mathrm{R}} & \text { reference relative electron density } \\ \text { ROI } & \text { Region of interest } \\ \text { RS } & \text { Reconstruction software } \\ \text { RT } & \text { Radiotherapy } \\ \text { RTK } & \text { Reconstruction toolkit } \\ \text { SA } & \text { Spencer-Attix theory } \\ \text { SAD } & \text { Source-axis distance } \\ \text { SART } & \text { Simultaneous algebraic reconstruction technique } \\ \text { SCT } & \text { Small cavity theory } \\ \text { SE } & \text { Single energy } \\ \text { SECT } & \text { Single-energy computed tomography } \\ \text { SECT3,4,7 } & \text { Single-energy computed tomography segmented with three, } \\ & \text { four or seven materials } \\ \text { SECT50,90 } & \text { Single-energy computed tomography at 50, 9o kilovolts } \\ \text { SIRT } & \text { Simultaneous iterative reconstruction technique } \\ \text { SNR } & \text { Signal to noise ratio } \\ \text { SPECT } & \text { Single photon emission tomography } \\ \text { SSD } & \text { Source-to-surface distance } \\ \left(S_{\text {el }}(E)\right) \rho & \text { Electronic stopping power } \\ \text { tI-6 } & \text { Treatment scenarios I-6 } \\ \text { TCP } & \text { Tumour control probability } \\ \text { TFI-3 } & \text { Treatment fields I-7 } \\ \text { TG } & \text { Task group } \\ \text { TOPAS } & \text { TOolkit for PArticle Simulation } \\ \text { TPS } & \text { Treatment planning system } \\ \text { VIO,90 } & \text { Percentage of tumour volume which receives a dose equal or } \\ \text { VOI } & \text { Volume of interest } \\ \text { VOXSI } & \text { VOXelized CT SImulator } \\ \text { Z } & \text { Atomic number } \\ & \end{array}$


$Z_{C} \quad$ Calculated effective atomic number

$Z_{\text {eff }} \quad$ Effective atomic number

$Z_{\mathrm{R}}$

Reference effective atomic number 



\section{Social impact and valorisation}

\section{Relevance: societal impact}

Cancer is a leading cause of mortality and morbidity worldwide: one in five men and one in six women develop cancer during their lifetime [GLOBOCAN, 20I8]. The affected population can be much higher if besides the patient, the family and social circle are considered to be dealing with the disease. The main therapy options consist of surgery, chemotherapy, radiotherapy or combination strategies. For many cancer types, such strategies succeed in prolonging life, but survival remains poor due to disease relapses and treatment failure. Radiotherapy, the focus of this thesis, aims to deliver a sufficiently high dose of ionizing radiation to the tumour while sparing surrounding healthy tissues as much as possible. Chapter I describes how, historically, therapies evolved in an urgent fashion: as novel treatment strategies were critical, they were not always based on randomized clinical trials, biological models or epidemiological studies and, currently, as technology evolves swiftly and yields probable patient benefit, the shift towards sophisticated conformal therapies has also not been thoroughly validated on animal models or human trials.

In this scenario, pre-clinical research platforms provide a cost-effective experimental pipeline for timely validation of new treatment modalities or interpretation of clinical experience on large cohorts. This link is essential for innovation in cancer care and to unravel fundamental questions of radiotherapy, thus, directly impacting a vast fraction of the society. Investing on high quality pre-clinical radiotherapy amplifies research scope, minimizes study costs, animal usage and increases the likelihood of clinical translation. 


\section{Products and innovation}

In this thesis different strategies for increasing quality and value of preclinical radiotherapy were shown.

\section{Imaging}

Imaging is pivotal in radiotherapy as treatment plans are developed based on information retrieved from CT scans. Particular to small animal irradiation is the requirement for decreased spatial resolution due to size of the specimens investigated. Our group pioneered the development of dualenergy CT techniques for pre-clinical radiotherapy. A simple and effective method to retrieve atomic composition from CT images was developed and thus generated another layer of information on top of the regular tissue densities present on CT scans. Throughout this thesis the added value of this technique resonates: it improves tissue segmentation, decreases dose calculation errors and ultimately helps to ensure that the simulation and treatment doses coincide. The latter is a crucial point as the translation of research to the clinic is dependent on precise irradiation regimens.

A software platform for pre-clinical cone-beam CT image reconstruction using analytical and iterative techniques is an important product of this research. This software has been developed based on open-source libraries and published algorithms. While commercial solutions provide only limited analytical reconstruction software prone to noise and artefacts, our platform allows for image reconstruction using different algorithms, filters, artefact reduction kernels and it is likely to produce images of superior quality. The software can potentially be distributed or commercialized among other small animal irradiator users and contribute to improving image quality throughout this field.

\section{Irradiation}

A tool for defining optimal irradiation collimators for lung tumours is another product of this research. Software was developed to determine irradiation margins, based on the specimen breathing function and the available collimators of the machine. Although the concept of irradiation margins is essential for clinical radiotherapy, in the pre-clinical field it is still incipient. This was the first research to challenge this concept and propose 
a solution in the shape of a recipe, considering anatomy and hardware. As this research was first presented at the 4 th Conference on Small Animal Precision Image-Guided Radiotherapy, in Lisbon, 2018, it was awarded a prize, which is an important measurement for its relevance.

A contribution has been given to the light ion field, following the great expansion of this radiotherapy technique in the Netherlands and worldwide. Although proton therapy can potentially decrease short and long term secondary effects of radiation in the human body there is still little evidence favouring proton in comparison to photon therapy. As building costs for dedicated research facilities are prohibitive, the adaptation of a clinical set-up for pre-clinical irradiation was investigated and proved feasible within very short irradiation times. This is an important result as it enables research on biological effects of proton beams, comparison between treatment strategies and adjuvant therapies, development of new imaging modalities and gathering of evidence regarding the benefits of this technique.

\section{Dose concept}

A final contribution of this research challenges differences between analytical dose calculation algorithms (clinically and pre-clinically available), Monte Carlo techniques and the concept of dose to water and dose to medium. Using the two quantities, for kilovolt energies, large dose differences were found for tissues which differ from water in terms of elemental compositions e.g. adipose and bone. Because the different calculation methods coexist in practice and for the last two decades the scientific community has not agreed on which quantity should prevail, this research brings evidence to pursue in-vivo experiments as ultimately one dose quantity brings a higher biological damage. This research has a direct clinical effect as worldwide thousands of patients are treated daily with kilovolt treatments. 
The last decennium saw the rise of two pre-clinical radiation research cabinet vendors, Precision X-Ray Inc (USA) and XStrahl ltd (UK), which brought unprecedented image guidance and enabled precise animal irradiation. Currently, there are over I30 irradiators from both companies worldwide, each of which may cost well above half a million euros. Despite the lavish prices, these irradiators were not initially supplied with commercial software, forcing users to limit their potential scope or to invest in in-house solutions. Thus, in parallel, a spin-off company was established to act on these shortcomings, providing solutions to users of different backgrounds, e.g. SmART Scientific Solutions BV (Maastricht, the Netherlands), and have since expanded their sphere of influence. Lastly, partially due to the expansion of pharmaceutical and clinical research organizations into the pre-clinical field, imaging modalities with a clinical analogue e.g. MRI, CT, PET, SPECT, optical fluorescence or modalities unique to pre-clinical studies e.g. optical bioluminescence, intravital microscopy and phase contrast optical imaging have incorporated the most recent advances in technology to e.g. provide sub-millimetre resolution and quantification. The cost associated with these techniques can range from tens of thousands for fluoroscopy to over a million for a state-of-the-art MRI facility. The small animal imaging market is estimated to reach 2.5 billion dollars by 2025 [source: iHealthcareAnalyst, Inc., Sep. 9, 2019].

The pre-clinical market is in expansion which increasingly affects different medical and pharmaceutical fields. On the irradiation side, so far, research has mainly focused on radiation therapy for cancer treatment using photons. It is a broad field which continuously absorbs new technologies and requires experimenting to unfold underlying physical, chemical and biological mechanisms. The irradiators are also a platform for research on radiation as a therapy agent for diseases other than cancer, such as brain malformations, epilepsy, aneurysm and regenerative medicine and, in the pharmaceutical field, to test the benefit of agents from chemotherapy, immunotherapy, gene therapy or targeted therapy in combination with or compared to radiotherapy. The imaging segment is an invaluable component of modern biomedical research due to its clinically translatable, non-invasive and quantitative nature. It provides means of serially assaying biological structure and function, from oncological (or cardiovascular, neurological, etc.) disease inception to progression, and monitoring treatment effectiveness. It is employed in several fields, for treatment 
monitoring, novel therapeutic strategies evaluation and drug development. Quantitative imaging is likely to accelerate drug discovery and provide earlier and more clinically meaningful assays of therapeutic response, thereby avoiding unnecessary toxicities, expense and loss of time.

As photon irradiation may not be the ideal option for many cases, there are ongoing major investments into proton and ion irradiation clinical treatment centres. In July 20I9, there were I62 particle therapy facilities worldwide (44 under construction, 23 planned and 95 operational), however very few pre-clinical commercial (or in-house) solutions. This field requires extensive pre-clinical investigation to prove and promote the added benefit of particle therapy thus, it is expected to present substantial growth in the next decade. 


\section{4. $\quad$ Future}

The irradiation cabinet vendors who transformed the last decade of preclinical research with novel precision techniques did not however continue to invest or inject new technologies into the field. Despite the elevated costs, the cabinets are bulky and relatively simple: an X-ray tube and a flat panel detector with the dual purpose of imaging and irradiation. Using these machines for testing new therapy strategies, such as dose-painting, despite technologically feasible is however impractical due to a cumbersome process. Hence there is still a large gap between clinical and pre-clinical radiotherapy research possibilities.

A new generation of devices is expected to arise in the following years to tackle the current deficiencies of the market. The integration of novel hardware and software to the existing irradiators and the rise of original concepts is forecast to shorten the distance to the clinical practice. The incorporation of photon counting detectors to irradiation and imaging is the major predicted innovation in the near future. Such solid-sate detectors, mentioned in Chapter 7 , are able to acquire individual photon information regarding the point of entry, energy, orientation, and time of flight, which allows for several imaging techniques e.g. spectral CT, X-ray fluorescence, PET, SPECT from a range of beams e.g. photons, protons, ions, within the same device.

A rise of commercial solutions for ion beam therapy, as dedicated or modular devices to be linked to the clinical setups is currently necessary for particle therapy biological evaluation and also heavily expected in the near future. Moreover, simple approaches to daily issues as motion mitigation, dose guidance, position verification and automatic collimation techniques are expected to be more broadly implemented. Finally, artificial intelligence techniques for imaging, segmentation, planning, dose calculation and dose verification are expected to find a favourable environment in the preclinical world as similarities among animal specimens are increased when compared to their human counterparts and as such techniques are likely to contribute to the philosophy of replacement, reduction and refinement, promoting animal welfare. 



\section{Acknowledgements}

Looking back on the extraordinary journey this $\mathrm{PhD}$ has been, I feel overwhelmed with gratitude and joy in humbly trying to identify all the different individuals who have lent me their shoulders to stand on. As most of my journeys, this was not a linear nor plain one. It started in Ireland (or, in reality, in Brazil passing through Belgium and Italy) and finished in the Netherlands. It began as a clinical project on dose-guided radiotherapy and ended as many of the different flavours of pre-clinical radiotherapy physics. It was supposed to be a project calmly developed in the course of four years but became the challenge of achieving excellence by publishing at least four research pieces as main author in two years. Finally, it started with a person who thought she had a good scientific knowledge and who now knows she has a lot learn, but is eager to continuing pursuing scientific research. Reaching this point was only possible through the guidance and rapport with my supervisors.

Frank, you gave me an opportunity (when I really needed it), believed in my work and guided me through it. You gave me a platform to establish myself professionally, to develop my scientific judgment and to produce interesting and even fundamental research. Your exceptional availability, the fair and equal treatment you give everyone and your acknowledgement on individual contributions have always stimulated my enthusiasm towards work. I felt it was possible to ask for advice, help, I learned to be more critical, creative and to present my work more objectively. Through you I also gladly met and collaborated with Brigitte. It has been a superb experience to which I am thankful and I hope we can continue collaborating.

Gabriel, you were fundamental for my PhD. You practically did not know me when I first came to the Netherlands, and yet you offered me a place (for free) in your house. But that is just the kind of person you are. No matter how busy or stressed, you always found time to patiently listen, give constructive feedback and actively help everyone. You are a great professor, researcher (although you were awarded an engineering prize) and a splendid person. It makes me happy to be able to share my experiences and the memories of my PhD with you and Louise (and Planck and Pinta). 
Ludwig, I have always admired you as a researcher and a professor and I am very happy that we recently started to collaborate. Thank you for your discussion and insight on complex topics. I appreciate to be able to benefit from your scientific expertise and I am hopeful we will develop a great experiment together.

I would like to express my sincere appreciation to the members of the assessment committee for evaluating this thesis. Thank you Prof. Dr. Marc Vooijs, Prof. Dr. Mark Hill, Prof. Dr. Dirk De Ruysscher, Dr. Marijke De Saint-Hubert and Dr. Matthias Bauwens.

I will try to follow a rather chronological order and consider that the journey to achieve my PhD started in Brazil, a while back, when I was pursuing my Physics degree. I would like to mention my great Professors from the dosimetry department Elisabeth Yoshimura and Emico Okuno who introduced me to radiation physics. Also my friends and fellow Physics colleagues who helped, encouraged and celebrated with me the ups and downs of my undergrads.

I need to mention and thank Luana for the opportunity she gave me in the Belgian Nuclear Research Centre (Mol, Belgium) and her friendship ever since, I hope we can collaborate again. In Mol, I happily established great network of friends, which have been supporting and celebrating together for the last eight years. A special thanks to Clarita, and to Dario, Francesco, Matteo, Julie, Luca, Delphine, Angela, Jérémie, it has been great to have you all on this side of the world.

I would like to mention my late supervisor from NUI Galway, Wil van der Putten, a great and kind Dutch man in Ireland, who originally invited me to follow a PhD. He had an extensive knowledge of the Medical Physics field, having established the clinical department and the MSc course. The limited time we shared during my $\mathrm{PhD}$ was always positive and he is certainly missed by everyone who got to know him. Margaret Moore continued my supervision before my transfer to the Netherlands. She is an admirably strong woman, extremely capable, whom I can only be thankful to have met. I also appreciate the effort and time spent by everyone in the department of medical physics and bioengineering in Galway who taught, trained and considered me as an integrant of the group, especially Gordon, Aiofe and Sinead. 
Living in Ireland was a great side of my $\mathrm{PhD}$. I learned a lot from the Irish and always felt welcome. It is a marvellous country, where one learns to cherish the (rare) good weather and the (always) good pub atmosphere. I am thankful to have met wonderful people who also supported my $\mathrm{PhD}$ experience in Ireland. Bárbara, Carlos, Eoin and Kathy at home as well as Ali, Eoin, Letty, Kirsten, Laura, Deidre, Nina, Niahm, Salam, Alba, Mike at NUI Galway thank you for the great experience.

As moving to the Netherlands and re-starting half-way through my $\mathrm{PhD}$ required a lot of adaptation and long working hours, without some people it would have been impossible. Isabel you were the best partner I could have found in this journey. You are the most determined person I have ever met, you manage to juggle all your different and as important tasks and only few people know, but you have an enormous heart. You helped me in so many ways, became a dear friend and I am sure that any path you pursue will be a successful one. Louise, you are one of the most caring people I have ever met and a great medical doctor. I appreciate your patience with all my questions and I really owe you for all the diagnosis throughout the years. Your arrival in Maastricht coincided with mine, we shared many of the same experiences and I really hope you can resume your career soon, I am sure the world is a better place with you as a doctor in it. Murillo, I always enjoy talking to you and seeing how selfless and generous you can be. It is never difficult for you to help and understand others even when life might be difficult on you. I also appreciate your many peculiar hobbies. Although I should add a citation here, Isabel, Louise, Gabriel, Murillo, Planck and Carlos-bacon, the best nights in Maastricht were spent in your company.

Brent it has been a pleasure to work and have long conversations with you. You make my days a lot funnier and enjoyable, thank you. Cecile, you have always been kind and helpful to me. Although you had to face a lot of different situations during your $\mathrm{PhD}$, I am sure you will do a great job and come out a lot stronger. Janita, it was great having met you in Ireland, coincidently in Maastricht and we'll hopefully meet again in the future. Lotte, I appreciate all the guidance you've given me when I started. During my years in Maastro I met many great and interesting people in the research environment, which have contributed to a great experience, thank you Mark, José, Shane, Timo, Frank, Stefan, Behzad, Aniek, Evelyn, 
Relinde, Jurgen, Lucas, Matilde, Ruben, Blake, Henry, Simon, Turkey, Karen, Rianne, Ala, Natasja, Rob, Rosa, Teun, Sebastian, Yvonka, Abdalla, Alberto, Anshu, Leonard, Johan, Hajar, Ananya, Petros, Rianne, Biche, Ibrahim, Ivan, Matthijs, Giacomo.

I would like to thank Wouter for giving me an opportunity in the clinic with the (fancy) title of Innovation Physicist as well as my colleagues Femke, Colien and Vicki for all your kindness and support during the end of my $\mathrm{PhD}$.

Sonia, grazie per il supporto che mi hai dato, per la tua positività e per permettermi di far pratica con il mio italiano improvvisato. Allo stesso modo, Gloria e Ilaria, è stato bello chiaccherare anche brevemente con voi: siete delle brillanti scienziate, sempre pronte a darmi una mano. Spero di poter condividere con voi in futuro qualche momento in più fuori dalla clinica.

Continuando in italiano: Irma e Carlo, grazie per il vostro aiuto, per essere stati comprensivi e disponibili negli ultimi anni; mi fate sentire sempre a casa in Italia. Un grazie speciale a Irma per esser qui presente alla mia difesa. Matteo e Morena, grazie per avermi aiutato quando mi sono trasferita in Olanda: senza di voi, non penso sarei riuscita a stare qui. Grazie anche per avermi accettata come amica, anche se capire il mio italiano non è sempre facile.

Petra and Vivi, vocês são as minhas grandes companheiras de todos os momentos. Quem eu sou e minhas experências estão intrinsicamente conectados ao que somos juntas. Nós crescemos, aprendemos e nos permitimos juntas. Cassia, querida, é tão gostoso ser sua amiga. Nem parece mas já faz tantos anos conhecemos, apoiamos e nos confidenciamos. Mario você é um grande amigo de todos os momentos desde sempre, obrigada por todo apoio. Vocês acompanharam esse processo todo comigo, torceram, enxugaram lágrimas e, se agora eu recebo um título, ele é um pedaço de vocês. Obrigada.

Vó, tias e tio, muito obrigada por todo apoio ao longo desses anos distante, vocês me dão força para seguir em frente. 
Laura, você é a pessoa que ama com mais intensidade que eu conheço. Você é determinada, incansável, obstinada, meticulosa, engraçada, inteligente, você é para sempre o meu nenê. Eu tenho tanto orgulho de você. A distância sempre dói quando eu penso em você. Nós crescemos na mesma casa, vivemos muitas das mesmas experiências e apesar de agirmos muitas vezes de modos diferentes, somos essencialmente iguais. Te ter como irmã me impulsiona. Eu sou feliz e grata que o seu trabalho agora esta entrelaçado ao meu neste livro.

Mãe, é preciso mais que um livro para te agradecer. Você é a força que gera movimento. Você move minha vida, a da minha irmã, da Abigail e de toda a nossa família. Você derrotou gigantes e moinhos para nos dar a vida que temos, sempre nos colocando a frente. Você me deu educação e, se eu consigo enxergar o mundo de forma crítica, se eu consigo hoje defender um doutorado é porque você me impulsionou. Você é a pessoa mais forte que eu conheço e ao mesmo tempo a mais doce. Sempre que eu penso em ser mãe torço para que consiga ser um pedacinho do que você é. Muito obrigada, mãe.

Fabio, grazie. Thank for your patience and your help over the last years. Thank you for understanding and participating in this odyssey from the very beginning. For adapting to my life in all these different countries. For all the late nights and weekends trying to figure out this $\mathrm{PhD}$ together, this title is as much yours as it is mine. For all the assistance so my life would still function while I was working more hours than there are in a week. Thank you for being my practical half, for putting my dreams in motion. For being the second speaker of this dialect that only the two of us speak. Thank you for sharing your life with me. We achieved this together and there is so much yet to come.

And so it goes. 
Acknowledgements 



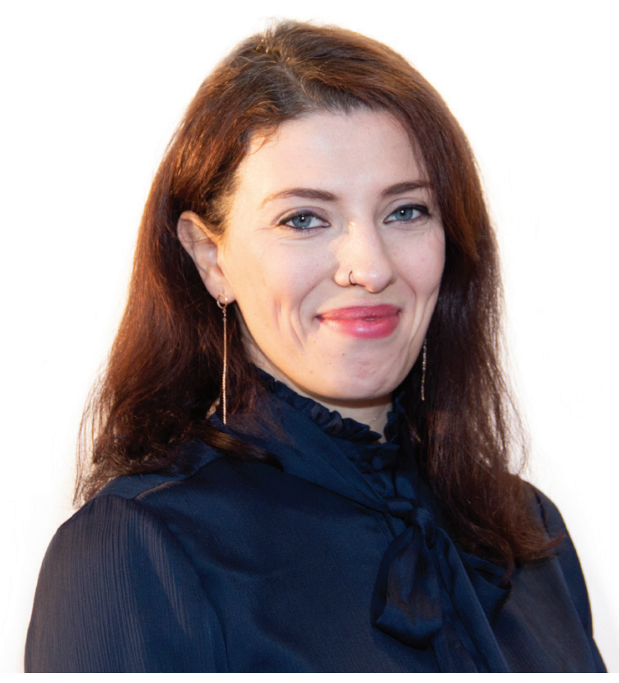

\section{Curriculum Vitae}

Ana Vaniqui was born on the 27th of June 1986 in Sao Paulo, Brazil. After a high school with a technical degree in Advertising, and in an attempt to satisfy her scientific curiosity, she went on to study Physics in the University of Sao Paulo (USP). During her undergraduate years, she worked in the private sector with Radiation Protection for medical and nuclear installations and with the licensing, commissioning and production of a pioneer cyclotron and radiopharmaceutical company. The latter work took her to the International Atomic Energy Agency (IAEA) in Vienna, where she presented and discussed the innovative aspects of the project. After graduation, she worked in different dosimetry projects at the Belgian Nuclear Research Centre $(\mathrm{SCK} \cdot \mathrm{CEN})$ related to the installation and characterization of an Am-Be neutron source for calibrations and the characterization of a new neutrons detector material for medical applications. In this period, she also worked at publishing houses editing and producing with Physics content. 
In 2014, shortly after she started a Master degree in Nuclear Engineering in Pisa, Italy, she was awarded a grant to pursue a PhD in Medical Physics. She started the PhD at the National University of Ireland, Galway (NUIG) and spent two years working on the field of adaptive and dose-guided clinical radiotherapy, learning the Medical Physicist's clinical routine and teaching at undergraduate and master's levels. In 2016, she left Ireland to join the research team of Prof. Dr. Frank Verhaegen at MAASTRO clinic in Maastricht, The Netherlands. During her PhD research, and as a part of Prof. Verhaegen's team, she investigated topics such as pre-clinical imaging (CT, dual-energy CT, CBCT, image reconstruction), treatment (external beam photons and protons), dosimetry (film, ion-chamber, EPID), Monte Carlo simulation of radiation and artificial intelligence techniques. In 2019, she joined the Physics Innovation team at Maastro Clinic, and currently works as an Innovation Physicist, connecting research and clinical practice. 



\section{Publication list}

A. Vaniqui, B.R. Walters, G.P. Fonseca and F. Verhaegen. Dose to water versus dose to medium from cavity theory applied to small animal irradiation with kilovolt x-rays. Phys Med Biol. 2019 Aug; 64(i6):I6500i.

A. Vaniqui, L.E.J.R. Schyns, I.P. Almeida, B. van der Heyden and F. Verhaegen. The effect of different image reconstruction techniques on preclinical dual-energy CT and image quality, Br J Radiol., 2019 Mar; 92(I095), 20180447 .

A. Vaniqui, B. van der Heyden, I.P Almeida, L.E.J.R. Schyns, S.J. van Hoof, and $\mathrm{F}$. Verhaegen. On the determination of planning target margins due to motion for mice lung tumours using a ${ }_{4} \mathrm{D}$ MOBY phantom. Br J Radiol., 2019 Mar; 92(I095), 20180445.

A. Vaniqui, L.E.J.R. Schyns, I.P. Almeida, B. van der Heyden, S. J. van Hoof, and F. Verhaegen, The impact of dual energy CT imaging on dose calculations for pre-clinical studies. Radiat Oncol. 2017 Nov; I2: I8I.

I.P. Almeida, A. Vaniqui, L.E.J.R. Schyns, B. van der Heyden, G. VilchesFreixas, J. Cooley, T. Zwart, A. Langenegger, F Verhaegen. Exploring the capabilities of a clinical proton beam with an adaptive aperture for preclinical research. Br J Radiol., 2019 Mar; 92(I095), 20180446.

B. van der Heyden, G. Fonseca, M. Podesta, I. Messner, N. Reisz, A. Vaniqui, P. Steininger, F. Verhaegen. Modelling of the focal spot intensity distribution and the off-focal spot radiation in kilovoltage X-ray tubes for imaging. Sumitted to Physics in Medicine and Biology (PMB-I09495), October, 20I9.

B. van der Heyden, M. Podesta, L.E.J.R. Schyns, A. Vaniqui, I.P. Almeida, S.J. van Hoof, D.B.P. Eekers and F. Verhaegen. Automatic multi-atlas based organ at risk contouring in mice. Br J Radiol., 2019 Mar; 92(I095):20180364. 
L.E.J.R. Schyns, B. van der Heyden, I.P. Almeida, A. Vaniqui, and F. Verhaegen. Murine versus human tissue compositions: implications of using human tissue compositions for dose calculations in mice. Br J Radiol., 2019 Mar; $92($ I095):20180454.

V.S. Iglesias, S.J. van Hoof, A. Vaniqui, L.E.J.R. Schyns, F. Verhaegen, J. Theys, L. Dubois and M. Vooijs, Non-invasive imaging and radiotherapy planning of a preclinical model of orthotopic NSCLC tumour to improve preclinical treatment testing platforms. Br J Radiol., 2019 Mar; 92(I095):20180476.

I.P. Almeida, L.E.J.R. Schyns, A. Vaniqui, B. van der Heyden, G. Dedes, A.F. Resch, F. Kamp, J.D. Zindler, K. Parodi, G. Landry and F. Verhaegen, Monte Carlo proton dose calculations using a radiotherapy specific dual-energy CT scanner for tissue segmentation and range assessment. Phys Med Biol. 2018 May; 63(II):I15008

B. van der Heyden, L.E.J.R. Schyns, M. Podesta, A. Vaniqui, I.P. Almeida, G. Landry and F. Verhaegen, VOXSI: a voxelized single- and dual-energy CT simulator for quantitative imaging. Phys and Imag Radiat Oncol. 20r8 Apr; 6:47-52.

L F Nascimento, E D'Agostino, A C S Vaniqui, C Saldarriaga, F Vanhavere, Y De Deene, Characterisation of OSL and OSLN droplets for dosimetry. Radiat Prot Dosimetry, 20I4 Oct; I6I(I-4). 


\section{Presentation \& Abstracts}

A. Vaniqui. A framework for plan quality assessment using prediction of organ-at-risk dose metrics. Scientific project day - RKF radiotherapy, Erasmus MC, Rotterdam, 2org November.

A. Vaniqui. A Plan QA framework: why and how. ESTRO Physics Workshop. Budapest, Hungary. 2019 October.

A Vaniqui, R. Canters, F. Vaassen, C. Hazelaar, I. Lubken, K. Kremer, C. Wolfs, W. van Elmpt. Plan quality assessment for rectal cancer patients using prediction of organ-at-risk dose metrics. Abstract submitted to ESTRO 2020, Vienna, Austria.

G. Vilches-Freixas, A. Vaniqui, F. Vaassen, C. Hazelaar, M. Öllers, S. Canisius, D. Tissen, E. van Enckevort, E. Rousch, K. Verhoeven, J. van Loon, D. De Ruysscher, G. Bosmans, W. van Elmpt, I. Rinaldi, S. Peeters Breath hold characteristics and intra-fraction motion during nasal high flow therapy assisted RT. Abstract submitted to ESTRO 2020, Vienna, Austria.

A. Vaniqui. Small animal imaging and therapy. Lecture for medical engineering students from the Technical University of Eindhoven. 2019 Feb/Sep; Maastricht, The Netherlands.

F. Verhaegen, A. Vaniqui, S. Van Hoof, I.P. Almeida, B. van der Heyden, P. Granton, J. Theys, M. Vooijs, L. Dubois. SP-ooo7 Technology for precision small animal radiotherapy research: Optimal use and challenges. Radiother Oncol., 2019 Apr; 133 $\left(\mathrm{S}_{3}-\mathrm{S}_{4}\right)$.

A. Vaniqui, B. van der Heyden. Small animal treatment planning workshop. 20I8 Dec; irth International Conference on Cachexia, Sarcopenia \& Muscle Wasting Maastricht, The Netherlands. 
F. Verhaegen, S. Van Hoof, L. Schyns, S. Yahyanejad, B. van der Heyden, A. Vaniqui, P. Granton, L. Dubois, M. Vooijs. SP-o223: State of the art and future developments of small animal imaging and radiotherapy platforms. Radiother Oncol., 20I8 Apr; I27(Sirg).

A. Vaniqui. Small animal imaging and therapy. Course for medical engineering students from the Technical University of Eindhoven. 2018 Feb/Oct; Maastricht, The Netherlands.

A. Vaniqui. Using a mathematical phantom to determine mice lung tumours planning target margins due to breathing. 20I8 May; Maastricht, The Netherlands.

A. Vaniqui, B. van der Heyden, I.P Almeida, L.E.J.R. Schyns, S.J. van Hoof, and F. Verhaegen. On the determination of planning target margins due to motion for mice lung tumours using a ${ }_{4} \mathrm{D}$ MOBY phantom. 2018 Mar; 4th Conference on Small Animal Precision Image-Guided Radiotherapy, Lisbon, Portugal.

L.E.J.R. Schyns, B. van der Heyden, I.P. Almeida, A. Vaniqui, D.B.P. Eekers and F. Verhaegen. Murine versus human tissue compositions: implications of using human tissue compositions for dose calculations in mice. 20I8 Mar; 4th Conference on Small Animal Precision Image-Guided Radiotherapy, Lisbon, Portugal.

I.P. Almeida, A. Vaniqui, L.E.J.R. Schyns, B. van der Heyden, G. VilchesFreixas, J. Cooley, T. Zwart, A. Langenegger, F. Verhaegen. Exploring the capabilities of a clinical proton beam with an adaptive aperture for preclinical research. 20I8 Mar; 4th Conference on Small Animal Precision Image-Guided Radiotherapy, Lisbon, Portugal.

S.J. van Hoof, A. Vaniqui, L.E.J.R. Schyns, B. van der Heyden and F. Verhaegen. Dose painting by combined couch motion and irradiation on an image guided small animal radiotherapy platform. 20I8 Mar; 4th Conference on Small Animal Precision Image-Guided Radiotherapy, Lisbon, Portugal. 
B. Yalvac, A. Vaniqui, E. Sterpin and B. Reniers. Response of alanine dosimeters to low energy X-rays and small fields. 2018 Mar; 4th Conference on Small Animal Precision Image-Guided Radiotherapy, Lisbon, Portugal.

A. Vaniqui, L.E.J.R. Schyns. Workshop on treatment planning for Maastricht University medical students. 2018 Jan; Maastricht, The Netherlands.

A. Vaniqui. Small animal imaging and therapy. Course for medical engineering students from the Technical University of Eindhoven. 2017 Oct; Maastricht, The Netherlands.

F. Verhaegen, I.P. Almeida, B. van der Heyden, L.E.J.R. Schyns, A. Vaniqui, M. Bellezzo, S.J. van Hoof, P. Granton, G. Landry, G. Fonseca, M. Podesta. The use of imaging information in Monte Carlo simulations. 20I7 Oct; International Conference on Monte Carlo Techniques for Medical Applications (MCMA2oi7), Naples, Italy.

A. Vaniqui. Computed tomography and X-ray diagnostics. 20II Aug; Course for the medical residency on radiation oncology of the Federal University of Sao Paulo.

A. Vaniqui, A.C.F. Sobreira, H. Nogueira, F.P. Jesus. Educational applications of particle accelerators. 2009; International Topical Meeting on Nuclear Research Applications and Utilization of Accelerators, International Atomic Energy Agency (IAEA), Vienna, Austria.

A. Vaniqui, A.C.F. Sobreira. Radioactive Material Transport. 2009; Dangerous goods meeting of COMCARGA (ANAC, INFRAERO, Anvisa, Ibama - national regulatory authorities of aviation, health and environment - and airline companies) Rio de Janeiro, Brazil.

A. Vaniqui, Radiological protection presentation and training at Port of Santos in order to change the ordinance which prevented the passage of Dangerous Materials through this harbour. Port of Santos, SP, Brazil, 2009. 


\section{Grant and Prize}

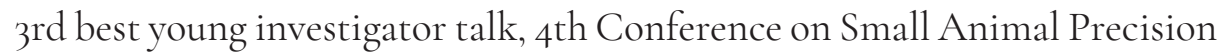
Image-Guided Radiotherapy. 2018 Mar; Lisbon, Portugal.

PhD grant: CAPES Scholarship and Science without Borders program from Brazil (BEX I2030/13-9), 20I4, Brazil. 

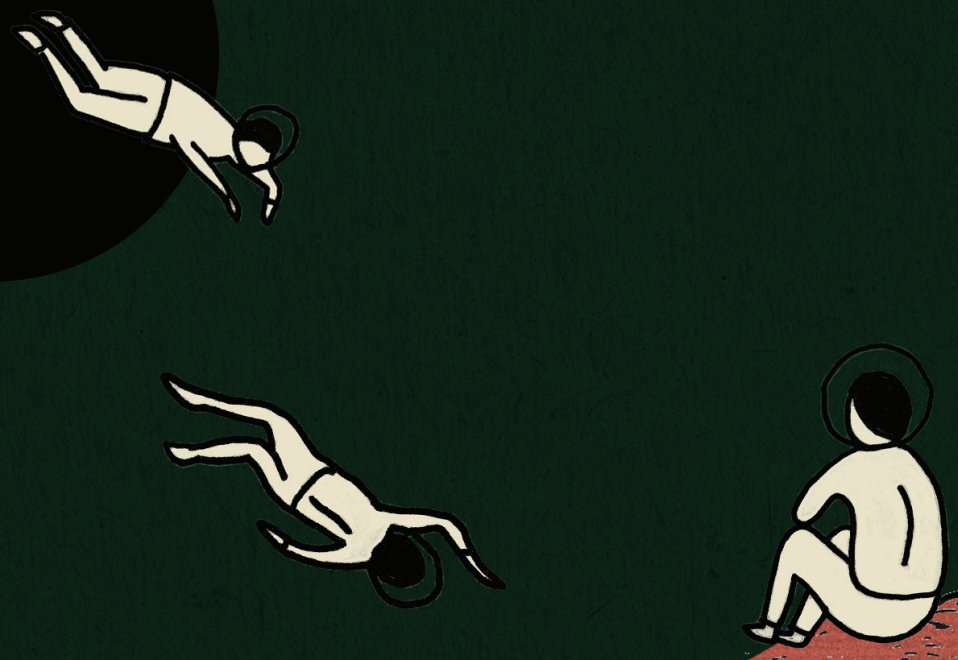

80.

ith

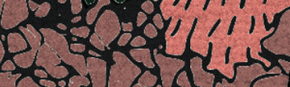
in sरो क्षे? पiर से दि? $\Omega$ miा if
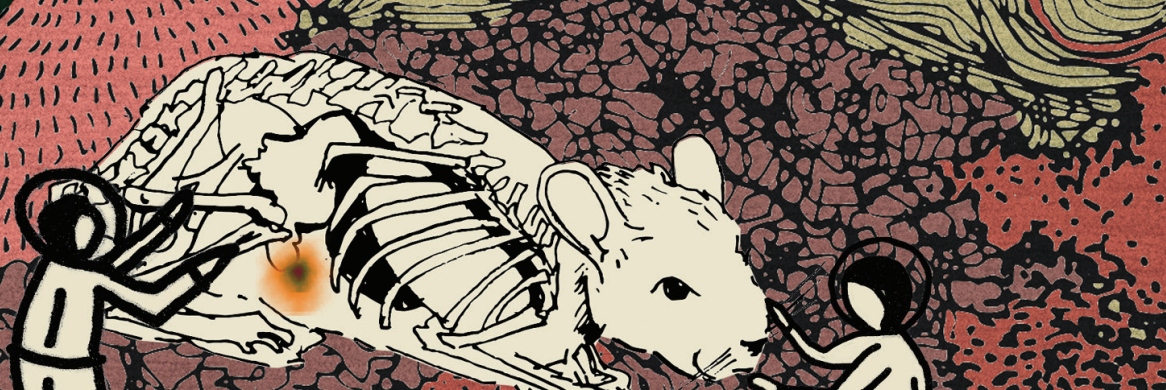